\title{
Perturbation of Pattern Formation in Dictyostelium Discoideum via Flow and Spatial Heterogeneities
}

\author{
Dissertation \\ for the award of the degree \\ "Doctor rerum naturalium" \\ at the Georg-August-Universität Göttingen \\ within the doctoral program \\ "Physics of Biological and Complex Systems" \\ of the Göttingen Graduate Center of Neurosciences, \\ Biophysics, and Molecular Biosciences (GGNB) \\ of the Georg-August University School of Science (GAUSS) \\ submitted by \\ Torsten Frank Eckstein \\ from Hamburg \\ Göttingen, 2020
}


Thesis Advisory Committee

\section{Dr. Azam Gholami}

Laboratory for Fluid Physics, Pattern Formation and Biocomplexity

Max Planck Institute for Dynamics and Self-Organization

Prof. Dr. Stefan Klumpp

Institut für Dynamik komplexer Systeme

Georg-August-Universität Göttingen

Prof. Dr. Jörg Enderlein

Drittes Physikalisches Institut

Georg-August-Universität Göttingen

Members of the examination board

\section{Referee:}

Dr. Azam Gholami

Laboratory for Fluid Physics, Pattern Formation and Biocomplexity

Max Planck Institute for Dynamics and Self-Organization

\section{Co-referee:}

Prof. Dr. Jörg Enderlein

Drittes Physikalisches Institut

Georg-August-Universität Göttingen

Further members of the examination board:

\section{Prof. Dr. Stefan Klumpp}

Institut für Dynamik komplexer Systeme

Georg-August-Universität Göttingen

\section{Prof. Dr. Jörg Großhans}

Deptartment Developmental Biochemistry

University Medical Center Göttingen

\section{Dr. Michael Wilczek}

Max-Planck-Research Group - Turbulence, Complex Flows \& Active Matter

Max Planck Institute for Dynamics and Self-Organization

\section{Dr. David Zwicker}

Max-Planck-Research Group - Theory of Biological Fluids

Max Planck Institute for Dynamics and Self-Organization

Date of the oral examination: 26.03.2020 


\section{Abstract}

In this thesis, we investigate the signaling process of the social amoeba Dictyostelium discoideum (D. discoideum) as a model reaction-diffusion-advection system. Colonies of these cells are an excitable medium that exhibits waves of the chemoattractant cAMP. The cells produce spiral waves and target patterns, which they use to aggregate. In the natural habitat of $D$. discoideum, the soil, the amoebae are exposed to perturbations by fluid flows induced by rain and by obstacles in the constraint of their environment. Here, we perturb pattern formation by means of advective flows and spatial heterogeneities, in three different studies. The experimental findings are compared to theoretical results, in order to probe the limits of the theory.

In the first part, we investigate pattern formation in $D$. discoideum under the influence of advecting flows. To generate constant highly controlled flows of magnitude comparable to the flows the cells experience in nature due to rain, we utilize microfluidics. We find that the wave speed depends on the applied flow speed, while the wave period is constant at different speeds. Together with observations of cell aggregation even at high flow speeds, this indicates that pattern formation in D. discoideum is quite robust to this type of perturbation. Further, we find a scaling of the wave width with the applied flow speed and significant changes in the wave shape. It changes from planar waves to parabolic waves with increasing curvature at higher flow speeds. At high speeds the waves are only initiated at the upstream boundary of the channel. In addition, their specific initiation point in the lateral dimension at this boundary impacts the wave shape. Comparing experimental results to theoretical work, we confirm that waves can be initiated in the system by creating a heterogeneous initial state of the simulated cells and using a developmental path model to simulate how the cells develop during the experiment. Moreover, we observe that a common simplification of the model cannot reproduce the correct wave shape at high flow rates. In this simplified model, the transport of cAMP from within the cells to the extracellular medium is assumed to be instantaneous. Therefore it fails to include the slow dynamics of cAMP release from the cells that we find to be crucial for the wave shape at high flow velocities.

Inspired by simulations that predict wave creation induced by flow and a Dirichlet boundary condition at low cAMP degradation, we study pattern formation under flow for $\mathrm{pdsA}^{-}$cells. These cells lack the cAMP degradation agent PDE1, and consequently can neither form cAMP waves nor aggregate. In agreement with the prediction, we find that flow can act as an effective degradation, allowing the cells to produce waves. These experiments give us insight into the time scale of the cells' development, as no patterns are observed before three hours of constant flow. Interestingly, we observe waves whose amplitude decays while traveling along the channel. The length over which these waves decay scales with imposed flow speed. Moreover, the response is divided into an early and a late regime with considerable difference in dynamics. Finally, we find spontaneous pattern formation of these degradation deficient cells, if supplied with flow for at least 3.5 hours. This suggests that, if the cells are supplied with flow for at least 3 hours, they start producing a homologue of PDE1, possibly PDE4 or PDE7. In connection to theoretical studies we confirm experimentally that a Dirichlet boundary condition can 
destabilize an otherwise stable reaction-diffusion-advection system.

Finally, we study the influence of spatial inhomogeneities on pattern formation in $D$. discoideum, using a two-dimensional periodic distribution of non-excitable obstacles. We find that there is no strong interaction between obstacles and spiral tips, unless one is initiated very close to an obstacle by chance, resulting in pinning. However, if we initially starve the cells and add caffeine to reduce the excitation threshold of the cells, the dynamics are altered drastically. The cells then show target waves centered on the obstacles, which dominate the system dynamics in the long term. This results in highly regular aggregation territories, reflecting the geometry of the obstacles. Therefore, we found a way to control pattern formation in this system.

Keywords: Biophysics, Dictyostelium discoideum, Pattern formation, Reaction-diffusionadvection 


\section{Contents}

$\begin{array}{ll}\text { Nomenclature } & 1\end{array}$

1 Introduction $\quad 3$

1.1 Motivation . . . . . . . . . . . . . . . . . . 3

1.2 Dictyostelium discoideum . . . . . . . . . . . . . . . 4

1.2.1 D. discoideum life cycle . . . . . . . . . . . . . 5

1.2.2 Pattern formation in D. discoideum . . . . . . . . . . . . . . . . . 9

1.2 .3 Wave characteristics . . . . . . . . . . . . . . 15

1.2 .4 Notable strains . . . . . . . . . . . . . . . . . . . . . 19

1.3 Reaction-Diffusion systems . . . . . . . . . . . . . . . . . . 21

1.4 Excitable Media . . . . . . . . . . . . . . . . . . . . . 26

1.5 Modeling patterns in D. discoideum . . . . . . . . . . . . . . 30

1.5.1 Martiel-Goldbeter model . . . . . . . . . . . . . . . . . 30

1.5.2 Wave creation in D. discoideum models . . . . . . . . . . . . 33

1.5.3 Other models for pattern formation in D. discoideum . . . . . . . 34

1.6 Methods . . . . . . . . . . . . . . . . . . . . 37

1.6 .1 Cell culture . . . . . . . . . . . . . . . . . . . . . . . . . . 37

1.6.2 Experimental setup . . . . . . . . . . . . . . . . . . . . . . . . . . . . . . . 38

1.6.3 Image processing . . . . . . . . . . . . . . . . . . . . . 41

1.6.4 Image analysis . . . . . . . . . . . . . . . . . . . . . . 47

2 Influence of fast advective flows on pattern formation of Dictyostelium discoideum

3 Experimental observation of boundary-driven oscillations in a reactiondiffusion-advection system

4 Spatial heterogeneities shape collective behavior of signaling amoeboid cells 89

5 Discussion $\quad \mathbf{1 2 5}$

5.1 Pattern formation of $D$. discoideum under the influence of an advective flow 125

5.1 .1 Fast advective flows . . . . . . . . . . . . . . . . 125

5.1.2 Boundary-driven waves in pdsA ${ }^{-}$cells . . . . . . . . . . . . . 127

5.2 Pattern formation of $D$. discoideum under the influence of non-excitable obstacles . . . . . . . . . . . . . . . . . . 130

6 Conclusions and Outlook 133 
Contents

Acknowledgments

153

Curriculum Vitae

155 


\section{Nomenclature}

\begin{tabular}{|c|c|}
\hline Acronym & Definition \\
\hline D. discoideum & Dictyostelium discoideum \\
\hline cAMP & 3',5'-cyclic Adenosine Monophosphate \\
\hline cGMP & cyclic Guanosine Monophosphate \\
\hline $\mathrm{cAR}$ & cyclic Adenosine Monophosphate Receptor \\
\hline G protein & Guanine Nucleotide binding Protein \\
\hline Ras & Rat Sarcoma \\
\hline Rap1 & Ras-related Protein 1 \\
\hline MAP kinase & Mitogen-activated Protein Kinase \\
\hline PI3K & Phosphatidylinositol 3-Kinase \\
\hline PKB & Protein Kinase B \\
\hline CRAC & Cytosolic Regulator of Adenylate Cyclase \\
\hline TORC2 & Target of Rapamycin complex 2 \\
\hline PDE & Phosphodiesterase \\
\hline $\mathrm{ACA}$ & Adenylate Cyclase of Aggregation stage \\
\hline $\mathrm{ACG}$ & Adenylate Cyclase of Germination stage \\
\hline $\mathrm{ACB}$ & Adenylate Cyclase B \\
\hline ATP & adenosine triphosphate \\
\hline $\mathrm{PDE}$ & phosphodiesterase \\
\hline pdsA & phosphodiesterase $\mathrm{A}$ \\
\hline PDI & phosphodiesterase inhibitor \\
\hline BZ reaction & Belousov-Zhabotinsky reaction \\
\hline MG-model & Martiel-Goldbeter model \\
\hline $\mathrm{Pb}$ & phosphate buffer \\
\hline rpm & rotations per minute \\
\hline FFT & fast Fourier transform \\
\hline PDMS & Polydimethylsiloxane \\
\hline PMMA & Polymethyl methacrylate \\
\hline WT cells & wildtype cells \\
\hline
\end{tabular}





\section{Introduction}

\subsection{Motivation}

Pattern formation is a ubiquitous concept in nature, explaining how complex spatiotemporal patterns can arise from an initially homogeneous, non-equilibrium system [1]. In this non-linear process, regular patterns develop without the requirement of excessive locally stored information and in a self-organized manner. Pattern formation is invoked to study a diverse set of problems in nature, both for living and nonliving systems. Examples include dune formation [2], the formation of salt playa in the desert [3] and roll formation in a shear flow, called Taylor-Couette flow [4]. For living systems, pattern formation is mainly concerned with the large-scale organization of cells. A general feature of pattern forming systems is that the patterns define directions in an initially isotropic space. This property is used by cells in morphogenesis, where spatial information is crucial for their fate. The study of pattern formation in the context of morphogenesis dates from 1952 when Turing suggested that interacting chemical species could be the basis for cell differentiation [5]. Examples of the involvement of pattern formation in morphogenesis are quite common, with the stripe patterns of zebra fish being a canonical example [6]. However, more complicated organisms have been shown to use pattern formation to determine the spacing of regular features, such as feather buds in chicks, hair follicles in mice and lymphangiogenesis in zebra fish embryos [7-9]. Various other living systems are studied in the context of pattern formation as well, such as the growth of bacterial colonies [10].

Reaction-diffusion systems are widespread in nature, both in animate and inanimate structures. In these systems, multiple chemicals react and diffuse in an aqueous environment, leading to patterns in chemical concentration. Examples of inanimate systems include CO oxidation waves on a platinum surface and the Belousov-Zhabotinsky reaction $[11,12]$. In animate systems, reaction-diffusion dynamics allow for robust signal relay and self-organization of cells over macroscopic distances to define cell and gene expression. The most prevalent patterns in biological systems are the ones animals show to the outside world such as the patterns on mollusk shells [13]. However, there are also reaction-diffusion systems in the human body, whose failure leads to illnesses, as the electrical waves in cortical tissue and the heart muscles [14,15]. Another example for a reaction-diffusion system are cAMP waves during the aggregation of Dictyostelium discoideum (D. discoideum) amoebae, which the cells use for communication over macroscopic distances [16]. Based on the governing equations of reaction-diffusion systems, intrinsically different biological phenomenona can be described similarly. Therefore, it is possible to draw conclusions on multiple systems based on the study of a specific one. 
This motivated our study of cAMP waves during the aggregation of $D$. discoideum cells. The social amoeba is a biological model system for pattern formation, showing reaction-diffusion dynamics on the multicellular level. Central advantages of these cells are their widespread usage in research and their ease of manipulation in a laboratory frame, especially on the genetic level. This allows for the deletion of single genes and the corresponding proteins from the pattern forming system, making the role of the proteins accessible. In such studies it is possible to verify models and their assumptions in a very direct way. We conducted experiments on the pattern formation of $D$. discoideum cells, comparing and relating our findings back to theory and simulations. Our approach is both inspired by the theoretical description of this excitable medium and by the natural environment the cells live in. Our aim is to test the limits of theory by conducting specific experiments and comparing them to simulations of the system. Using this methodology, we hope to advance both the study of this specific system and the general understanding of pattern formation, specifically of reaction-diffusion systems. Since this type of system is quite common in nature, results should be applicable for a diverse range of problems. This includes medical applications connected to malformations based on dysfunctional cell expression patterns.

\subsection{Dictyostelium discoideum}

Dictyostelium discoideum (D. discoideum) amoebae show large scale pattern formation based on reaction-diffusion dynamics, which they utilize to aggregate. As of today, D. discoideum is considered a model organism for the study of chemotaxis, signal relay and pattern formation [17, p. 72ff.]. The first species in the Dictyostelium genus, D. mucoroides, was described in 1869 by Oscar Brefeld [18]. Brefeld's studies laid the foundation for the field of Dictyostelium research, paving the way to the study of Dictyostelium cells and their different components. While he could not determine what exactly the amoebae were feeding on, Brefeld performed extensive studies and observed many of the defining features of the Dictyostelium genus. He noted that the cells aggregated and formed a stalk complete with fruiting body. Moreover, he mentioned spore capsules and their ability of germination even after several weeks of storage.

The Dictyostelium species studied in this thesis, Dictyostelium discoideum, was described by Raper in 1935 [19], who isolated the species from decaying leaves found in North Carolina. Raper examined the formation of an intermediate aggregate form, an elongated tubular shape that can migrate and is known today as the "slug" form. Raper went on to study $D$. discoideum and made many important discoveries, among which the observation that $D$. discoideum would grow if incubated with a great number of different bacteria [20] (32 species were tested in his study). This meant that, in contrast to other Dictyostelium species, D. discoideum could be grown at relative ease, which would in time make it the most studied Dictyostelium species.

The $D$. discoideum cell line we are using is a species from a genus of amoebae commonly referred to as "slime molds" or "social amoebae". The first of these names, "slime molds", is somewhat of a misnomer as the cells are not fungi, although they produce 
fruiting bodies complete with spores. In contrast, the second name "social amoebae" describes a key feature of the Dictyostelium genus, namely the cells' ability to act cooperatively to ensure survival. Cooperation in $D$. discoideum occurs as a response to adverse environmental conditions. As long as D. discoideum cells find enough food (bacteria) in their environment, they will stick to a solitary lifestyle in the soil. However, when nutrients are depleted, the cells start developing and sending chemical signals to each other. This leads to the formation of streaming territories in which the cells aggregate into clusters of up to $10^{6}$ cells [21], see Fig. 1.1. The cell aggregates can then develop further, forming multicellular structures ultimately resulting in fruiting bodies. These contain spores that enable the species to survive adverse conditions.

D. discoideum cells use pattern formation to determine aggregation territories. The cell colonies act as a reaction-diffusion system, where the cells produce a signaling chemical called 3',5'-cyclic adenosine monophosphate (cAMP). The amoebae produce pulses of cAMP, which propagate through the aqueous environment as waves. Upon sensing an increased cAMP concentration, neighboring cells start producing the chemical as well, thus relaying the wave. Furthermore, the cells perform chemotaxis in response to cAMP, meaning that they move in the direction of positive gradients of the chemical. Because of the cell movement in response to the cAMP waves, the cells' shape changes. Considering that cell shape is connected to the cells' ability to scatter light, this means that cAMP waves can be observed indirectly by using dark-field microscopy. In this method, the main light beam is adjusted in such a way that unscattered light is not collected. This allows for highlighting changes in light scattering of the sample, see Fig. 1.2 for an example of a dark-field experiment.

\subsubsection{D. discoideum life cycle}

To provide a broader context to the pattern formation in $D$. discoideum, a more detailed description of the life cycle of cells is necessary. Refer to Fig. 1.1 for a sketch, showing different stages in the life cycle. The pattern formation and aggregation process is shown in Fig. 1.2.

As long as D. discoideum cells find adequate amounts of food in their environment, the cells are in the "feeding" or "trophic" phase of their life cycle, in which the amoebae reproduce asexually by cell division. Whenever nutrients are scarce, the cells respond rapidly, exiting the cell cycle and ceasing other activities that are connected to growth [21]. Instead, genes necessary for aggregation activate and the cells start producing proteins to enable signaling and signal responses in order to coordinate aggregation. D. discoideum cells can sense impending starvation via a secreted molecule called pre starvation factor [23], which leads to the upregulation of genes necessary for aggregation. The amoebae can sense their local density in a similar manner via excreted molecules called counting factor [24]. The stark difference in cell behavior means that there is a strict division between growth phase and starvation-induced response phase. There are two other responses to starvation as well, the formation of microcysts and of macrocysts [21]. In the first case, the cells enter a dormant state in a solitary fashion [21]. In the latter case, the cells perform a sexual cycle with two cells of different mating types fusing 


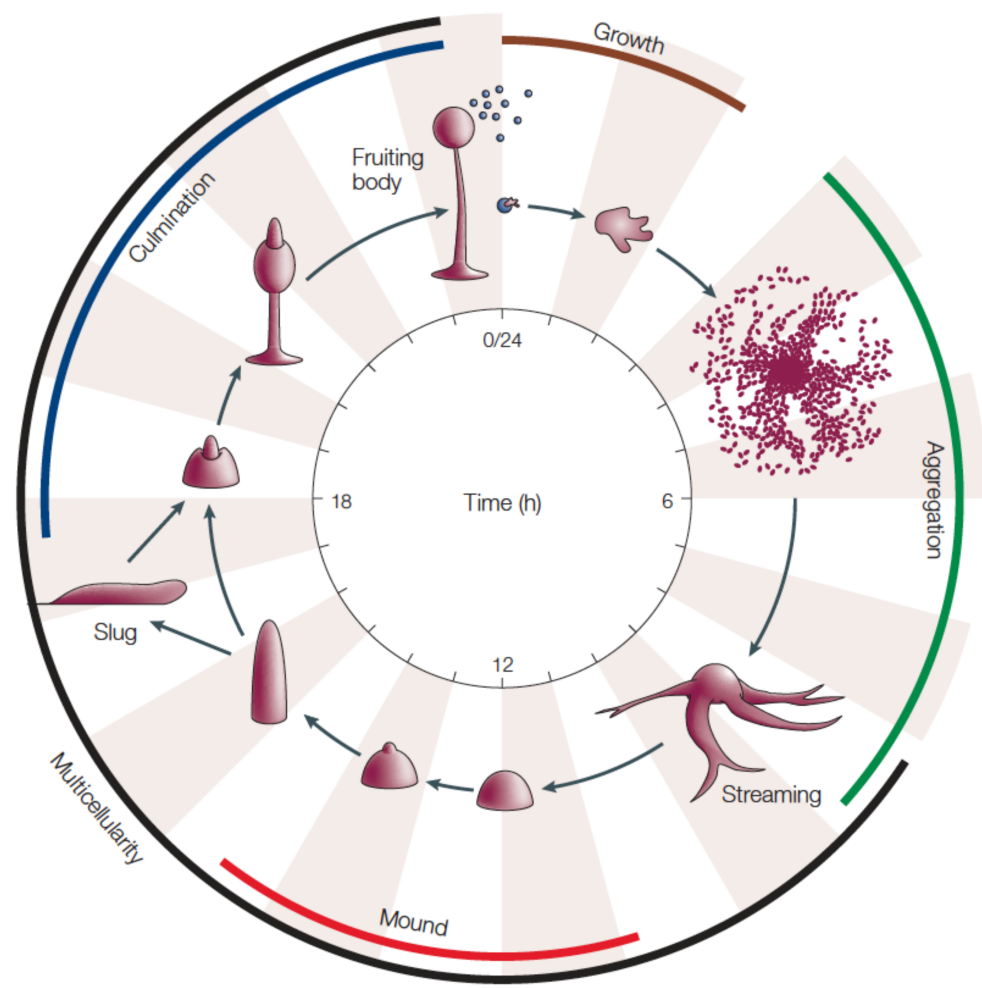

Figure 1.1: Sketch showing the life cycle of D. discoideum cells. Under growth conditions the amoebae grow and live a solitary lifestyle. Under starvation conditions, the cells aggregate, forming streams and producing a cell mound. This mound elongates and can first transform into a moving pseudoplasmodium or directly move on to the culmination stage. In this phase, the cells form a fruiting body suspended from a stalk of dead cells. The spores in the fruiting body are prepared for long range dispersal and will germinate, given suitable conditions. Reprinted by permission from Springer Nature Customer Service Centre GmbH: Nature nature reviews molecular cell biology, Insights into morphogenesis from a simple developmental system, Chisholm, Rex L. and Firtel, Richard A., Copyright 2020 (2004). [22]

and producing offspring [21].

The mechanism for signal generation and relay in $D$. discoideum is based on a chemical called 3',5'-cyclic adenosine monophosphate (cAMP) and on the cells' response to it [25]. The amoebae produce pulses of cAMP, which propagate through the aqueous environment as waves. Upon sensing increased cAMP concentration, neighboring cells start producing the chemical as well, thus relaying the wave. Furthermore, the cells respond chemotactically to cAMP, meaning that they move towards increased amounts of the chemical. On a macroscopic level, these waves form spiral or target patterns [26], see Fig. 1.2a). Both of these wave types are spatially extended pulses that travel through the system. A determining feature of spiral waves is that they end in a point, which rotates around an area called the spiral core. In contrast, a target center consists of 


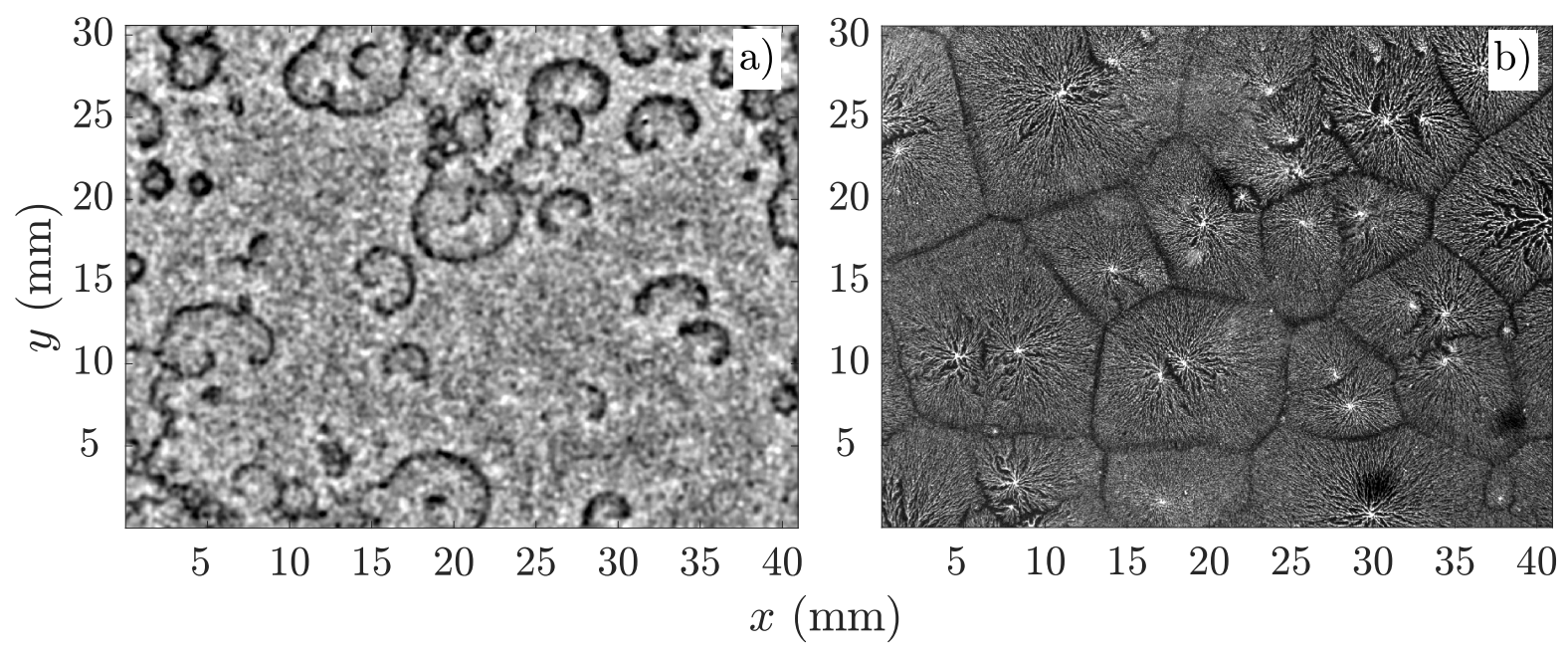

Figure 1.2: Pattern formation and aggregation of D. discoideum in an experiment with cells plated on $1.5 \%$ phosphate buffer agar. In a) the pattern formation is shown in a normalized and band-pass filtered image at 6 hours after the start of starvation. b) shows aggregation streams after about 10 hours of experiment. Cells initially starved in shaking culture for 4 hours. Refer to chapter 1.6.3 for a description of the image processing techniques.

a number of concentric waves centered on a point from which they are induced. The cells form branching streams, in which the amoebae move closely packed towards the centers of these patterns, as shown in Fig. 1.2b). In this way, D. discoideum determines aggregation territories via pattern formation.
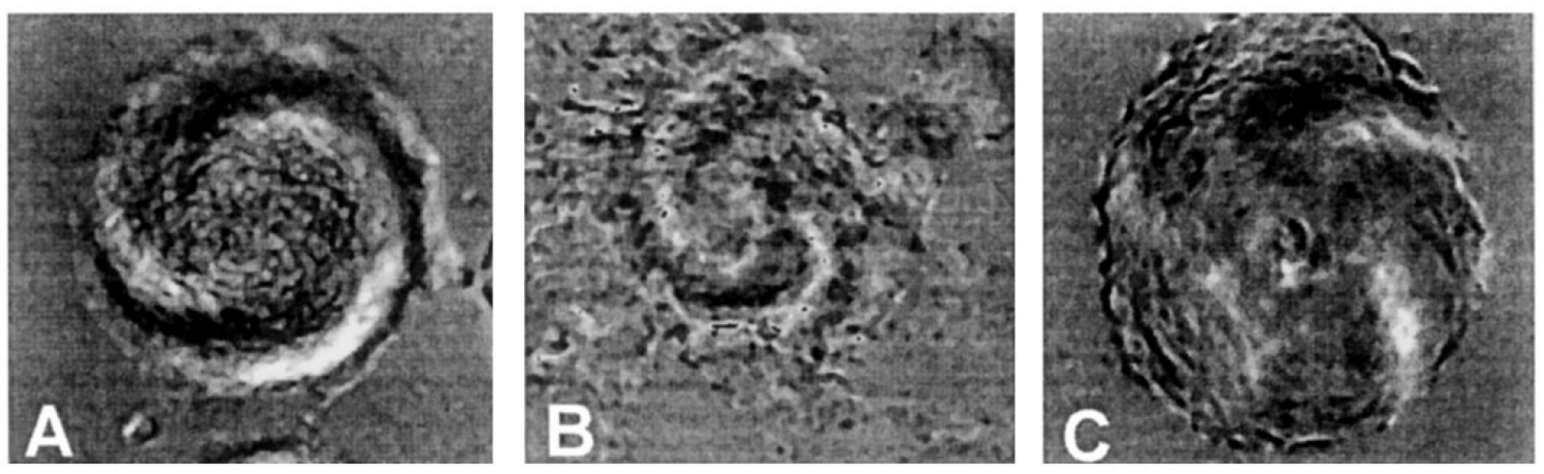

Figure 1.3: Dark-field images showing spiral waves in the mound stage of $D$. discoideum aggregation. The images show spirals with A) two arms, B) three arms and C) five arms. Reprinted figure with permission from Bakthier Vasiev, Florian Siegert, and Cornelis Weijer, Physical Review Letters, 78, 2489, 1997. Copyright 2020 by the American Physical Society. [27]

During the first 4 to 6 hours of aggregation, the cells will revert to the growth phase if supplied with nutrients. After this time, the amoebae lose their ability for phagocytosis, 
which makes them unable to revert and can be seen as a commitment to the developmental phase [28]. After streaming has been completed, D. discoideum forms an aggregate, also called mound, which is protected by a slime sheath containing cellulose [29], see Fig. 1.1. In this stage, the cells divide into precursor cells for the later spore and stalk cells [30]. Additionally, an organizing center is formed in the mound and spiral wave formation focused on this point can be observed [31], see Fig. 1.3.
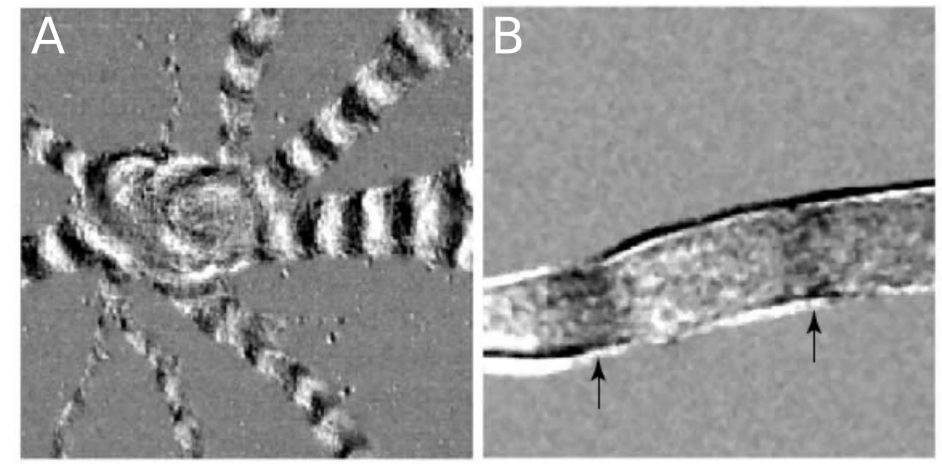

Figure 1.4: Images showing waves in D. discoideum aggregation. A) shows waves during the streaming phase. In B) planar waves in the slug stage are shown. Reprinted from Current Opinion in Genetics \& Development, 14, Cornelis J Weijer, Dictyostelium morphogenesis, 392-398, Copyright 2020 (2004), with permission from Elsevier. [32]

After further starvation, the cell mound starts to elongate, as shown in Fig. 1.1. From this stage the aggregate can enter into the culmination phase to produce a fruiting body or form a moving pseudoplasmodium (or "slug"), depending on environmental conditions such as light direction [33]. In the latter case, the aggregate forms an organizing center in the tip of the slug, made from prestalk cells [34]. The slug responds to optical signals and heat [33], moving to escape detrimental conditions. In addition, slugs disperse to ensure low local population density, based on the secretion of repellent molecules [35]. Pattern formation persists in the slug phase. During this time, the amoebae organize movement using cAMP scroll waves, the three-dimensional equivalent to spiral waves. These are centered on the organizing tip and transition to planar waves in the main slug body, see Fig. 1.4B) [36].

Once the slug has stopped moving, the aggregate enters the culmination phase, in which the stalk is produced and the spores move up the stalk to form a fruiting body, see Fig. 1.1. Prestalk cells move towards the center of the structure, called Mexican hat because of its shape, where they harden, producing cellulose to give integrity to the stalk, and then die. The ratio of prestalk to prespore cells consists in D. discoideum of 20 percent of the aggregating cell mass [34]. Additionally, the prespore cells develop to become spore cells, which makes them unable to move. Thus, the continued addition of cells to the stalk pushes the spores up the stalk [37].

The fruiting body has two major functions: Firstly, keeping the spore cells from germinating, which is ensured by germination-inhibiting substances inside the sporocarp 
[38]. Secondly, allowing for long range dispersal of the spores, which is enabled via the projection of the sporocarp into cavities in the soil [39]. This makes it more likely to be carried by worms or bugs [40].

For a more detailed description of the entire life cycle of $D$. discoideum refer to the excellent book of Richard H. Kessin [21], which gives an overview of the past research on D. discoideum. A recent review can be found in [41].

\subsubsection{Pattern formation in $D$. discoideum}

An account of pattern formation in $D$. discoideum would be incomplete without describing the signal cascade inside the cell in response to extracellular stimulation of cAMP. In order to explain how pattern formation works, we consider the cell-to-cell interactions in combination to single cell reactions.

\section{cAMP dependent signal cascade}

The active compound in the pattern formation mechanism of D. discoideum cells is cAMP, which the cells produce and react to. The amoebae detect extracellular signals of cAMP using transmembrane proteins called cARs, cAR1 [42], cAR2 [43], cAR3 [44] and cAR4, see Fig. 1.5 [45]. Among these receptors, cAR1 dominates early aggregation cAMP detection, as was shown using a knockout mutant of cAR1 [46]. Without cAR1, D. discoideum cells will neither bind cAMP nor show chemotaxis towards it. In contrast, cAR2 is only produced after the initial aggregation phase and primarily by prestalk cells [43], where it is necessary for tip formation in the aggregate. Similarly, cAR3 is expressed in the mound stage, having an overlapping activation to both cAR1 and cAR2, which might explain why the cAR3 knockout does not show a clear phenotype [44]. Lastly, cAR4 is expressed both in the elongation stage of the mound phase, in which the initial tip is formed, and afterwards [45]. While the cAR4 knockout mutant does show normal early aggregation, slug movement and fruiting body formation are impaired, which shows that cAMP signaling is important even in late stage aggregation. The reason for $D$. discoideum cells having four different cARs at their disposal might be to allow the cells to sense a large range of cAMP concentrations [47]. This is not unlikely, as the extracellular concentration of cAMP increases strongly during development, ranging from about $1 \mathrm{nM}$ to $1 \mu \mathrm{M}$ in the aggregation stage [48] to $10 \mu \mathrm{M}$ or even $100 \mu \mathrm{M}$ [49-51] in the slug and aggregate stage. Indeed, the affinities of the earlier receptors cAR1 and cAR3 to cAMP are much higher than those of the later cAR2 and cAR4 [45].

The signal received by the cARs is transmitted via a $\mathrm{G}$ protein by disassociation of the $\mathrm{G} \beta \gamma$ from the $\mathrm{G} \alpha$ complex. There are a number of genes encoding for different versions of this $\mathrm{G} \alpha$ complex, resulting in complexes that have different effects on the organism. The G $\alpha 2$ complex is crucial for pattern formation, as it passes the cAMP signal from cAR1 on [53]. Another interesting complex is $\mathrm{G} \alpha 9$, which seems to suppress the formation of signaling centers in the aggregation stage, as $\mathrm{G} \alpha 9$ knockouts produce about 3-4 times more aggregates than wildtype (WT) cells [54]. Furthermore, G $\alpha 9$ downregulates the 


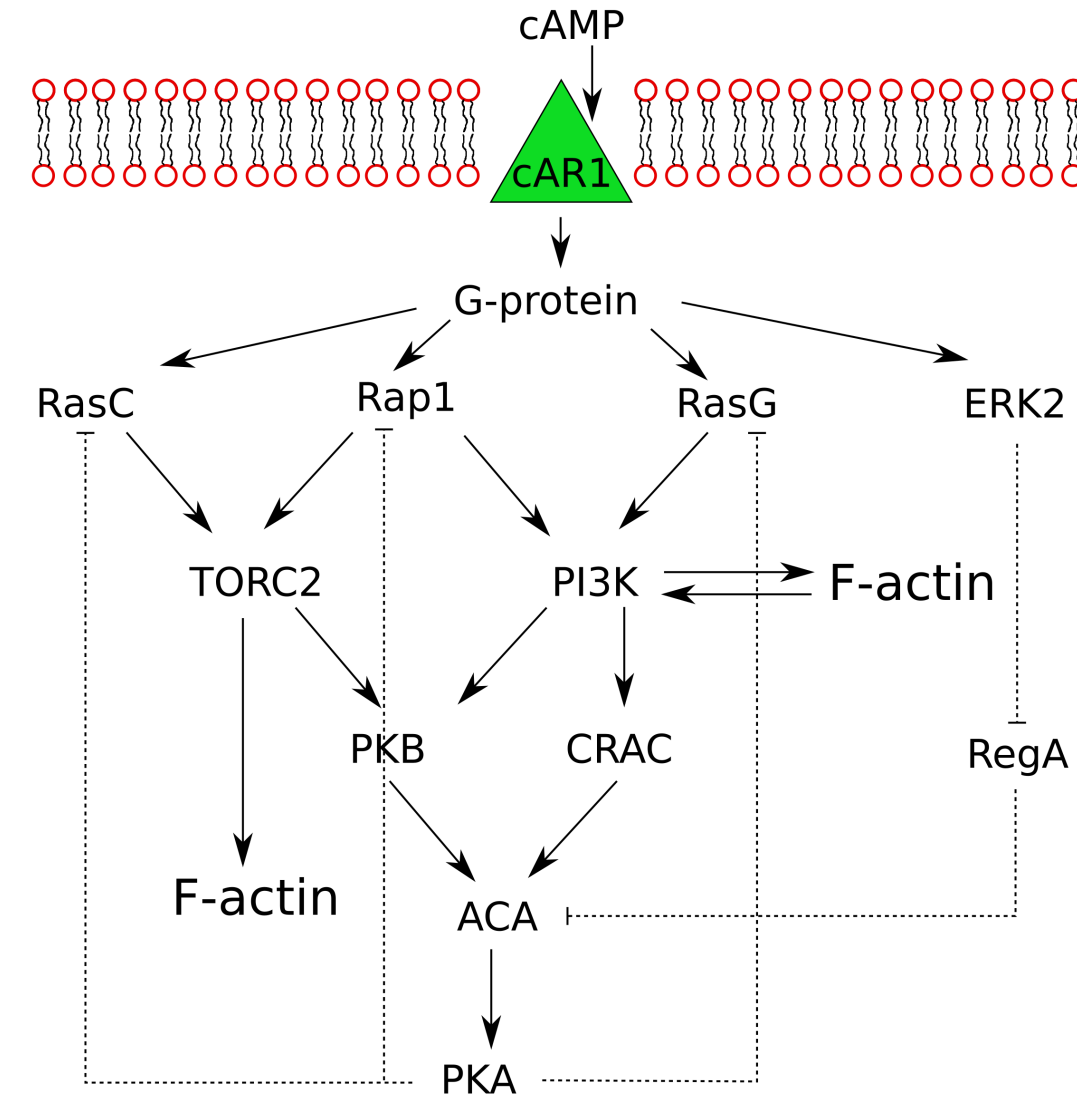

extracellular

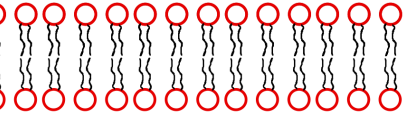

intracellular

Figure 1.5: Overview of the intracellular response of $D$. discoideum cells to extracellular stimulation by increased cAMP levels. Adapted by permission from Springer Nature Customer Service Centre GmbH: Springer, Molecular and Cellular Biochemistry, Caffeine inhibits PI3K and mTORC2 in Dictyostelium and differentially affects multiple other cAMP chemoattractant signaling effectors, A. F. M. Tariqul Islam, Margarethakay Scavello, Pouya Lotfi, Dustin Daniel, Pearce Haldeman and Pascale G. Charest, Copyright 2020, (2019) [52]

cAMP dependent response of the cAR1 - G $\alpha 2$ pathway [55]. Regarding details about the other $\mathrm{G} \alpha$ complex variants refer to the recent review of Devreotes et al [56].

The signal of the G $\alpha 2$ complex is then passed on to a number proteins, see Fig. 1.5. Among these are the two Ras small G-proteins RasC [57], RasG [58] and a homologue of the Ras proteins Rap1 [59], which act as molecular switches. Additionally, the MAP kinase ERK2 is activated [60], which phosphorylates and thus inactivates the intracellular cAMP degrading phosphodiesterase (PDE) RegA, which is also called PDE2 [61,62]. The signal is transferred further by RasG and Rap1 activating PI3K [63,64], which in turn activates PKB and CRAC [65,66]. Furthermore, RasC and Rap1 activate TORC2 [67,68].

Both CRAC and PKB have an activating effect on Adenylate Cyclase of Aggregation stage (ACA) [56,69], which is responsible for the intracellular production of cAMP [70]. Two more adenylate cyclases have been identified in D. discoideum, ACG and ACB, 
however neither ACG nor ACB are important for aggregation [70,71]. The former has been shown to be active during the germination stage and is a sensor for osmolarity, inhibiting spore germination at high osmolarities [72]. Similarly, ACB is active late in development, being necessary for the formation of spores and fruiting bodies [73]. Finally, ACA activates PKA, which inhibits RasC, RasG and Rap1 activity and thus the three pathways for ACA activation, providing an autoinhibitory response of the system [74], as shown in Fig. 1.5.

\section{Cell motility}

Cell motility is a wide field of study, in which $D$. discoideum is studied especially in its chemotactic motility response to cAMP $[75,76]$. The cells have been shown to react in a similar manner to mechanical guidance cues [77], to electric fields [78, 79] and to light [80]. The mechanical response is especially relevant for this work, as there is a response to shear stress induced by fluid flows [81].

Trailing edge (Back)

Leading edge (Front)

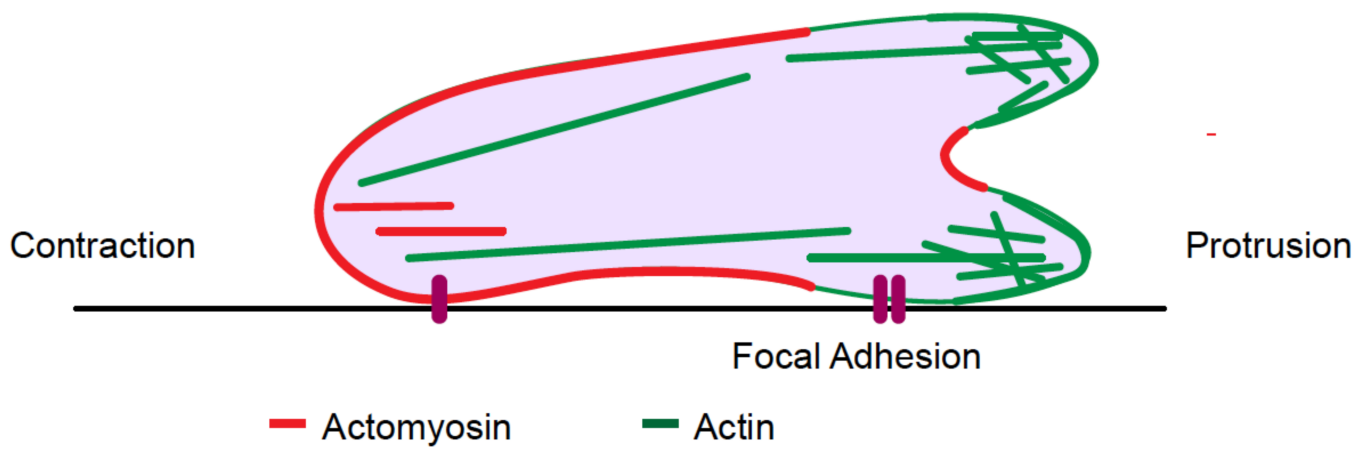

Figure 1.6: Sketch showing the general mechanics of D. discoideum migration. The cells produce protrusions at the cell front by actin polymerisation. These protrusions then are anchored to the surface by adhesion foci. At the back of the cell myosin induces a contraction, thereby dragging the cell back along. Adapted by permission from UPV/EHU Press: The International journal of developmental biology, The excitable signal transduction networks: movers and shapers of eukaryotic cell migration, Dhiman S. Pal, Xiaoguang Li, Tatsat Banerjee, Yuchuan Miao and Peter N. Devreotes, Copyright 2020, (2019). [76]

In general, $D$. discoideum cells move by creating protrusions on the front of the cell and retracting the back of the cell, see Fig. 1.6 [76]. At the leading edge of the cell, F-actin is polymerized, thereby pushing the cell membrane forward. Additionally, the cells adhere to the substrate by forming adhesion bonds and bond clusters, which anchor it to the substrate. The back of the cell, the uropod, is contracted because of increased myosin contractile activity. There are a number of different general migration types cells use, as shown in Fig. 1.7. The main difference between these types of migration is the shape and 
a Macropinosomes

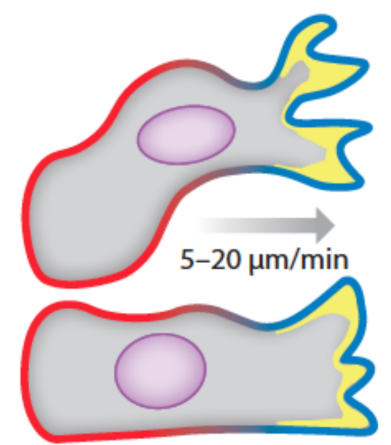

C Blebs

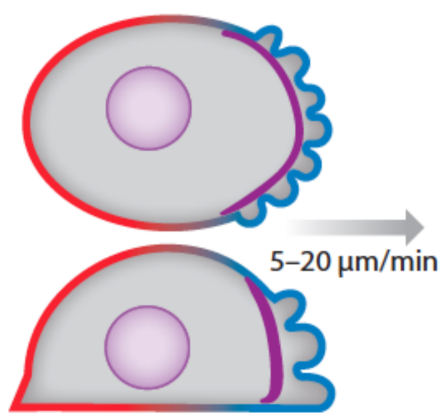

b Pseudopods

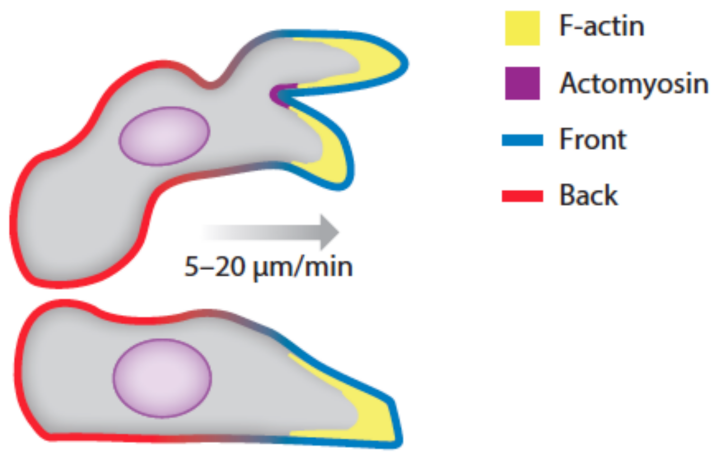

d Lamellipodia

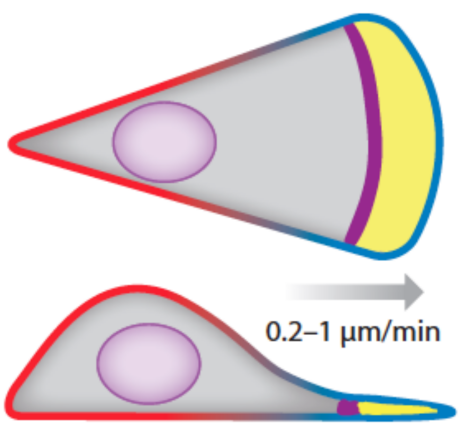

e Collective migration

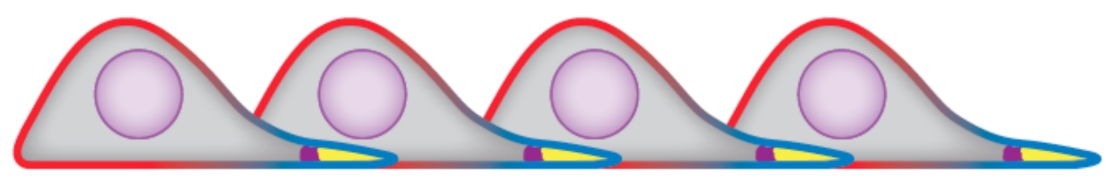

Figure 1.7: Sketch showing different types of cell migration. In all cases the cell membrane is extended in the direction of cell movement. Republished with permission of Annual Reviews, Inc., from Annual Review of Cell and Developmental Biology - Excitable Signal Transduction Networks in Directed Cell Migration, Peter N. Devreotes, Sayak Bhattacharya, Marc Edwards, Pablo A. Iglesias, Thomas Lampert, and Yuchuan Miao, 33, 1, 2017, Copyright 2020; permission conveyed through Copyright Clearance Center, Inc. [56] 
structure of the protrusions the cells produce. During normal migration D. discoideum cells belong either to the class of pseudopod producing cells or to the collective migration class, see Fig. 1.7b,e. However, when the cells detect an increased mechanical reaction, they switch to using bleb based migration [82]. While lamellipodial migration is observed for cells with increased active membrane patches [83], macropinosomes are often observed for axenic strains [84]. Finally, D. discoideum cells can swim, using direct shape changes $[85,86]$.

Considering that $D$. discoideum cells show chemotaxis to cAMP, the signal cascade related to movement is closely linked to the one described above. This is shown by the activation of F-actin in the signal cascade, see Fig. 1.5. However, the signal cascade connected to cell migration is more complicated than shown here, for a detailed review see [76].

\section{From pulses to patterns}

D. discoideum cells will only respond to external concentrations of cAMP if they are above a threshold. This critical concentration depends on the cell state, meaning that while vegetative cells will respond to stimuli in the range of $0.1-10 \mathrm{mM}$, cells in the aggregation stage are much more sensitive and show a response for concentrations in the range of $10^{-1}-10^{2} \mu \mathrm{M}[87,88]$. A stimulus of cAMP above the respective threshold leads to the internal signal cascade described above. This finally results in increased cAMP production inside the cells. cAMP is produced by the enzyme ACA binding to ATP and converting it to cAMP, which is then released into the extracellular medium [89]. As ACA localizes at the back of the cells, cAMP is released to the medium there [90]. Additionally, $D$. discoideum amoebae release vesicles at their back that contain ACA and therefore produce and release cAMP [91]. The response of $D$. discoideum to a cAMP stimulus is transient, in that the cells produce only a certain amount of cAMP independent of the stimulus concentration and only for a limited time of about 3 min [89]. This behavior has been shown in perfusion experiments with long term (20 min) exposure of $D$. discoideum to an external signal [89]. Indeed, the cells respond to differences in the external concentration, as long the concentration is not high enough to saturate cAR1 [92]. However, in chemotactic studies a dependence of migration speed on applied cAMP gradient has been shown [93]. In this study, a microfluidic gradient mixer was used to apply well defined cAMP gradients to D. discoideum cells. Measurements of the chemotactic velocity showed a significant increase in overall motility and velocity for gradients above $\nabla c \approx 10^{-3} \mathrm{nM} / \mu \mathrm{m}$. Both properties decayed rapidly for gradients above $\nabla c>3.3 \mathrm{nM} / \mu \mathrm{m}$.

This type of a transient response is facilitated by the desensitization of cAR1 via phosphorylation of the receptor [94]. Desensitization is a rapid process, starting $20 \mathrm{~s}$ after the stimulus is applied with a half time of 2-3 min [95]. After external cAMP is removed, the receptors resensitize quickly, showing dephosphorylation with a half time of about 2 min [96]. cAMP production and release happens on a similar timescale, starting about 2 min after the signal is applied and lasting for about 3-4 min in total [89].

Since $D$. discoideum amoebae only resensitize after extracellular cAMP is removed or 
has diffused away, the cells produce phosphodiesterases (PDE). These enzymes degrade cAMP, and are released into the extracellular medium by the cells, thus speeding up cAMP removal [49,97]. D. discoideum cells produce seven types of PDE, of which only PDE1, PDE4 and PDE7 degrade extracellular cAMP, while the others degrade intracellular cAMP or cGMP [49]. During the aggregation stage nearly all extracellular cAMP is degraded by PDE1 (also know as pdsA) [49]. For this reason, knockout mutants of PDE1 do not show pattern formation and thus cannot aggregate [98]. Because of its expression pattern and rate constants, PDE4 most likely degrades at least half of the extracellular cAMP in the slug stage [49]. PDE7 seems to be a homologous protein to PDE1. While PDE1 and PDE7 are released into the extracellular medium and can also bind to the membrane, PDE4 is a transmembrane protein [49]. D. discoideum amoebae also regulate the degradation of cAMP using an inhibitor of PDE called PDI that is released into the extracellular medium [99].

The entire signal relay system results in pulses of cAMP being produced. These waves then propagate through the extracellular medium, where other cells relay the waves by producing more cAMP in response to suprathreshold stimulation. Cells that have produced cAMP do not respond to cAMP until their receptors are resensitized. This leads to the absence of the backpropagation of waves and allows for the clear identification of an aggregation center. Moreover, the cells degrade cAMP, leading to a reconstitution of signaling ability. This pulsatile manner of transmitting signals allows the cells to conserve energy in contrast to nonstop cAMP production. Furthermore, the cells can use gradients of cAMP, instead of the actual concentration, to navigate.

D. discoideum cells respond chemotactically to cAMP stimulation, moving towards increasing gradients of the chemical at about $3 \mu \mathrm{m} / \mathrm{min}[100,101]$. Indeed, cells have been shown to be able to detect as little as a $2 \%$ difference of cAMP concentration along the cell length [102]. The amoebae react to fold-changes of the extracellular cAMP instead of the actual concentration [103]. Considering that the cAMP waves are symmetrical, the cells' chemotactic behavior should lead to them moving back and forth without a net movement. However, the cells respond only to the first part of the wave and ignore the gradient at its back. The question of why the cells behave in this way is called the back-of-the-wave problem. An explanation was found in a memory effect in D. discoideum chemotaxis confirmed by flow through experiments [104]. The onset of the cells forming aggregation streams has been interpreted as an instability by coupling a reaction-diffusion model to density fluctuations in response to cAMP [105].

This type of chemotactic response makes the chemical waves accessible by dark-field microscopy, see (section 1.6.2, Fig. 1.19). When a wave reaches a cell, it responds by rounding up and stopping for about half a minute, which results in it scattering relatively little light [106]. Then the cell moves up the gradient, meaning that it extends pseudopodia and thus elongates, which increases the amount of scattered light. Finally, after the wave has passed, the cell will return to small random movements, resulting in an intermediate amount of light scattering. The initial decrease in light scattering can be observed as a dark band, which is followed by a brighter band due to the directed cell movement and finally a return to intermediate light intensity as the cells return to undirected movement. A study employing isotope dilution fluography confirmed 
that the waves of different light intensity correspond to real chemical waves [48]. This was determined using competitive binding of the cells' cAMP and ${ }^{3} \mathrm{H}$-labeled cAMP to protein kinase. Areas with increased cAMP concentration then showed higher activity on an x-ray film.

Furthermore, during the aggregation stage, the cells exhibit not only regular shape changes according to the cAMP wave period but also oscillations in the distance to the substrate, as was shown using electric cell-substrate impedance sensing [107]. Indeed, the cells were also found to transiently form lager cell clusters during aggregation, with the number of clustered cells oscillating at the frequency of the cAMP waves [107].

\subsubsection{Wave characteristics}

To facilitate the overall comparison of pattern formation in D. discoideum to other systems, a concise description of the cAMP waves the cells produce, the patterns that these waves form, and their characteristics shall be given here.

The large scale patterns in D. discoideum are under normal conditions either spiral waves or concentric rings, also called target centers [16,108]. Cell density seems to be a decisive factor in determining which of the two pattern types is produced. At lower cell densities, target centers appear, whereas spirals dominate at higher cell densities [109]. In contrast to other excitable media, the cores of spirals in D. discoideum are stable and do not meander. Spiral waves are normally single armed, with double armed spirals being rare and unstable [108].

The waves themselves move at a speed of $0.3-0.5 \mathrm{~mm} / \mathrm{min}[48,101,110,111]$. A considerable reduction of the wave speed takes place during aggregation, starting from 0.5 $\mathrm{mm} / \mathrm{min}$ and going down to $0.2 \mathrm{~mm} / \mathrm{min}$ [110-112]. The wave speed scales inversely with environment temperature [111]. The wave period is about 6 minutes (between 5 and 8 minutes) initially, but decreases to 3 min during aggregation [101,108]. The spiral period scales with the environmental temperature, following an Arrhenius scaling [111]. For densities below a monolayer (defined as $6.6 \times 10^{3}$ cells $/ \mathrm{mm}^{2}$ ) it was shown that the wave period increases with lower cell density [113]. The wave width shows considerable variation between $0.3-1 \mathrm{~mm}$ and $0.5-0.9 \mathrm{~mm}[48,114]$. The wave length is similarly variable with values between $1-2 \mathrm{~mm}$ but even up to $3 \mathrm{~mm}$ being reported [101,114]

The time from the start of starvation to the onset of pattern formation is about 3-4 hours, while about 7-8 hours pass from starvation to the initialization of aggregation $[115,116]$. An often used technique for the cell preparation is initial starvation in nonnutrient buffer to synchronize the cell state, as well as to induce earlier pattern formation and cell aggregation [116]. This technique can be extended by regularly injecting cAMP drops into the buffer, which speeds up cell development and can rescue ACA deficient mutants [117]. 


\section{Caffeine and its influence on pattern formation}

In 1984 it was shown that caffeine has an inhibitory effect on the production of cAMP in D. discoideum [118]. Brenner \& Thoms discovered that if caffeine was added to the medium of the amoebae in concentrations of $1 \mathrm{mM}$ to $3 \mathrm{mM}$, it inhibited the cAMP dependent activation of ACA and thus cAMP production in vivo. Additionally, they found that the cells' sensitivity to stimulation by cAMP was enhanced, which could be explained by the lower cAMP production of the cells. This led to a lower background concentration of cAMP. Considering that the cells respond to increases in cAMP compared to the background concentration, this then resulted in a lower concentration threshold for a response. Additionally, the drug did not seem to have an adverse effect on the cells' viability, as they continued to show pattern formation and aggregation. Another important factor was the demonstration that the effects of caffeine were easily reversible by removal of the drug from the medium [92].
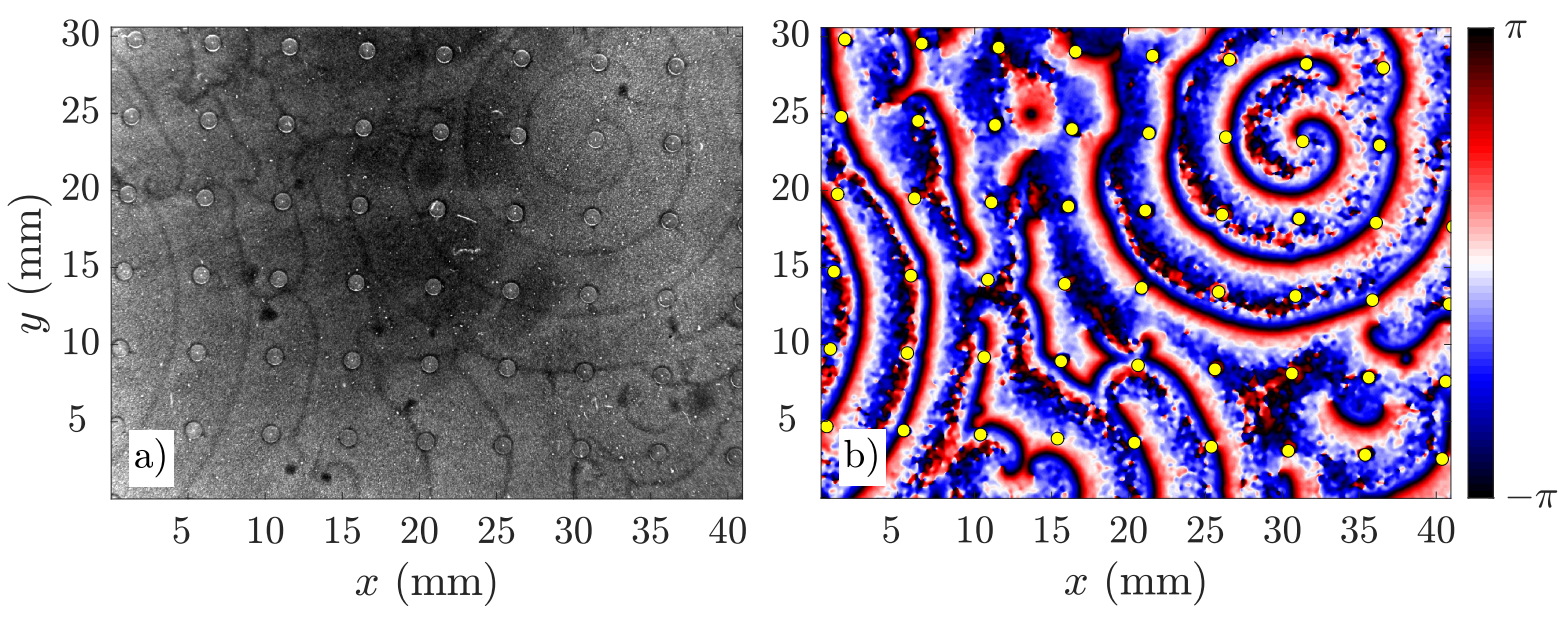

Figure 1.8: Experimental images showing spiral waves in Ax3 aggregation under the addition of $2 \mathrm{mM}$ caffeine and with initial starvation in shaking culture for $4 \mathrm{~h}$. a) shows the original dark-field image with spirals on a PDMS obstacle dish after about $11 \mathrm{~h}$ of starvation. b) shows the phase extracted from a) after image normalization and bandpass filtering, as described in section 1.6.3. In b) the obstacles are shown in yellow. Note that one spiral in the top right corner is pinned to an obstacle and that the unpinned spirals have dark and mostly cell-free spiral cores. [unpublished data]

After Brenners \& Thoms' discovery, many other scholars joined the investigation of the effects of caffeine on pattern formation in D. discoideum. A study in 1989 by Siegert and Weijer showed that the drug had a number of effects on the two-dimensional patterns observed [110]. If cells were plated on agar that included caffeine, it led to an increase in the visibility of the waves in the dark-field microscopy setup that was utilized, without hindering cell aggregation. Moreover, in the presence of caffeine the wave period increased, while the wave speed decreased in a dose dependent manner. Furthermore, the drug induced changes in the shape of the waves in more subtle ways. This resulted 
in a broadening of the dark band of the wave and the introduction of a new thin leading dark band before the bright one, leading to bimodal waves $[108,110]$. The size of the patterns and consequently the size of the aggregation territories was also increased in the presence of caffeine. Finally, the core of spiral waves was shown to be devoid of cells, with its size increasing for higher applied caffeine concentrations, as shown in Fig. 1.8. The phenomenon of a cell-free spiral core was shown to be connected to the movement of the cells on a ring around the spiral core [119]. The effect of caffeine on the wave period was also investigated using electric cell-substrate impedance sensing [120]. In this study average periods were reported: $6.8 \mathrm{~min}$ for the case of no caffeine, $16.5 \mathrm{~min}$ at $1.5 \mathrm{mM}$ caffeine and $25.3 \mathrm{~min}$ at $3.1 \mathrm{mM}$ caffeine.

More recently, researchers have shown several ways for caffeine to influence the size of aggregates in D. discoideum [121]. It was shown that the application of caffeine to cells plated on agar decreases the average aggregate size in a dose dependent manner, contradicting earlier work mentioned above [110,121]. Furthermore, cell-to-cell adhesion increases in cells treated with caffeine [121]. This may have led to cells aggregating without showing streaming. It was also shown that caffeine influences the mechanism D. discoideum uses to determine local cell density, which might impact aggregate size as well [121].

However, caffeine does not only influence aggregate size but also motility. A speed up of cells from $5.07 \pm 0.61 \mu \mathrm{m} / \mathrm{min}$ without caffeine to $6.49 \pm 0.59 \mu \mathrm{m} / \mathrm{min}$ under the addition of $2 \mathrm{mM}$ was measured [121]. In addition, a decrease of pdsA expression was shown in cells treated with $3 \mathrm{mM}$ caffeine after 6 hours [121]. Researchers are still currently studying the effects of caffeine on the signaling cascade at a molecular level. Islam et al showed that a number of processes in the signal cascade changed in cells under the effect of caffeine [52]. This includes a downregulation of the response in PI3K and $\mathrm{mTORC} 2$ in reaction to cAMP stimulation. Consequently, the activity of PKB and PKA was found to decrease as well.

The case of the MAP kinases is somewhat more challenging. While ERK1 was found to be less active under the addition of caffeine, ERK2 showed different results for Ax2 and Ax3 cells. The first showed reduced activity in response to cAMP stimulation, while the second had a higher activity. Additionally, the response of RasG and Rap1 was shown to be increased in caffeine treated cells. Finally, the authors discovered that a number of factors involved in the chemotactic response were influenced as well. An overview of the effects of caffeine on the signal cascade is shown in Fig. 1.9. Based on these observations, it seems that direct inhibition of mTORC2 and PI3K activity leads to downstream downregulation of ACA and thus cAMP production in caffeine treated cells. For Ax2 cells, the decrease in ERK2 activity leads to an increase in intracellular cAMP degradation via RegA damping the cAMP response. However, the entire picture is more complicated, as there is an increase in activity of upstream factors in RasG and Rap1 and the cells are impacted in the chemotactic signaling pathway as well.

Since we used caffeine to suppress ACA activity in the experiments described in chapter 4 , we studied the response of $D$. discoideum cells to caffeine in our system. Considering that the cells' environment could be important as well, we studied Ax2 cells both on $1.5 \%$ Phosphatebuffer agar and under a liquid layer in a flat PDMS setup. For all 


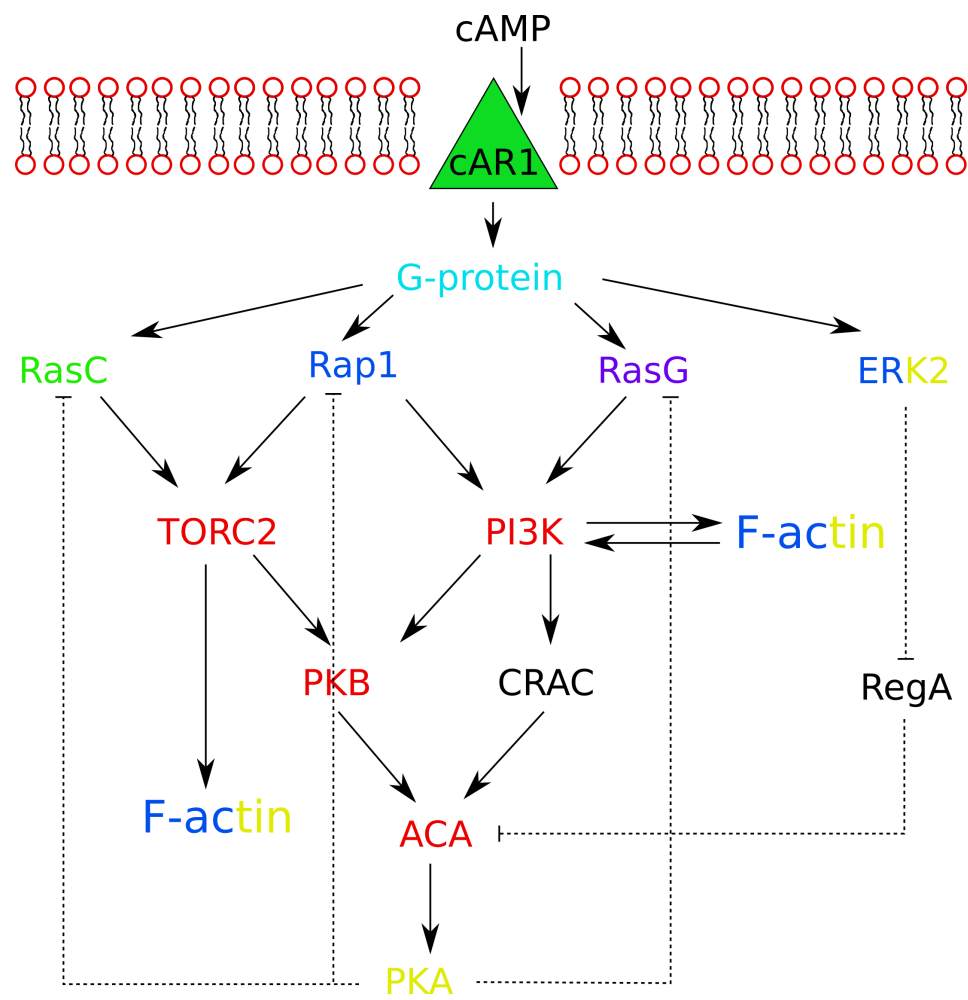

extracellular

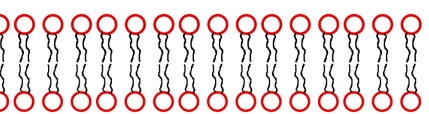

intracellular

effect of caffeine: पIIIIII

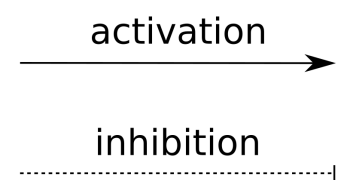

Figure 1.9: Overview showing the effect of caffeine on the intracellular response of D. discoideum cells to extracellular stimulation by increased cAMP levels. Adapted by permission from Springer Nature Customer Service Centre GmbH: Springer, Molecular and Cellular Biochemistry, Caffeine inhibits PI3K and mTORC2 in Dictyostelium and differentially affects multiple other cAMP chemoattractant signaling effectors, A. F. M. Tariqul Islam, Margarethakay Scavello, Pouya Lotfi, Dustin Daniel, Pearce Haldeman and Pascale G. Charest, Copyright 2020, (2019) [52]

experiments the cells were prepared as described in section 1.6.1 and image processing and analysis was done as described in section 1.6.3. We did experiments at caffeine concentrations of $0 \mathrm{mM}, 0.5 \mathrm{mM}, 1 \mathrm{mM}, 2 \mathrm{mM}$ and $3 \mathrm{mM}$. Note that all cells were initially starved for $4 \mathrm{~h}$ in buffer with or without caffeine before the experiment was started. We included the effect of caffeine in our simulations by reducing the affinity of ATP to ACA, see chapter 4 .

The change of the cells' period during the experiments for different caffeine concentrations and in the different environments is shown in Fig. 1.10. There seems to be an influence of the cell environment, since the periods on PDMS are lower for $0 \mathrm{mM}$ and 0.5 $\mathrm{mM}$ than their counterparts on agar but higher at all other concentrations. Additionally, one can see that the period generally increases during the experiment, with the case of $0 \mathrm{mM}$ on PDMS being an exception. 


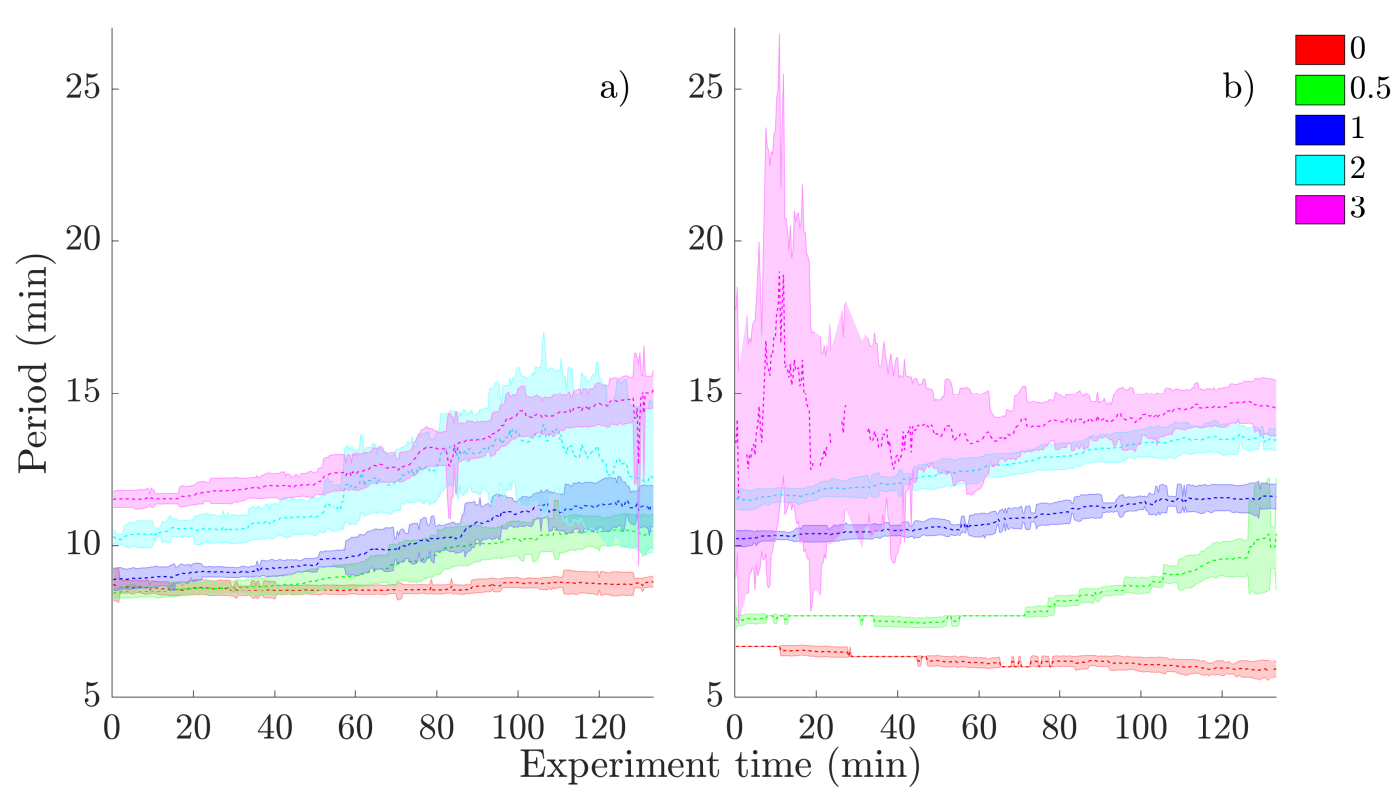

Figure 1.10: Period as a function of time for different caffeine concentrations on either a) a $1.5 \% \mathrm{~Pb}$ agar dish or b) a flat PDMS setup. Cells were starved in shaking culture for $4 \mathrm{~h}$ before the start of the experiment. Both while in starvation and during the experiment the same caffeine concentration was applied. Higher caffeine concentrations seem to lead to higher periods. During the experiment the period increases with time. The legend on the right side shows the applied caffeine concentration in mM. [unpublished data]

\subsubsection{Notable strains}

Over the years, a number of different strains of $D$. discoideum have been isolated and documented. A defining feature that unites most of the strains used in research as of today is their axenic nature [122]. This means that the cells do not need to be kept in co-culture with bacteria for nutrients. Instead, they have been grown to be able to absorb nutrients suspended in the medium they live in by internalizing it in vacuoles. Generally, this process is called pinocytosis, but $D$. discoideum has been shown to absorb relatively large vacuoles, which led to the name macropinocytosis being termed [123].

Bacteria feeding strains were developed to be axenic simply by allowing them to grow in an appropriate medium. This led to a number of mutations, enabling the cells to grow well even without bacterial food. The earliest example for this method is the strain Ax1, which was developed by Sussman \& Sussman in 1967 [124]. While the Ax1 strain has been lost in the meantime, a derivative of it, Ax2 is still used today. Ax2 was selected from descendants of Ax1, which grew in a medium that needed less ingredients [125]. This strain was used mainly in our experiments, see chapters 2 and 4 . Another line of strains stems from NC-4, which was isolated by Erdos et al in 1973 [126]. As for Ax2, descendants for NC-4 were selected for their ability to grow in the reduced medium, 
which resulted in the strain Ax3 [127].

One of the biochemical advantages of studying $D$. discoideum is the relative ease with which specific genes can be deactivated in the organism to understand the role of the gene and the protein it encodes for. An example of this is the $\mathrm{pdsA}^{-}$strain, in which the gene responsible for the production of PDE1 has been deactivated [98]. While pds $\mathrm{A}^{-}$cells still produce cAMP, they lack the main PDE to degrade extracellular cAMP, resulting in an inability to produce waves and thus aggregate. We used this cell line in a study, in which we used a fresh fluid flow to substitute PDE degradation, see chapter 3. 


\subsection{Reaction-Diffusion systems}

Reaction-diffusion systems have received considerable attention in the field of biology to understand pattern formation. In these systems, spatial isotropy is broken through multiple species of chemicals, which diffuse through space and react with each other locally. The defining feature for pattern formation is that the chemicals' concentrations are separated either in time or in space. This can happen through different diffusive lengths or by means of different time scales for the reactions. The cAMP waves of $D$. discoideum belong to this class of systems. The study of reaction-diffusion systems goes back to 1952, when Turing proposed that morphogenesis might be based on chemical systems of this type [5]. Turing was far ahead of his time and the experimental field, since until the 1950s the idea of a homogeneous chemical reaction oscillating autonomously was alien to the research community.

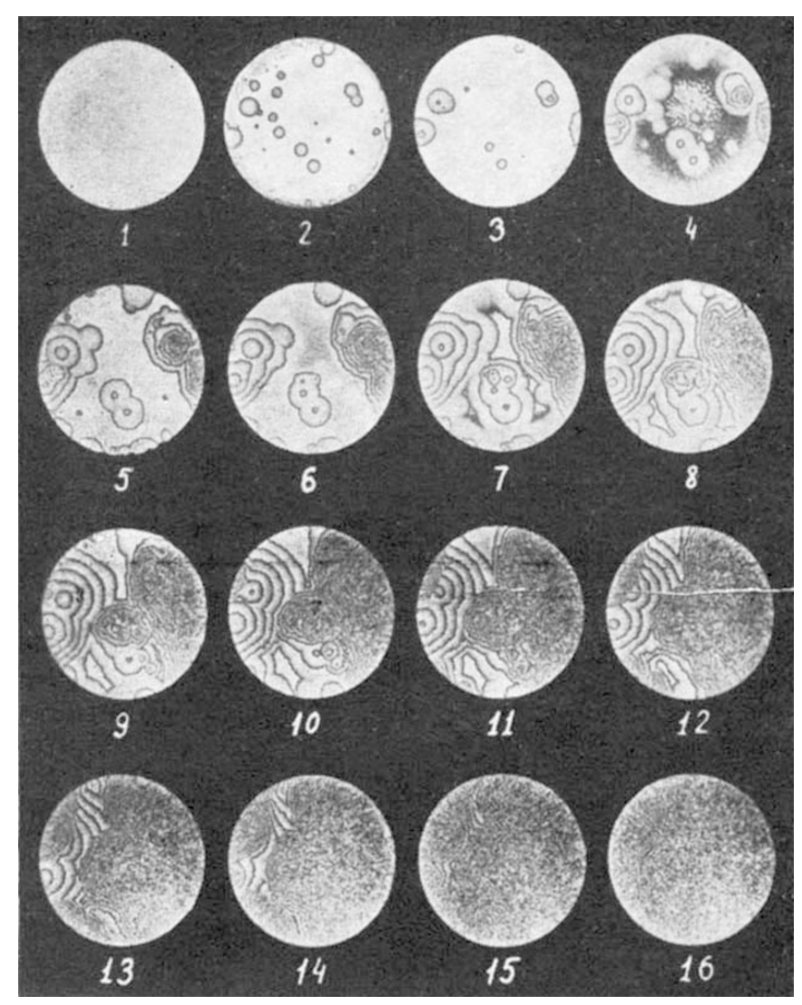

Figure 1.11: Wave patterns in the BZ reaction, showing target patterns. The changes in brightness are due to differences in concentration of oxidized ferroin (Fe(1,10-phenantroline $\left.)_{3}\right)$. This chemical is used as a catalyst and an indicator for the oscillations of bromate and bromomalonic acid. One photo taken every minute. Ring diameter is $100 \mathrm{~mm}$. Reprinted by permission from Springer Nature Customer Service Centre GmbH: Nature, Nature, Concentration Wave Propagation in Two-dimensional Liquid-phase Self-oscillating System, Zaikin, A. N. and Zhabotinsky, A. M., Copyright 2020, (1970). [128]

When Belousov discovered an oscillating reaction system in 1951, it was rejected based 
on the grounds that his discovery was "quite impossible, and would merit publication only if accompanied by a demonstration that existing theory was flawed" [129]. This was due to the idea that Belousovs findings contradicted the second law of thermodynamics [130]. Interestingly, the main point was that Belousov claimed that his reaction happened in a homogeneous system, since periodic reactions in heterogeneous systems had been accepted by the scientific community [130]. The system that Belousov had discovered consisted of Bromite dissolved in citric acid and ceric ions used as a catalyst. The reaction rhythmically changed from transparent to yellow for up to an hour [12]. Since the Russian research community reacted in a dismissive way to Belousovs findings, the reaction was not studied by a broader community until later. In 1961 Zhabotinsky, a graduate student at the time, started researching the original formula. Zhabotinsky then published an article on the reaction in 1970, see Fig. 1.11 [128]. Later, after Belousovs contribution was made public in the west, the name was changed to BelousovZhabotinsky reaction (BZ reaction).

The entire reaction cascade of the BZ reaction is quite complicated, involving many bromate derivatives [131]. However, on a simplified level the reaction can be understood as a two step process $[132,133]$. That is initially the catalyst $\mathrm{Fe}(\mathrm{phen})_{3}{ }^{2+}$ is oxidized by $\mathrm{HBrO}_{3}$, producing $\mathrm{Br}^{-}$. However, the production of $\mathrm{Fe}$ (phen) $)_{3}{ }^{3+}$ is inhibited by high $\mathrm{Br}^{-}$levels. As $\mathrm{Fe}$ (phen) ${ }_{3}{ }^{3+}$ is reduced by bromomalonic acid and its derivatives, its concentration starts falling. This means, that the concentration of the inhibitor $\mathrm{Br}^{-}$ reduces as well. When both are low enough, the rapid oxidation of $\mathrm{Fe}(\mathrm{phen})_{3}{ }^{2+}$ starts again. A reaction formula is given here:

$$
\begin{aligned}
\mathrm{HBrO}_{3}+\mathrm{HBrO}_{2} & \longrightarrow 2 \mathrm{BrO}_{2}{ }^{\bullet}+\mathrm{H}_{2} \mathrm{O} \\
\mathrm{H}_{+}+\mathrm{BrO}_{2}{ }^{\bullet}+\mathrm{Fe}(\text { phen })_{3}{ }^{2+} & \longrightarrow \mathrm{Fe}(\text { phen })_{3}^{3+}+\mathrm{HBrO}_{2} \\
\mathrm{HBrO}_{2}+\mathrm{H}^{+}+\mathrm{Br}^{-} & \longrightarrow 2 \mathrm{HOBr} \\
2 \mathrm{H}^{+}+\mathrm{BrO}_{3}{ }^{-}+\mathrm{Br}^{-} & \longrightarrow \mathrm{HBrO}_{2}+\mathrm{HOBr} \\
\mathrm{HBrO}_{2}+\mathrm{HBrO}_{2} & \longrightarrow \mathrm{HOBr}+\mathrm{BrO}_{3}^{-}+\mathrm{H}^{+}
\end{aligned}
$$

Initially, the Brusselator shall be considered as an example of a simple pattern forming model. The Brusselator is a classical inhibitor-activator two-component system, akin to the proposition of Turing. While the origins of the Brusselator can be traced back as far as 1956, the concise version was proposed as a model for reaction diffusion systems in the 1973 by Tyson $[134,135]$. It can also be seen as a model for the oscillations in the BZ-reaction discussed above. The model describes the interaction of two chemicals in one dimension diffusing and reacting following two coupled partial differential equations:

$$
\begin{aligned}
& \partial_{t} u=a-(b+1) u+u^{2} v+D_{u} \partial_{x}^{2} u \\
& \partial_{t} v=b u-u^{2} v+D_{v} \partial_{x}^{2} v
\end{aligned}
$$

The variables $u$ and $v$ are intermediates in an autocatalytic chemical reaction that can be described as [134]: 


$$
\begin{aligned}
a & \longrightarrow u \\
b+u & \longrightarrow v+d \\
2 u+v & \longrightarrow 3 u \\
u & \longrightarrow e
\end{aligned}
$$

The entire reaction is based on two input chemicals $a$ and $b$ that react, forming the intermediates $u$ and $v$ only to end as the output chemicals $d$ and $e$. The only parameters missing in Eqs. 1.1 are then the two diffusion constants $D_{u}$ and $D_{v}$ of $u$ and $v$.
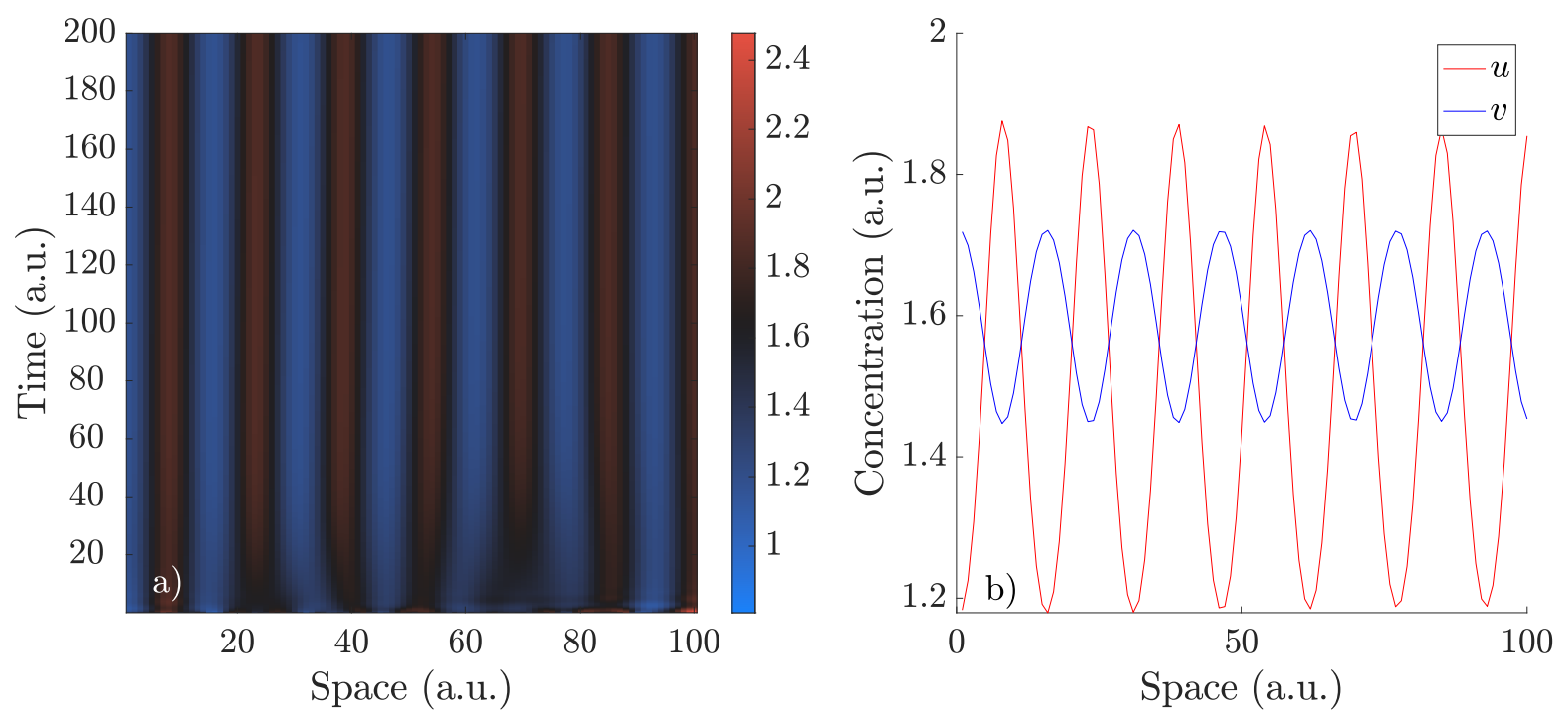

Figure 1.12: Stripe pattern in a one-dimensional Brusselator model, as described by Eqs. 1.1. Parameters used: $a=1.5, b=2.4, D_{u}=2.8, D_{v}=22.4$, for $N=100$ points starting at steady state $u=a, v=b / a+$ random white noise of order $1 / 10$. Integrated with explicit Euler method. a) shows the behavior of $u$ as a function of time. b) shows both $u$ and $v$ at the last integration step as a function of space. Colormap is from [136].

The Brusselator has a unique steady state at $(u, v)=(a, b / a)$. This state is unstable to small perturbations, if the parameters are chosen adequately (see [137, pg. 105 ff.]). Under these conditions, the system produces regular stripes, showing clear Turing patterns, see Fig. 1.12. In this case the separation between the two chemicals' concentrations is implemented via the difference in diffusion coefficients.

Comparing this version of the model to the BZ reaction discussed before, one can see that it is similar, though reduced in complexity. The system corresponds to a number of oscillators, coupled via diffusion, that are stationary in space. Experimentally, this translates to performing an experiment in an undisturbed medium. However, the reactants can be kept under constant stirring conditions as well. This balances the chemical concentrations in space, meaning that the diffusive fluxes disappear $\partial_{x}^{2} u=$ 
$\partial_{x}^{2} v=0$. Under these conditions, the spatial component of the system can be neglected. It shows pattern formation nonetheless, as the system shows oscillations in time, see Fig. 1.13. The system is again initialized close to the stationary point. A small perturbation from this point grows over time, showing the steady points instability. In contrast to the version with diffusion considered above, the system now does not settle in any point in state space. Instead, it moves continuously on a stable and attractive closed orbit around the unstable steady point.
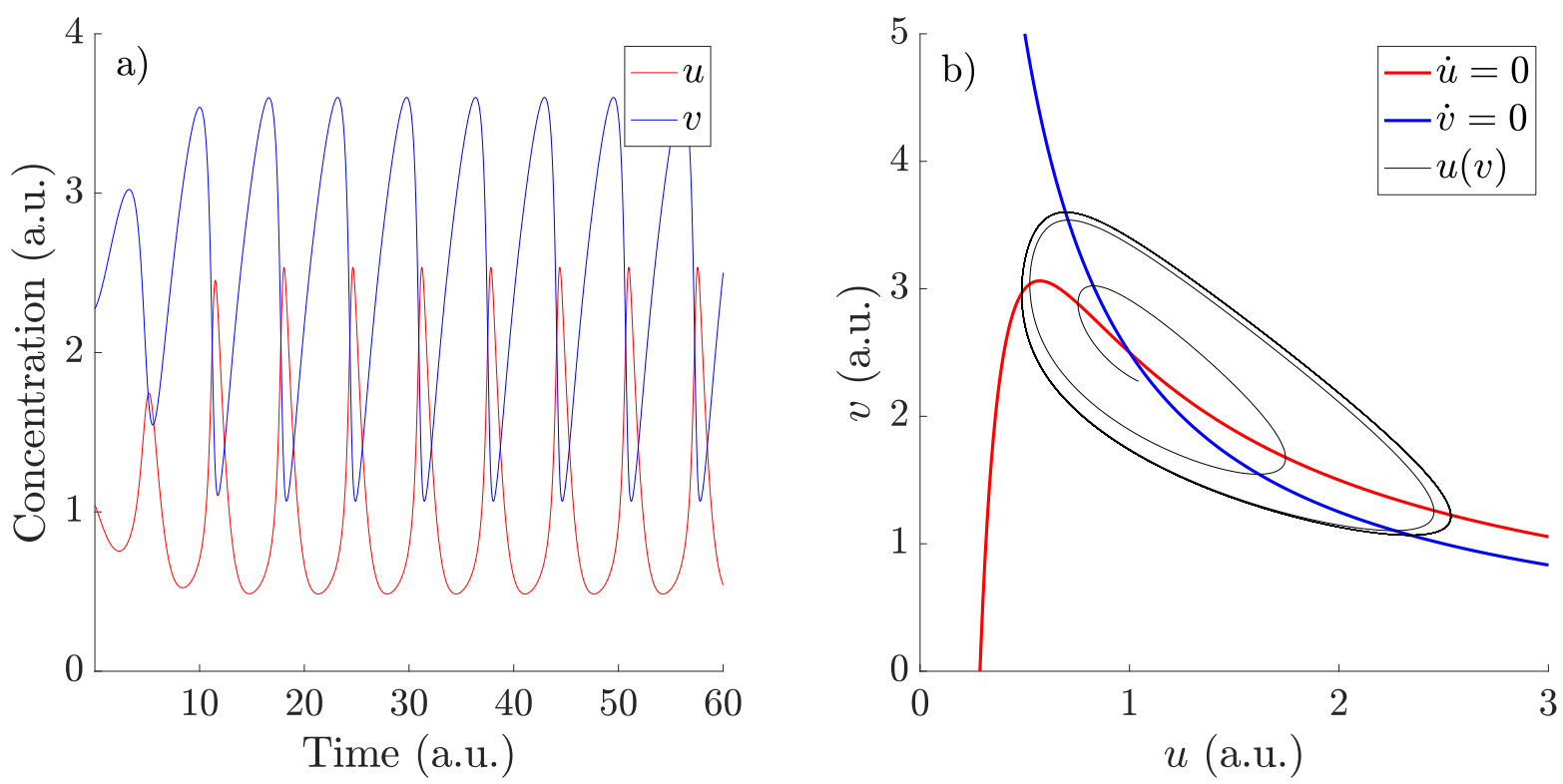

Figure 1.13: Oscillations in a one-dimensional Brusselator model, as described by Eqs. 1.1, neglecting diffusion. Parameters used: $a=1, b=2.5$ starting at steady state $u=a, v=b / a+$ random white noise of order $1 / 10$. Integrated with explicit Euler method. a) shows both $u$ and $v$ as a function of time. b) shows the nullclines of $u$ and $v$ and $u$ as a function of $v$.

The two component Brusselator model can show both Turing patterns and continuous bulk oscillations, but for the formation of traveling waves, it has to be extended to three variables $[5,138]$. The third variable $w$ can be interpreted as a byproduct of the main reaction. This substance is inactive and therefore unavailable to the main reaction. The extended equations are:

$$
\begin{aligned}
\partial_{t} u & =a-(b+1) u+u^{2} v-c u+d w+D_{u} \partial_{x}^{2} u \\
\partial_{t} v & =b u-u^{2} v+D_{v} \partial_{x}^{2} v \\
\partial_{t} w & =c u-d w+D_{w} \partial_{x}^{2} w
\end{aligned}
$$

Note that the new chemical $w$ diffuses rapidly $D_{w}>D_{v}>D_{u}$ to enable another level of spatial separation. Again variables $u, v, a$ and $b$ denote chemical concentrations based on the same reaction that was considered for the two component Brusselator. In 
addition, $c$ and $d$ are the reaction rates connected to the transformation of $u$ to $w$ and its inverse reaction $u \stackrel{\mathrm{c}}{\rightleftharpoons \mathrm{d}} w$.

Integrating this system starting close to the stationary point, shows local damped oscillations as shown in Fig. 1.14a) as a timeline for $u$. However, this behavior is a transient, as can be seen in phase space in Fig. 1.14b). On longer time scales the system produces traveling waves, as shown in Fig. 1.14c).
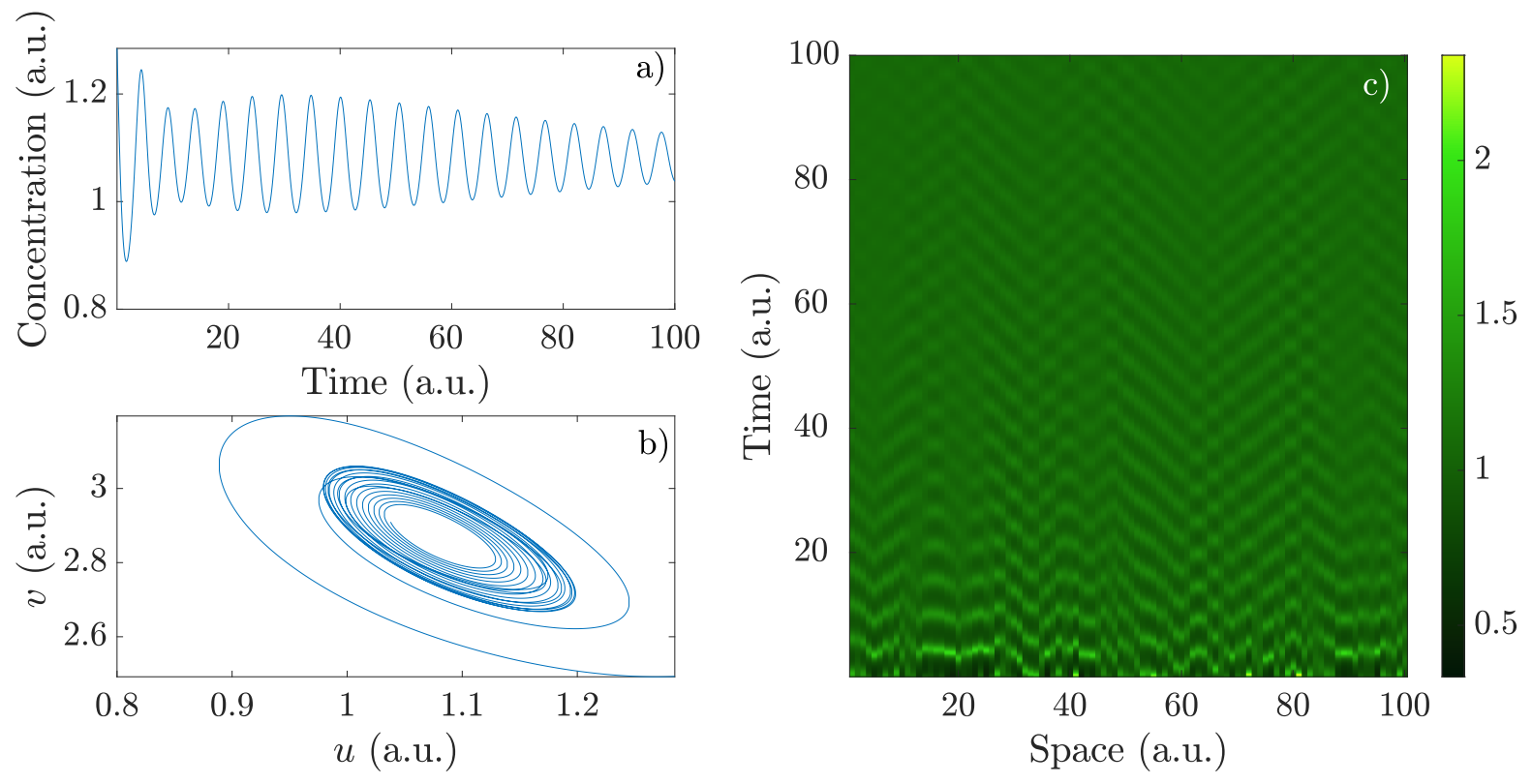

Figure 1.14: Traveling waves in a one-dimensional extended Brusselator model, as described by Eqs. 1.2. Parameters are: $a=1.08, b=3.1, D_{u}=0.01$, $D_{v}=0.1, D_{w}=1$, for $N=100$ points starting at steady state $u=a$, $v=b / a, w=a c / b+$ random white noise of order $1 / 10$. Integrated with explicit Euler method. a) shows $u$ as a function of time for a single point in the middle of the region. b) shows $v$ as a function of $u$ for the same point. c) shows $u$ for the entire system as a function of time. Colormap is from [136].

A rather involved formalism was necessary for producing waves in an oscillatory medium. A simpler approach is to use spatial variations in the oscillation period. If a spot oscillates at a higher frequency than the surrounding medium, it will produce traveling waves. In a two-dimensional system these correspond to centered rings, also called target patterns. This type of spatial variation of parameters is not uncommon in natural systems and has been proposed to explain target center formation in the BZ reaction as early as 1980 [139]. 


\subsection{Excitable Media}

The group of systems known as excitable media differs in a central way from the systems considered before. Until now the stationary points of the models were linearly unstable. This can be seen in that pattern formation was observed after slightly moving the system away from its resting state. In contrast to this, the fixed points for excitable media are linearly stable and require a stimulation of a certain magnitude to show a nonlinear response.

In the 1950s Hodgkin and Huxley pioneered the physical research on nerve and brain function, measuring the ion flows within the giant squid axon [140]. After direct measurements of the ionic currents, they also proposed a model to describe the transmembrane voltages and currents [141]. Electric signals in nerves have a number of features similar to the waves found in $D$. discoideum. The signals are of a pulsatile nature, consisting of short packages of current traveling along the fiber. A pulse will only be relayed if it is above a certain threshold. Once perturbed from the stable fixed point, a fast large excitation is seen, followed by a slow return to the stationary point. The refractory period here is given by the need for the transmembrane voltage to recover. After proposing the initial model, scientists started trying to reduce it to a more compact form, without losing the defining features. Leading in this were FitzHugh who reduced the model considerably and Nagumo who translated it into an electrical circuit [142-144].

The FitzHugh-Nagumo model can be described by two coupled variables $u$ and $v$. The main variable is $u$, which represents the membrane potential. The second variable $v$ is an artificial recovery variable that represents the effects of ionic currents into the cell that restore the membrane potential. Only the potential diffuses based on the diffusion operator $\nabla^{2}=\partial_{x}^{2}+\partial_{y}^{2}$ with diffusion constant $D_{u}$. The equations are reminiscent of the Brusselator considered earlier in that they are two reactions coupled with diffusion:

$$
\begin{aligned}
& \partial_{t} u=\left(u-\frac{u^{3}}{3}-v\right) \nu^{-1}+D_{u} \nabla^{2} u \\
& \partial_{t} v=u+a-b v
\end{aligned}
$$

The $\nu^{-1}$ factor is a crucial difference between the FitzHugh-Nagumo model and the Brusselator, as it determines the relative timescale of the two reactions. To represent a quick spike, followed by a slow membrane repolarization, $\nu<1$ is chosen, slowing down the reaction in $v$ in comparison to the one in $u$. If the system is perturbed strongly enough from the linearly stable fixed point, an excitation pulse is generated, as shown in Fig. 1.15a). Note that the dynamics are very rapid and the system returns to the resting state afterwards. The difference between sub- and supra-threshold stimulations are shown in Fig. 1.15b). For a small perturbation from the stationary point, shown in magenta, the system relaxes back to the resting state very quickly, following the path $(5 \rightarrow 6)$. If the system is perturbed above the threshold, the variables perform a longer orbit, shown in black. Along the path there are two distinct dynamics. The parts of the path connecting from one nullcline to the other $(1,3)$ are much faster than the dynamics along the nullclines $(2,4)$. This explains the fast nonlinear response in the pulse and then the slow relaxation back to the resting state. 

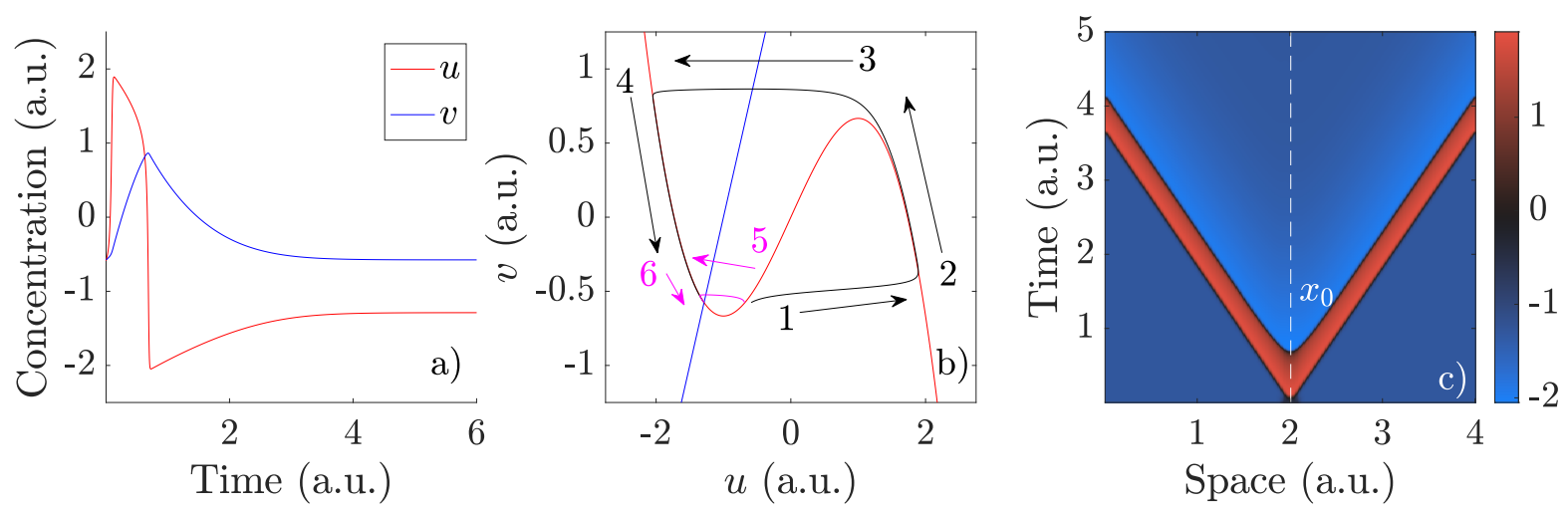

Figure 1.15: Traveling waves in a one-dimensional FitzHugh-Nagumo model, as described by Eqs. 1.3. Parameters used: a-c): $a=1, b=0.5, \nu=0.02$, a-b) $D_{u}=0$ c) $D_{u}=1$, for a-b) $N=5$ c) $N=400$ points at space discretization of $d x=10^{-2}$. Integrated with explicit Euler method for a time of $T=20$. The system has a steady point at $\left(u_{s}, v_{s}\right)=(1.2879,-0.57582)$. a) shows an excitation pulse in $u$ and $v$ as a function of time for a single point in the middle of the region, which was initiated at $\left(u_{s}, v_{s}\right)+(0.7,0)$. b) shows the nullclines of $u$ in red and $v$ in blue and the excitation behavior. The black line corresponds to a supra-threshold pulse, initiated at $\left(u_{s}, v_{s}\right)+(0.7,0)$. The magenta line shows a sub-threshold pulse, initiated at $\left(u_{s}, v_{s}\right)+(0.6,0)$. c) shows two traveling waves in $u$ for the entire system as a function of time. All points initialized at $(u, v)=\left(u_{s}, v_{s}\right)$ except for a stripe of length 0.2 centered around the point denoted as $x_{0}$, which started above threshold at $\left(u_{s}, v_{s}\right)+(0.7,0)$. Colormap is from [136].

In the presences of diffusion, a supra-threshold stimulation is applied in the middle of the simulation domain. This pulse then spreads through the system by diffusion and triggers two traveling waves moving to the right and the left respectively, see Fig. $1.15 \mathrm{c})$. Only two waves are initiated, since the system itself is excitable and only a single perturbation was applied. Note that waves in an excitable medium annihilate when they meet each other or the system boundary. This means that any wave initialized in the system will disappear after some time. Therefore an excitable system has to be stimulated repeatedly in order to continuously generate waves.

The two-dimensional equivalent to a pulse spreading from one point through the system is a ring wave. As was mentioned above, a single excitation will only lead to one pulse. A common way of inducing a wave train is setting some parts of the system to be oscillatory, while most of it stays excitable. The oscillatory points will then repeatedly induce waves, which can spread through the system. An example of this is shown in Fig. 1.16b). Note that oscillatory cells will follow a stable limit cycle in phase space, as shown in Fig. 1.16a).

However, there is another stable wave pattern in excitable media, the spiral wave, as shown in Fig. 1.17. The spiral wave is an excitation pulse that ends in a point 

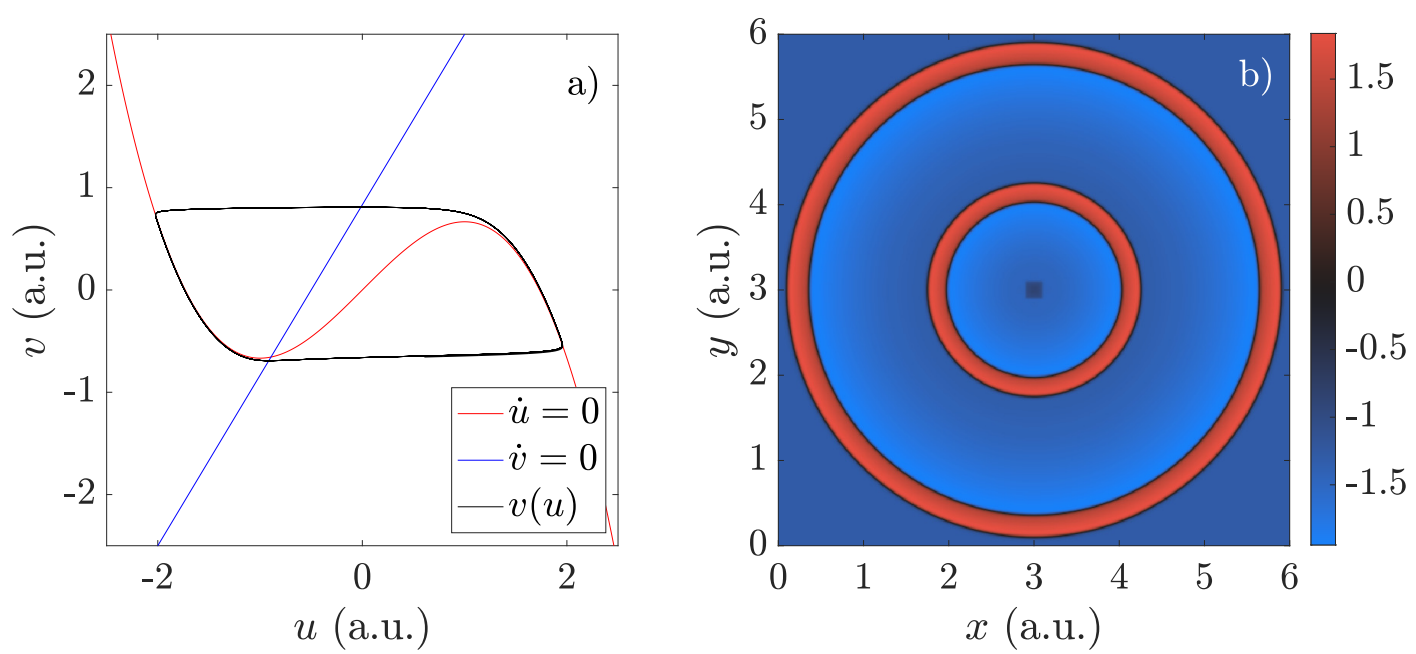

Figure 1.16: Target center in a two-dimensional FitzHugh-Nagumo model, as described by Eqs. 1.3. Parameters used: a) $a=0.5, b=0.6, \nu=0.02, D_{u}=0$, b) everywhere $a=1, b=0.5, \nu=0.02, D_{u}=10^{-2}$, except for a cube of $0.2 \mathrm{x} 0.2$ size in the middle of the domain, which was set to be oscillatory at $a=0.5, b=0.6, \nu=0.02, D_{u}=10^{-2}$, for a) $N=1$ b) $N=600$ points at space discretization of $d x=10^{-2}$. Integrated with explicit Euler method for a time of a) $T=20 \mathrm{~b}) T=50$. a) shows the nullclines for $u$ and $v$ for the oscillatory case and a phase trajectory of an oscillatory point. The equivalent plot for the excitable case is shown in Fig. 1.15b). In b) the initiation of a target center by a cluster of oscillatory points is shown in the field of $u$. All points are excitable except for a $0.2 \times 0.2$ cube in the middle of the domain. This cube oscillates and thereby induces concentric waves that travel through the excitable domain. All points are initiated at the steady state $\left(u_{s}, v_{s}\right)=(-1.2879,-0.57582)$. Colormap is from [136].

called spiral tip. This tip moves on a trajectory through the excitable medium and thus makes the spiral intrinsically stable. For a closed circular orbit, the area enclosed by this trajectory is called a spiral core. The initiation of spirals in excitable media is dependent on wave fragments. A fragment that ends within the medium and touches a part of the medium that is excitable, will move into that part and start curling up into a spiral. This process is shown in Fig. 1.17a-c). First a planar wave front is initiated and allowed to travel through the system. After the planar wave is about in the center of the system, half of the domain is set to the steady state, thereby cutting the wave off. This broken wave fragment then starts curling up onto itself, which leads it to form a spiral wave, shown in Fig. 1.17c) and d). 


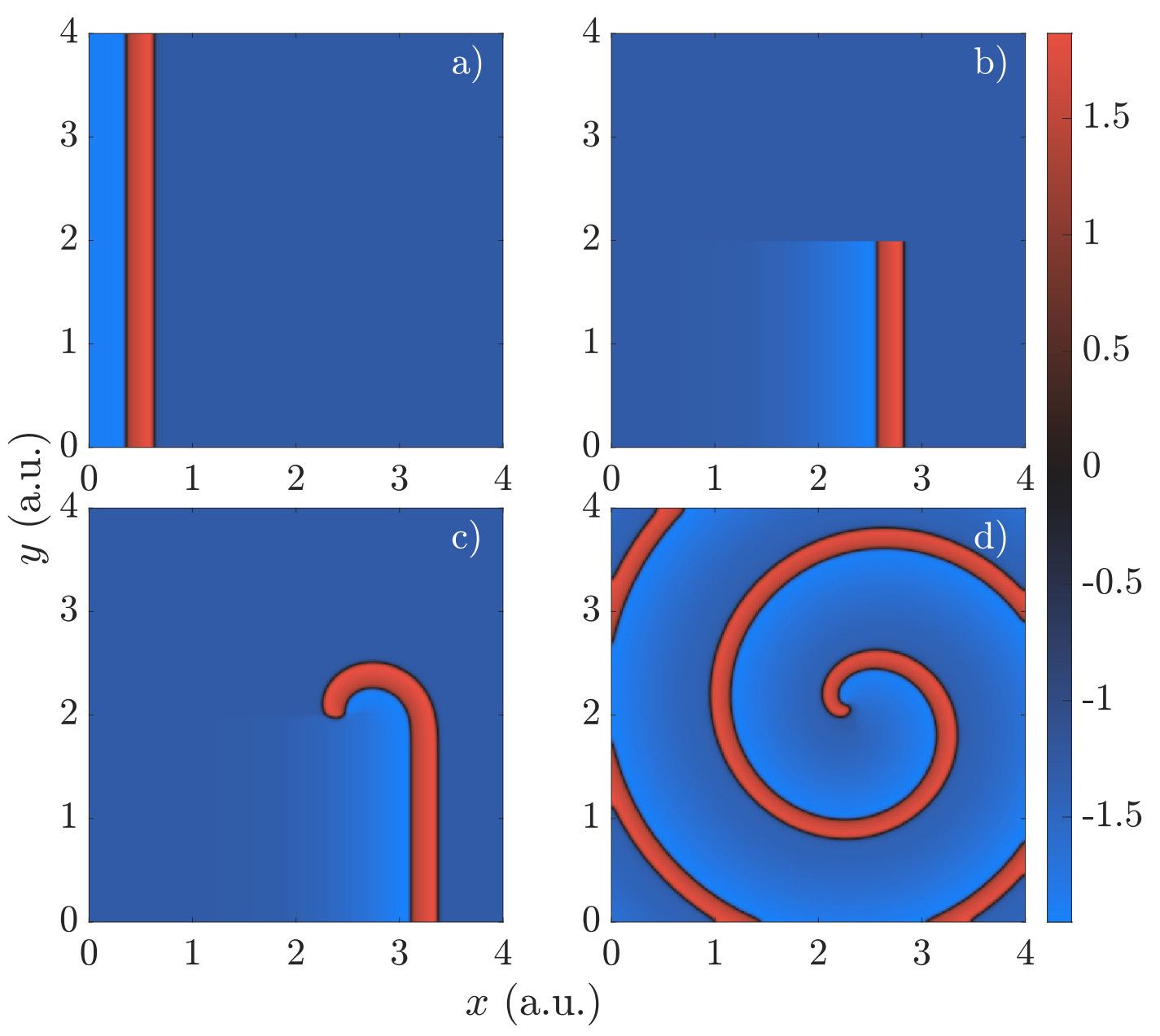

Figure 1.17: Spiral wave formation shown in the variable $u$ in a two-dimensional FitzHugh-Nagumo model, as described by Eqs. 1.3. Parameters used: $a=1, b=0.5, \nu=0.02, D_{u}=10^{-2}$, for $N=400$ points at space discretization of $d x=10^{-2}$. Integrated with explicit Euler method for a time of $T=50$. a) shows a planar wave traveling through the system, which was initiated by perturbing a strip of width 0.1 on the left boundary from the steady state. All points were set to the stable state at $\left(u_{s}, v_{s}\right)=(-1.2879,-0.57582)$, except for the excited strip that was set to $\left(u_{s}, v_{s}\right)+(1.5,0)$. b) shows the planar wave being cut off in the middle. This was done by setting half of the system to the steady state after the wave had passed about half of the domain. In c) the start of spiral formation is shown. The end of the cut-off planar wave has started to move backwards and curl up unto itself. d) shows a fully developed spiral wave at the end of the integration time. Colormap is from [136]. 


\subsection{Modeling patterns in $D$. discoideum}

The mechanism of cell aggregation in $D$. discoideum has been studied since the beginning of the 20th century $[21,145,146]$. The idea of signal relay via excreted chemicals was proposed in 1942 by Runyon, who observed that streams of cells divided by a membrane would line up regardless of the inhibition of direct contact $[21,147]$. The experiments of Bonner in 1947 confirmed this, and showed that a fluid flow could lead to triangular aggregation territories in $D$. discoideum cells [148]. The chemical used for signal relay was initially called "Acrasin", a general term given to substances used in chemotaxis [21]. Later in 1968, the specific chemical that D. discoideum employs for pattern formation, cAMP, was identified [100].

After the discovery of these basic components of pattern formation in D. discoideum, it took only three years until the first attempts to model the system were made [149]. Considering that the knowledge on the dynamics of the different steps in the molecular cascade was limited, it is not surprising that one of the first models was based on a top-down approach, inspired by timescales found experimentally. However, a bottom-up approach to understanding pattern formation in D. discoideum was proposed around the same time in a reaction-diffusion model [150]. While reaction-diffusion equations were more closely related to the molecular dynamics the cells exhibit, eleven years passed until a study could reproduce wave relay and spiral formation in such a model [151].

During the 1970s and 80s the molecular basis for pattern formation was studied in a number of groups. This led to important clarifications of the specific assumptions and rates a model could use. Among those are fundamental discoveries such as the receptor inactivation as a mechanism to induce a refractory period [152] and the activation of ACA via the same receptors [153]. More specifically, many rate constants and time scales of processes in the signaling cascade were determined as well $[152,154,155]$.

\subsubsection{Martiel-Goldbeter model}

Based on the wealth of experimental data collected in the 1970s and 80s, Martiel and Goldbeter suggested a model based on the rate constants of the processes known in the signaling cascade [156]. The authors started at initially ten kinetic rate equations representing the molecular processes described in sec. 1.2.2. Based on the assumption that binding reactions happened much more rapidly than conformational changes, the authors reduced the system to four equations. A further simplification involving the assumption of a constant intracellular ATP concentration allowed a reduction to a three variable system. An extensive derivation of the model from the initial kinetic equations can be found in appendix $\mathrm{A}$ of [156]. A de-dimensionalized version of the 
three-component Martiel-Goldbeter model (MG-model) is shown here:

$$
\begin{aligned}
\partial_{t} \rho & =k_{1}\left[-f_{1}(\gamma) \rho+f_{2}(\gamma)(1-\rho)\right] \\
\partial_{t} \beta & =q \sigma \alpha \Phi(\rho, \gamma) /(1+\alpha)-\left(k_{i}+k_{t}\right) \beta \\
\partial_{t} \gamma & =D \nabla^{2} \gamma-v(y) \cdot \nabla \gamma+k_{t} \beta / h-k_{e} \gamma, \\
\text { with } \quad f_{1}(\gamma) & =\frac{1+\kappa \gamma}{1+\gamma}, \quad f_{2}(\gamma)=\frac{\mathcal{L}_{1}+\kappa \mathcal{L}_{2} c \gamma}{1+c \gamma}, \\
\Phi(\rho, \gamma) & =\frac{\lambda_{1}+Y^{2}}{\lambda_{2}+Y^{2}}, \quad Y(\gamma, \rho)=\frac{\rho \gamma}{1+\gamma}
\end{aligned}
$$

In this version of the model, the system state is determined by three variables: the active receptor fraction $\rho$, the amount of intracellular cAMP $\beta$ and the amount of extracellular cAMP $\gamma$. The terms in $\partial_{t} \rho$ represent the switching of active receptors to the inactive state and vice versa, both based on the pool of active or inactive receptors and a nonlinear activation function $f_{1} / f_{2}$, as shown in Fig. 1.18 and Eq. 1.4. Intracellular cAMP $\beta$ increases at the nonlinear production rate $\Phi$ dependent on the amount of extracellular cAMP and the active receptor fraction, see Eq. 1.5. In addition, $\beta$ can be degraded internally at a rate $k_{i}$ and transported to the extracellular medium at a rate of $k_{t}$, see Fig. 1.18. Finally, changes in the extracellular cAMP concentration $\left(\partial_{t} \gamma\right)$ are due to a number of causes: diffusion with the diffusion constant $D$, advection based on a flow velocity $v(y)$, transport from within the cells, and extracellular degradation at the rate $k_{e}$, see Eq. 1.6.

In the production term in $\partial_{t} \beta$ there are a number of factors. The parameter $q$ describes a relation in binding affinity between cAR receptor activation and the subsequent cAMP production. It is based on the disassociation constant of the cAMP-receptor complex in the active state $K_{R}$ and the Michaelis-Menten constant of ACA $K_{M}$ as $q=K_{M} / K_{R}$. An ACA activity factor is constructed from $K_{M}$ and the maximum activity of ACA $V_{M}$ in $\sigma=V_{M} / K_{M}$. The final parameter in this term is the ATP concentration inside the cells $\alpha$. In $\partial_{t} \gamma$ there are some geometrical factors, that being the gradient operator $\nabla=\left(\partial_{x}, \partial_{y}\right)$, the diffusion operator $\nabla^{2}=\partial_{x}^{2}+\partial_{y}^{2}$ and the ratio of extra- to intracellular volume $h$. The factor of $k_{1}$ in $\partial_{t} \rho$ corresponds to the desensitization rate of active cAMP receptors. Since $k_{1}<1$ this slows down the dynamics of $\rho$ in contrast to $\beta$ and leads to the timescale separation described in section 1.4.

There are some parameters left to define in the function definitions. This includes $\kappa$ in $f_{1}$, a relative timescale of the two desensitization rates $k_{1}$ and $k_{2}$ of cAR 1 where the first describes the conformational change without and the latter with a bound ligand as $\kappa=k_{2} / k_{1}$. Similar timescales were defined by taking ratios of the re- to the desensitization steps for the case of cAR1 without bound $\operatorname{cAMP}\left(L_{1}=k_{-1} / k_{1}\right)$ and the case of cAR1 with bound cAMP $\left(L_{2}=k_{-2} / k_{2}\right)$. Additionally, a relative concentration scale of disassociation is defined via $K_{R}$, the constant for the active receptor state, and $K_{D}$, its equivalent in the inactive case as $c=K_{R} / K_{D}$. The last two parameters in the model $\lambda_{1}, \lambda_{2}$ are combinations of nondimensional relations in $\lambda_{1}=\lambda \theta / \epsilon$ and $\lambda_{2}=1+\alpha \theta /(\epsilon+\epsilon \alpha)$. In these two equations, three more de-dimensionalized rates were introduced as $\lambda, \theta$ and $\epsilon$. These are connected to the dynamics of ACA. $\theta$ being the ratio 


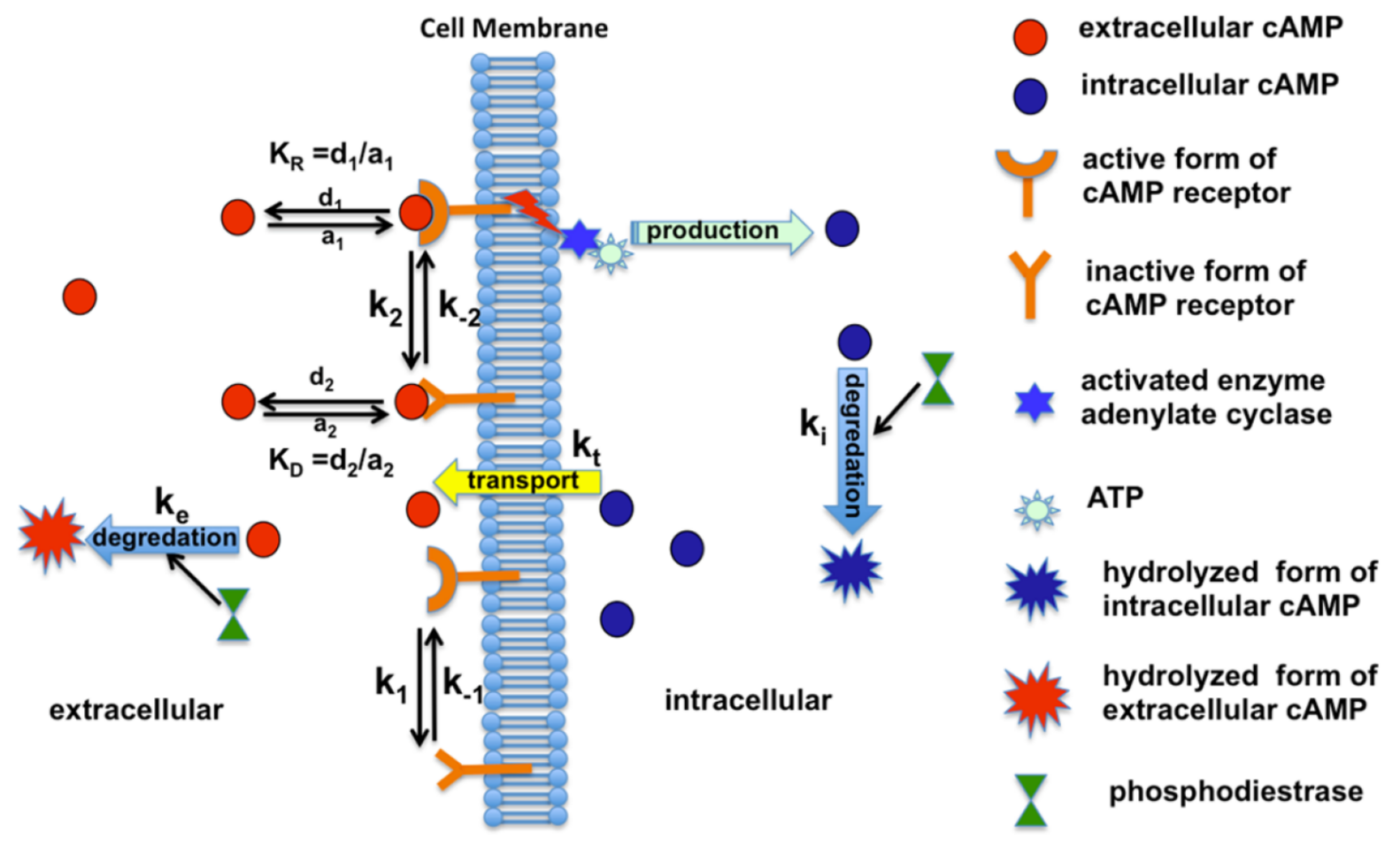

Figure 1.18: Sketch showing the chemicals and reactions in the MG-model. Extracellular cAMP binds to the active form of the cAMP receptor, triggering an intracellular signal cascade, which leads to the intracellular production of cAMP. Intracellular cAMP is degraded by PDE or transported to the extracellular medium. After cAMP binding, the receptor switches to the inactive state, which is less likely to bind cAMP. The receptor switches back to the active state, if the extracellular cAMP concentration is low enough. To facilitate the decrease of this concentration, the cells produce and release a PDE that degrades extracellular cAMP. Reproduced with minor modifications Eckstein T, Vidal-Henriquez E, Bae A, Zykov V, Bodenschatz E and Gholami A. (2018) Influence of fast advective flows on pattern formation of Dictyostelium discoideum. PLOS ONE. Licensed under Creative Commons Attribution 4.0 (CC BY). [157]

the of Michaelis constant for the ACA-receptor complex to the Michaelis constant for the free form of ACA. Similarly, $\lambda$ is the ratio of the catalytic constants of the unbound and bound state of ACA. Finally, $\epsilon$ is the coupling constant for the activation of ACA by the cAMP-receptor-ligand complex.

The values considered were mostly taken from the original paper [156, table I,II], as these were based on experimental observations. Notably, the parameters $\sigma$ and $k_{e}$ were chosen to explore the parameter space of the system, considering that they represent cAMP production and degradation. We changed two parameters to recreate the decrease in cAMP production as an effect of caffeine, see chapter 4. Those being the Michaelis- 
Menten constant for the binding of ACA to ATP $K_{M}$ and the affinity constant for the activation of ACA by the cAR1-cAMP complex $\epsilon$. As the production of cAMP is reduced under the effect of caffeine, we emulated its effect by respectively increasing $K_{M}$ or decreasing $\epsilon$. Moreover, we included advection in the model, to describe our experiments with $D$. discoideum in microfluidic channels under flow, see chapters 2 and 3. To understand our experiments with $\mathrm{pdsA}^{-}$cells, we drastically reduced the extracellular degradation rate $k_{e}$, see chapter 3 .

The system can be simplified one last time by assuming that internally produced cAMP is immediately transported outside of the cells in the medium. This corresponds to assuming a fast dynamics for $\beta$, allowing for adiabatic elimination of $\beta$. Defining a composite variable $s$ as $s=q \sigma \alpha k_{t} /\left[h(1+\alpha)\left(k_{i}+k_{t}\right)\right]$ the equations then read as follows:

$$
\begin{aligned}
& \partial_{t} \rho=k_{1}\left[-f_{1}(\gamma) \rho+f_{2}(\gamma)(1-\rho)\right] \\
& \partial_{t} \gamma=D \nabla^{2} \gamma-v(y) \cdot \nabla \gamma+s \Phi(\rho, \gamma)-k_{e} \gamma
\end{aligned}
$$

Note that the model presented here includes terms for diffusion and advection of the extracellular cAMP. The other fields are stationary, as they are coupled to the cells. The extension to include diffusion was proposed by Tyson in 1989 and allowed him to show spiral formation in the model [158]. Consequently, a version of the MG model modified to include the G-protein signaling dynamics was introduced in 1998 [159]. In 2015, Gholami et al suggested a further extension by including advective flow [160], which allowed the description of how a flow impacts the pattern formation in D. discoideum. The inclusion of advection in systems showing Turing patterns strongly influences the pattern formation, as was shown in the BZ-reaction [161,162]. For a systematic theoretical treatment of reaction-diffusion dynamics under the influence of flow and geometrical constraints see Van Gorder et al [163].

\subsubsection{Wave creation in $D$. discoideum models}

How to introduce waves at a regular period in models of the pattern formation in $D$. discoideum has been a long standing question. This is especially relevant, since the system can sustain target centers. As was mentioned in section 1.4, these patterns require a constant wave production mechanism. Considering that a purely homogeneous excitable system cannot repeatedly produce waves, the idea of introducing inhomogeneities was born. The idea of special cells, called "pacemaker" cells, that determine where aggregation areas are centered was proposed quite early in the history of $D$. discoideum modeling [164,165]. The concept of pacemaker cells spread quickly through the research community at the time and was mostly accepted [164]. Though a considerable amount of research was performed to find and describe these cells, it was not successful. Even the rather recent book by Kessin still mentions this mystery: "All that we know of pacemaker cells is that they are first among equals and are not genetically distinct." [21, p. 18]. In 2010 the existence of pacemaker cells was seriously questioned by direct light microscopy experiments [113]. These showed an area of cells to be the wave center and single cells moving in and out of this area, thus proving that the center was an emergent property and not defined by single special cells. The same was found in a study 
that visualized cAMP directly via fluorescent probes [166]. There a synchronized pulse of 13 cells in a $100 \times 100 \mu \mathrm{m}^{2}$ region was shown, indicating that the wave initiation event included a number of cells. However, a direct study of the excitability of single cells has shown differences in both the excitability threshold and the intensity of the response [167]. Consequently, the wave centers probably are not defined by single special cells but rather are an emergent property dependent on the excitability of cells in a region.

A more biological approach was proposed by Goldbeter et al in 1997 [168]. Instead of assuming that some special cells were inherently oscillatory, the authors introduced a developmental path model. The cell state in this model is described by the local cAMP production and degradation, which corresponds to ACA and PDE activity respectively. These parameters were then increased according to a path through phase space, chosen according to experimental observations of the enzymes' activities during aggregation. This empirical path through phase space could reproduce known patterns. Together with a slight initial desynchronization of the cells along this path, this model can explain spiral formation in a more natural way. In this model, cells that are advanced along the developmental path reach the oscillatory region first and then act as pacemakers.

Another way of destabilizing an excitable system is through its boundary conditions. It has been shown in reaction diffusion systems that a zero or Dirichlet boundary condition can destabilize the steady state and lead to pattern formation at parameters that would be stable with zero flux or Neumann boundary conditions [169,170]. In a more recent work, the induction of periodic wave pulses by Dirichlet boundary conditions was shown in a reaction-diffusion system [171]. Recently, a similar mechanism was applied in the MG model [172]. In this case a combination of a Dirichlet boundary condition and advective flow led to wave creation. We studied this wave formation mechanism experimentally in a microfluidic channel with $\mathrm{pdsA}^{-}$cells, which are normally stable, see chapter 3.

Finally, a natural way of introducing waves was proposed by Vidal and Gholami in 2019 [173]. This wave creation mechanism is based on local inhomogeneities of the cell density. To model a spatially inhomogeneous cell density, the model was transferred to a cell automaton model of the MG type. Crucially, this allows to define space points without cells present that differ in behavior from occupied spaces. Assuming that PDE is produced locally but diffuses, the empty spaces can degrade cAMP but not produce it. With this approach, the authors found spontaneous center formation for sub monolayer densities. We have used this mechanism in experiments with non-excitable obstacles and cell densities below confluency as described in chapter 4 .

\subsubsection{Other models for pattern formation in $D$. discoideum}

Considering that pattern formation in $D$. discoideum held much scientific interest in the second half of the 20th century, it is not surprising that more researchers proposed models to explain it. Among these is the first model to understand D. discoideum aggregation as an instability, proposed by Keller and Segel [150], whose equations are 
given by:

$$
\begin{aligned}
& \frac{\partial a}{\partial t}=-\nabla \cdot\left(D_{1} \nabla \rho\right)+\nabla \cdot\left(D_{2} \nabla a\right) \\
& \frac{\partial \rho}{\partial t}=-k_{1} \rho \eta+k_{-1} c+a f(\rho)+D_{\rho} \nabla^{2} \rho \\
& \frac{\partial c}{\partial t}=k_{1} \rho \eta-\left(k_{-1}+k_{2}\right) c+D_{c} \nabla^{2} c \\
& \frac{\partial \eta}{\partial t}=-k_{1} \rho \eta+\left(k_{-1}+k_{2}\right) c+a g(\rho, \eta)+D_{\eta} \nabla^{2} \eta
\end{aligned}
$$

This model describes pattern formation by means of four fields. The concentration of cells per area $a$, the concentration of the chemoattractant $\rho$ and the concentration of the degradation agent of this attractant $\eta$. The final field corresponds to the chemoattractant-degradation complex $c$. Changes in $a$ are due to the cells' chemotactic movement, which depends on the relative chemoattractant and cell concentration, see Eq. 1.7. The chemoattractant field $\rho$ changes because of binding and unbinding to its degradation agent at rates $k_{1}, k_{-1}$ respectively, see Eq. 1.8. Additionally, the cells produce the chemical at a rate $f(\rho)$ and it diffuses with diffusion constant $D_{\rho}$. The enzyme-ligand complex $c$ has a source term in the binding of the chemical to the ligand at rate $k_{1}$, see Eq. 1.9. Then the complex is dissolved either by disassociation or by degradation at rates $k_{-1}, k_{2}$ respectively. Additionally, the complex diffuses with diffusion rate $D_{c}$. Finally, the degrading agent $\eta$ has the same steps of forming the complex $c$ at rate $k_{1}$ and being released from the complex at rates $k_{-1}, k_{2}$, see Eq. 1.10. Moreover, the cells produce the agent at rate $g(\rho, \eta)$ and it diffuses with diffusion constant $D_{\eta}$. Note that the in the equations both the gradient operator $\nabla=\left(\partial_{x}, \partial_{y}\right)$ and the diffusion operator $\nabla^{2}=\partial_{x}^{2}+\partial_{y}^{2}$ were used. This model was later extended by Barchilon and Segel to include a more detailed description of a mechanism allowing for adaptation to cAMP, which had been found experimentally [174].

In contrast to these very detailed models, Kessler and Levine suggested a radically reduced model, aiming to find the minimal prerequisites for the phenomenona seen in D. discoideum pattern formation [175]. This cellular automaton model used empirical rules for the production and relay of cAMP as a simplification of the molecular specifics. With the addition of movement rules, both spiral wave formation and cell streaming could be reproduced. This model only uses a single field in the cAMP concentration $\gamma$ (variables were renamed to the according names in the MG model presented above):

$$
\frac{\partial \gamma}{\partial t}=D \nabla^{2} \gamma-k_{e} \gamma+(\text { sources })
$$

Here $D$ is the diffusion constant that was set to the lattice size squared and $\nabla^{2}=\partial_{x}^{2}+\partial_{y}^{2}$ the diffusion operator. The degradation term includes a constant and uniform PDE concentration in the degradation rate $k_{e}$. Finally, the sources term describes the addition of cAMP to the system through release by the cells. The release rules are modeled after the excitable characteristics of the system. Each bion initially is in the excitable state 0. If the cAMP concentration at the bion is above a threshold concentration $\gamma>\gamma_{T}$, it 
Chapter 1 - Introduction

enters the excited state 1 . In this state a certain amount of cAMP $\Delta \gamma$ is released by the bion over a time $\tau$. After this excited period is over, the cell enters the refractory state 2 in which it is not excitable. The bion reverts to state 1 after a refractory period $t_{R}$. In addition to these rules for the release and degradation of cAMP, motility rules were introduced. Each bion in state 1 moves if it detects a gradient above a motion threshold of $\gamma_{T}^{\prime} / D$ and the next grid point in that direction is unoccupied. 


\subsection{Methods}

To allow for the reproduction of the results shown in this dissertation, the methods used in this thesis shall be laid out in the following. In this description we start at the handling and culturing of $D$. discoideum cells, continue with the experimental setup and image acquisition and finish with image processing and analysis.

\subsubsection{Cell culture}

D. discoideum cells of the Ax2 and pdsA- strains described in sec. 1.2.4 were kept in a $-80^{\circ}$ freezer as spores or as cells until needed for experiments. Before usage, the frozen samples were allowed to thaw at room temperature. After this, the cell solution was mixed within in the ampulla and then suspended in $10 \mathrm{ml}$ HL-5 medium (a growth medium for axenic cells, see below) and plated in a petri dish. The cells were incubated at room temperature to allow for normal growth. Note that all cell culture steps were performed under a sterile hood, to prevent contamination of the cell culture by bacteria as these would represent an uncontrolled food source for the amoebae.

For multiple experiments with the same cell culture, single strain populations were split into parts and cultured up to 12 times. This entails removing the old HL-5 medium from a confluent petri dish. Consequently, the amoebae could be dislodged by repeatedly pipetting $10 \mathrm{ml}$ fresh HL-5 onto the dish. Based on cell growth, different amounts of cell solution were mixed with $10 \mathrm{ml} \mathrm{HL}-5$ and plated in fresh petri dishes. For usage on the next day, $2.5 \mathrm{ml}$ cell solution was necessary. $0.5 \mathrm{ml}$ were sufficient, with two days of growth. If the cells were allowed to grow for three days only 1-2 drops of solution was needed.

To prepare cells for an experiment, they were detached from the petri dish using 10 ml Phosphate buffer ( $\mathrm{Pb}$, a non-nutrient medium for the cells, see below). The cells in solution were then pelleted by centrifugation (3 minutes at $1000 \mathrm{rpm}$ ) and resuspended in fresh $\mathrm{Pb}$. This washing step was repeated a second time to guarantee that no growth medium remained in the solution. After washing, the cells were suspended in $10 \mathrm{ml} \mathrm{Pb}$ with or without caffeine. This solution was incubated in an Erlenmeyer flask on a shaker (at $150 \mathrm{rpm}$ ). The cells were starved until the beginning of the experiment. Note that initial starvation time plays an important role in pattern formation [176].

After sufficient cell starvation, the cells were washed in $\mathrm{Pb}$ again, as described above. Depending on the experiment, the cells were then dissolved in: $0.4 \mathrm{ml} \mathrm{Pb}$ for microfluidic experiments; 10 or $20 \mathrm{ml} \mathrm{Pb}$ for petri dish experiments. The caffeine concentration of the buffer was again adjusted to the one applied during starvation. To exclude density dependent phenomena, the cell density was monitored [109,113,177]. For this, the cell solution was diluted 1:200 or 1:10 for channel or petri dish experiments, respectively. The density in the diluted solution was then determined using a hemocytometer (Neubauer Zählkammer). Cell densities above the target of $\rho_{\text {channel }}=5 \times 10^{7}$ cells $/ \mathrm{ml}$ or $\rho_{\text {petri dish }}=$ $2 \times 10^{6}$ cells $/ \mathrm{ml}$ were diluted accordingly. These two densities resulted in area densities of the cells of $\rho_{\text {channel }}^{\prime}=5 \times 10^{3}$ cells $/ \mathrm{mm}^{2}$ and $\rho_{\text {petri dish }}^{\prime} \approx 9.1 \times 10^{3}$ cells $/ \mathrm{mm}^{2}(0.5$ mono-layer and $\sim 0.9$ mono-layer based on a cell size of $10 \mu \mathrm{m} \times 10 \mu \mathrm{m}$ ). 


\section{Buffers}

HL-5 is a traditional growth medium for axenic D. discoideum strains that was developed to contain as few ingredients as possible [178]. HL-5 was prepared from a HL-5 powder (HL5 Medium including Glucose - Formedium Ltd, Norfolk, England). The powder was dissolved in double-distilled water at a rate of 35.5 gr powder per liter. Then the medium was autoclaved and filtered before storage.

Phosphate buffer $(\mathrm{Pb})$ is a non-nutrient buffer we used to starve the cells, while still supplying them with ions. It consists of double-distilled water, in which 2 gr of $\mathrm{KH}_{2} \mathrm{PO}_{4}$ and 0.36 gr of $\mathrm{Na}_{2} \mathrm{HPO}_{4} \times 2 \mathrm{H}_{2} \mathrm{O}$ are dissolved per liter. The $\mathrm{pH}$ value of the buffer was adjusted to 6.0 by adding either $\mathrm{NaOH}$ or phosphoric acid.

\subsubsection{Experimental setup}

After the cell handling has been discussed above, we now briefly describe the experimental systems used to study the cells. This includes not only the dark-field microscopy setups used but also the actual environment the cells were imaged in.

\section{Dark-field microscopy}

The idea of a dark-field experiment is to visualize samples using their light scattering. To allow for this, the sample is illuminated with a ring light source, see Fig. 1.19c). The main light beam is focused such that its image is in focus right outside of the camera objective. In this configuration only scattered light can reach the camera.

We utilized two dark-field microscopes at low magnifications, shown in Fig. 1.19. Technically, the first setup used is a pseudo-dark-field setup, see Fig. 1.19a). This setup consists of ring light source (Advanced Illumination, Rochester, VT, USA) below which the sample is mounted. Image capture is done using a monochrome 12-bit CCD camera (QIClick-F-M-12, QImaging, Surrey, Canada) equipped with a telecentric lens of magnification 1.6x (0.16X SilverTL Telecentric Lens, Edmund Optics Inc, Barrington, NJ, USA) and operated using an image capture software (Micro-Manager [179]).

The second setup has a slightly different configuration, shown in Fig. 1.19b). It uses a green LED ring as a light source (LED Miniatur Ringbeleuchtung LSR24-G from LUMIMAX). The light is spread out by a 7 inch focal length fresnel lens (11.0" x 11.0", 7" Focal Length from Edmund Optics, bottom side in-house coated with an anti-reflective coating) to illuminate the sample. The image acquisition system uses a monochrome 12bit CCD camera (QIClick-F-M-12, QImaging, Surrey, Canada) and a $50 \mathrm{~mm}$ focal length objective (MVL50M23 from Thor Labs) again operated using the same image capture software (Micro-Manager [179]). The difference between our pseudo-dark-field setup and a normal dark-field setup is that we did not use a lens to diffuse the illumination from the ring light. Additionally, both setups use commercial hair dryers, which were modified by taking nearly all heating units out of the circuit, to slightly warm the top of the sample. This was done to avoid condensation on the lid of the petri dishes. 

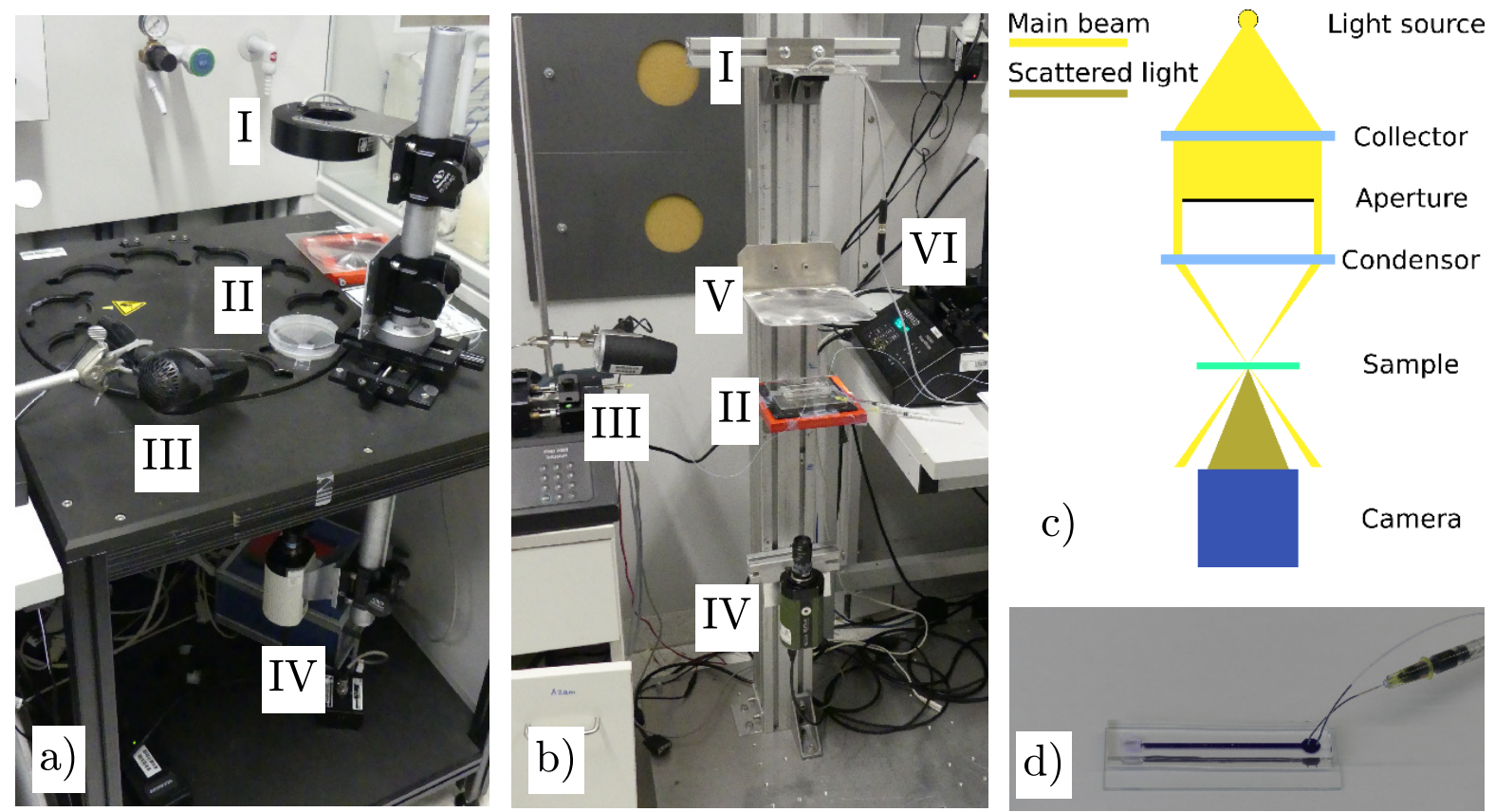

Figure 1.19: Dark-field microscopy setups used in this thesis. a) shows the pseudo-darkfield setup consisting of a QClick camera, a telecentric lens and a white ring light. In b) the second dark-field setup is shown. This setup uses the same camera as the one shown in a) but a $50 \mathrm{~mm}$ focal length objective, a green LED ring light and a fresnel lens. I denotes the light source, II the sample, III the fan, IV the camera, V the fresnel lens and VI a syringe pump. c) shows the light path of a dark-field microscope. d) shows a microfluidic channel as is seen in b) at II in larger magnification.

\section{Microfluidic fabrication}

Most of the environments, in which we study the cells, are custom made from polydimethylsiloxane (PDMS), a flexible, transparent polymer that allows rapid setup production. PDMS is used since it is biocompatible and allows for gas diffusion, meaning that cells are supplied with oxygen. For these reasons, PDMS is commonly employed in the life sciences [180]. PDMS setup production is based on soft lithography [180]. The polymer consists of two ingredients: the polymer base and the corresponding crosslinker (Sylgard 184, Dow Corning GmbH, Wiesbaden, Germany). These components are mixed (at a 1:10 ratio) and degassed. Then the liquid is cast in a mould and degassed again. Finally, the mixture is baked at $75^{\circ} \mathrm{C}$ for one hour.

We produced microfluidic channels by standard soft lithography methods [180]. To produce a casting mould via photolithography, we first spin coated a silicon wafer with a thin (about $100 \mu \mathrm{m}$ ) layer of photoresist (SU-8 100, Micro Resist Technology GmbH, Berlin, Germany). This wafer was then baked and exposed to UV light using a mask aligner and a film mask to transfer the features of the mask to the photoresist (custom design, produced by Micro Lithography Services Limited, Essex, UK). After exposure, 
the unexposed photoresist was washed away using a developer agent (mr-Dev 600, Micro Resist Technology GmbH, Berlin, Germany). Using these moulds, channels were cast in PDMS as described above. Then single channels were cut out of the PDMS block using a scalpel. Holes were punched through the PDMS to allow cell loading, liquid withdrawal and serve as a fluid reservoir, using hole punchers (Harris Uni-Core-7.00 and Harris UniCore-0.75, Sigma-Aldrich, St. Louis, MO, USA). Then the PDMS was bonded with a glass slide (76x26 mm, VWR, Darmstadt, Germany). For this, we activated the surface of both PDMS block and glass slide via 25 s exposure to an air-plasma (PDC 002, Harrick Plasma, Ithaca, USA). An example of a straight channel is shown in Fig. 1.20a).

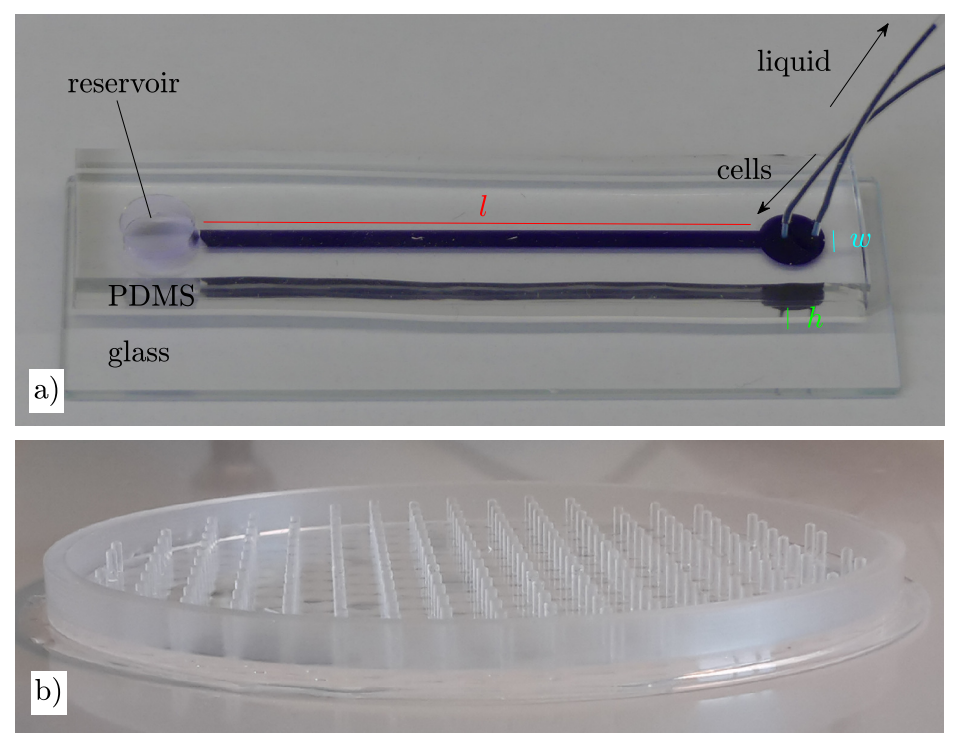

Figure 1.20: Examples for the PDMS setups used in this thesis. a) shows a microfluidic channel. It was tinted purple with crystal violet ink to increase visibility. On the left the reservoir is seen as a circle with $7 \mathrm{~mm}$ diameter. On the right, two smaller holes of diameter $0.75 \mathrm{~mm}$ with tubing are shown, one is used for cell loading, the other to draw liquid through the channel. The channel has a width of $w=2 \mathrm{~mm}$, a height of $h=100 \mu \mathrm{m}$ and a length of about $l=4 \mathrm{~cm}$. In b) a petri dish setup is shown. The entire flat surface has a diameter of $7.5 \mathrm{~cm}$, the obstacles themselves have a height of $1.3 \mathrm{~mm}$ and a diameter of $1 \mathrm{~mm}$. The dish is encased by a rim of height $5 \mathrm{~mm}$ to hold the liquid inside.

After the cells had been prepared according to the methods described in section 1.6.1, they were introduced into the experimental setup. The microfluidic channel was connected to a syringe pump using tubing. Consequently, the cell solution was rigorously vortexed to avoid cell clumping, as local cell density changes can induce target centers, see chapter 4, Fig. S5. Then the cells were inserted into the channel using a syringe and a piece of tubing connected to the channel. Afterwards, the cells were allowed to settle down for $10 \mathrm{~min}$. To ensure a zero cAMP boundary condition at the upstream part of the channel, cells were removed from the reservoir and it was filled with fresh 
$\mathrm{Pb}$. Finally, a flow was applied to the channel using a syringe pump that was connected via tubing (PHD 2000 Infuse/Withdraw Syringe Pump from Harvard Apparatus, USA, used with gasstight glass syringes from Hamilton, USA).

The setups for the petri dish experiments were produced using moulds, with features on the order of millimeters and not micrometers. We used many different moulds, some of which were produced by photolithography. Most of them were created by first milling down a slab of Polymethyl methacrylate (PMMA), then drilling indentations into the surface and finally sanding down roughnesses. In this way, we created moulds containing a smooth surface with cylindrical obstacles (normally 1-3 mm height, $1 \mathrm{~mm}$ diameter), such as the one shown in Fig. 1.20b). Before using the petri dish PDMS setups they were activated by brief (25 s) exposure to an air plasma. This made the PDMS surface hydrophilic and thus avoided the formation of air bubbles when the cell solution was poured in. Cells were prepared as described in section 1.6.1. The cell solution was vortexed intensely to break up possible cell clumps and the liquid poured into the PDMS dish.

\subsubsection{Image processing}

Most of the experimental data collected was in the form of videos showing the pattern formation of $D$. discoideum using the dark-field microscopes described in sec. 1.6.2. Thus a range of image processing techniques were used to i) reduce the noise level and ii) quantify the cAMP wave patterns. An overview of the workflow is shown in Fig. 1.21 .

As the original videos suffer from unequal illumination and long term intensity drift, we normalize the image stacks on a pixel-per-pixel basis. To do this, a 6th order polynomial is fitted to the intensity range of each pixel as a function of time. This polynomial describes the long term drift and constant background illumination at the pixel. See Fig. 1.21a) for an example of a polynomial fit to the intensity of one pixel over time. After subtracting this background signal, the values are centered around zero, as can be seen in the normalized version of the signal in Fig. 1.21b). This normalization greatly enhances contrast by equilibration of illumination, as can be seen by comparing the snapshots in Fig. 1.21c) and d).

While the waves are more easily visible in the normalized videos, the normalization technique does introduce more noise into the system, since it is purely pixel based. Therefore, we smooth the images by performing a two-dimensional gaussian bandpass filter. This filtering process works by first applying the two-dimensional fast-Fouriertransform (FFT) to the images and then filtering in the frequency domain. The FFT representation of the images is multiplied with a two-dimensional gaussian bandpass filter $B P$ as defined by the multiplication of a gaussian low-pass filter $L P$ and a gaussian high-pass filter $H P$ : 

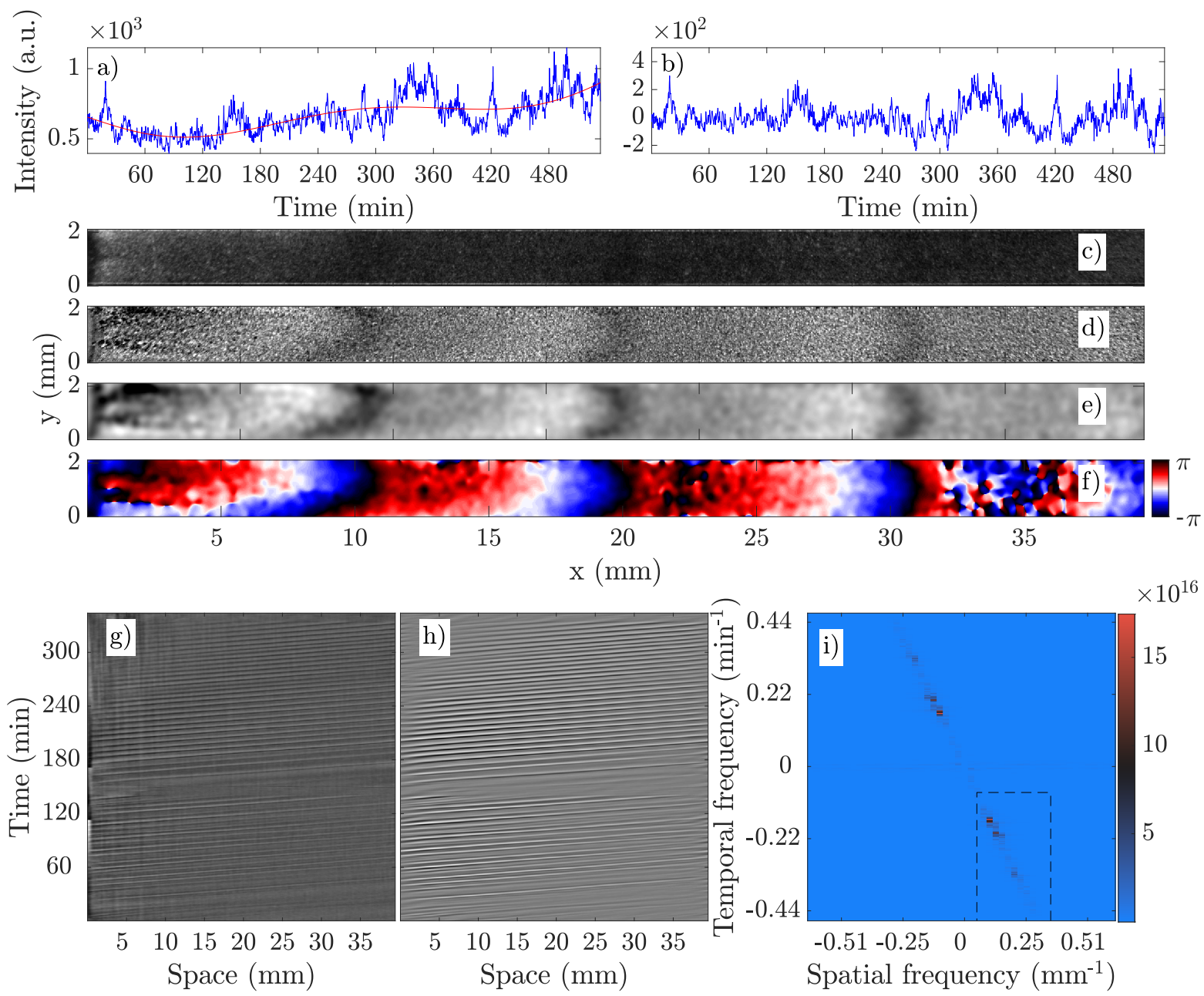

Figure 1.21: Overview of the workflow that was used for image analysis, showing examples of a channel experiment with pdsA ${ }^{-}$cells at a flow velocity of $V_{f}=1.5$ $\mathrm{mm} / \mathrm{min}$. a) shows the original signal of an arbitrary pixel close to the inlet in blue and the 6th order polynomial fitted to it in red. After subtraction the signal is centered around zero, as shown in b). c) shows an original snapshot of the full channel with waves. d) shows the same timepoint in the normalized image, with much clearer waves. In e) the same frame is shown after bandpass filtering, which has a considerably more smooth intensity distribution. f) finally shows the instantaneous phase extracted from the smoothed image stack. Averaging the pixels of e) along the $y$ axis and stacking them leads to a space time plot, shown in g). These plots show traveling waves as lines and are filtered by frequency selection. A filtered version of the same plot is shown in $\mathrm{h}$ ). The selection is depicted the power spectrum of the plot in i). All frequencies except those inside the black rectangle are set to zero. The resulting frequencies are transformed back to real space. Colormap is from [136]. 


$$
\begin{aligned}
& L P(r)=\exp \left[-r^{2} / \sigma_{l}^{2}\right] \\
& H P(r)=1-\exp \left[-r^{2} / \sigma_{h}^{2}\right] \\
& B P(r)=L P * H P
\end{aligned}
$$

Note that since filtering was done in the FFT-domain, $r$ denotes distance to the edges of the image. Further, the threshold sizes $\epsilon_{l / h}$ ( $l$ denoting the low- and $h$ the highpass filter) have to be converted to frequencies as per $\sigma_{l / h}=L /\left(2 \epsilon_{l / h}\right)$ with the image length $L$. The values used for this filter were $\epsilon_{l}=10 \mathrm{px}(\approx 0.29 \mathrm{~mm})$ and $\epsilon_{h}=120$ px $(\approx 3.53 \mathrm{~mm})$ based on the wave thickness range reported, see section 1.2.3. After this filtering step, the images appear much smoother, as can be seen in Fig. 1.21e). For some applications, such as finding spiral centers and defining aggregation areas, we extracted the instantaneous phase from the smoothed image stacks using the Hilbert transform [181]:

$$
\tilde{x}(t)=\mathscr{H}[x(t)]=\int_{-\infty}^{\infty} \frac{x(u)}{\pi(t-u)} \mathrm{d} u
$$

Starting from a real valued signal, the Hilbert transform provides an imaginary extension of the signal such that the addition of both parts is an analytical signal [182, chapter 13]. The instantaneous phase is then calculated as the phase angle between the real and the imaginary part of the analytical signal. An example for the phase can be seen in the snapshot in Fig. 1.21f).

To compress the information shown in channel experiments, we constructed spacetime plots from the normalized videos. This is done by averaging the pixel intensity along the $y$-direction to form a one-dimensional signal and then stacking these single one-dimensional signals atop each other. A wave traveling along the channel is seen as a band of pixels darker than their surrounding pixels, followed by a bright band. This is due to the chemotactic response of the cells to cAMP gradients. When a wave washes over a cell, it initially cringes, meaning that it rounds up, thereby reducing its size. After this initial response, the cell starts elongating and moving in the direction of the positive cAMP gradient, thereby extending itself and increasing its size. During the cringe response the cells scatter relatively little light. In contrast, light scattering is increased while the cells move. Therefore, a cAMP wave passing through a cell colony results in a decrease of the scattered light followed by an increase of it. Averaging over $y$ results in an extended dark spot in the one-dimensional signal, centered at the point where the wave is darkest. As this point moves along the channel, the waves are transformed into lines in the space-time plot. An example of such a space-time plot is shown in Fig. 1.21g). As these plots show noise and unequal illumination in space, we again filter in the frequency domain. However, our approach to filtering is more direct in this case, than in the case of bandpass filtering described before. We perform the twodimensional FFT and then calculate the spectrum of the images. In this data we then remove all frequencies except for the dominating frequencies manually. The spectrum 
of the space-time plot from before and the selected frequency range (black rectangle) is shown in Fig. 1.21i). These filtered frequencies are then transformed back by the inverse two-dimensional FFT and the resulting image compared to the original data to avoid overfiltering. The resulting filtered space-time plot is shown in Fig. 1.21h).

\section{Image subtraction}

While the dark-field approach to study waves in D. discoideum is relatively straightforward, the image quality can be problematic, making the waves hard to discern, as shown above. Therefore, image processing has often been used to increase wave visibility and allow for easier analysis. A specific image enhancement technique called "image subtraction" is briefly introduced here. Slice subtraction was proposed by Siegert in 1995 to enhance the visibility of cAMP waves in D. discoideum aggregation during the mound stage [183]. Since then it has been used repeatedly in a number of studies [107,184-186]. On small time scales, the only changes in videos of $D$. discoideum aggregation should be due to the traveling waves. Therefore, subtracting images that are temporally close to each other should constitute a type of adaptive background subtraction and enhance contrast. Usually, one takes direct neighbors in the image stack or small time shifts. This means subtracting every $n$th image from the $n+1$ th (or $n+3 \mathrm{rd}$ ).

This technique is illustrated in Fig. 1.22. While the waves in the original image, as shown in Fig. 1.22a), are hard to distinguish, the ones in the subtracted image Fig. $1.22 \mathrm{~b}$ ) appear clear. To clarify the impact of image subtraction on the actual signal, a small $7 \times 7$ pixel area is averaged for each time step and then plotted in Fig. 1.22c-d) for the original and the subtracted case. As can be seen, the wave pulses are more defined after subtraction and the signal is centered around zero, corresponding to a background subtraction. However, the image subtraction is equivalent to a low-pass filter in the frequency domain of the signal, as shown in the power spectra in Fig. 1.22ef). Therefore, if the processed data shall be further analyzed, especially regarding the oscillation period, this tool has to be used with caution.

There is a second, unintended consequence of using the image subtraction technique. Larger waves consist of a dark band followed by a bright band that move through the field of view at a certain speed. The intensity returns to a medium value after the wave has passed and therefore image subtraction can introduce a second dark band. This is dependent on the wave speed, and is shown in a flow experiment in Fig. 1.23. The wave is hard to distinguish in the original snapshot in Fig. 1.23a). In comparison, Fig. 1.23b) shows a snapshot normalized as described above and Fig. 1.23c) an image subtracted version. Both image processing techniques improve the contrast in the image, allowing for easier wave recognition. While the waves are even clearer in the subtracted image in Fig. 1.23c), they also show an additional dark band, which is an artifact of subtraction. 

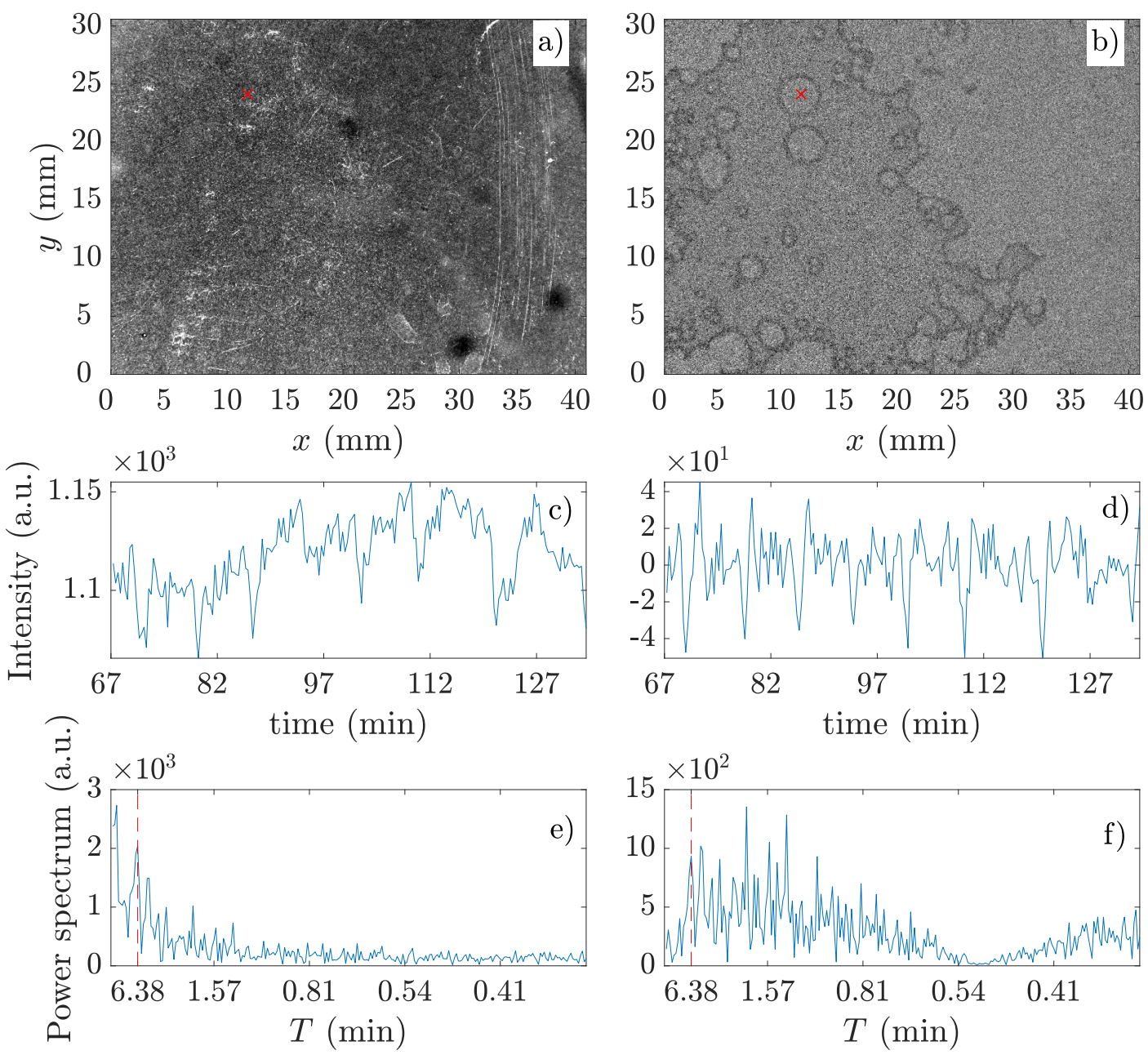

Figure 1.22: Example showing the image subtraction technique and its influence on the signal. a) shows the original dark-field snapshot of an experiment with 1 mM caffeine on $1.5 \%$ Pb-Agar. b) shows the same snapshot after image subtraction was performed on the stack, subtracting ever $n$th from the $n+3$ rd image. This corresponds to subtracting at 1 min intervals, as we capture an image every $20 \mathrm{~s}$. Note that the waves are visible in b) in contrast to in a). c-d) show a cut out of the signal over time. Signal was created by taking the average over a $7 \times 7$ pixel area of the videos centered at the red $\mathrm{x}$ in $\mathrm{a}$ ) and $\mathrm{b}$ ). Only a part of the signal is shown for clarity. Note that the pulses are clearer and the signal is centered around zero. e-f) show the corresponding power spectrum for the full signal, of which a cut is shown in c-d). The signal clearly lost its high frequency components, meaning that the subtraction method works as a low-pass filter in frequency space. The frequency corresponding to the actual waves is shown in red. 
Chapter 1 - Introduction

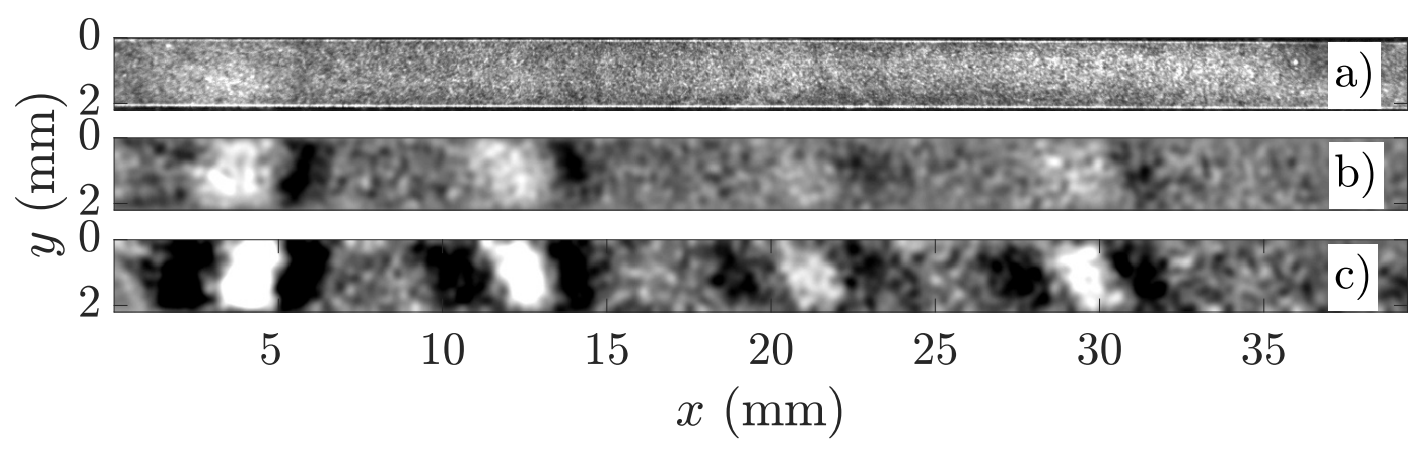

Figure 1.23: Example showing the effect of the image subtraction technique on flowinduced, fast moving waves in a flow experiment of $\mathrm{pdsA}^{-}$cells at a flow speed of $V_{f}=1.5 \mathrm{~mm} / \mathrm{min}$. a) shows the original dark-field image, in which the waves are hard to make out. In b) a normalized and bandpassfiltered version of the snapshot is shown that has clearer waves. c) shows the snapshot after image subtraction and bandpass-filtering. The waves are even clearer than in b). Note that an additional dark band in the waves was introduced as an image processing artifact. 


\subsubsection{Image analysis}

After image processing was applied to increase image quality, a number of image analysis techniques were used to extract the wave characteristics. An overview of these processes shall be given in the following, and is shown in Fig. 1.24. A snapshot of the original data is shown in its normalized and band-pass filtered state in Fig. 1.24c). Additionally, a part of the space-time plot of this experiment is shown in Fig. 1.24a), representing the spatio-temporal dynamics of the waves in one plot. For the techniques used to generate these representations from the original dark-field data, please refer to section 1.6.3.

To extract the period and the wavelength of the waves, we use the space-time plot of the signal. A cut through the plot at constant time represents a signal of the waves traveling down the channel. Conversely, a cut at a constant point in space shows the oscillations of the signal there, with multiple waves passing through. From these cuts we calculate the wave length and the period respectively via the auto-correlation $\Phi_{x x}$. This function calculates the correlation of a signal with a shifted version of itself for different shifts:

$$
\Phi_{x x}=\frac{1}{N} \sum_{n=1}^{N} x_{n} x_{n-k}
$$

The auto-correlation of a periodic signal has maxima at shifts that correspond to multiples of the periodicity [187, pg. $238 \mathrm{ff}$.]. We use this characteristic by finding the first maximum of the auto-correlation, whose distance from zero corresponds to one period. To calculate the auto-correlation we use the Wiener-Khinchin theorem [187, pg. $246 \mathrm{ff}$.] Thus, we relate $\Phi_{x x}$ to inverse Fourier transformation $\mathscr{F}^{-1}$ of the power spectral density of the signal $S_{x x}$ :

$$
\Phi_{x x}=\mathscr{F}^{-1}\left(S_{x x}\right)
$$

This measurement technique is presented for the two cuts shown in Fig. 1.24a). The signals along these cuts are plotted in Fig. 1.24g-f). The corresponding auto-correlations are shown with their respective maximum marked in Fig. 1.24h-i).

We measure the wave speed by automatically tracing the dark lines in the space-time plot. After visual inspection the points are corrected manually. This results in traces such as the one shown in Fig. 1.24a). As we assume a constant velocity, we perform linear fits of the data following a simple fit function for space $s$ vs time $t: s(t)=v_{\text {wave }} \times t+b$ to extract the wave velocity $v_{\text {wave }}$, as shown in Fig. 1.24b).

To measure the decay length of the waves $l_{d}$ we start with the same wave traces as described above. Then we read out the signal intensity along these traces and normalize the values by the maximum intensity. The decay length is then measured as the length between the first point of the trace and the first point at which the signal drops below $10 \%$ of its maximum value, as shown in Fig. 1.24e).

For measuring the thickness of the waves, we slice through snapshots of the waves (normalized and band-pass filtered), as the one shown in Fig. 1.24c). We consider parts of the signal, which fully contain exactly one wave, as denoted by the dotted lines in 
Chapter 1 - Introduction

Fig. 1.24d). For these parts of the signal we then calculate the wave thickness $d$ as the second moment of the signal based on its intensity $\gamma$, as shown below:

$$
d=\Phi^{-1} \sum_{n=1}^{N} \gamma(n) \times(n-\Theta)^{2}
$$

Note that the normalization factor $\Phi$ is the total signal intensity and $\Theta$ the first moment of the signal, defined as:

$$
\begin{aligned}
& \Phi=\sum_{k=1}^{K} \gamma(k) \\
& \Theta=\Phi^{-1} \sum_{m=1}^{M} \gamma(m) \times m
\end{aligned}
$$



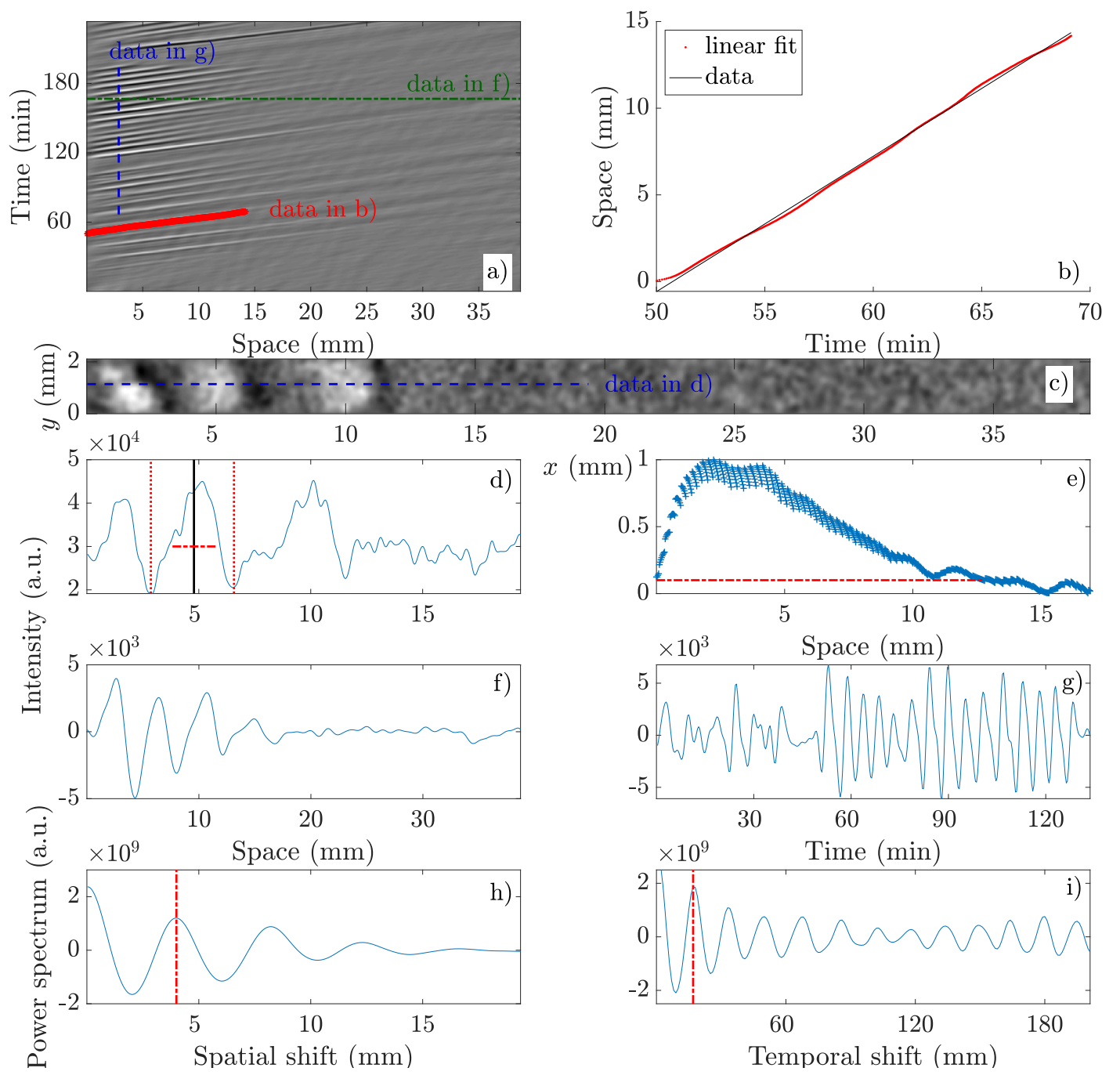

d)
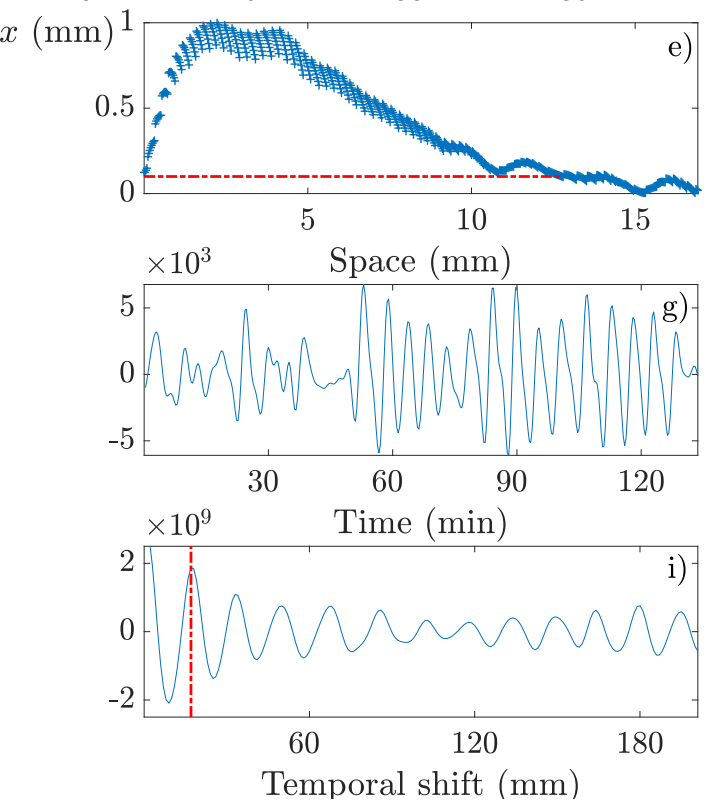

Figure 1.24: Overview of the major image analysis techniques used in this thesis. An experiment of pdsA- cells at $V_{f}=0.8 \mathrm{~mm} / \mathrm{min}$ is used as an example. a) shows the original space-time plot of the experiment. In a) the red line follows a traced wave along the channel. This data is shown as a single plot in b), together with a linear fit to the data that gives the wave speed. c) shows a snapshot of waves traveling down the channel in a normalized and band-pass filtered image. In d) a cut through the snapshot in c) is shown. The data within the two dotted lines was used to calculate the first and second moment of the wave, shown respectively as the wave center (solid line) and the wave width (dash-dotted line). In e) the intensity along the wave traced in a) is shown, normalized to its maximum. The dasheddotted line represents the wave decay length, spanning from the first point of the wave to the first point it reduces to $10 \%$ of its maximum intensity. f) and g) show slices through the data in a) for constant time or space respectively. From the auto-correlation of these signals, shown in h) and i), the wavelength and the wave period are calculated the the first maximum (shown by the dashed-dotted lines). 



\section{Influence of fast advective flows on pattern formation of Dictyostelium discoideum}

The following paper was published in PLOS ONE on the 28.03.2018 as Eckstein T, Vidal-Henriquez E, Bae A, Zykov V, Bodenschatz E and Gholami A. (2018) Influence of fast advective flows on pattern formation of Dictyostelium discoideum. PLOS ONE 13(3): e0194859. https://doi.org/10.1371/journal.pone.0194859. The paper is licensed under Creative Commons Attribution 4.0 (CC BY).

TE carried out experiments and analysed the resulting data. TE, EVH and AG designed and planned research. TE, EVH and AG wrote the initial manuscript. All authors reviewed and edited the manuscript. TE produced figures $2 \mathrm{~b}-\mathrm{d}), 3,4,5,10,11,13,15$ and 16. TE created videos S1, S2, S3, S4, S5, S10, S12, S14 and S15 from the digital supplementary material. 


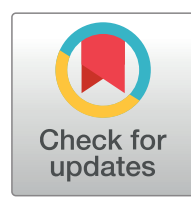

\section{G openaccess}

Citation: Eckstein T, Vidal-Henriquez E, Bae A, Zykov V, Bodenschatz E, Gholami A (2018) Influence of fast advective flows on pattern formation of Dictyostelium discoideum. PLOS ONE 13(3): e0194859. https://doi.org/10.1371/journal. pone. 0194859

Editor: Jun Ma, Lanzhou University of Technology, CHINA

Received: November 30, 2017

Accepted: March 12, 2018

Published: March 28, 2018

Copyright: ๑ 2018 Eckstein et al. This is an open access article distributed under the terms of the Creative Commons Attribution License, which permits unrestricted use, distribution, and reproduction in any medium, provided the original author and source are credited.

Data Availability Statement: All relevant data are within the paper and its Supporting Information files.

Funding: T. E. acknowledges Deutsche Forschungsgemeinschaft (DFG), project number GH 184/1-1. E.V.H. thanks the Deutsche Akademische Austauschdienst (DAAD), Research Grants-Doctoral Programs in Germany. A.G. acknowledges MaxSynBio Consortium, which is jointly funded by the Federal Ministry of Education and Research of Germany and the Max Planck

\section{RESEARCH ARTICLE \\ Influence of fast advective flows on pattern formation of Dictyostelium discoideum}

\author{
Torsten Eckstein $^{1 \odot}$, Estefania Vidal-Henriquez ${ }^{1 \odot}$, Albert Bae ${ }^{1}$, Vladimir Zykov ${ }^{1}$, \\ Eberhard Bodenschatz ${ }^{1,2,3}$, Azam Gholami ${ }^{1 *}$
}

1 Max Planck Institute for Dynamics and Self-Organization, 37077, Göttingen, Germany, 2 Institute for Nonlinear Dynamics, University of Göttingen, 37073, Göttingen, Germany, 3 Laboratory of Atomic and SolidState Physics and Sibley School of Mechanical and Aerospace Engineering, Cornell University, Ithaca, New York 14853, United States of America

- These authors contributed equally to this work.

*azam.gholami@ds.mpg.de

\begin{abstract}
We report experimental and numerical results on pattern formation of self-organizing Dictyostelium discoideum cells in a microfluidic setup under a constant buffer flow. The external flow advects the signaling molecule cyclic adenosine monophosphate (cAMP) downstream, while the chemotactic cells attached to the solid substrate are not transported with the flow. At high flow velocities, elongated CAMP waves are formed that cover the whole length of the channel and propagate both parallel and perpendicular to the flow direction. While the wave period and transverse propagation velocity are constant, parallel wave velocity and the wave width increase linearly with the imposed flow. We also observe that the acquired wave shape is highly dependent on the wave generation site and the strength of the imposed flow. We compared the wave shape and velocity with numerical simulations performed using a reaction-diffusion model and found excellent agreement. These results are expected to play an important role in understanding the process of pattern formation and aggregation of $D$. discoideum that may experience fluid flows in its natural habitat.
\end{abstract}

\section{Introduction}

In a reaction-diffusion-advection system one or more reacting species are advected downstream with an externally imposed velocity. This advective flow can induce unique emergent phenomena. An eminent example is the differential flow induced chemical instability (DIFICI) that destabilizes an otherwise spatially homogeneous state of a system [1-3]. The basic idea behind this is that the reacting species flow at different rates. This differential transport can initiate instabilities in an otherwise spatially homogeneous state of the system, leading to propagating wave packets of reactant concentrations traveling in the flow direction. This mechanism of generating spatial structures is free from the constrains of the Turing mechanism [4], which requires a large difference in diffusion coefficients of the two species involved. Accordingly one can expect DIFICI to be found widely in population dynamics [5-8] and biological morphogenesis [9]. 
Society. The funders had no role in study design, data collection and analysis, decision to publish, or preparation of the manuscript.

Competing interests: The authors have declared that no competing interests exist.
The aggregation of $D$. discoideum amoeba after nutrient deprivation is one of the best model systems for the study of spatial-temporal pattern formation at the multicellular level. Upon starvation, $D$. discoideum starts a developmental program as a surviving mechanism. The first part of this process consists of aggregation of $10^{4}-10^{5}$ chemotactic cells to form a migrating slug, which then act as a multicellular organism to search for nutrients. Because of this, $D$. discoideum has been largely studied to understand the transition from uni- towards multicellularity. The aggregation of amoebas is achieved by using the signaling chemical cyclic adenosine monophosphate (cAMP), which is initially secreted by some of the amoebas and then relayed by the others. The patterns produced by cAMP have attracted a lot of attention in the pattern formation community, since they are a primary example of spiral waves and target patterns in nature. Regarding spiral waves and target patterns in D. discoideum see for example Refs. [10-13]. These structures then constitute the centers to which the amoebas aggregate. However, in their natural habitat in the forest soil, $D$. discoideum cells are subjected to flows which advect cAMP, thus affecting the signaling process. In soils, rainwater speeds can vary from values near zero up to around $250 \mathrm{~mm} / \mathrm{min}$, which is one order of magnitude larger than flow rates studied in this work [14]. It is not yet clear how these advective flows affect the aggregation of $D$. discoideum cells in nature.

Recently, we have conducted experiments and performed numerical simulations to study flow-driven waves in a biological system, namely quasi one-dimensional colonies of signaling amoeba $D$. discoideum [15]. In these experiments with chemotactically competent $D$. discoideum cells, a straight flow-through microfluidic channel was used. Starved cells were allowed to settle on the substrate before a laminar flow of buffer was switched on. The flow advected extracellular cAMP downstream but was not strong enough to detach the cells from the substrate. This differential transport of extracellular cAMP induced macroscopic wave trains that had a unique period and propagated with a velocity proportional to the imposed flow velocity downstream. This behavior was studied theoretically $[16,17]$ using the two-component reaction-diffusion model proposed by Martiel-Goldbeter [18] for the production and relay of cAMP. While the theoretical results could explain much of the experimental observations, there were still open questions regarding the generation of a self supporting wave train at the inlet of the microfluidic channel and only small flow rates of up to $5 \mathrm{~mm} / \mathrm{min}$ were studied. Furthermore, the state of the cells was assumed to be constant in the convectively unstable regime, lacking a way to verify this experimentally.

In this work, we extend our experiments to investigate flow-driven waves at high flow rates in the same microfluidic set up. The flows are not yet strong enough to detach the cells from the substrate. We observed elongated waves that extend over the whole length of the channel and propagate both perpendicular and parallel to the flow direction. We characterized the wave shape as well as the wave propagation velocity and compared them to the numerical simulations of the system. Moreover, we changed the imposed flow rates abruptly to study the system response both experimentally and by means of numerical simulations. In our comprehensive numerical study, we found that the two-component Martiel-Goldbeter model does not correctly reproduce the wave shape observed experimentally at a higher flow velocities. However, a three-component approach successfully reproduces the wave shape while still matching with the experimental results in period and wave speed. Additionally, we found that sustained wave formation can be induced using a developmental path model [19] for the state of the cells in the channel. This seems a very reasonable assumption, since $D$. discoideum cells change the activity of a number of genes during the aggregation process [20]. We could also reproduce the experimental observations for rapid flow switching by assuming a mixture of oscillatory and excitable cells. 


\section{Materials and methods}

\section{Cell culture}

All experiments were performed with $D$. discoideum AX2-214 cells, kindly provided by Günther Gerisch (MPI for Bio-chemistry, Martinsried, Germany). Cells were grown in HL-5 medium (35.5 $\mathrm{g}$ of Formedium powder from Formedium Ltd, England, per liter of doubledistilled water, autoclaved and filtered) at $22^{\circ} \mathrm{C}$ on polystyrene Petri dishes (TC Dish 100, Sarsted, Germany) and harvested when they became confluent. Before the experiments, the cells were centrifuged and washed two times with phosphate buffer $\left(2 g\right.$ of $\mathrm{KH}_{2} \mathrm{PO}_{4}$ and $0.36 g$ of $\mathrm{Na}_{2} \mathrm{HPO}_{4} \cdot \mathrm{H}_{2} \mathrm{O}$ per liter at $\mathrm{pH}$ 6.0, autoclaved, both from Merck, Germany). The centrifuged cells were resuspended in $10 \mathrm{ml}$ of the same buffer and transferred into a shaking Erlenmeyer flask $(150 \mathrm{rpm})$ for starvation. After approximately one hour, the cells were centrifuged at $1000 \mathrm{rpm}$ for $3 \mathrm{~min}$ and resuspended in $200 \mu \mathrm{l}$ fresh phosphate buffer. The cell density was determined using a hemocytometer (Neubauer Zählkammer), diluted to $5 \times 10^{7}$ cells $/ \mathrm{ml}$ of phosphate buffer and filled into the microfluidic channel.

\section{Microfluidics}

The microfluidic devices were fabricated by standard soft lithography [21]. A silicon wafer was coated with a $100 \mu \mathrm{m}$ photoresist layer (SU-8 100, Micro Resist Technology GmbH, Berlin, Germany) and patterned by photolithography to obtain a structured master wafer. The channels are $2 \mathrm{~mm}$ wide, $50 \mathrm{~mm}$ long, and $103 \pm 2 \mu \mathrm{m}$ high. Polydimethylsiloxane (PDMS, 10:1 mixture with curing agent, Sylgard 184, Dow Corning GmbH, Wiesbaden, Germany) was poured onto the wafer and cured for $2 \mathrm{~h}$ at $75^{\circ} \mathrm{C}$. To produce the microfluidic device, a PDMS block containing the macro-channels was cut out, and two inlets $(7 \mathrm{~mm}$ and $0.75 \mathrm{~mm}$ in diameter) were punched through the PDMS at opposite ends of the channel with the help of PDMS punchers (Harris Uni-Core-7.00 and Harris Uni-Core-0.75). Afterwards, a glass microscope slide $(76 \times 26 \mathrm{~mm}, \mathrm{VWR})$ was sealed to the PDMS block following a 20-30 s treatment in air plasma (PDC 002, Harrick Plasma, Ithaca, USA) to close the macro-channels. The large inlet was used as a liquid reservoir and from the other side phosphate buffer was pumped out using a high precision syringe pump (PHD 2000 Infuse/Withdraw Syringe Pump from Harvard Apparatus, USA, combined with gasstight glass syringes from Hamilton, USA) at constant buffer flow rate. Moreover, given the dimension of the channel and the dynamics viscosity of the flowing phosphate buffer $\left(\eta=10^{-3} \mathrm{~Pa} \mathrm{~s}\right)$, one can calculate the shear stress applied on the cells at the highest imposed flow velocity of $V_{f}=50 \mathrm{~mm} / \mathrm{min}$ to be $\sigma=0.046 \mathrm{~Pa}$ (see supplementary S1 File). According to the literature, mechanosensing in D. discoideum has been observed above a threshold of $\sigma=0.7 \mathrm{~Pa}$, and cell detachment from substrate occur at higher threshold of $\sigma=2.7 \mathrm{~Pa}$ [22]. We are thus one order of magnitude below the regime where flow induced shear stress would bias the motion of chemotactic cells or even detach the cells from substrate.

\section{Image acquisition and analysis}

We used a dark-field setup consisting of a monochrome 12-bit CCD camera (QIClick-F-M-12 from QImaging), a $50 \mathrm{~mm}$ focal length objective (MVL50M23 from Thor Labs), a 7 inch focal length fresnel lens (11.0" x 11.0", 7" Focal Length from Edmund Optics, bottom side in-house coated with an anti-reflective coating) and a ring of green LEDs as light source (LED Miniatur Ringbeleuchtung LSR24-G from LUMIMAX). The camera was controlled with an image capture program (Micro-Manager [23]) and recorded images every 20 seconds. To process darkfield images, we first subtract them from each other (image number $n$ from image number 
$n+3)$ [24] and then band-passed filtered where large structures are filtered down to $3.5 \mathrm{~mm}$ and small structures up to $0.294 \mathrm{~mm}$. Finally, to calculate the phase map, at each pixel we first subtracted the time average of the signal and then performed the Hilbert transform [25].

\section{Numerical simulations}

We conducted numerical simulations of the model proposed by Martiel and Goldbeter [18] for the production and relay of CAMP, with the addition of an advection term to account for the imposed flow (see Fig 1a). The reaction-diffusion set of equations model (in 2-D) the amount of cAMP in the extracellular medium $\gamma(x, y)$, the amount of cAMP in the intracellular medium $\beta(x, y)$, and the percentage of active receptors on the outside of the cell membrane $\rho(x, y)$, where $x, y$ are cartesian spatial coordinates. This last field $\rho(x, y)$ quantifies the affinity of the cell receptors to bind with CAMP, thus providing the refractory time for this excitable medium. We use a 2-D approximation of the shape of the channel ( $x-y$ plane in Fig $2 b)$ due to its low aspect ratio in $z$ direction. The equations are as follow

$$
\begin{gathered}
\partial_{t} \rho=k_{1}\left[-f_{1}(\gamma) \rho+f_{2}(\gamma)(1-\rho)\right], \\
\partial_{t} \beta=q \sigma \alpha \Phi(\rho, \gamma) /(1+\alpha)-\left(k_{i}+k_{t}\right) \beta, \\
\partial_{t} \gamma=D \nabla^{2} \gamma-v(y) \cdot \nabla \gamma+k_{t} \beta / h-k_{e} \gamma,
\end{gathered}
$$

with

$$
f_{1}(\gamma)=\frac{1+\kappa \gamma}{1+\gamma}, \quad f_{2}(\gamma)=\frac{\mathcal{L}_{1}+\kappa \mathcal{L}_{2} c \gamma}{1+c \gamma}, \quad \Phi(\rho, \gamma)=\frac{\lambda_{1}+Y^{2}}{\lambda_{2}+Y^{2}}, \quad Y(\gamma, \rho)=\frac{\rho \gamma}{1+\gamma},
$$

$\nabla=\partial_{x} \hat{x}+\partial_{y} \hat{y}$, and $v(y)=v(y) \hat{x}$. Eq la models the process of desensitization and recovery of the active receptors given by $f_{1}$ and $f_{2}$, respectively. Eq $1 \mathrm{~b}$ characterizes the changes in cAMP
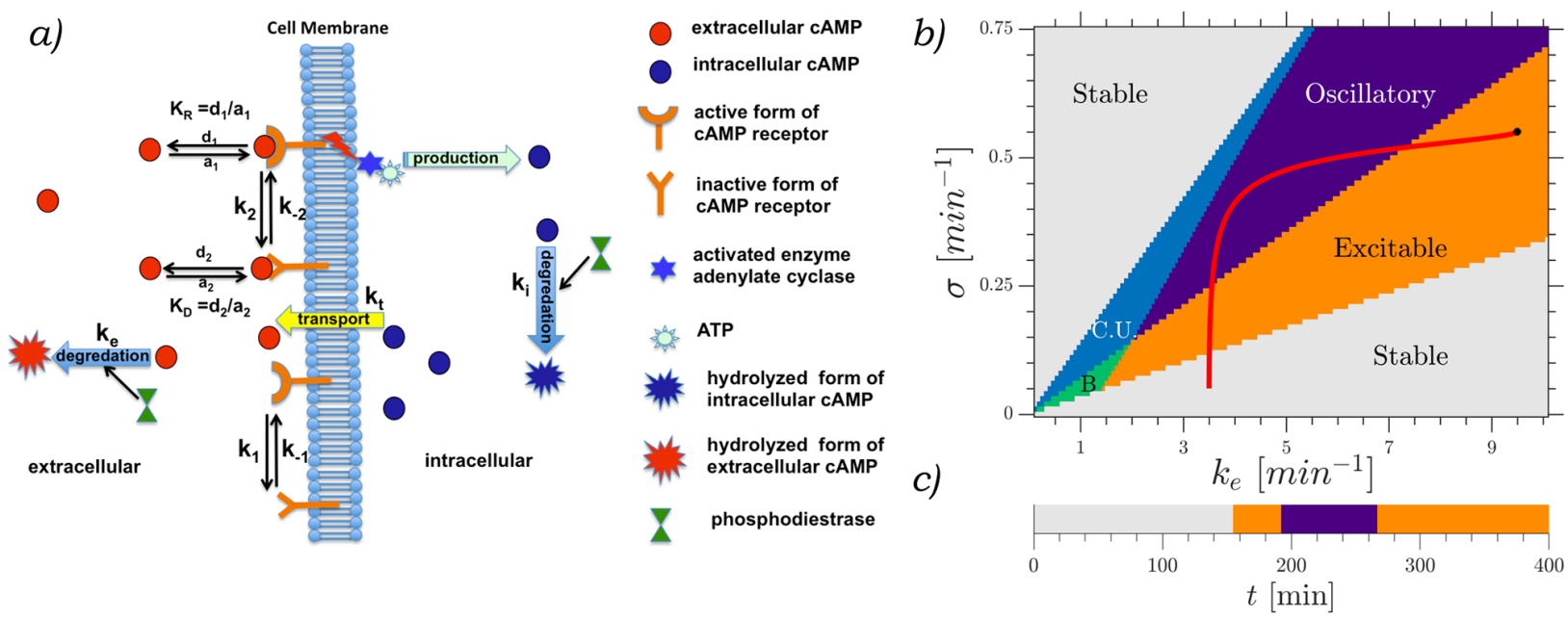

Fig 1. a) Schematic representation of the reaction-diffusion model used, reproduced from [16]. b) Phase diagram showing the different regimes depending on the production $\sigma$ and degradation $k_{e}$. Stable regime in white, where one stable steady state exists, excitable regime in orange, 3 steady states, one of which is excitable and the other two unstable. Oscillatory regime in purple, one unstable steady state surrounded by a limit cycle. Convectively unstable regime in light blue, one steady state which is convectively unstable. Bistable regime in green, two stable steady states. The red line marks the trajectory that the developmental path follows. Simulations with fixed parameters used the ones marked by the black asterisk. c) Cell state over time for a cell starting with $t_{s}=0$. The color coding is the same as b).

https://doi.org/10.1371/journal.pone.0194859.g001 


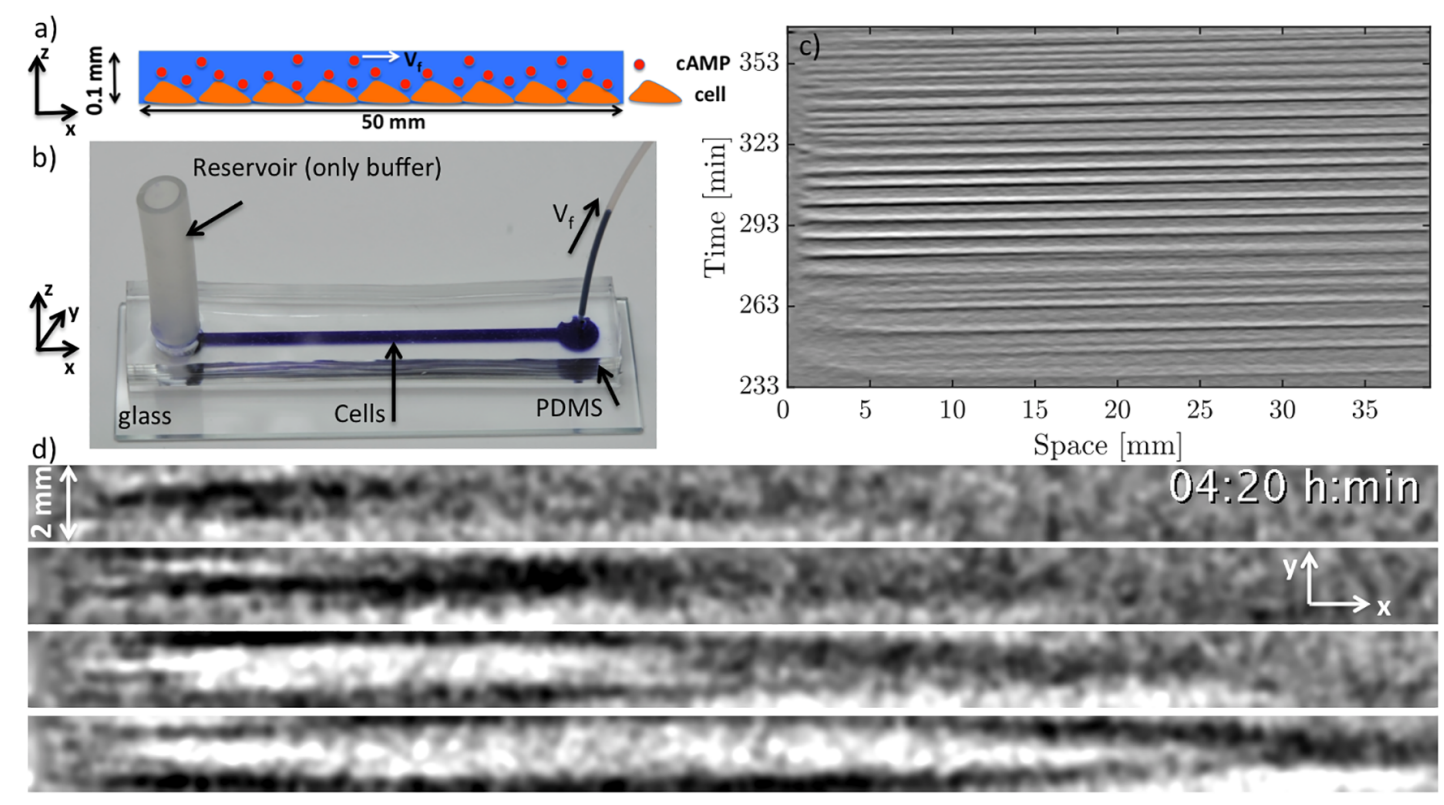

Fig 2. a) A schematic side view of the channel loaded with cells that are attached to the substrate and exposed to an external fluid flow advecting cAMP molecules downstream. b) Experimental setup filled with blue ink for better visualization. The reservoir is filled only with buffer and the liquid is pumped out with a syringe pump from the right side. c) Space-time plot of the flow-driven waves at the imposed flow velocity of $V_{f}=10 \mathrm{~mm} / \mathrm{min}$. d) Snapshots of the waves taken from the top of the channel obtained by subtracting successive images (captured every $20 \mathrm{sec}$ ) of the channel every 1 min (image number $n+3$ minus image number $n$ ) and bandpass filtered. The time increment between successive images is $1 \mathrm{~min}$. Time stamp shows the time since the start of starvation.

https://doi.org/10.1371/journal.pone.0194859.g002

inside the cells given by the nonlinear production term $\Phi$. The amount of intracellular cAMP is reduced by intracellular degradation and transport to the extracellular medium at rates described by $k_{i}$ and $k_{t}$, respectively. Finally, Eq $1 \mathrm{c}$ represents the changes on $\gamma$ given by degradation through phosphodiesterase at a rate $k_{e}$ and the transport from the intracellular medium at a rate $k_{t}$. These processes are schematically represented in Fig 1a. $\gamma$ is subjected to diffusion and advection, the other two fields do not diffuse nor advect since they are attached to the cells. For a detailed derivation of this model please refer to the original works of Martiel and Goldbeter [18] and Tyson et. al. [26]. The parameters used are $k_{1}=0.09 \mathrm{~min}^{-1}, \kappa=18.5$, $\mathcal{L}_{1}=10, \mathcal{L}_{2}=0.005, c=10, q=4000, \alpha=3, \lambda_{1}=10^{-4}, \lambda_{2}=0.2575, k_{i}=1.7 \mathrm{~min}^{-1}, k_{t}=0.9$ $\min ^{-1}, D=0.024 \mathrm{~mm}^{2} / \mathrm{min}, h=5$.

We simulated this system using a Runge-Kutta scheme with a Merson error aproximation [27] to ensure numerical accuracy. Nonlinear discretization was used for the advection operator in order to deal with high velocities while keeping a non-negative concentration of cAMP [28]. We used a no flux $\left(\partial_{x} \gamma(x=0)=0\right)$ boundary condition in all boundaries, including upstream, and kept the same parameters as in our previous simulations [29] while keeping freedom to move in the parameter space characterized by $\sigma$ and $k_{e}$. We conducted simulations both with fixed parameters (over time and space) and with a developmental path based on the work by Laurenzal et al [19]. When we used this path, the parameters $k_{e}$ and $\sigma$ were changed from being uniform in the whole system to being particular to each cell group (patch). Each patch had an area of $0.1 \mathrm{~mm} \times 0.1 \mathrm{~mm}$ and a particular starting time along the cellular developmental path. This path takes the cells from having one stable solution, to an excitable regime, one oscillatory solution, and then back to excitable (see Fig $1 \mathrm{~b}$ for an overview of the 
different regimes in this system), by changing with time the parameters $\sigma$ and $k_{e}$ according to

$$
\sigma(t)=0.3+0.25 \tanh \left(\frac{t+t_{s}-200}{50}\right), \quad k_{e}(t)=6.5+3 \tanh \left(\frac{t+t_{s}-260}{30}\right),
$$

where $t_{s}$ corresponds to the initial development time of a patch and $t$ is the simulation time. The starting times were selected following an exponential distribution with a rate parameter $\Delta^{-1}$

$$
P\left(t_{s}\right)=\frac{e^{-t_{s} / \Delta}}{\Delta}
$$

In all our simulations $\Delta=25 \mathrm{~min}$. The advection velocity $V_{f}$ was selected to be constant along the longest axis of the channel ( $x$-axis) while the $y$-axis dependency was calculated using the Navier-Stokes equation with the assumption of a laminar Pouseuille flow. This gives a flow that is mostly planar with a sharp drop at the boundaries, with a boundary layer of about 50 $\mu \mathrm{m}$, which is of the order of half the height of the channel (see supplementary information). The system was initiated with each patch at its steady state. Different initial states were tested and did not seem to influence the final results, since the system quickly relaxes to its steady state.

We also performed simulations using the two-component version of this model, which makes the assumption that the intracellular production of cAMP is immediately transported to the extracellular medium. This is achieved numerically by setting $\partial_{t} \beta=0$, thus the set of equations becomes

$$
\begin{gathered}
\partial_{t} \gamma=D \nabla^{2} \gamma-v(y) \cdot \nabla \gamma+s \Phi(\rho, \gamma)-k_{e} \gamma, \\
\partial_{t} \rho=k_{1}\left[-f_{1}(\gamma) \rho+f_{2}(\gamma)(1-\rho)\right],
\end{gathered}
$$

where $s=q k_{t} \alpha \sigma /\left(h\left(k_{t}+k_{i}\right)(1+\alpha)\right)$. All parameters used are the same as in Eq 1 .

\section{Results \\ Characterization of the flow-driven waves at high flow rates}

In the absence of flow, signaling $D$. discoideum cells synchronize and show formation and propagation of spiral waves (see supplemental S1 Video). When subjected to advective flows, the spiral patterns are replaced by wave trains traveling downstream. Fig $2 \mathrm{~d}$ shows an example of flow-driven waves for an average flow velocity of $V_{f}=10 \mathrm{~mm} / \mathrm{min}$. The image contrast reflects the shape changes of the cells. The light bands correspond to high concentrations of cAMP and consist of elongated cells while in the dark bands the cAMP concentrations is small and cells remain round [12, 30-32].The corresponding space-time plot is shown in Fig 2c, where light intensity is averaged over the $2 \mathrm{~mm}$ width of the channel and then stacked up along the time axis. The slope of the diagonal bands give the inverse of the average propagation velocity of the waves along the channel. The wave shape and propagation speed strongly depend on the strength of the imposed flow velocity. At small flow rates, a wave train develops spontaneously that fills the whole length of the channel (Fig 3a and supplementary S2 Video). The wavelength of the traveling waves increases linearly with the imposed flow velocity [15] and becomes comparable or larger than the length of the microfluidic channel at high flow rates (Fig $3 b-3 d$ ). Deformations of the wave front also increase significantly with the imposed flow velocity. Planar wave fronts at small flow rates deform to parabolic fronts at intermediate velocities and become extremely extended at higher flow speeds (Fig 3b-3d, and supplementary S3, S4 and 


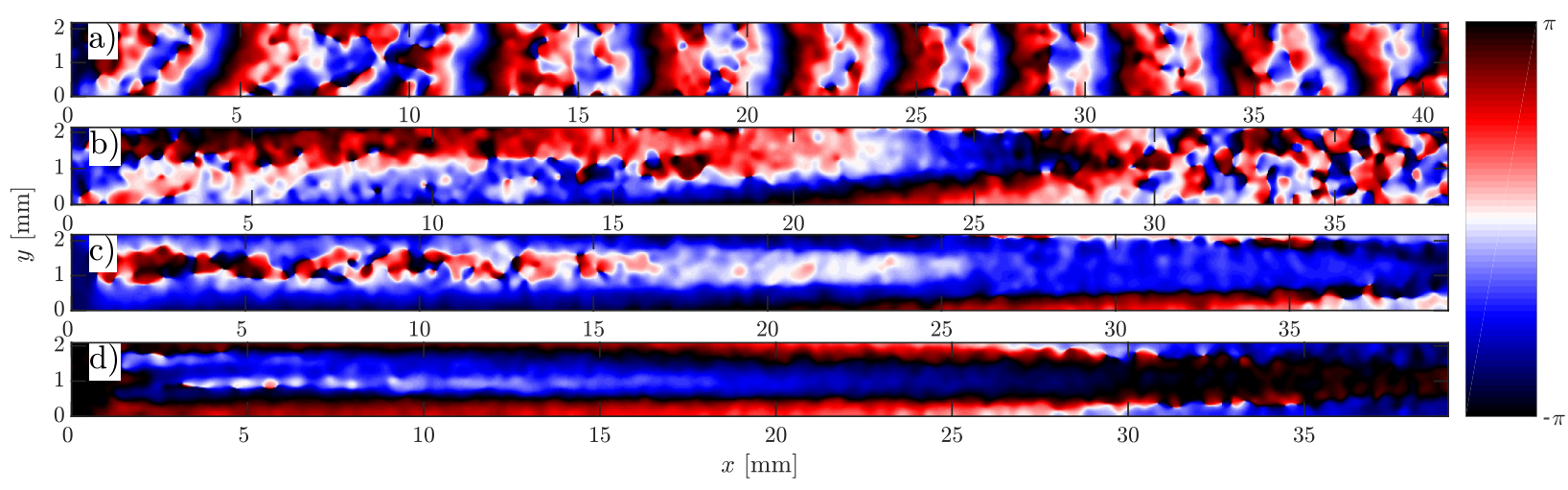

Fig 3. Phase map of the flow-driven waves showing different wave shapes at different imposed flow velocities. a) Practically planar wave fronts at $V_{f}=0.5 \mathrm{~mm} / \mathrm{min}$. b) Parabolic shape at $V_{f}=5 \mathrm{~mm} / \mathrm{min}$. Extremely elongated parabolic wave fronts at flow velocities of $V_{f}=10 \mathrm{~mm} / \mathrm{min}$ and $V_{f}=15$ $\mathrm{mm} / \mathrm{min}$ are shown in c) and d), respectively.

\section{https://doi.org/10.1371/journal.pone.0194859.g003}

S5 Videos). Notice that the wave shape does not reflect the flow profile which is relatively constant across the width of the channel and drops quickly to zero at a length scale comparable to half of the channel's height $(50 \mu \mathrm{m})$, see supplementary S1 Fig and Ref. [29] for detailed calculations of the flow profile. Moreover, at small and intermediate flow rates, the waves propagate solely in the flow direction. However, at higher flow rates they propagate both in the flow direction as well as transversal to the imposed flow. While propagation speed along the flow $\left(v_{\|}\right)$is comparable to the imposed flow velocity, the transversal propagation speed $\left(v_{\perp}\right)$ is much smaller and of the order of the propagation velocity of waves emitted by spirals in this system in the absence of flow $\left(v_{\perp, \text { avg }}=0.45 \pm 0.04 \mathrm{~mm} / \mathrm{min}\right.$ ). The wave period $T$ shows no clear velocity dependence, and takes on a value of $T_{\text {avg }}=5.98 \pm 0.25 \mathrm{~min}$. We also measured the width of the wave fronts $d$, as a function of the imposed flow velocity. We found a linear dependency which is shown in Fig 4d. For this measurement, we calculated the second moment of the light intensity $I$ at the middle of the channel defined as $\sigma^{2}=\sum_{i} I_{i}\left(x_{i}-\bar{x}\right)^{2} / \sum I_{i}$, and multiplied $\sigma$ by the factor of 2.355 to obtain $d$ as the "full width at half maximum" (FWHM) of a Gaussian distribution with standard deviation $\sigma$.

At high flow velocities, the wave generation site plays an important role for its final shape. If the wave is initiated close to the vertical middle of the channel, it propagates along the length and across the width of the channel. Since the wave propagation velocity parallel to the flow is
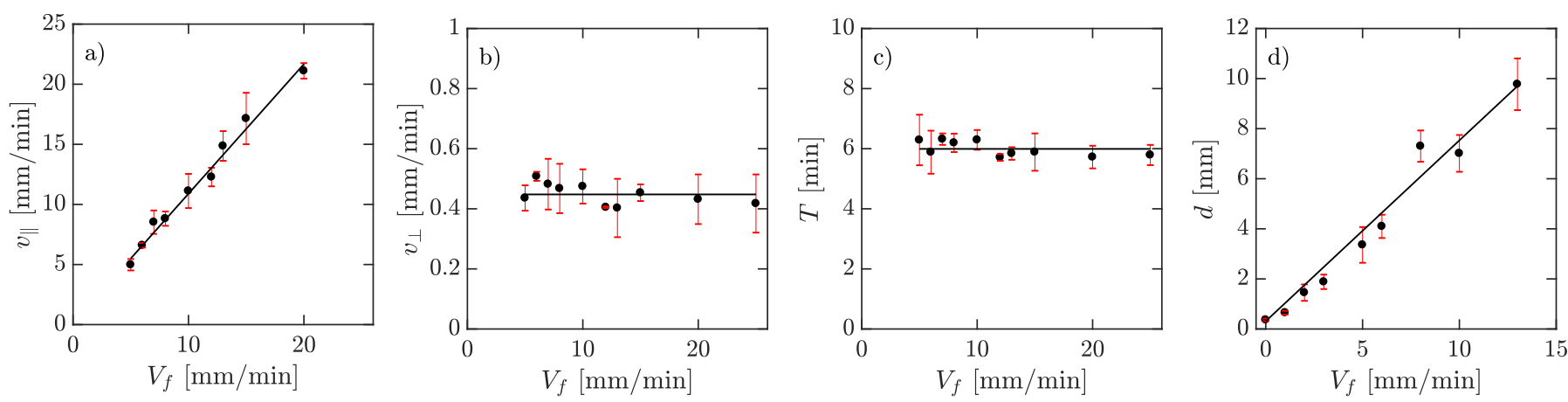

Fig 4. Experimental data on dependency of a) wave speed along the channel $v_{\|}$, b) wave speed in transversal direction $v_{\perp}$, c) wave period $T$, and d) wave front thickness as a function of imposed flow velocity $V_{f}$. Continuous lines represent in a), d) least square fit assuming linear scaling and in b), c) average transversal propagation velocity and wave period.

https://doi.org/10.1371/journal.pone.0194859.g004 


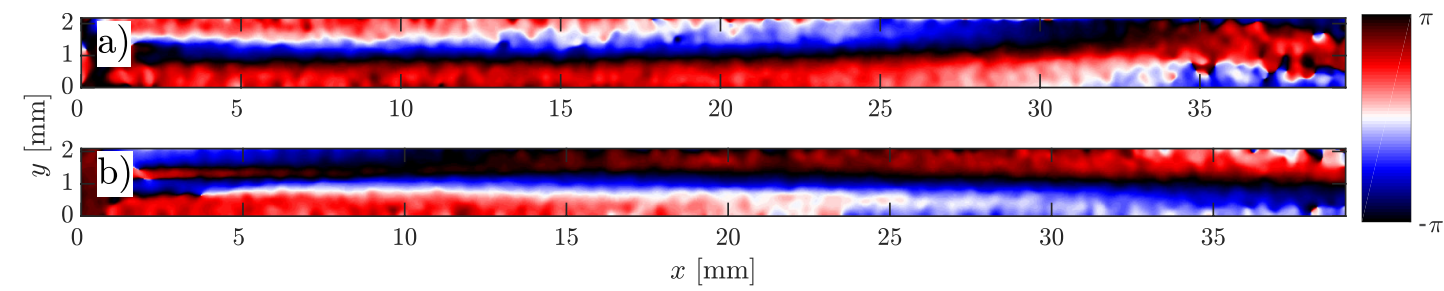

Fig 5. a) A half-parabolic shaped wave front observed at $V_{f}=10 \mathrm{~mm} / \mathrm{min}$. b) Two stripe-like waves initiating at top and bottom boundaries for flow speed of $V_{f}=15 \mathrm{~mm} / \mathrm{min}$.

https://doi.org/10.1371/journal.pone.0194859.g005

much faster than perpendicular to it, the wave gets stretched along the channel. This leads to the formation of an elongated parabolic-shaped wave front (Fig 3c and supplemental S4 Video). However, if the initial excitation is in the vicinity of top $(y=2 \mathrm{~mm})$ or bottom boundaries $(y=0)$, the wave can only propagate in one direction across the channel, which results in a half-parabola wave front, as shown in Fig 5 a and supplemental S4 Video. At very high speeds $\left(V_{f} \geq 15 \mathrm{~mm} / \mathrm{min}\right.$ ), we observe an extreme version of this process where stripe-like patterns form, as shown exemplary in Fig $5 \mathrm{~b}$ for $V_{f}=15 \mathrm{~mm} / \mathrm{min}$ and supplemental S5 Video.

\section{Numerical simulations results}

To study the wave shape in our system in a more detailed manner, we performed numerical simulations of the two-component model (Eq 2) at high flow speeds and fixed parameters $\sigma=$ $0.55 \mathrm{~min}^{-1}, k_{e}=9.5 \mathrm{~min}^{-1}$ (excitable regime). Starting with an initial perturbation centered upstream in the channel, we observed that the produced waves do not acquired a parabolic shape, but rather a planar form very similar to the flow profile applied as it is shown in Fig 6a and supplementary S6 Video. We compared these patterns to simulations of inert particles being advected with the same flow, and found very good agreement between the two as shown in the top two panels of Fig 6 . This direct correspondence between the wavefront evolution and the advection velocity is due to the instantaneous reaction of the cells to the extracellular presence of cAMP.

In contrast to the two-component model, simulations of the three-component model (Eq 1) with the same parameters gave a shape much more similar to the experiments as can be

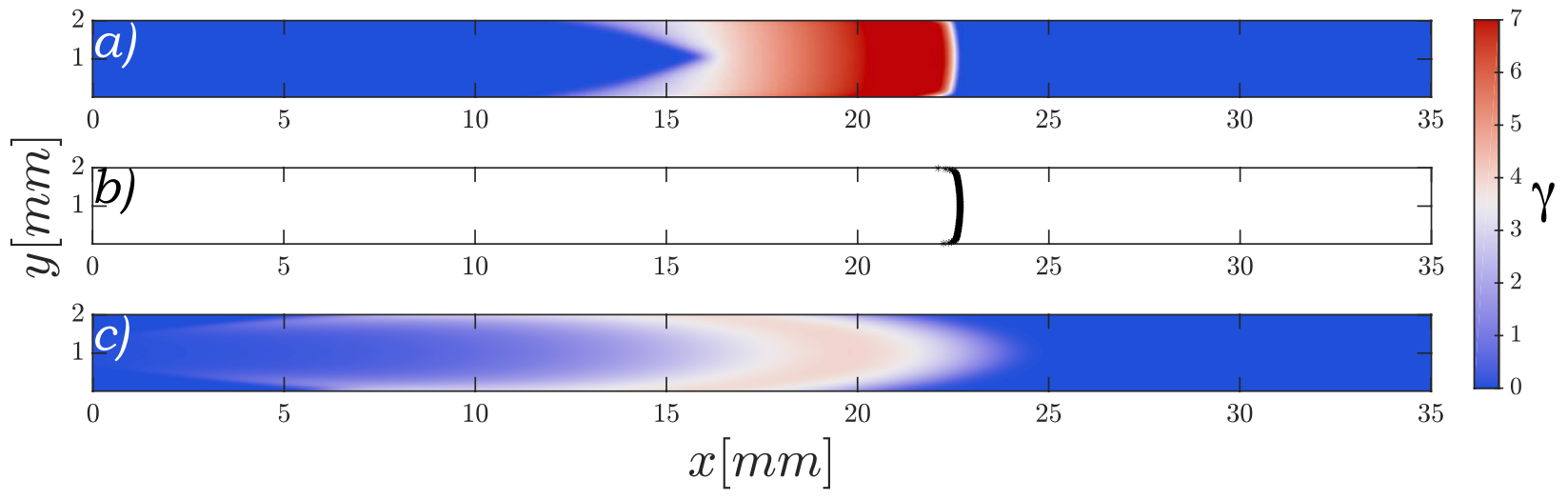

Fig 6. Comparison of wave shapes. For a) the two-component model and c) the three-component model an initial perturbation was applied center upstream on the channel and advected at $V_{f}=10 \mathrm{~mm} / \mathrm{min}$. Panel b) shows a group of particles with no interaction between them starting at the same position as the perturbation in a) and being advected at $V_{f}=v_{\perp}+10 \mathrm{~mm} / \mathrm{min}$, with $v_{\perp}=1.8 \mathrm{~mm} / \mathrm{min}$ the velocity of the two-component model wave without advection.

https://doi.org/10.1371/journal.pone.0194859.g006 

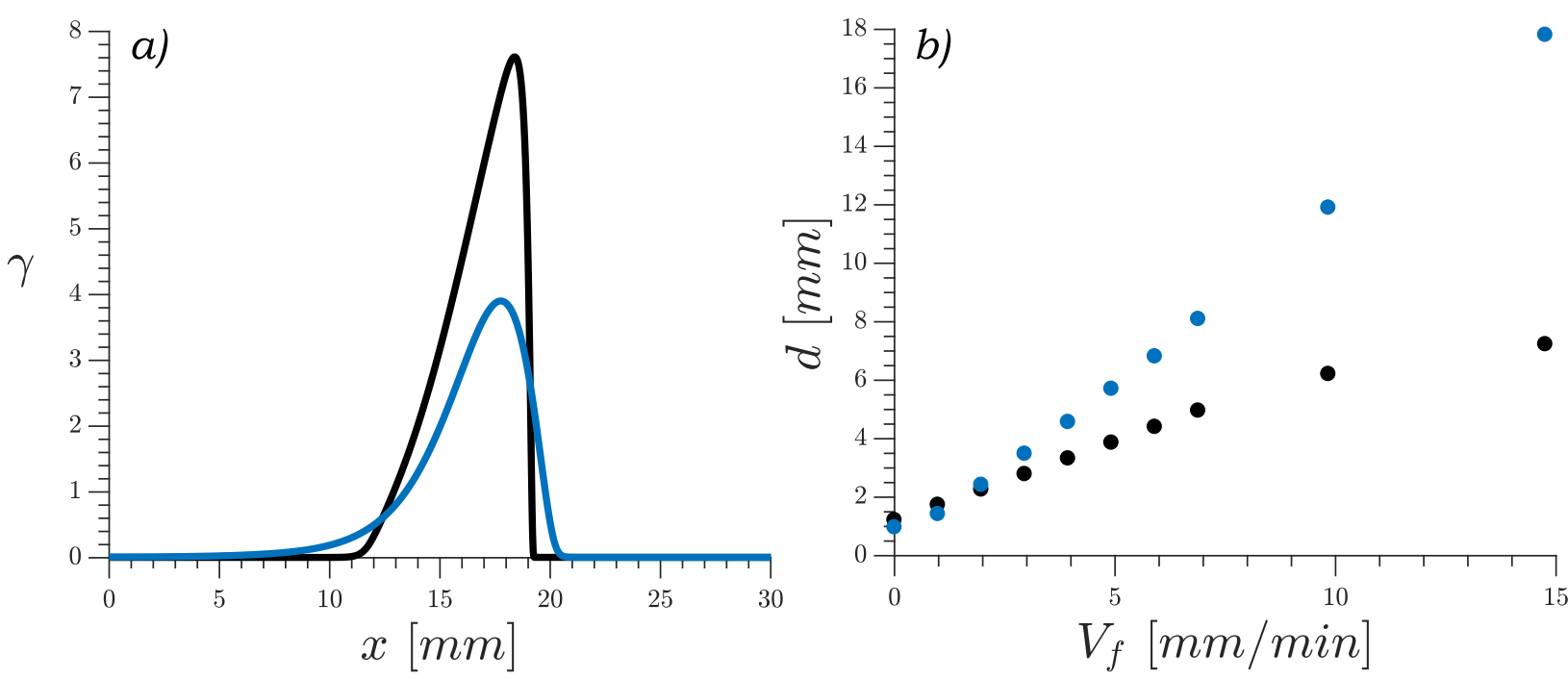

Fig 7. a) Wave profile comparison between the two- and three-component models at imposed flow velocity of $V_{f}=5 \mathrm{~mm} / \mathrm{min}$. b) Wave thickness vs imposed flow for the different models; calculated as 2.355 times the square root of the second moment. Two-component model in black, threecomponent model in blue.

https://doi.org/10.1371/journal.pone.0194859.g007

seen in Fig 6c and supplementary S7 Video. In this version of the model there is a non-instantaneous transport of cAMP between the intracellular and the extracellular media, thus slowing down the waves and effectively shrinking the difference between the velocities across and along the channel, allowing for more rounded shapes. The striking difference between the waves generated by the two models can also be appreciated in the wave profile under advective flow shown in Fig 7a. Here it can be seen that in the fast dynamics model the front of the wave is very sharp, with the cAMP rising to its maximum value very quickly. In the three-component model the wave build up is much slower showing a softer curve that looks more similar to our experimental observations.

We found the velocity of the observed waves to increase linearly with the applied flow for both models, in agreement with the experiments. We also observed an increase of the thickness on the wave profile with increased advection flow. To characterize this, we calculated $2.355 \sigma$, where $\sigma^{2}$ is the second moment of the wave along the middle of the channel defined as $\sigma^{2}=\sum \gamma_{i}\left(x_{i}-\bar{x}\right)^{2} / \sum \gamma_{i}$. These results are shown in Fig $7 \mathrm{~b}$. The increase is faster in the three-component model than in the two-component one, consistent with the profile shown in Fig $7 a$.

Finally, we performed simulations with cells following a developmental path as described in Materials and Methods. Similarly as previously observed in [33], we see cAMP waves starting from cells more advanced in their developmental path, i.e. higher $t_{s}$. When we tried varying the patch size we observed that a minimum amount of cells together in the oscillatory regime were necessary to initiate a wave. For bigger patches, one patch was enough to initiate a wave. Interestingly, we observed that at high speeds (above $2 \mathrm{~mm} / \mathrm{min}$ ) only oscillatory patches at the left end (upstream) of the channel generate waves. Advanced cell clusters down the channel failed to produce waves. The wave shapes observed were of a wide variety, very similar to the ones observed in experiments. The numerical waves presented in Figs 8 and 9 can be compared to the experimental ones of Fig 3, showing very elongated parabolic-shape waves and waves moving perpendicularly to the flow (see supplementary S8 and S9 Videos). 


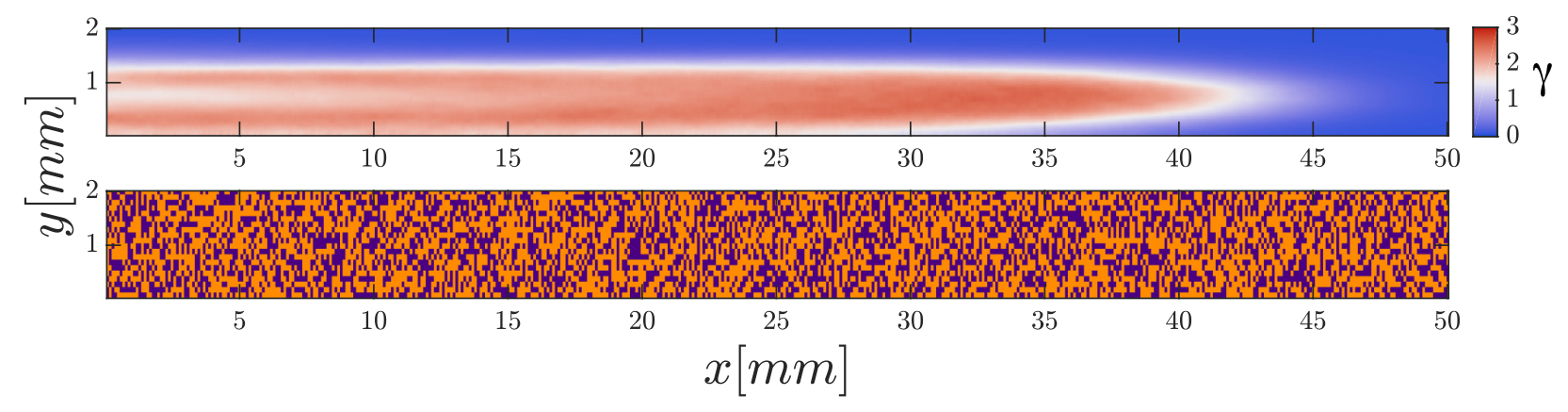

Fig 8. Parabolic-shaped wave observed in simulations using a developmental path. Advecting flow $V_{f}=15 \mathrm{~mm} / \mathrm{min}$. Top: $\mathrm{cAMP}$ concentration. Bottom: State of the cells at the moment of wave initiation: Excitable cells in orange and oscillatory cells in purple. The wave is initiated upstream almost at the middle of the channel.

https://doi.org/10.1371/journal.pone.0194859.g008

\section{On- and off- cycles of the imposed flow}

To verify our assumptions on the dynamical state of the cells in the numerical simulations, we performed experiments in which we abruptly switched off the imposed flow, after the flowdriven waves had been fully established throughout the channel. This lets us to distinguish between real waves of cAMP and phase waves, as both types of waves respond differently to changes in flow rate. If the cells are mostly in the oscillatory regime, we expect the waves to be phase waves. Since a phase wave is not directly induced by the diffusing chemicals, it should travel at the same velocity and width after turning off the flow. In contrast, an excitation (trigger) wave should propagate at the normal speed selected nonlinearly by the reaction-diffusion balance of the system and should also recover its standard width in the absence of the flow. Finally, it is also possible that the waves are not stable under abrupt changes of the flow rate.

Thus, we performed experiments in which we switched off the imposed flow while there were flow-driven waves clearly visible in the channel. The corresponding space-time plot of this experiment is shown in Fig 10. We find that in the presence of an external flow, the waves have a higher amplitude as it can be seen in Fig 10 and supplementary S10 Video. The thickness of the wave fronts becomes two to three times larger in the presence of flow (see Fig 11). For a number of experiments, we observed that the waves in the channel would slow down and travel further along the channel with their typical velocity of $0.4 \mathrm{~mm} / \mathrm{min}$ in the absence of advection, as shown in Fig 11 and S10 Video. However, these waves usually did not traverse the channel very far, being annihilated by emitted waves from newly formed centers. These

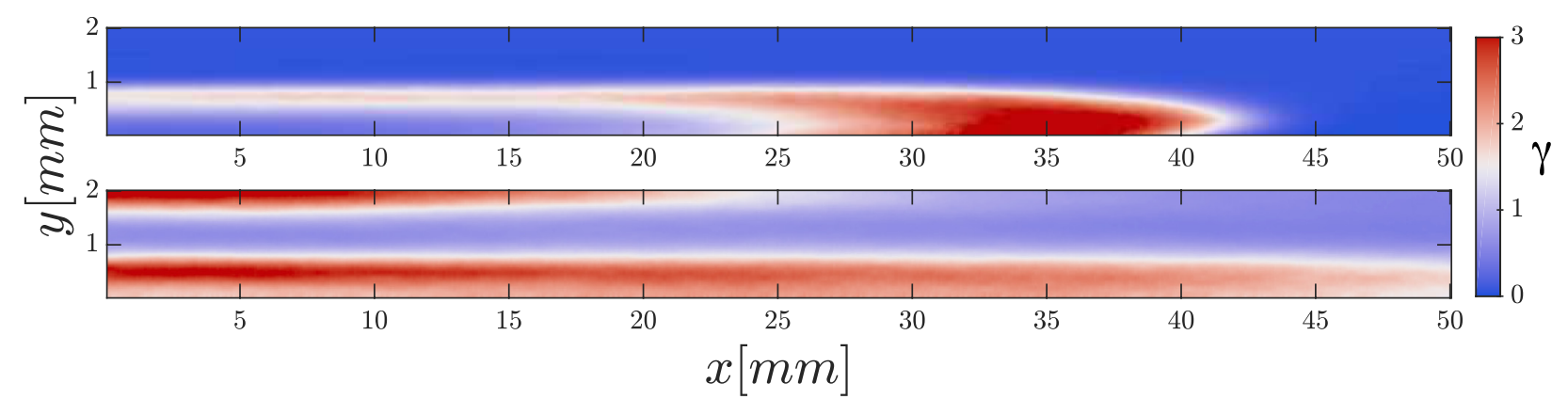

Fig 9. Elongated waves observed in simulations using a developmental path. Advecting flow $V_{f}=15 \mathrm{~mm} / \mathrm{min}$. Top and bottom panels show cAMP waves in simulations with two different initial conditions in the state of the cells.

https://doi.org/10.1371/journal.pone.0194859.g009 


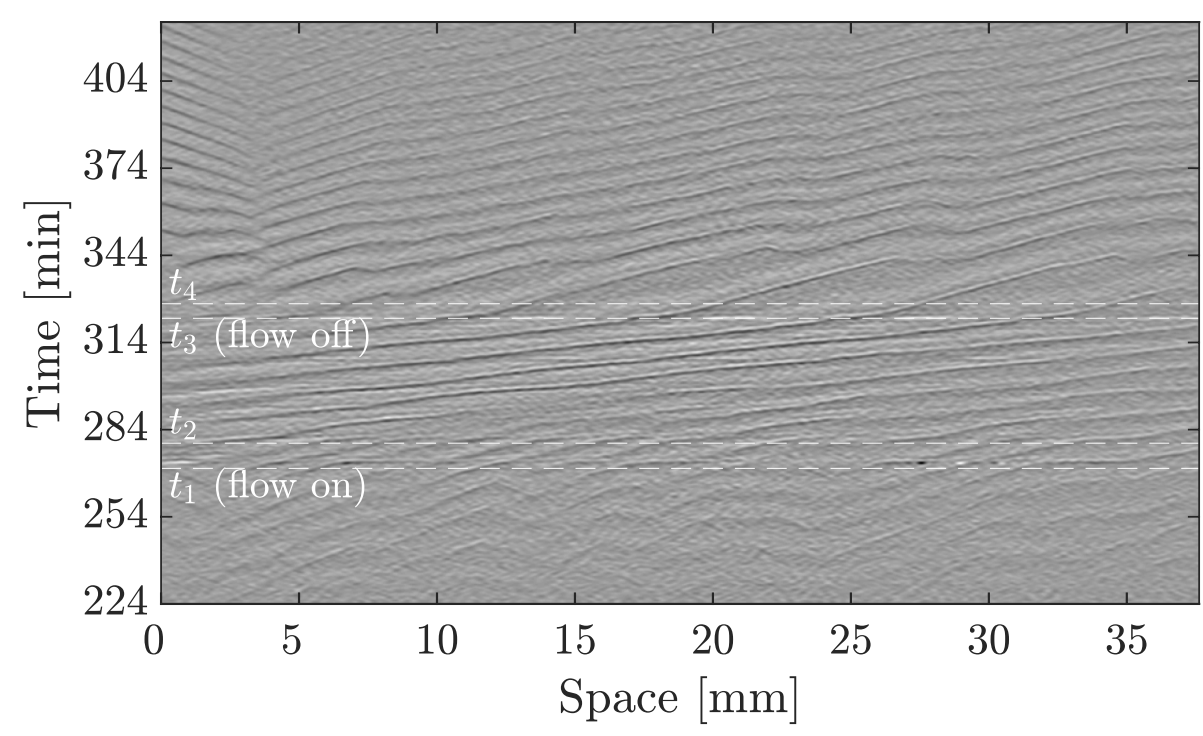

Fig 10. Space-time plot of an experiment in which the flow was initially absent, then turned on $\left(V_{f}=1 \mathrm{~mm} / \mathrm{min}\right)$ at $t_{1}$ and turned off again at $t_{3}$. While the flow is off $\left(t \leq t_{1}\right)$, the cells show target patterns. After it turns on at $t_{1}$, there is a short disordered phase until flow-driven waves fully develop, which travel downstream at $v_{\|, \text {on }}=0.99 \pm 0.03$ $\mathrm{mm} / \mathrm{min}$. At time $t_{3}$, the flow is turned off and the waves still propagate further downstream at slower speed of $v_{\|, \text {off }}=0.37 \pm 0.03 \mathrm{~mm} / \mathrm{min}$ for $30 \mathrm{~min}$. They ultimately vanish on collision with waves emitted from new centers.

https://doi.org/10.1371/journal.pone.0194859.g010

observations confirm that these propagating waves are trigger waves and at least a portion of the cells are in the excitable regime.

We also performed numerical simulations with a similar setup, that is, with a developmental path scheme and switching off the flow once the waves were formed. Results from those simulations are presented as a space-time plot in Fig 12 and supplementary S11 Video. We observed a change in wave thickness and velocity once the flow is switch off, with some waves continuing traveling at a smaller speed, showing good agreement with the experimental observations.

Finally, we increased step-wise the imposed flow velocity to further study the system response. Fig 13 shows the space-time plot of an experiment where the imposed flow increases from $1 \mathrm{~mm} / \mathrm{min}$ to $4 \mathrm{~mm} / \mathrm{min}$ and returns back to $1 \mathrm{~mm} / \mathrm{min}$ at the end. The slope of the
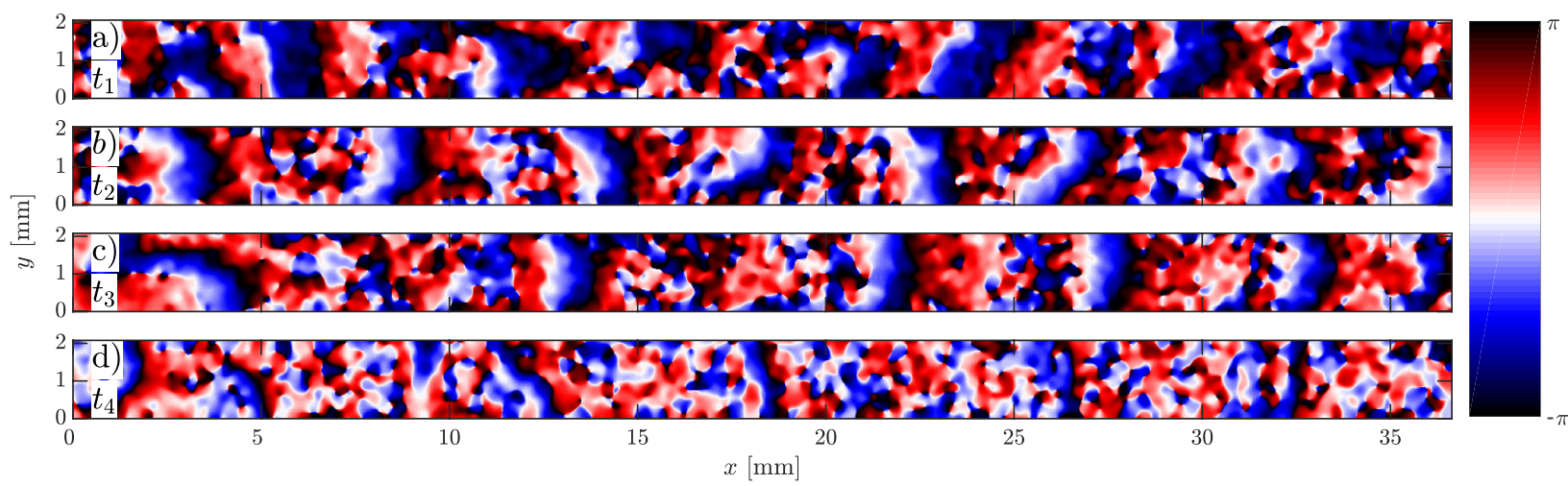

Fig 11. a) The wave pattern at the time point that the flow of magnitude $V_{f}=1 \mathrm{~mm} / \mathrm{min}$ is turned on $\left(t_{1}\right.$ in Fig 10), and b) at time $t_{2}, 8 \mathrm{~min}$ later. c) The fully developed waves shortly before turning off the flow at time $t_{3}$ and $\mathrm{d}$ ) the waves at time $t_{4}$, shortly after switching off the flow.

https://doi.org/10.1371/journal.pone.0194859.g011 


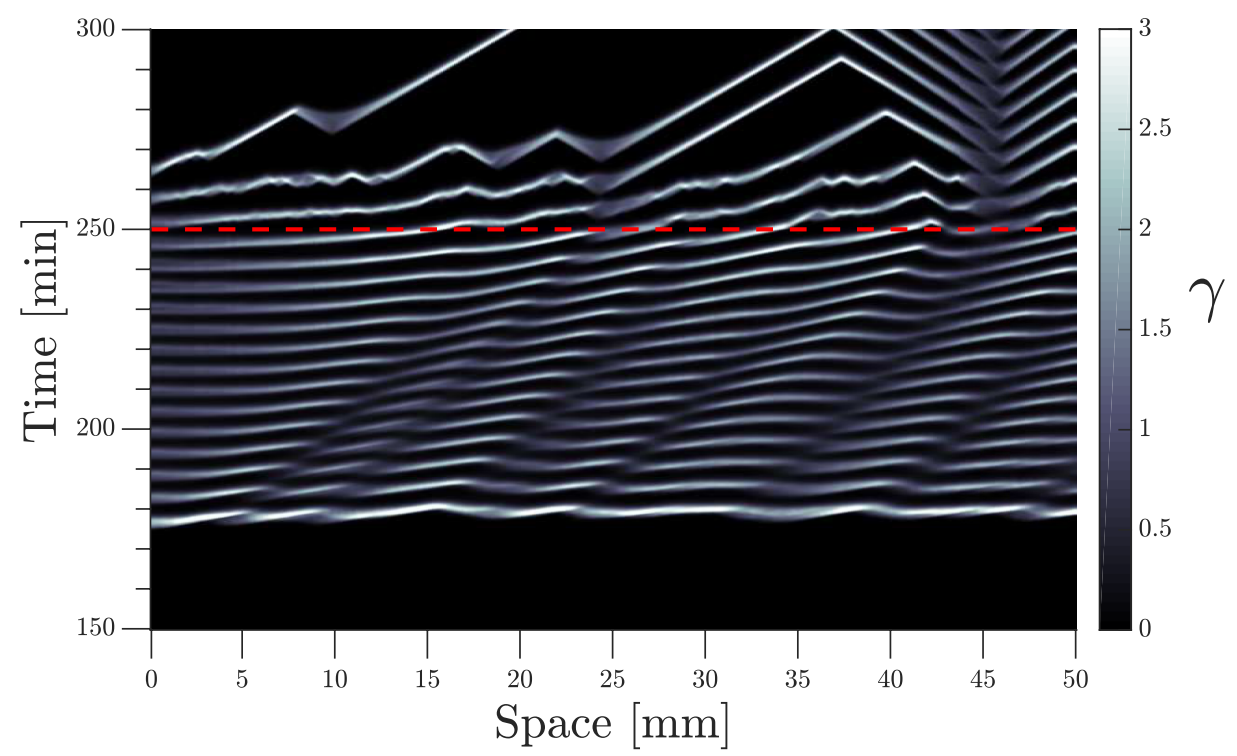

Fig 12. Space-time plot of numerical simulations using the developmental path scheme. The advecting flow is initially $V_{f}=1 \mathrm{~mm} / \mathrm{min}$ and stops at $t=250 \mathrm{~min}$.

https://doi.org/10.1371/journal.pone.0194859.g012

diagonal bands, which give a measure of the inverse wave velocity, follow the velocity jumps of the applied flow (see supplementary S12 Video). Interestingly, we observe a transient decrease in the wave period as the imposed velocity changes from $2 \mathrm{~mm} / \mathrm{min}$ to $3 \mathrm{~mm} / \mathrm{min}$. Since the wavelength is already fixed for the previously developed waves at $2 \mathrm{~mm} / \mathrm{min}$, they adjust to higher speed by decreasing the period to $4 \mathrm{~min}$ (roughly $2 / 3$ of the normal $6 \mathrm{~min}$ period).

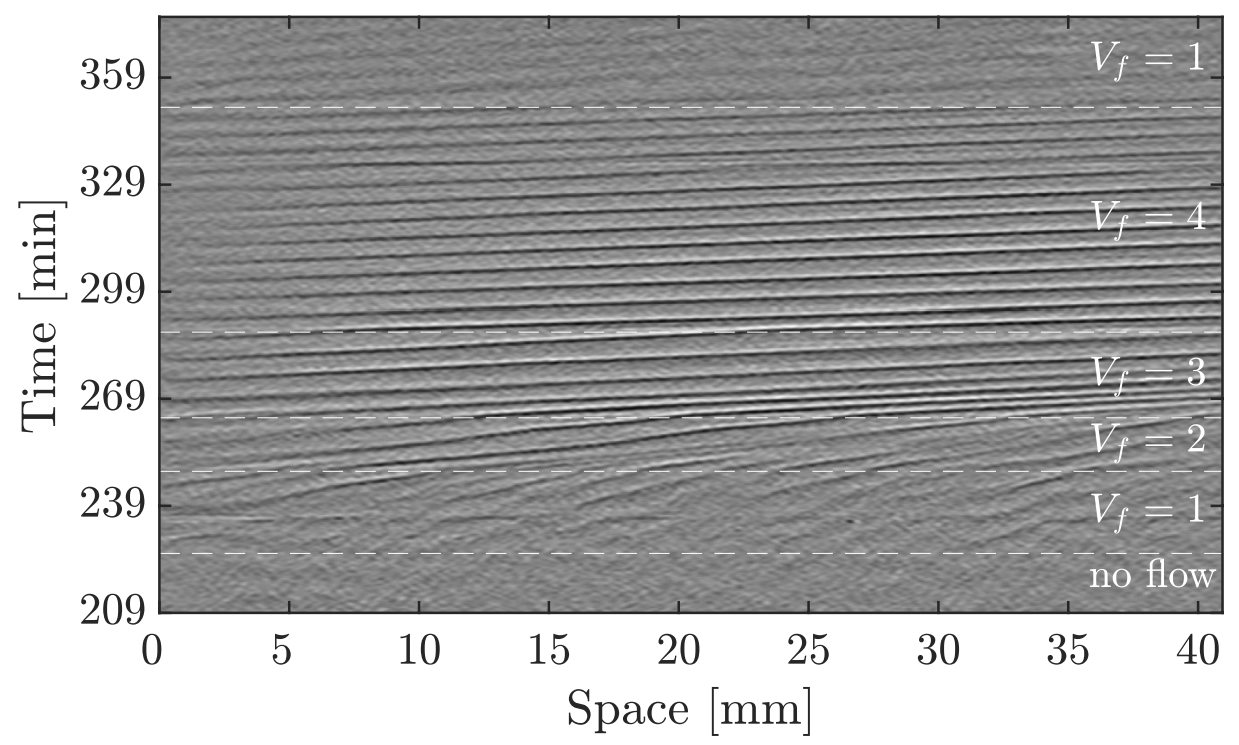

Fig 13. Propagating waves follow the velocity jumps of the imposed flow from $1 \mathrm{~mm} / \mathrm{min}$ to $4 \mathrm{~mm} / \mathrm{min}$. The waves already established at $V_{f}=2 \mathrm{~mm} / \mathrm{min}$ accelerate as the flow increases to $3 \mathrm{~mm} / \mathrm{min}$, and to keep the wavelength already set at $V_{f}=2 \mathrm{~mm} / \mathrm{min}$, period decreases transiently for these waves to $4 \mathrm{~min}$.

https://doi.org/10.1371/journal.pone.0194859.g013 


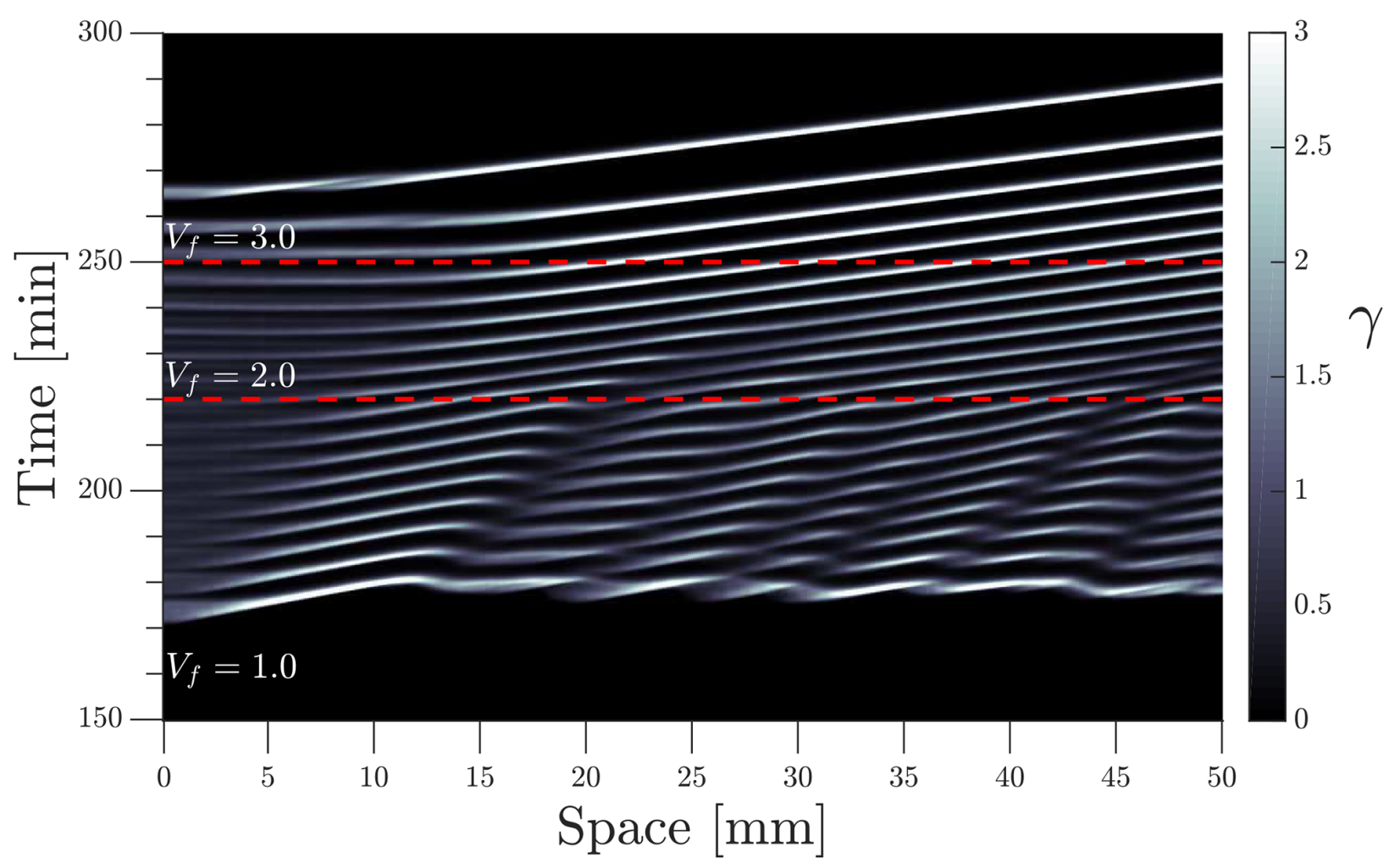

Fig 14. Space-time plot of numerical simulations using the developmental path scheme and a stepwise incremental flow. Initial flow velocity $V_{f}=$ $1.0 \mathrm{~mm} / \mathrm{min}$, incremented at $t=220 \mathrm{~min}$ to $V_{f}=2.0 \mathrm{~mm} / \mathrm{min}$, and again increased to $V_{f}=3.0 \mathrm{~mm} / \mathrm{min}$ at $t=250 \mathrm{~min}$.

https://doi.org/10.1371/journal.pone.0194859.g014

Newly developed waves at the inlet area of the channel ( $\left.V_{f}=3 \mathrm{~mm} / \mathrm{min}\right)$, have a higher wavelength and velocity, and the wave period recovers back to the standard value of $6 \mathrm{~min}$.

Numerical simulations of a similar system with developmental path scheme is shown in Fig 14 and supplementary S13 Video, where the flow velocity is increased stepwise from $1 \mathrm{~mm} /$ $\min$ to $3 \mathrm{~mm} / \mathrm{min}$.

\section{Aggregation under the influence of flow}

To investigate aggregation dynamics of $D$. discoideum cells in the presence of flow, we performed experiments in which the flow is maintained well into the culmination phase of the life cycle. In our experiments, waves appear 3-6 hours after starvation. During this time, chemotactic cell movement is still weak [34] and the variations in cell density are not significant (compare Fig 15a and 15b). Later, 8 hours into starvation, cells form atypical aggregate patterns at high flow rates, as shown in Figs $15 \mathrm{c}$ and 16e. Similar to the experiments in Ref. [35], we observed cone-shaped long streams that existed in the downstream and lateral side of the centers. The lateral streams continue to line up in the direction of the imposed flow (see Fig $15 \mathrm{~d}$ and supplemental S14 Video). Interestingly, the cells upstream the center do not sense any stimulus and aggregate randomly. The length of the long streams are about $4 \mathrm{~mm}$, showing that the stimulus from the centers are extended over a long distance downstream so that only the cells directly downstream of the center will show any orientation. We also used bright field microscopy to closely look at the wave propagation and streaming process under flowing buffer. Snapshots of the cell distribution during the aggregation process are shown in Fig 16. 


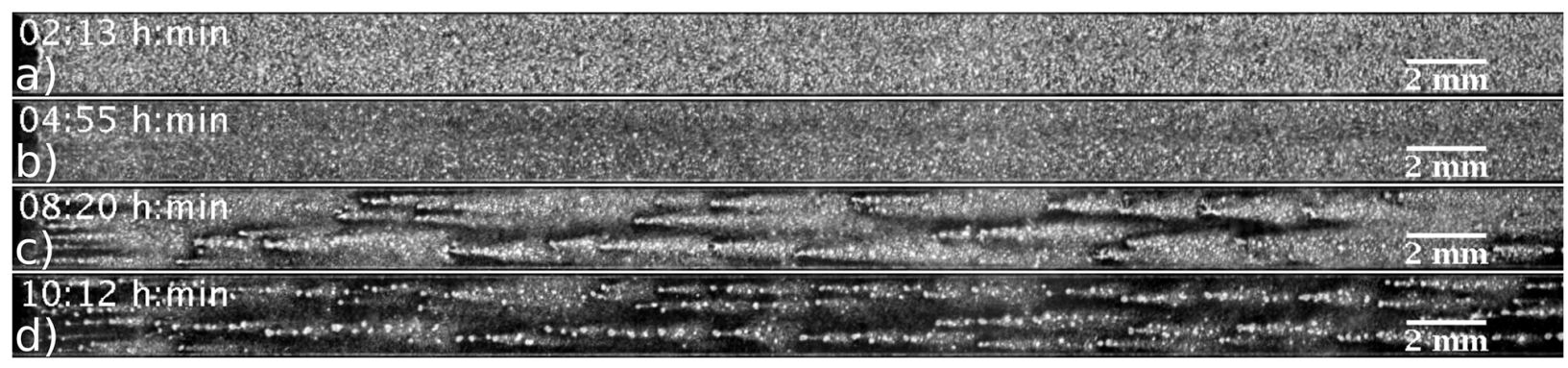

Fig 15. a) Uniform cell distribution at the beginning of experiment in a flow-through microfluidic channel $\left(V_{f}=10 \mathrm{~mm} / \mathrm{min}\right)$. b) During the propagation of the waves, the variations in cell density due to chemotactic cell movement are still negligible. c) Aggregation patterns after 8 hours starvation show cone-shaped structures with long streams downstream of the centers. d) Lateral streams, extended almost $0.5 \mathrm{~mm}$ in $y$-direction, start to line up in the direction of flow.

https://doi.org/10.1371/journal.pone.0194859.g015

In particular, the cone-shaped structures and long stream lines are well visible in Fig 16e and supplementary S15 Video.

\section{Discussion}

We have found that colonies of $D$. discoideum initiate cAMP waves even if subjected to high flow rates (see [15] for results on lower flow rates). The speed of the waves along the channel is

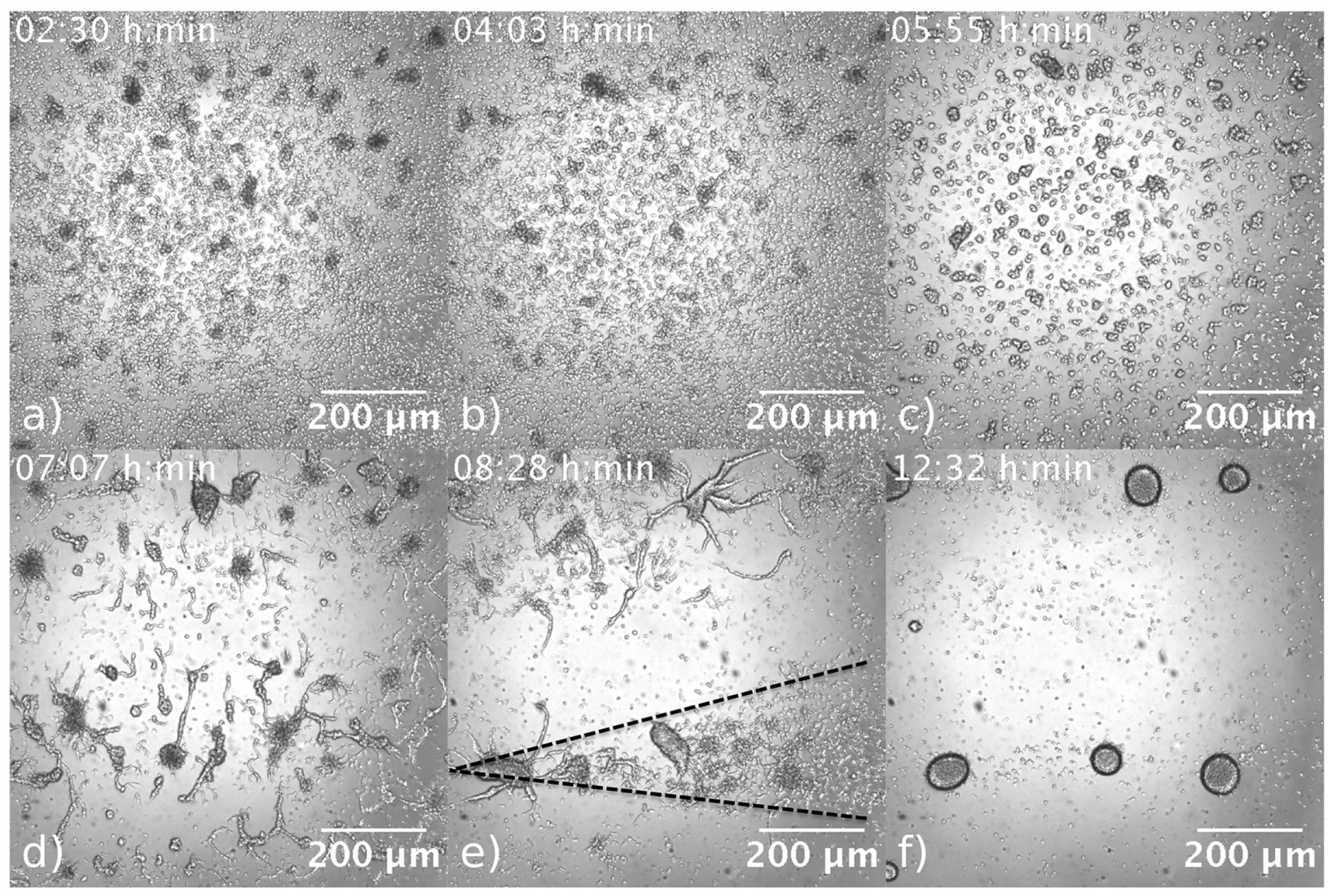

Fig 16. Aggregation process observed in a bright field microscope at $V_{f}=\mathbf{1 0} \mathrm{mm} / \mathrm{min}$. Cone-shaped aggregation domains with long stream lines are visible in panel e).

https://doi.org/10.1371/journal.pone.0194859.g016 
proportional to the imposed flow velocity, while both the velocity in the transversal direction and the wave period are independent of imposed flow velocity. The wave speed in transversal direction seems to be the same as in the spiral waves of $D$. discoideum populations in the absence of flow, where they move at $0.4 \mathrm{~mm} / \mathrm{min}$ [32] [12] and the period found is consistent with the one of target patterns in previous studies of 5-7 $\mathrm{min}$ [32] / 5-8 $\mathrm{min}$ [36]. This is a strong indicator that period and wave speed are intrinsic characteristics of the system that allow for robust aggregation even under strenuous advecting flow conditions. It is interesting that $D$. discoideum cells aggregate even at high imposed flow velocities. The type of trailing edge we observe is similar to the ones previously observed in water flow experiments of Ref. [35].

The shape of the cAMP waves changes with higher imposed flow speeds, transitioning from a planar wave to a parabolic-shaped wave that becomes increasingly longer the higher the flow rate. At high buffer flow rates, the waves are extremely elongated and their observable portion moves mostly perpendicular to the flow. Indeed, we have observed waves that are extended over the entire length of the channel. These waves, just like parabolic-shaped waves, originate at the inlet either the top or the bottom of the channel. These observations are consistent with what we observed in simulations with a developmental path, where clusters of cells advanced on the path would fail to produce a wave unless they are located near the upstream boundary. If the waves are generated upstream bottom (or top) then the cAMP quickly gets advected downstream on a time scale that is much shorter than its degradation time, thus looking like a line of cAMP that moves upwards (downwards) at the usual wave propagation velocity $(\approx 0.4$ $\mathrm{mm} / \mathrm{min})$.

We also observed an increase of the wave intensity and width in the presence of flow both in experiments and simulations. We found wave widths of up to $3 \mathrm{~mm}$, which are wider than the width of spiral waves in the undisturbed system, that have been reported around $0.3-1 \mathrm{~mm}$ [12] for the whole wave, and $0.7 \mathrm{~mm}$ at half height [32]. We understand the increase in width based on previous research showing that D.discoideum emits cAMP during a period of time of approximately $t_{f} \approx 3-4 \mathrm{~min}$ [37] / 1-3 min [32], thus in a first order approximation $d=v_{\|} \cdot t_{f}$ where $d$ is wave thickness, $v_{\|}$wave propagation velocity along the flow, and $t_{f}$ firing time. In other words, due to the flow, the cAMP produced covers a larger distance before the cell gets inactive. The increase in intensity when subjected to advection has also been observed in other reaction-diffusion systems, such as the Beluosov-Zhabotinsky reaction [38], while the increase in thickness has also been reported in numerical simulations of the FitzHugh-Nagumo model [39] and of autocatalytic fronts [40].

The wave initiation process is the most intriguing of the observed effects. We observed that for slow flow velocities the waves can start from anywhere in the system. For faster flows, we believe that the cAMP emitted by the centers is quickly advected away, and therefore do not create a supra-threshold perturbation in neighboring cells that would allow wave propagation. In the experiments, wave formation might be due to unbounded phosphodiesterase, since cells downstream would receive the enzyme secreted by the cells upstream, while the cells at the upper boundary receive a clean flow coming from the injected flow. Since this flow has an effective lower degradation, it allows for the creation of new wave pulses. More experiments using PDE deficient cells are necessary to confirm this hypothesis.

We tested the dynamical state of the cells by abruptly setting the flow velocity to zero and observing the response of the waves. In several experiments we find that the waves traveling along the channel move along it without flow as well. The waves moved at the speed of the imposed flow while the flow was applied and immediately slowed down to approximately the propagation velocity of spiral waves, as the flow was switched off. Furthermore, the width of the waves decreased, as the flow was switched off. This type of response is expected 
in excitable systems where a pulse has defined characteristics like speed and width given by the system to which the wave would return in the absence of flow. In contrast, in an oscillatory system a thicker wave would produce a synchronized (bulk) oscillation in that area, so even though the wave propagating would have a normal thickness, the area perturbed at the moment of the switch off would have a less organized behavior with areas of synchronized oscillations. We reproduced these results by switching off the flow in numerical simulations with developmental path. Even though the results depended weakly on at which point along the path the flow was stopped, they showed waves continuing to travel along the channel and some more disordered waves given by the oscillatory patches. Therefore a mixture of oscillatory and excitable cells managed to reproduce our experimental observations.

To summarize, we find that $D$. discoideum cells initiate defined cAMP waves even under the influence of strong advective flows up to $V_{f}=50 \mathrm{~mm} / \mathrm{min}$. We find that the waves change shape depending on the applied flow velocity, transitioning from planar waves at low speeds, to parabolic shaped waves, whose elongation increases with flow velocity. These wave shapes observed at high speeds were strongly dependent on the location of their initiation point. Since the cells move against cAMP gradients when aggregating, the shape of the cAMP waves have an important role on the aggregation process, in regards to this, it is noteworthy that even without the presence of wave centers such as target centers and spirals, the cells are still capable of aggregation. This also shows the capability of $D$. discoideum to signal even in extremely adverse conditions.

The wave speed in transversal direction $v_{\perp}$ and the period of the waves $T$ showed to be robust system characteristics, being constant for all studied flow speeds. The wave speed along the channel $v_{\|}$and the wave width $d$, however, scale linearly with $V_{f}$. We compare these experimental results to numerical simulations of the system. While a two-component model was sufficient for low flow velocities, we find the extension to the three-component model necessary to reproduce the wave shape at higher flows, showing how fundamental the intracellular dynamic is to produce robust signaling.

Experiments conducted with switching off the advecting flow showed waves that travel along the system changing their velocity and width to recover their unperturbed characteristics, displaying a trigger wave behavior, characteristic of excitable systems.

The observed wave shapes were successfully reproduced using a developmental path which added desynchronization to the system, having some cells in an oscillatory state and some in an excitable one. The upstream cells more advanced in this path became the source of the downstream traveling waves. This scheme also reproduced successfully the flow switching off experiments. We expect our investigations to be crucial to understand signaling of D. discoideum cells in the presence of external flows.

\section{Supporting information}

\section{S1 File. Shear stress calculations.} (PDF)

S1 Fig. Flow profile in the channel. Laminar flow profile inside the microfluidic channel in arbitrary units. a) Cut along the channel center $(y=0)$. b) Cut along half channel height $(z=0)$. (EPS)

S1 Video. Experiment without flow. The movie shows an experiment in the macro-channel without an imposed flow, so at $V_{f}=0$. The three rows of this and later movies show in order: 
i) the original Dark-field images ii) the subtracted and band-pass filtered images and iii) the local phase extracted from the band-pass filtered images.

(MOV)

S2 Video. Experiment with flow. Experiment with a low imposed flow of $V_{f}=0.5 \mathrm{~mm} / \mathrm{min}$ showing a planar wave train.

(MOV)

S3 Video. Experiment with flow. Experiment with an imposed flow of $V_{f}=5 \mathrm{~mm} / \mathrm{min}$ showing parabolic waves.

(MOV)

S4 Video. Experiment with flow. Experiment with an imposed flow of $V_{f}=10 \mathrm{~mm} / \mathrm{min}$ showing strongly elongated parabolic waves.

(MOV)

S5 Video. Experiment with flow. Experiment with an imposed flow of $V_{f}=15 \mathrm{~mm} / \mathrm{min}$ showing extremely elongated parabolic waves.

(MOV)

S6 Video. Simulations. Numerical simulation of the two-component model in the excitable regime with an imposed flow of $V_{f}=10 \mathrm{~mm} / \mathrm{min}$. Initial perturbation applied center upstream.

(MOV)

S7 Video. Simulations. Numerical simulation of the three-component model in the excitable regime with an imposed flow of $V_{f}=10 \mathrm{~mm} / \mathrm{min}$. Initial perturbation applied center upstream.

(MOV)

S8 Video. Simulations. Numerical simulation of the three-component model using a developmental path for the parameters $\sigma$ and $k_{e}$, with an imposed flow of $V_{f}=15 \mathrm{~mm} / \mathrm{min}$. Top: cAMP concentration. Bottom: State of the cells, gray for the stable state, purple for the oscillatory regime, and excitable regime in orange.

(MOV)

S9 Video. Simulations. Numerical simulation of the three-component model using a developmental path for the parameters $\sigma$ and $k_{e}$, with an imposed flow of $V_{f}=15 \mathrm{~mm} / \mathrm{min}$. Top: cAMP concentration. Bottom: State of the cells, gray for the stable state, purple for the oscillatory regime, and excitable regime in orange. (MOV)

S10 Video. On-off flow experiment. Experiment which had no flow initially, at $t_{1}=4 \mathrm{~h} 27$ min a flow of $V_{f}=1 \mathrm{~mm} / \mathrm{min}$ was switched on. The flow was switched off again at $t_{3}=5 \mathrm{~h} 21$ $\min$.

(MOV)

S11 Video. Simulations. Numerical simulation of the three-component model using a developmental path for the parameters $\sigma$ and $k_{e}$, with an imposed flow of $V_{f}=1 \mathrm{~mm} / \mathrm{min}$ until $t=250 \mathrm{~min}$, afterwards the flow is off, $V_{f}=0 \mathrm{~mm} / \mathrm{min}$. Top: cAMP concentration. Bottom: State of the cells, gray for the stable state, purple for the oscillatory regime, and excitable regime in orange.

(MOV) 
S12 Video. Jump-in-flow experiment. Experiment which had no flow initially. At $3 \mathrm{~h} 46 \mathrm{~min}$ a flow of $V_{f}=1 \mathrm{~mm} / \mathrm{min}$ was switched on. Next the flow was increased first to $V_{f}=2 \mathrm{~mm} / \mathrm{min}$ at $4 \mathrm{~h} 09 \mathrm{~min}$, then to $V_{f}=3 \mathrm{~mm} / \mathrm{min}$ at $4 \mathrm{~h} 24 \mathrm{~min}$. Finally, the speed was increased to $V_{f}=4$ $\mathrm{mm} / \mathrm{min}$ at $4 \mathrm{~h} 48 \mathrm{~min}$. At $5 \mathrm{~h} 51 \mathrm{~min}$ the flow was reduced to $V_{f}=1 \mathrm{~mm} / \mathrm{min}$ again.

$(\mathrm{MOV})$

S13 Video. Simulations. Numerical simulation of the three-component model using a developmental path for the parameters $\sigma$ and $k_{e}$, with an imposed flow of $V_{f}=1 \mathrm{~mm} / \mathrm{min}$ until $t=220 \mathrm{~min}$, afterwards the flow is increased to $V_{f}=2 \mathrm{~mm} / \mathrm{min}$ and at $t=250 \mathrm{~min}$ increased to $V_{f}=3 \mathrm{~mm} / \mathrm{min}$. Top: cAMP concentration. Bottom: State of the cells, gray for the stable state, purple for the oscillatory regime, and excitable regime in orange.

(MOV)

S14 Video. Aggregation experiment. Experiment with $V_{f}=10 \mathrm{~mm} / \mathrm{min}$ showing original Dark-field images for a longer time to show the aggregation phase of the cells.

(MOV)

S15 Video. Bright-field experiment. Bright-field microscopy experiment showing the aggregation in a channel with $V_{f}=10 \mathrm{~mm} / \mathrm{min}$ (4X magnification).

$(\mathrm{MOV})$

\section{Acknowledgments}

The authors thank M. S. Müller and K. Gunkel for their cheerful help with the preparation of the cells. T. E. acknowledges Deutsche Forschungsgemeinschaft (DFG), project number GH 184/1-1. E.V.H. thanks the Deutsche Akademische Austauschdienst (DAAD), Research Grants-Doctoral Programs in Germany. A.G. acknowledges MaxSynBio Consortium, which is jointly funded by the Federal Ministry of Education and Research of Germany and the Max Planck Society.

\section{Author Contributions}

Formal analysis: Torsten Eckstein, Estefania Vidal-Henriquez.

Investigation: Torsten Eckstein, Estefania Vidal-Henriquez, Albert Bae, Vladimir Zykov, Eberhard Bodenschatz, Azam Gholami.

Supervision: Azam Gholami.

Writing - original draft: Torsten Eckstein, Estefania Vidal-Henriquez, Azam Gholami.

Writing - review \& editing: Torsten Eckstein, Estefania Vidal-Henriquez, Albert Bae, Vladimir Zykov, Eberhard Bodenschatz, Azam Gholami.

\section{References}

1. Rovinsky AB, Menzinger M. Chemical instability induced by a differential flow. Physical Review Letters. 1992; 69(8):1193. https://doi.org/10.1103/PhysRevLett.69.1193 PMID: 10047151

2. Rovinsky $A B$, Menzinger $M$. Self-organization induced by the differential flow of activator and inhibitor. Physical Review Letters. 1993; 70(6):778. https://doi.org/10.1103/PhysRevLett.70.778 PMID: 10054201

3. Wu XG, Nakata S, Menzinger M, Rovinsky A. Differential flow instability in tubular flow reactor: Its convective nature. The Journal of Physical Chemistry. 1996; 100(39):15810-15814. https://doi.org/10. 1021/jp9603546

4. Turing AM. The chemical basis of morphogenesis. Philosophical Transactions of the Royal Society of London B: Biological Sciences. 1952; 237(641):37-72. https://doi.org/10.1098/rstb.1952.0012 
5. Malchow $\mathrm{H}$. Flow-and locomotion-induced pattern formation in nonlinear population dynamics. Ecological Modelling. 1995; 82(3):257-264. https://doi.org/10.1016/0304-3800(94)00095-Y

6. Malchow H. Motion instabilities in prey-predator systems. Journal of Theoretical Biology. 2000; 204:639-647. https://doi.org/10.1006/jtbi.2000.2074 PMID: 10833362

7. Malchow H. Non-equilibrium spatio-temporal patterns in models of non-linear plankton dynamics. Freshwater Biology. 2000; 45(2):239-251. https://doi.org/10.1046/j.1365-2427.2000.00550.x

8. Scheffer M. Should we expect strange attractors behind plankton dynamics-and if so, should we bother? Journal of Plankton Research. 1991; 13(6):1291-1305.

9. Kærn M, Menzinger M, Satnoianu R, Hunding A. Chemical waves in open flows of active media: their relevance to axial segmentation in biology. Faraday discussions. 2002; 120:295-312. https://doi.org/ 10.1039/b103244p

10. Pálsson E, Cox EC. Origin and evolution of circular waves and spirals in Dictyostelium discoideum territories. Proceedings of the National Academy of Sciences. 1996; 93(3):1151-1155.

11. Lee KJ, Cox EC, Goldstein RE. Competing Patterns of Signaling Activity in Dictyostelium Discoideum Phys Rev Lett. 1996; 76:1174-1177. https://doi.org/10.1103/PhysRevLett.76.1174 PMID: 10061652

12. Tomchik K, Devreotes P. Adenosine 3',5'-monophosphate waves in Dictyostelium discoideum: a demonstration by isotope dilution-fluorography. Science. 1981; 212(4493):443-446. https://doi.org/10. 1126/science.6259734 PMID: 6259734

13. Kessler DA, Levine H. Pattern formation in Dictyostelium via the dynamics of cooperative biological entities. Phys Rev E. 1993; 48:4801-4804. https://doi.org/10.1103/PhysRevE.48.4801

14. Mosley MP. Subsurface flow velocities through selected forest soils, South Island, New Zealand. Journal of hydrology. 1982; 55(1-4):65-92. https://doi.org/10.1016/0022-1694(82)90121-4

15. Gholami A, Steinbock O, Zykov V, Bodenschatz E. Flow-Driven Waves and Phase-Locked Self-Organization in Quasi-One-Dimensional Colonies of Dictyostelium discoideum. Physical review letters. 2015; 114(1):018103. https://doi.org/10.1103/PhysRevLett.114.018103 PMID: 25615506

16. Gholami A, Steinbock $O$, Zykov V, Bodenschatz E. Flow-driven instabilities during pattern formation of Dictyostelium discoideum. New Journal of Physics. 2015; 17(6):063007. https://doi.org/10.1088/13672630/17/6/063007

17. Gholami A, Zykov V, Steinbock $O$, Bodenschatz E. Flow-driven two-dimensional waves in colonies of Dictyostelium discoideum. New Journal of Physics. 2015; 17(9):093040. https://doi.org/10.1088/13672630/17/9/093040

18. Martiel JL, Goldbeter A. A model based on receptor desensitization for cyclic AMP signaling in Dictyostelium cells. Biophysical journal. 1987; 52(5):807. https://doi.org/10.1016/S0006-3495(87)83275-7 PMID: 19431710

19. Lauzeral J, Halloy J, Goldbeter A. Desynchronization of cells on the developmental path triggers the formation of spiral waves of cAMP during Dictyostelium aggregation. Proceedings of the National Academy of Sciences. 1997; 94(17):9153-9158. https://doi.org/10.1073/pnas.94.17.9153

20. Parent CA, Devreotes PN. Molecular Genetics of Signal Transduction in Dictyostelium. Annual Review of Biochemistry. 1996; 65(1):411-440. https://doi.org/10.1146/annurev.bi.65.070196.002211 PMID: 8811185

21. Whitesides GM, Ostuni E, Takayama S, Jiang X, Ingber DE. Soft lithography in biology and biochemistry. Annual review of biomedical engineering. 2001;3(1):335-373. https://doi.org/10.1146/annurev. bioeng.3.1.335 PMID: 11447067

22. Décavé E, Rieu D, Dalous J, Fache S, Bréchet Y, Fourcade B, et al. Shear flow-induced motility of Dictyostelium discoideum cells on solid substrate. Journal of cell science. 2003; 116(21):4331-4343. https://doi.org/10.1242/jcs.00726 PMID: 12966168

23. Edelstein AD, Tsuchida MA, Amodaj N, Pinkard H, Vale RD, Stuurman N. Advanced methods of microscope control using $\mu$ Manager software. Journal of biological methods. 2014; 1(2). https://doi.org/10. 14440/jbm.2014.36 PMID: 25606571

24. Siegert F, Weijer CJ. Spiral and concentric waves organize multicellular Dictyostelium mounds. Current Biology. 1995; 5(8):937-943. https://doi.org/10.1016/S0960-9822(95)00184-9 PMID: 7583152

25. Egolf DA, Melnikov IV, Bodenschatz E. Importance of local pattern properties in spiral defect chaos. Physical Review Letters. 1998; 80(15):3228. https://doi.org/10.1103/PhysRevLett.80.3228

26. Tyson JJ, Alexander KA, Manoranjan V, Murray J. Spiral waves of cyclic AMP in a model of slime mold aggregation. Physica D: Nonlinear Phenomena. 1989; 34(1-2):193-207. https://doi.org/10.1016/01672789(89)90234-0

27. Merson R. An operational method for the study of integration processes. In: Proc. Symp. Data Processing; 1957. p. 1-25. 
28. Hundsdorfer W, Verwer JG. Numerical solution of time-dependent advection-diffusion-reaction equations. vol. 33. Springer Science \& Business Media; 2013.

29. Vidal-Henriquez E, Zykov V, Bodenschatz E, Gholami A. Convective instability and boundary driven oscillations in a reaction-diffusion-advection model. Chaos: An Interdisciplinary Journal of Nonlinear Science. 2017; 27(10):103110. https://doi.org/10.1063/1.4986153

30. Alcantara F, Monk M. Signal propagation during aggregation in the slime mould Dictyostelium discoideum. Microbiology. 1974; 85(2):321-334.

31. Gross J, Peacey M, Trevan D. Signal emission and signal propagation during early aggregation in Dictyostelium discoideum. Journal of cell science. 1976; 22(3):645-656. PMID: 1035221

32. Devreotes PN, Potel MJ, MacKay SA. Quantitative analysis of cyclic AMP waves mediating aggregation in Dictyostelium discoideum. Developmental Biology. 1983; 96(2):405-415. https://doi.org/10.1016/ 0012-1606(83)90178-1 PMID: 6299820

33. Geberth D, Hütt MT. Predicting the distribution of spiral waves from cell properties in a developmentalpath model of Dictyostelium pattern formation. PLoS computational biology. 2009; 5(7):e1000422. https://doi.org/10.1371/journal.pcbi.1000422 PMID: 19593362

34. Wessels D, Brincks R, Kuhl S, Stepanovic V, Daniels KJ, Weeks G, et al. RasC plays a role in transduction of temporal gradient information in the cyclic-AMP wave of Dictyostelium discoideum. Eukaryotic Cell. 2004; 3(3):646-662. https://doi.org/10.1128/EC.3.3.646-662.2004 PMID: 15189986

35. Bonner JT, Savage LJ. Evidence for the formation of cell aggregates by chemotaxis in the development of the slime mold Dictyostelium discoideum. Journal of Experimental Zoology. 1947; 106(1):1-26. https://doi.org/10.1002/jez.1401060102 PMID: 20268085

36. Siegert F, Weijer C. Digital image processing of optical density wave propagation in Dictyostelium discoideum and analysis of the effects of caffeine and ammonia. Journal of Cell Science. 1989; 93(2):325335.

37. Van Haastert PJ. A method for studying cAMP-relay in Dictyostelium discoideum: the effect of temperature on cAMP-relay. Microbiology. 1984; 130(10):2559-2564. https://doi.org/10.1099/00221287-13010-2559

38. Kærn M, Menzinger M. Propagation of excitation pulses and autocatalytic fronts in packed-bed reactors The Journal of Physical Chemistry B. 2002; 106(14):3751-3758. https://doi.org/10.1021/jp013541z

39. Ermakova EA, Shnol EE, Panteleev MA, Butylin AA, Volpert V, Ataullakhanov FI. On propagation of excitation waves in moving media: The FitzHugh-Nagumo model. PloS One. 2009; 4(2):e4454. https:// doi.org/10.1371/journal.pone.0004454 PMID: 19212435

40. Leconte M, Martin J, Rakotomalala N, Salin D, Yortsos Y. Mixing and reaction fronts in laminar flows. The Journal of chemical physics. 2004; 120(16):7314-7321. https://doi.org/10.1063/1.1665555 PMID: 15267641 


\section{Shear stress}

To calculate the shear stress acting on the cells in the microfluidic channel, we solve the incompressible Navier-Stokes equation in 3D. The channel has a rectangular geometry $(x \in[0, L], y \in[-c, c], z \in[-b, b])$ and we use zero velocity boundary conditions at the boundaries of the channel cross section $(u(y= \pm c)=0$ and $u(z= \pm b)=0)$,

$$
\rho \frac{D u}{D t}=\rho g-\nabla p+\mu \nabla^{2} u
$$

Here $\mu$ denotes fluid viscosity, $\rho$ fluid density, $p$ pressure, and $u$ fluid velocity. We solve using variable separation and Fourier series, and find an expression for the fluid velocity $u$

$$
\begin{aligned}
u & =\frac{G\left(b^{2}-z^{2}\right)}{2 \mu}+\sum_{n \text { odd }} A_{n} \cosh \left(\frac{n \pi}{2 b} y\right) \cos \left(\frac{n \pi}{2 b} z\right), \quad \text { with } \\
A_{n} & =-\frac{16 G b^{2}}{\mu \pi^{3} n^{3}} \frac{\sin \left(\frac{n \pi}{2}\right)}{\cosh \left(\frac{n \pi c}{2 b}\right)} .
\end{aligned}
$$

Where $G$ denotes the pressure drop in the channel. For a detailed derivation of the velocity please see [1].

We now calculate the wall shear stress from the velocity profile, using the expression

$$
\tau=\left.\mu \partial_{z} u\right|_{z=-c}
$$

and the velocity space derivative

$$
\partial_{z} u=-\frac{G z}{\mu}-\frac{n \pi}{2 b} \sum_{n \text { odd }} A_{n} \cosh \left(\frac{n \pi}{2 b} y\right) \sin \left(\frac{n \pi}{2 b} z\right) .
$$

Considering we do not have access to the pressure drop $G$ across the channel, but only the mean flow $Q$ we find an expression for the mean flow by integrating over the channel cross section,

$$
Q=\frac{G b^{3} c}{\mu}\left[\frac{4}{3}-\frac{8 b}{c} \sum_{n \text { odd }}\left(\frac{2}{n \pi}\right)^{5} \tanh \left(\frac{n \pi c}{2 b}\right)\right],
$$

thus we obtain the pressure drop $G$

$$
G=\frac{Q \mu}{b^{3} c}\left[\frac{4}{3}-\frac{8 b}{c} \sum_{n \text { odd }}\left(\frac{2}{n \pi}\right)^{5} \tanh \left(\frac{n \pi c}{2 b}\right)\right]^{-1} .
$$

We can simplify $G$ by using the values for $c$ and $b$. Note also that since $\pi c /(2 b) \approx 31.4$ the hyperbolic tangent becomes $\tanh (\pi c /(2 b)) \approx 1$, this leads to

$$
\begin{aligned}
G & =\frac{Q \mu}{b^{3} c} \times\left[\frac{4}{3}-\epsilon\right]^{-1} \quad \text { with } \\
\epsilon & =\frac{8 b}{c} \sum_{n \text { odd }}\left(\frac{2}{n \pi}\right)^{5} \tanh \left(\frac{n \pi c}{2 b}\right) \approx 4.2 \times 10^{-2} .
\end{aligned}
$$




\section{snow -}

\begin{tabular}{l|c}
$V_{f}\left[\frac{\mathrm{mm}}{\min }\right]$ & $\tau[\mathrm{Pa}]$ \\
\hline 1 & 0.000918 \\
5 & 0.004590 \\
10 & 0.009180 \\
15 & 0.013771 \\
20 & 0.018361 \\
30 & 0.027541 \\
50 & 0.045902
\end{tabular}

Table 1. Shear stress $\tau$ calculated as a function of imposed flow velocity $V_{f}$, using $\mu=8.9 \cdot 10^{-4} \mathrm{~Pa} \mathrm{~s}$, the dynamic viscosity of water and the channel dimensions $c=1$ $\mathrm{mm}$ and $b=0.05 \mathrm{~mm}$.

Then we can approximate this for small $\epsilon$

$$
G \approx \frac{Q \mu}{b^{3} c}\left[\frac{3}{4}+\left(\frac{3}{4}\right)^{2} \epsilon\right] .
$$

Since we want to find the maximum stress, we choose $y$ to be at the middle of the channel $(y=0)$. Thus, we can simplify $\tau$ as well, $\operatorname{since} \cosh (n \pi y / 2 b)=1$, and $A_{n} \approx 0$ $\forall n \in \mathbb{N}$,

$$
\tau_{y=0}=-G z=-\frac{Q \mu z}{b^{3} c} \times\left[\frac{3}{4}+\left(\frac{3}{4}\right)^{2} \epsilon\right] .
$$

We can now calculate the shear stress for the imposed mean flow velocities used in our experiments. An overview is shown in Table 1.

Thus, the shear stresses are well below the critical value for the onset of mechanosensivity $0.7 \mathrm{~Pa}$ or even detachment 2.6 $\mathrm{Pa}[2]$.

\section{References}

1. Vidal-Henriquez E, Zykov V, Bodenschatz E, Gholami A. Convective instability and boundary driven oscillations in a reaction-diffusion-advection model. Chaos: An Interdisciplinary Journal of Nonlinear Science. 2017;27(10):103110. doi:10.1063/1.4986153.

2. Décavé E, Rieu D, Dalous J, Fache S, Bréchet Y, Fourcade B, et al. Shear flow-induced motility of Dictyostelium discoideum cells on solid substrate. Journal of cell science. 2003;116(21):4331-4343. doi:10.1242/jcs.00726. 
Chapter 2 - Influence of fast advective flows on pattern formation of Dictyostelium discoideum
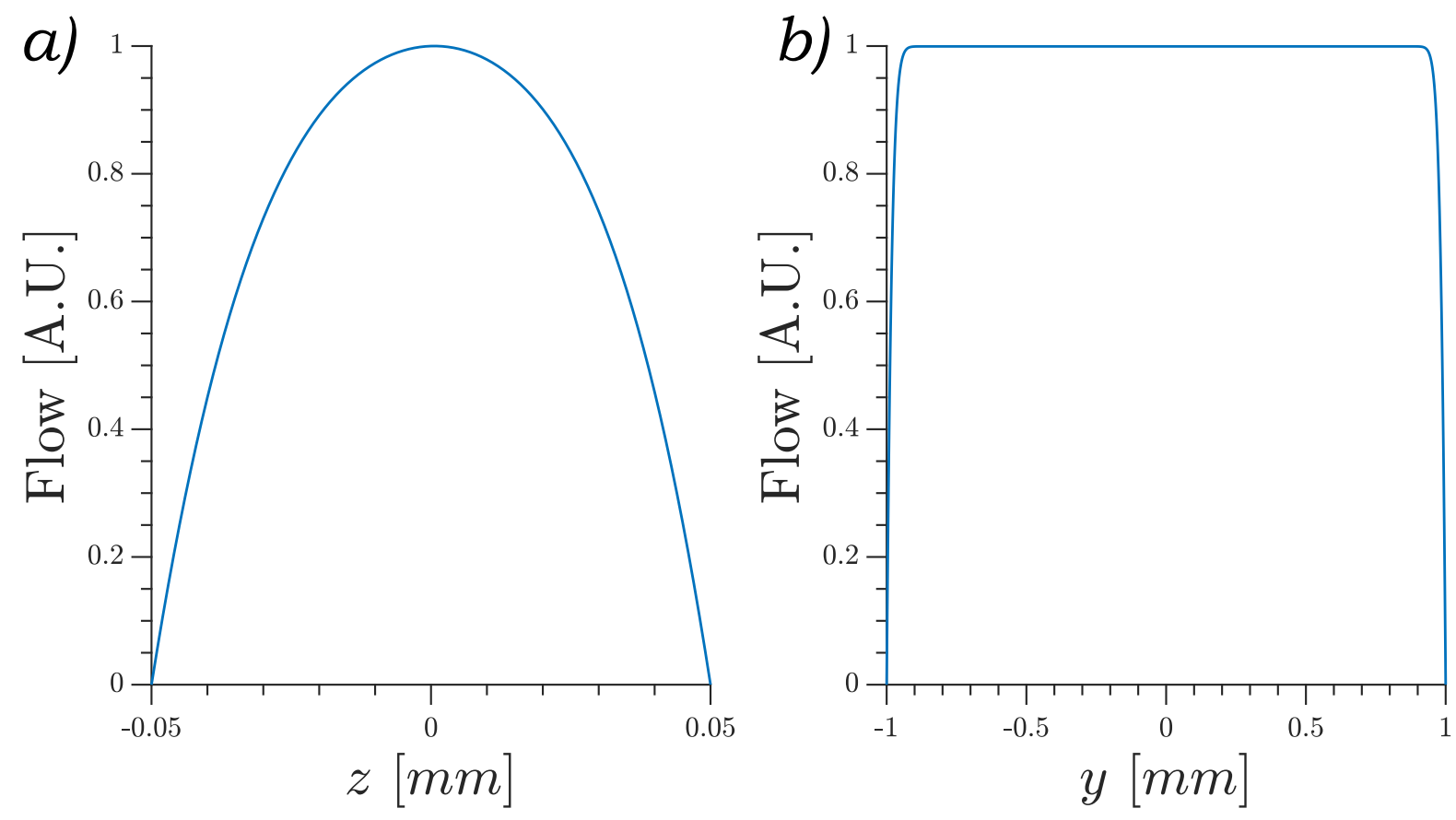

Figure 2.1: Laminar flow profile inside the microfluidic channel in arbitrary units. a) Cut along the channel center $(\mathrm{y}=0)$. b) Cut along half channel height $(\mathrm{z}=$ $0)$. 


\section{Experimental observation of boundary-driven oscillations in a reaction-diffusion-advection system}

The following paper draft is the base for a manuscript that has since been accepted by Soft matter as Eckstein T, Vidal-Henriquez E and Gholami A. Experimental observation of boundary-driven oscillations in a reaction-diffusion-advection system. Soft Matter, 2020, DOI: 10.1039/C9SM02291K.

TE performed experiments. All authors analyzed data. All authors wrote the manuscript. TE produced figures 1, 2, 3, 4, 5, 6, 7, 8, 9 and 10 . 


\title{
Soft Matter
}

\section{ARTICLE TYPE}

Cite this: DOI: 00.0000/xxxxxxxxxx

\section{Experimental observation of boundary-driven oscillations in a reaction-diffusion-advection system ${ }^{\dagger}$}

\author{
Torsten Eckstein, ${ }^{\ddagger}$ Estefania Vidal-Henriquez, ${ }^{\ddagger}$ and Azam Gholami
}

Received Date

Accepted Date

DOI: 00.0000/xxxxxxxxxx
Boundary-driven oscillations were numerically predicted to exist in a reaction-diffusion-advection system, namely in signaling population of social amoeba $D$. discoideum ${ }^{1}$. If deprived of nutrients, $D$. discoideum aggregate by producing CAMP waves at precisely timed intervals. In the presence of an advecting flow, holding the upstream boundary to a zero concentration of CAMP produces an instability that sends periodically wave trains downstream. This instability is expected to exist at lower degradation rates of cAMP and thus provides a mechanism for wave creation in phosphodiesterase deficient systems, such as PdsA- cells. Degradation of extracellular cAMP by the enzyme phosphodiesterase PdsA is fundamental to successfully producing waves, regulating the external cAMP gradient field and preventing the accumulation of cAMP. Using a flow-through microfluidic set up filled with $\mathrm{PdsA}^{-}$cells, we confirm experimentally that boundary-driven oscillations indeed exist. Above a minimum flow velocity, decaying waves are induced, with a decay length that increases with the imposed flow velocity. We performed extensive numerical simulations and showed that these waves have a boundary-driven origin, where the lack of cAMP in the upstream flow destabilizes the system. We explored the properties of these waves and the parameter region where they exist, with good agreement with our experimental observations. These results provide experimental confirmation of the destabilizing effect of the upstream boundary in an otherwise stable reaction-diffusion system. We expect this mechanism to be relevant for wave creation in other oscillatory or excitable systems that are incapable of wave generation in the absence of flow.

\section{Introduction}

The social amoeba Dictyostelium discoideum (D.discoideum) is a paradigm model organism to study biological pattern formation and provides insights into the nature of cell-cell communication and emergent collective behavior ${ }^{2-5}$. D. discoideum cells lead a solitary life as long as the food supply is sufficient. Upon nutrient depletion the cells start an aggregation process in which they gather in groups of around $10^{4}-10^{6}$ amoebas and form multicellular structures known as fruiting bodies in order to survive 6,7 . This aggregation process is governed by the chemotactic response of $D$. discoideum cells to outward propagating large-scale cAMP (cyclic adenosine monophosphate) waves that trigger oriented periodic movement of the cells towards the aggregation centers. These waves are generated by an interplay between random diffusion of cAMP in the extracellular medium and the ability of the cells both to receive the signal via cAMP receptors on the membrane of individual cells as well as to relay and amplify the signal

Max Planck Institute for Dynamics and Self-Organization, Göttingen, Germany. E-mail: azam.gholami@ds.mpg.de

$\dagger$ Electronic Supplementary Information (ESI) available: See DOI: $00.0000 / 00000000$.

$\ddagger$ These authors contributed equally to this work by producing more cAMP. The cells crawl up the concentration gradient for a few minutes until the chemotactic response of the cells is adapted ${ }^{8}$.

The limiting factor in signal amplification is the desensitization of cAMP receptors if they are persistently exposed to high concentrations of extracellular cAMP ${ }^{9-11}$. To avoid receptor desensitization and to produce strong cAMP gradients, the cells emit phosphodiesterases (PDEs) that degrade the extracellular cAMP. Three extracellular PDEs have been characterized ${ }^{12}$ in D. discoideum: PDE1 (also called PdsA or PdeA), PDE4, and PDE7. All three types of PDEs have different dynamics, therefore becoming more relevant for cAMP degradation during different parts of the developmental program. During the early aggregation stage, PdsA is the dominant PDE, degrading the extracellular cAMP almost all by itself. The knockout mutant PdsA- does not produce PdsA and has been shown to be unable to produce cAMP waves, and thus fail to aggregate ${ }^{13}$. Rescue of this cell type was reported by adding PDE to the cell solution, which allowed for normal aggregation $^{13}$. Moreover, it has been shown that PdsA' amoebas show oscillations in the concentration of intracellular cAMP if subjected to a fresh buffer flow that carries extracellular cAMP away ${ }^{14}$, suggesting that the external flow can replace the role of PDE.

In their natural habitat, $D$. discoideum cells are exposed to ex- 
ternal flows which can significantly influence the wave generation process ${ }^{15}$. Previously, we had numerically predicted the existence of a convective instability induced by the influx of cAMPfree buffer through a colony of signaling amoebas ${ }^{1,16}$. In such a system it is of utmost importance what kind of chemicals get injected in the system with the advecting flow. In the numerical simulations this is equivalent to what boundary conditions are used in the upstream edge of the system. In the case where there are no cells upstream, the flow would be free of cAMP and can act as a destabilizing agent. We have shown that holding the upstream boundary to a zero concentration of cAMP produces an instability that periodically sends wave trains downstream. The wave generation mechanism works by first advecting the cAMP downstream, thus depleting the upstream area of cAMP. This low concentration of the chemoattractant destabilizes the cells close to the upstream boundary which react by releasing a pulse of cAMP. This instability, known as boundary-driven oscillations ${ }^{1}$, exists at lower degradation rates than the oscillatory regime and thus provides a mechanism for wave creation in phosphodiesterase-deficient systems, such as PdsA- cells.

In this work, we present experimental evidence of the existence of boundary-driven oscillations (BDOs), which can be observed in a microfluidic setup filled with starving PdsA- cells. Note that the imposed flow is not strong enough to detach the cells from the substrate and the cAMP produced by the cells is advected downstream. Interestingly, at small flow velocities we observe decaying cAMP waves that do not fill the whole length of the channel, with a decay length that grows with the advecting flow velocity. Our extensive numerical simulations confirm a similar trend in the decay length of the waves as the flow is increased. We also quantified the period of these boundary-driven waves, measuring high wave periods ( $\sim 20 \mathrm{~min}$ ) at small advective flows which decrease with the imposed flow velocity, approaching the natural period of $6 \mathrm{~min}$ at flow velocities larger than $0.6 \mathrm{~mm} / \mathrm{min}$. Interestingly, once the flow is switched off, the cells located upstream of the microfluidic channel (that have experienced BDOs) continue to produce cAMP waves similar to wild type cells, but with a much larger wave period of around $12 \mathrm{~min}$. These amoebas are able to aggregate normally, rescuing the aggregation phenotype. However, cells downstream of the channel located outside the penetration depth of BDOs fail to aggregate once the flow is switched off. Thus, at high flow velocities where the BDOs penetrate the whole length of the channel, all the cells in the channel were rescued. We observed a similar effect as we used WT cells at the upstream area of the channel. The cAMP waves generated by WT cells penetrate gradually throughout the population of PdsA ${ }^{-}$cells, filling the whole length of the microfluidic channel with time.

\section{Materials and Methods}

\section{Cell Culture}

All experiments were performed with $D$. discoideum UK5 cells, denoted as PdsA- (Dicty Stock Center, Chicago, IL, USA). Cells were grown in HL-5 medium (35.5 $\mathrm{g}$ of Formedium powder from Formedium Ltd, Norfolk, England, per liter of double-distilled wa- ter, autoclaved and filtered) at $22{ }^{\circ} \mathrm{C}$ on polystyrene Petri dishes (TC Dish 100, Sarsted, Nümbrecht Germany) and harvested when they became confluent. Before the experiments, the cells were centrifuged and washed two times with phosphate buffer (2 $\mathrm{g}$ of $\mathrm{KH}_{2} \mathrm{PO}_{4}$ and $0.36 \mathrm{~g}$ of $\mathrm{Na}_{2} \mathrm{HPO}_{4} \cdot \mathrm{H}_{2} \mathrm{O}$ per liter at $\mathrm{pH}$ 6.0, autoclaved, both from Merck, Darmstadt, Germany). The cells were then resuspended in $200 \mu$ l phosphate buffer. The cell density was determined using a hemocytometer (Neubauer ZÃd'hlkammer, VWR, Darmstadt, Germany), diluted to $5 \times 10^{7}$ cells $/ \mathrm{ml}$ of phosphate buffer and injected into the microfluidic channel.

\section{Microfluidics}

The microfluidic devices were fabricated by means of standard soft lithography ${ }^{17}$ methods. A silicon wafer was coated with a $100 \mu \mathrm{m}$ photoresist layer (SU-8 100, Micro Resist Technology $\mathrm{GmbH}$, Berlin, Germany) and patterned by photolithography to obtain a structured master wafer. The channels are $2 \mathrm{~mm}$ wide, $50 \mathrm{~mm}$ long, and $103 \pm 2 \mu \mathrm{m}$ high. Polydimethylsiloxane (PDMS, 10:1 mixture with curing agent, Sylgard 184, Dow Corning $\mathrm{GmbH}$, Wiesbaden, Germany) was poured onto the wafer and cured for $1 \mathrm{~h}$ at $75^{\circ} \mathrm{C}$. To produce the microfluidic device, a PDMS block containing the macro-channels was cut out, and two inlets ( $7 \mathrm{~mm}$ and $0.75 \mathrm{~mm}$ in diameter) were punched through the PDMS at opposite ends of the channel with the help of PDMS punchers (Harris Uni-Core-7.00 and Harris Uni-Core-0.75, SigmaAldrich, St. Louis, MO, USA). Afterwards, a glass microscope slide $(76 \times 26 \mathrm{~mm}$, VWR, Darmstadt, Germany) was sealed to the PDMS block following a 20-30 s treatment in air plasma (PDC 002, Harrick Plasma, Ithaca, USA) to close the macro-channels. The large inlet (7 $\mathrm{mm}$ diameter) was used as a liquid reservoir filled with phosphate buffer and from the other side phosphate buffer was sucked out using a high precision syringe pump (PHD 2000 Infuse/Withdraw Syringe Pump from Harvard Apparatus, Holliston, USA, combined with gas-tight glass syringes from Hamilton, Reno, USA) at constant buffer flow rates.

\section{Image Acquisition and Analysis}

We used a dark-field setup consisting of a monochrome 12-bit CCD camera (QIClick-F-M-12, QImaging, Surrey, Canada), a telecentric lens (0.16X SilverTL Telecentric Lens, Edmund Optics Inc, Barrington, NJ, USA) and a ring light source (Advanced Illumination, Rochester, VT, USA). The camera was controlled with an image capture program (Micro-Manager ${ }^{18}$ ) and it recorded images every 20 seconds. We processed the dark-field videos by first subtracting the intensity trend of each pixel to normalize the signal in time. Next, we did spatial band-pass filtering to suppress structures bigger than $3.5 \mathrm{~mm}$ and smaller than $0.294 \mathrm{~mm}$. Finally, we used the Hilbert transform ${ }^{19}$ to calculate the phase of each pixel, thus obtaining the phase maps.

\section{Numerical Simulations}

The Martiel-Goldbeter model ${ }^{20}$ is a reaction-diffusion model proposed in 1987 to describe pattern formation in D. discoideum and has been successfully used to describe spiral formation ${ }^{3,21}$, wave advection $^{22}$, spontaneous center formation ${ }^{23}$, and cell stream- 
ing ${ }^{24}$. Changing the parameters in the model allows us to describe the system at different points during the starvation process or with knocked-out genes. To describe PdsA`cells, then, we use this model with a very low cAMP degradation rate.

Numerical simulations of the Martiel-Goldbeter model ${ }^{20}$ to describe cAMP production and relay in $D$. discoideum were performed and compared with experimental results. The relay process starts when the cells detect the presence of cAMP in the extracellular media through the receptors located on the cell membrane. After binding with cAMP, the receptors change to a phosphorylated or inactive state, in which they have a lower probability of binding with cAMP. After some relaxation time, the receptors go back to their active state. The binding of the receptors with cAMP triggers a series of reactions inside the cell that culminate with the production of cAMP and the cells' motion against the wave direction. The produced cAMP is degraded into AMP by the intracellular phosphodiesterase and transported passively to the extracellular media. Finally, once outside of the cells, the cAMP is free to diffuse and is degraded by the action of phosphodiesterase in the extracellular media. This is the type of phosphodiesterase that $\mathrm{PdsA}^{-}$cells do not produce.

The equations are

$$
\begin{aligned}
\partial_{t} \gamma & =k_{t} \beta / h-k_{e} \gamma+D \nabla^{2} \gamma-V_{f} \cdot \nabla \gamma \\
\partial_{t} \beta & =q \sigma \alpha \Phi(\rho, \gamma) /(1+\alpha)-\left(k_{i}+k_{t}\right) \beta, \text { and } \\
k_{1}^{-1} \partial_{t} \rho & =-f_{1}(\gamma) \rho+f_{2}(\gamma)(1-\rho),
\end{aligned}
$$

where the modeled fields are the extracellular concentration of cAMP $\gamma(x, t)$, the intracellular concentration of cAMP $\beta(x, t)$, and the percentage of active receptors on the cell membrane $\rho(x, t)$. $D$ stands for the cAMP diffusion, $V_{f}$ the velocity of the externally applied flow, $\Phi$ is the nonlinear function for cAMP production, $f_{1}$ the function for receptors phosphoryliation and $f_{2}$ for receptors resensitization, these functions are given by

$$
\begin{gathered}
f_{1}(\gamma)=\frac{1+\kappa \gamma}{1+\gamma}, \quad f_{2}(\gamma)=\frac{\mathscr{L}_{1}+\kappa \mathscr{L}_{2} c \gamma}{1+c \gamma}, \\
\Phi(\rho, \gamma)=\frac{\lambda_{1}+Y^{2}}{\lambda_{2}+Y^{2}}, \quad Y=\frac{\rho \gamma}{1+\gamma} .
\end{gathered}
$$

All used parameters are indicated in Table 1, and were kept fixed with the exception of $\sigma$ (linear on the production rate) and $k_{e}$ (degradation rate) which were used to explore the parameter space. To reproduce the signaling process in $\mathrm{PdsA}^{-}$cells, $k_{e}$ was kept low, $k_{e} \ll 1 \mathrm{~min}^{-1}$, compared to simulations of wild type cells where $k_{e} \approx 5-12 \mathrm{~min}^{-13,20,21}$.

Numerical simulations were performed using finite differences with a 3 points Laplacian in 1-D and 5 points in 2-D for the space discretization and a Runge-Kutta scheme with an adaptative time step $^{25}$ for the time evolution. A nonlinear discretization of the advection operator was used to ensure positivity of the cAMP concentration $\gamma$ following the work of Koren ${ }^{26}$. To account for the injection of buffer without cAMP an absorbing boundary condition was used, that is, the value of $\gamma$ was fixed at zero at the upstream boundary, $\gamma(x=0, t)=0$. At the downstream boundary no-flux $\left(\partial_{x} \gamma(x=L, t)=0\right)$ boundary conditions were used. For a detailed analysis of the effects of advection in this setup filled with WT cells, refer to our previous works ${ }^{1,22}$.

$$
\begin{array}{|c|c|c|}
\hline c=10 & h=5 & k_{1}=0.09 \mathrm{~min}^{-1} \\
\kappa=18.5 & \alpha=3 & k_{i}=1.7 \mathrm{~min}^{-1} \\
k_{t}=0.9 \mathrm{~min}^{-1} & \mathscr{L}_{1}=10 & \mathscr{L}_{2}=0.005 \\
q=4000 & \lambda_{1}=10^{-4} & \lambda_{2}=0.2575 \\
& D=0.024 \mathrm{~mm}^{2} / \min & \\
\hline
\end{array}
$$

Table 1 Parameters used in numerical simulations of Equation 1

\section{Results}

\section{A fresh cAMP-free buffer flow can substitute PdsA ${ }^{-}$activity}

In a population of starving $D$. discoideum cells, the level of extracellular cAMP is controlled by the activity of the enzyme adenylyl cyclase (ACA), which catalyzes the reaction in which cAMP is produced, and phosphodiestrase, which degrades cAMP. It is believed that PdsA, by degrading extracellular cAMP, plays an important role in regulating chemotaxis by establishing cAMP gradients during the streaming process. Upon starvation, $\mathrm{PdsA}^{-}$cells fail to produce cAMP waves and thus can not aggregate. However, in our experiments with flow-through microfluidic channels, we observe that an external flow can effectively play the role of PdsA by removing the extracellular cAMP. Thereby, cAMP waves are recovered and the aggregation process is rescued.

We observe boundary-driven waves in a population of PdsA' cells if they experience the external flow for at least three hours. The minimum imposed flow velocity needed to recover cAMP waves in our experiments was $V_{f} \geq 0.3 \mathrm{~mm} / \mathrm{min}$. At velocities below $0.3 \mathrm{~mm} / \mathrm{min}$ we did not observe any waves even during our long-run experiments where buffer was flowing through the channel for more than 10 hours. Figure 1a-c shows examples of boundary-driven waves at low, moderate and high imposed flow velocities, respectively. In all of our experiments, the cAMP waves develop within three hours of imposing the flow. While the waves at $V_{f}=0.3 \mathrm{~mm} / \mathrm{min}$ decay quickly as they initiate at the upstream end of the channel and never successfully propagate throughout the channel, waves at $V_{f}=0.7 \mathrm{~mm} / \mathrm{min}$ eventually are able to penetrate and travel along the whole length of the channel. Full penetration of the waves in the channel at moderate flow speeds, occurs within the time scale of three hours and the transition from partial to full penetration is fairly sharp. Interestingly, at high flow speed of $V_{f}=1.5 \mathrm{~mm} / \mathrm{min}$, once the waves develop upstream, they are able to propagate downstream throughout the channel almost immediately. We systematically measured the decay length of the waves as we increased the imposed flow velocity and found that it grows with the advective flow. Figure 2a-d summarizes properties of these boundary-driven waves in terms of their wave velocity, decay length, period, and wavelength. Below, we consider different flow regimes and discuss wave properties in detail.

\section{A. Waves at low flow velocities}

The development of boundary-driven waves at the low flow velocity of $V_{f}=0.3$ is shown in Fig. 3 (see supplementary movie 


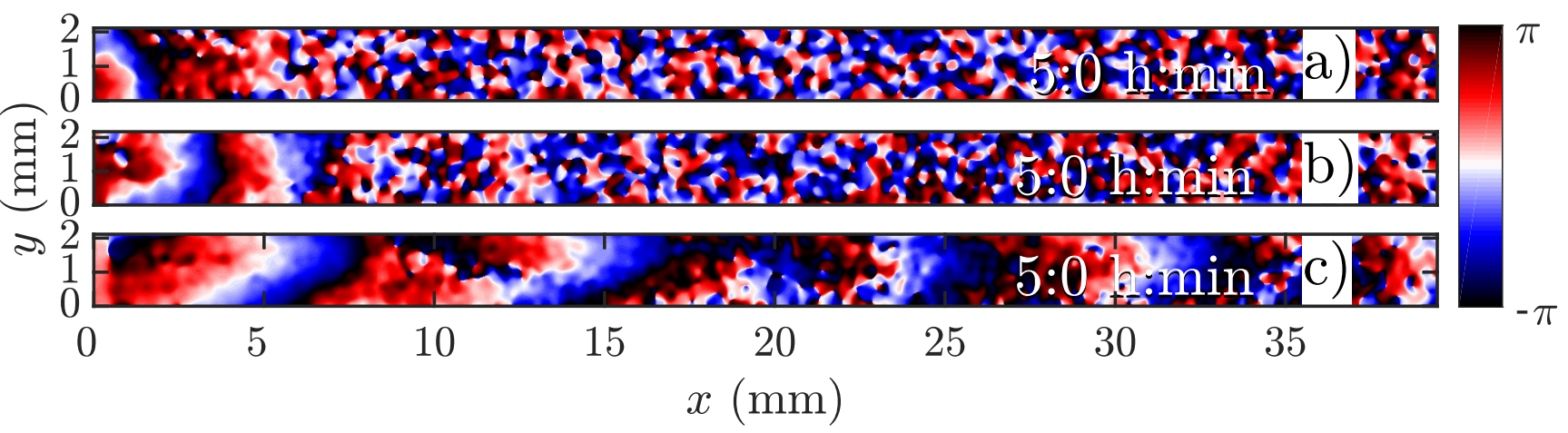

Fig. 1 Phase map of boundary-driven waves in a population of PdsA- cells at a) low flow speed of $V_{f}=0.3 \mathrm{~mm} / \mathrm{min}$, b) moderate flow speed of $V_{f}=0.7 \mathrm{~mm} / \mathrm{min}$ and c) high flow speed of $V_{f}=1.5 \mathrm{~mm} / \mathrm{min}$. While the waves in a) and b) are almost planar and decay along the channel, the waves in c) are parabolic and fill the whole length of the channel (see supplementary movies S1-S3). Timestamps show the time since the flow was turned on.
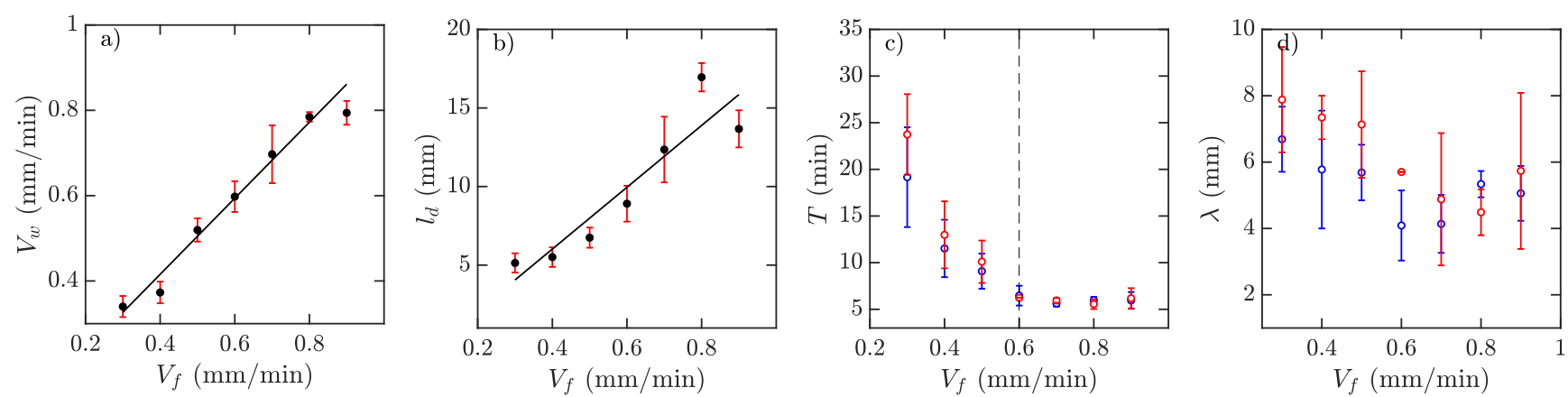

Fig. 2 Experimental characterization of boundary-driven waves relayed by PdsA- cells when subjected to a flow of cAMP-free buffer. a) Wave velocity $\left.V_{w}, \mathrm{~b}\right)$ wave decay length $l_{d}, \mathrm{c}$ ) the period $T$, and d) the wavelength $\lambda$ as a function of the imposed flow velocity $V_{f}$. Solid black lines in a-b) represent a linear least-squares fit to the data. Blue and red data points in c-d) are from the early and late regime of the experiments.

S1). A wave front initiates at the upstream boundary of the channel and decays quickly over a length scale of $\sim 5 \mathrm{~mm}$. Over time, the waves successfully travel a longer distance $(\sim 10 \mathrm{~mm})$ before dying out. We define a characteristic length $l_{d}$ which corresponds to the length that a wave travels inside the channel before its amplitude is lower than $10 \%$ of its maximum amplitude. This length scale grows with the applied flow speed, meaning that the waves reach further downstream in the channel at higher flow speeds. Based on the decay length of the waves at a given flow velocity, we define an early and a late regime. During the early regime, the waves decay after a shorter length than during the later regime. Therefore, we systematically measure the wave period and the wavelength corresponding to each regime separately, as shown in Fig. 2c-d.

An interesting feature of the waves developed in the flow range of $0.3 \leq V_{f} \leq 0.6$ is their large period. We observed periods as large as $20-25 \mathrm{mins}$ for $V_{f}=0.3 \mathrm{~mm} / \mathrm{min}$, decreasing to $10-15$ $\min$ at $V_{f}=0.4 \mathrm{~mm} / \mathrm{min}$ and approaching the normal period of 6 $\min$ at $V_{f}=0.6 \mathrm{~mm} / \mathrm{min}$ (see Fig. 2c). It is plausible that at flow velocities larger than $0.6 \mathrm{~mm} / \mathrm{min}$, the period of the waves is set by the random firing of the cells and not anymore by BDOs. This hypothesis was confirmed by our numerical simulations. Finally, the aggregation pattern of the cells in the presence of boundarydriven waves for $V_{f}=0.3 \mathrm{~mm} / \mathrm{min}$ is shown Fig. 3e. It seems that cells even at the upstream part of the channel that were exposed to boundary-driven waves fail to aggregate. We believe that this is due to the fact that the amplitude of the boundary-driven waves at low flow speeds is not strong enough to trigger aggregation of the cells. The minimum flow speed needed in our experiments to rescue aggregation of the cells was $0.6 \mathrm{~mm} / \mathrm{min}$. Note that this velocity coincides with the velocity at which period of boundarydriven waves sets to the natural period of $6 \mathrm{~min}$ (see the dashed line in Fig. 2c).

\section{B. Waves at moderate flow velocities}

As we increase the flow velocity, in the range $0.6 \mathrm{~mm} / \mathrm{min} \leq V_{f} \leq$ $1 \mathrm{~mm} / \mathrm{min}$, waves behave differently. Although in the early phase, they penetrate only in part of the channel (Fig. 4a,c), in the late phase they successfully propagate throughout the channel (Fig. 4b,d). Moreover, the waves are stronger in amplitude and have the normal period of $6 \mathrm{~min}$, similar to the period of the flow-driven waves in WT cells ${ }^{15,22}$. Regarding the aggregation pattern, we observe clusters of cells mostly in the upper part of the channel, confirming that aggregation is partially rescued. We also performed bright-field measurements to look closely at aggregation of PdsA cells in the presence of flow $\left(V_{f}=1 \mathrm{~mm} / \mathrm{min}\right)$. Figure 5 shows that rescue of the cells is more successful at the upstream end of the channel where the boundary-driven waves penetrate initially and have a larger amplitude (see supplemen- 

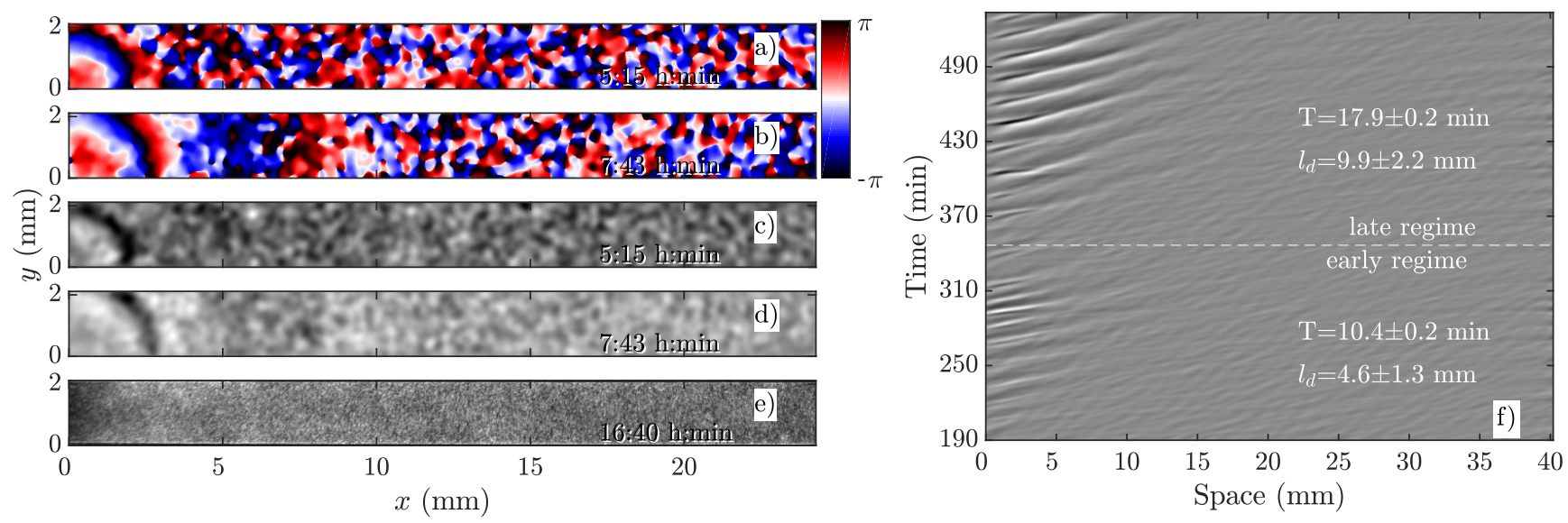

Fig. 3 Overview of an experiment at a low flow speed of $V_{f}=0.3 \mathrm{~mm} / \mathrm{min}$ (see movie $\mathrm{S} 1$ ). a-b) phase images extracted from processed images in c-d). a) and c) show waves during the early regime that decay immediately upon entering the channel. b) and d) show waves during the late regime, which move slightly farther along the channel before decaying. e) shows that the cells do not aggregate even at the end of the experiment. $f$ ) shows part of the space-time plot of this experiment. Timestamps denote time since start of experiment.
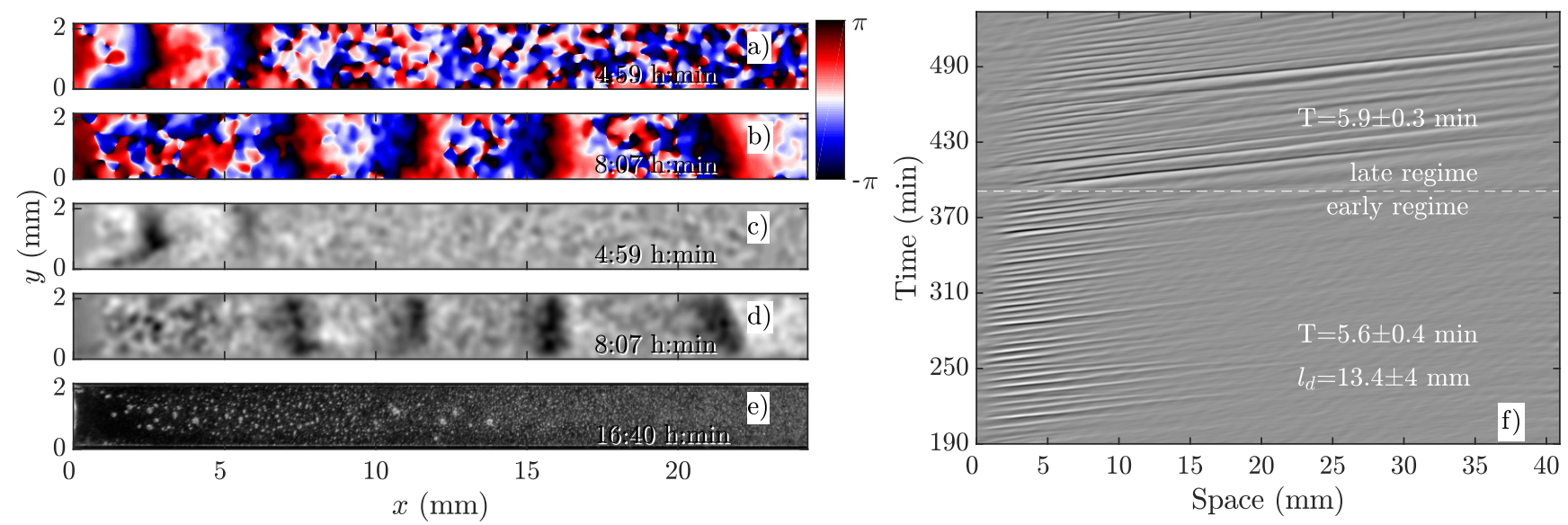

Fig. 4 Overview of an experiment at a medium flow speed of $V_{f}=0.7 \mathrm{~mm} / \mathrm{min}$ (see movie S2). a-b) phase images extracted from processed images in c-d). a) and c) show waves during the early regime that decay quickly along the channel. b) and d) show waves during the late regime, which fill the entire channel. e) shows aggregation at the end of the experiment. f) shows a part of the space-time plot of this experiment. Timestamp denotes time since start of experiment.

tary movie S4). Further downstream, the amplitude of the waves decay and is not strong enough to rescue the cells. At higher flow speed, aggregation clusters cover a larger portion of the channel. We emphasize that cells do not show aggregation clusters if the flow is stopped before they start producing boundary-driven waves.

To examine the effect of different imposed flow velocities in a single experiment, we constructed a channel with a sidearm as in Fig. 6. Since the length of the sidearm is about three times the length of the direct channel, the flow in this channel is about 2.2 times slower. This means that a direct comparison of the behavior at different flow speeds is possible in one experiment. As expected, the waves in the direct channel penetrate further down and are stronger than the ones in the sidearm, as shown in Fig. 6a and supplementary movie S5. Similar to flow-driven waves in WT cells $^{22}$, the width of the wave fronts is larger in the straight part of the channel in comparison to the waves in the sidearm. Finally, the spatial extent of the waves affects the aggregation in the two channel parts. While the cells in the main channel aggregate quite well, there is a little aggregation in the sidearm where the spatial extension of the waves is shorter (see Figure 6b). Note that further downstream the aggregation process appears weaker compared to upstream, which we believe is related to the wave amplitude that decays as it propagates along the channel.

\section{Waves at high flow velocities}

If the imposed flow velocity is increased even further, $V_{f} \geq 1$ $\mathrm{mm} / \mathrm{min}$, the so-called early phase disappears. This means that once the boundary-driven waves initiate at the upstream part, they propagate through the entire length of the channel. Figure 7 shows an experiment at $V_{f}=1.5 \mathrm{~mm} / \mathrm{min}$. The waves have the normal period of $6 \mathrm{~min}$ and wave fronts are more elongated in comparison to small flow experiments, which is similar to flowdriven waves at high speeds described previously for WT cells ${ }^{22}$. Cells over the entire length of the channel are rescued and suc- 


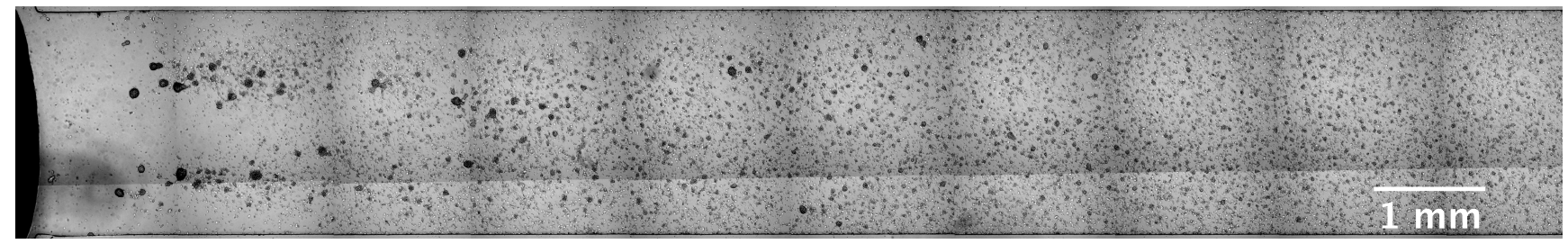

Fig. $5 \mathrm{~A}$ bright-field image showing aggregation of cells in a flow-through channel (see movie S4). Images are taken at $4 \times$ magnification at different parts of the channel and stitched together. Note that at the upstream part of the channel we have a reservoir filled with buffer which is free of the cells and cAMP. Channel width is $2 \mathrm{~mm}$.
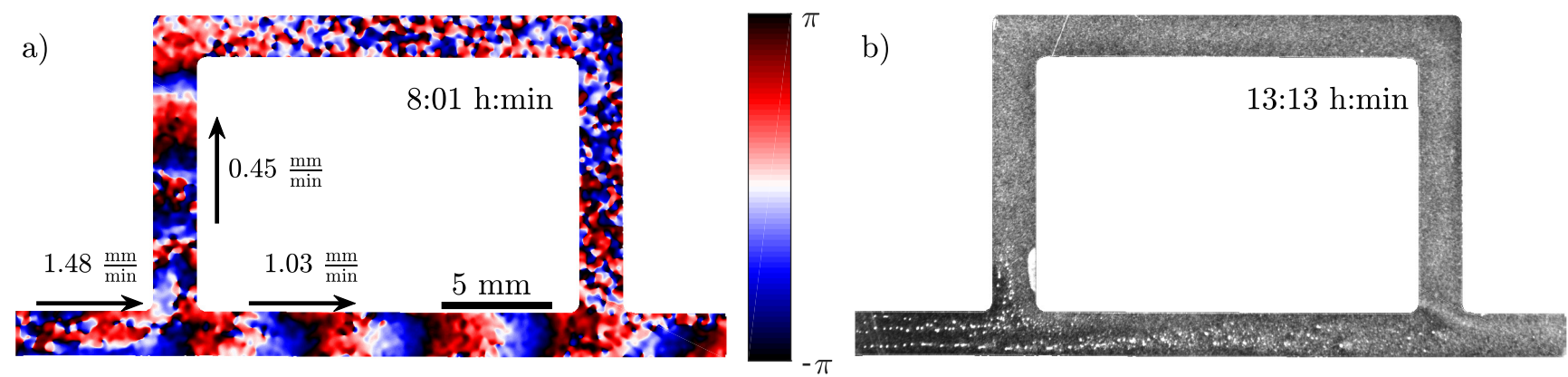

Fig. 6 Experiment in a channel with a sidearm which has a lower flow speed than the straight part of the channel (see movie S5). a) Phase map of the developed boundary-driven waves and b) the corresponding aggregation pattern at the later times. Note different spatial extension of the waves in both parts of the channel which is the important factor in deciding if the aggregation phenotype will be rescued or not.

cessfully aggregate (see Fig. 7e and supplementary movie S3)

\section{Rescue of PdsA` cells by flow}

Another interesting observation in our flow experiments with $\mathrm{PdsA}^{-}$cells was the rescue of pattern formation once the flow was switched off. We exposed the cells to a minimum flow velocity of $V_{f}=0.6 \mathrm{~mm} / \mathrm{min}$ and let the boundary-driven waves develop and propagate for a minimum time of one hour before we switch off the flow. Interestingly, we observed that in less than 90 mins wave centers developed spontaneously and relayed through the population. This occurred again at the upstream parts of the channel that were exposed to boundary-driven waves (see Fig. 8 and supplementary movie S6). The period of the spontaneous waves was significantly higher than the ones produced by wild type cells (1624 min compared to 4-6 min in wild type cells). The wave velocity was measured to be $0.263 \pm 0.006 \mathrm{~mm} / \mathrm{min}$, which is comparable to the wave speed of $0.35 \mathrm{~mm} / \mathrm{min}$ in wild type cells. A systematic measurement of wavelength, period and propagation velocity of the spontaneous waves is presented in Fig. 8b-c. In a single experiment, we measure a wide range of wave periods varying from $15 \mathrm{~min}$ (as they appear) and increasing to $25 \mathrm{~min}$ at later times. This is shown in Fig. 8b.

Based on these results, we explored the minimum time PdsA ${ }^{-}$ cells need to be subjected to flow, such that they show spontaneous pattern formation once the flow is switched off. We found that the cells show spontaneous pattern formation, even if the population experienced the passage of very few boundary-driven waves before the flow was switched off. Figure 9a shows an example where the flow of $V_{f}=2 \mathrm{~mm} / \mathrm{min}$ was turned off once 1-2 pulses of boundary-driven waves pass through the channel. The
cAMP waves form spontaneously at $t \sim 360$ min only at the upstream part of the channel. Interestingly, at flow speed of $V_{f}=5$ $\mathrm{mm} / \mathrm{min}$ where we allow for boundary-driven waves to persist for about 2 hours, once we switch off the flow, the spontaneous waves appear almost immediately again at $t \sim 360 \mathrm{mins}$. Since the flow-driven waves penetrate throughout the channel, the recovered spontaneous waves are also observed over the entire length of the channel (see movies S7,S8).

\section{Rescue of PdsA cells with Wild Type cells}

As we mentioned above, pattern formation in $\mathrm{PdsA}^{-}$cells is impaired because of the lack of cAMP degradation. Therefore, we tried rescuing the cells by using the PdsA produced by Ax2 cells. To do this, we injected PdsA- cells into a channel and filled the reservoir of the channel with Ax2 cells. This reservoir is located upstream from the channel, and is where the fluid accumulates before flowing through the channel. Thus, the cell types were not mixed, but the buffer that flowed through the channel was preconditioned by its exposure to the Ax 2 cells. We find that the behavior of this system depends strongly on the initial starvation time of the Ax2 cells used. In the case of using Ax2 cells without previous starvation, there is again no pattern formation for the first three hours of flow. Nevertheless, when waves emerge they do not decay along the channel, but instead fill the channel immediately for all flow speeds studied. However, if we use Ax2 cells that were initially starved for 4 hours, we find that pattern formation is observed immediately after the flow is switched on. Initially, the waves decay rather quickly, but the decay length increases exponentially over time until the whole channel is filled, see Fig. 10a and supplementary movie S9. The rate at which the 

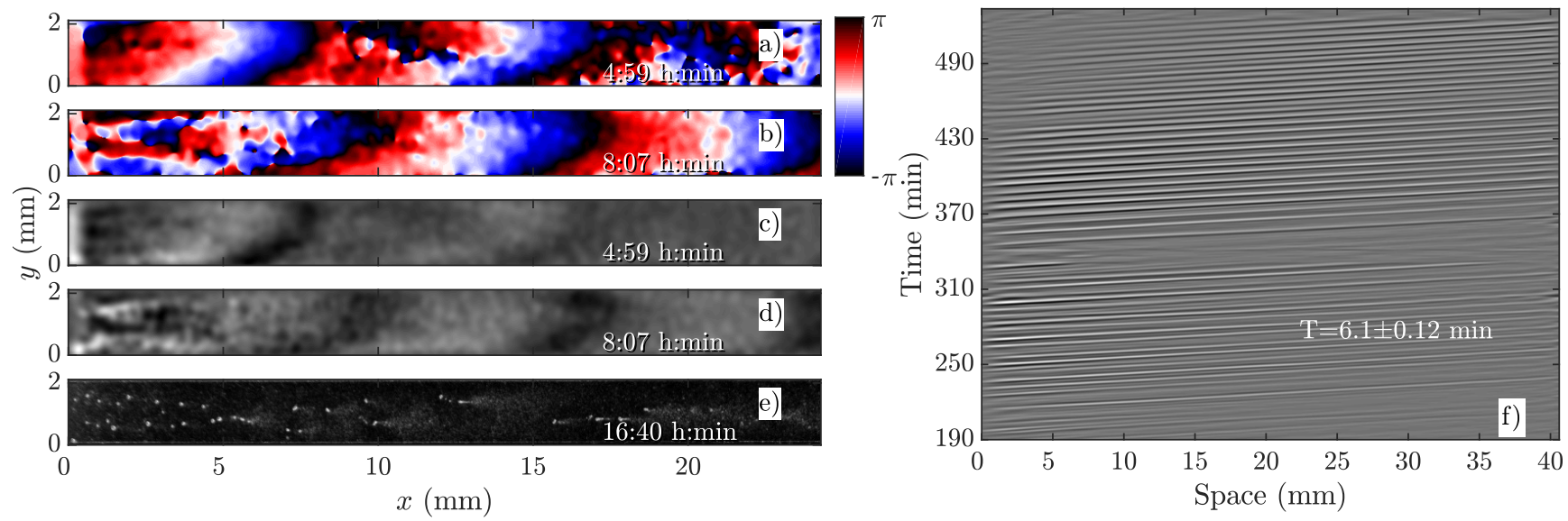

Fig. 7 Boundary-driven waves at $V_{f}=1.5 \mathrm{~mm} / \mathrm{min}$ (see movie S3). a-b) Phase maps extracted from processed images in c-d). e) Cells over the entire length of the channel are rescued and aggregate normally. f) Space-time plot of the waves showing that once the waves develop after three hours of flow, they travel through the entire channel. Timestamps denote time since flow was applied.
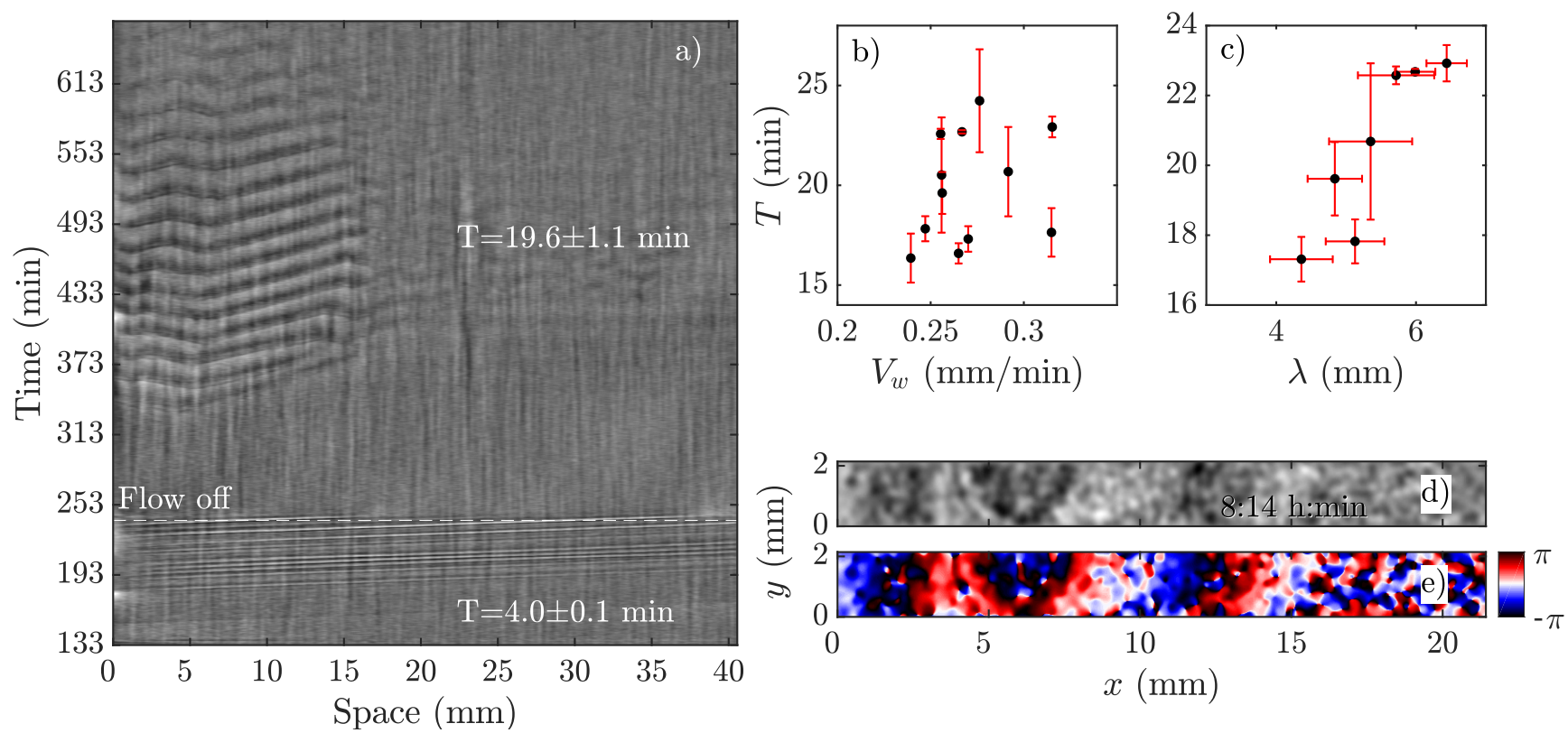

Fig. 8 Pattern formation of PdsA- cells after the flow was switched off. a) Space-time plot of an experiment at imposed flow of $V_{f}=2 \mathrm{~mm} / \mathrm{min}$. The flow was turned off after $240 \mathrm{~min}$ (see movie S6). b) Period $T$ and c) wavelength $\lambda$ of spontaneous waves (appeared in the flow-off regime) plotted as a function of wave velocity $V_{w}$. For these measurements set of different experiments at various flow velocities are used where the flow was switched off at different time points. d) Processed dark-field image and e) the corresponding phase map showing the initiation of a wave at the upstream part of a channel after the flow of $V_{f}=2 \mathrm{~mm} / \mathrm{min}$ has been switched off. The corresponding space-time plot of this experiment is shown in part a). Timestamps show the time since the flow was switched on.

decay length increases depends on the imposed flow velocity, filling the whole channel more quickly for higher advecting flows (see Fig. 10b).

\section{Numerical Simulations of PdsA- Cells}

We performed numerical simulations of the three-component MG model (Eq. 1) at different flow speeds. We selected $\sigma$ and $k_{e}$ as control parameters since they account for the production and degradation of extracellular cAMP, respectively. Depending on these two parameters, this system can have Stable, Bistable and Convectively Unstable (CU) regimes, as is shown in the phase dia- gram in Fig. 11a. We fixed parameters $\sigma$ and $k_{e}$ to be in the stable regime of the phase diagram where the boundary-driven oscillations exist. Note that BDOs exit in the CU regime too, where we have shown ${ }^{1}$ that holding the upstream boundary to a zero concentration of cAMP produces an instability that sends periodic wave trains downstream. The wave generation mechanism works by first advecting the cAMP downstream, thus depleting the upstream area of cAMP. This low concentration of the chemoattractant destabilizes the cells close to the upstream boundary, which react by releasing a pulse of cAMP. For a careful analysis of BDOs in the $\mathrm{CU}$ regime, we refer the reader to Ref. ${ }^{1}$. 

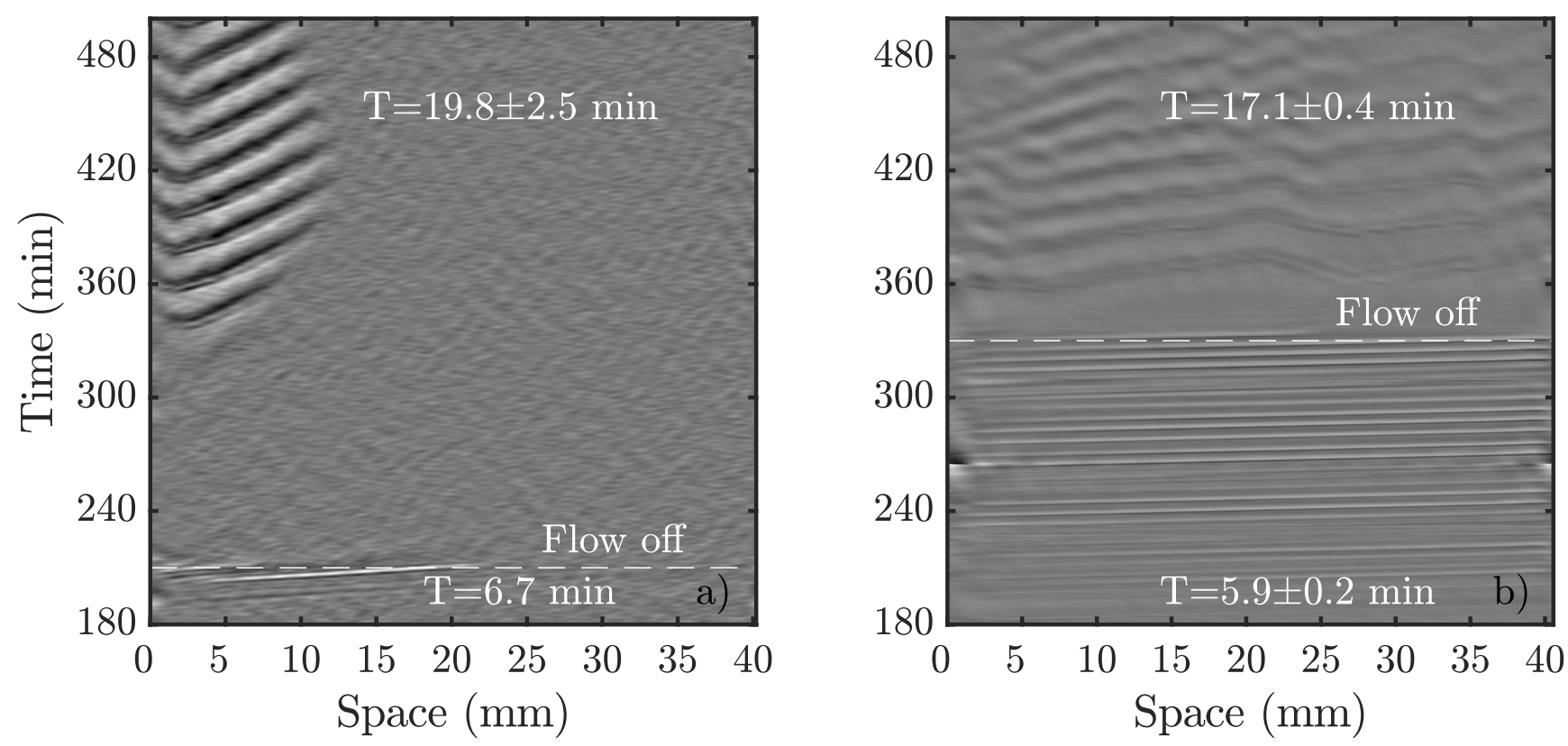

Fig. 9 Comparison of spontaneous pattern formation once the flow is switched off. a) Flow of $V_{f}=2 \mathrm{~mm} / \mathrm{min}$ was switched off shortly after boundary-driven waves were observed. cAMP waves spontaneously appear at $360 \mathrm{~min}$ (see movie S7). b) Higher flow velocity of $V_{f}=5 \mathrm{~mm} / \mathrm{min}$ triggered boundary-driven waves that propagated through the population for more than two hours before the flow was turned off. The spontaneous waves appeared almost immediately (see movie S8).
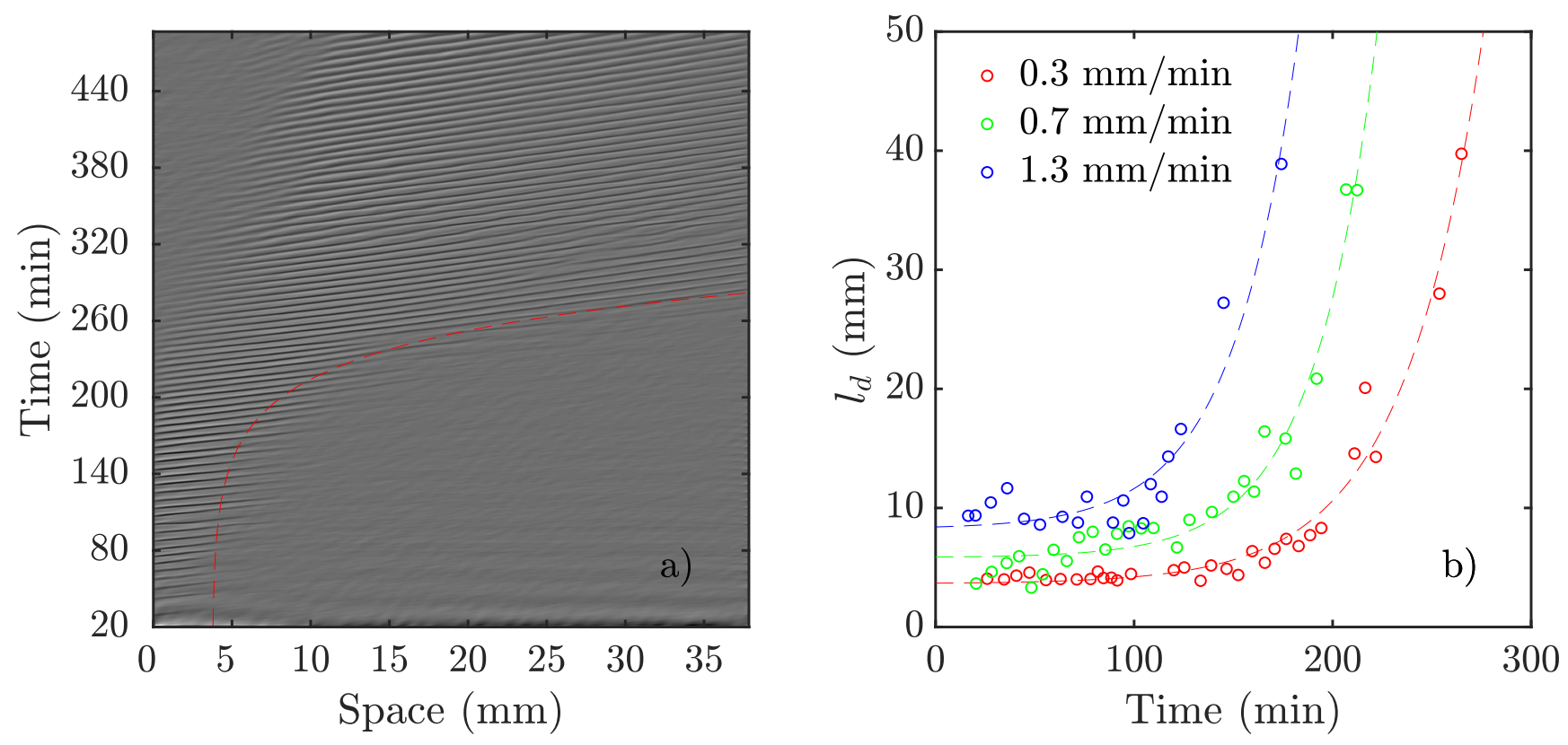

Fig. 10 Flow-driven waves in experiments consisting of a channel filled with $\mathrm{PdsA}^{-}$cells while the reservoir was filled with Ax2 cells starved initially under agitation for 4 hours (see movie S9). a) An exemplary space-time plot at $V_{f}=0.7 \mathrm{~mm} / \mathrm{min}$ with a wave period of $6.2 \pm 0.3 \mathrm{~min}$. The red dashed line illustrates the change of decay length over time (see movie S9). In b) the decay length is plotted vs experiment time for different flow speeds. Dashed lines show least-squares fit of exponential functions to the data of the same color. For each data set a function of the form of $f(x)=a \cdot \exp (b \cdot x)+c$ was used. The fitting values are: $a_{0.3}=0.0474 \mathrm{~mm}, b_{0.3}=0.0250 \mathrm{~min}^{-1}, c_{0.3}=3.6374 \mathrm{~mm}, a_{0.7}=0.0396 \mathrm{~mm}, b_{0.7}=0.0316 \mathrm{~min}-1$, $c_{0.7}=5.8376 \mathrm{~mm}$ and $a_{1.3}=0.1636 \mathrm{~mm}, b_{1.3}=0.0303 \mathrm{~min}^{-1}, c_{1.3}=8.2406 \mathrm{~mm}$.

To mimic our experimental setup, we performed numerical simulations in a channel filled with PdsA- cells with imposition of a Dirichlet boundary upstream. To account for the low degradation rate of the cells, we consider small values of degradation rate $k_{e}$. Numerical simulations showed that at low-speed flows the system reaches a time independent steady state. This solution is a monotonic curve that increases from a zero cAMP concentration at the channel upstream boundary up to a very high concentration in the channel downstream end (see Fig. 11b). At higher velocities of the advecting flow an instability appears, and the system's 

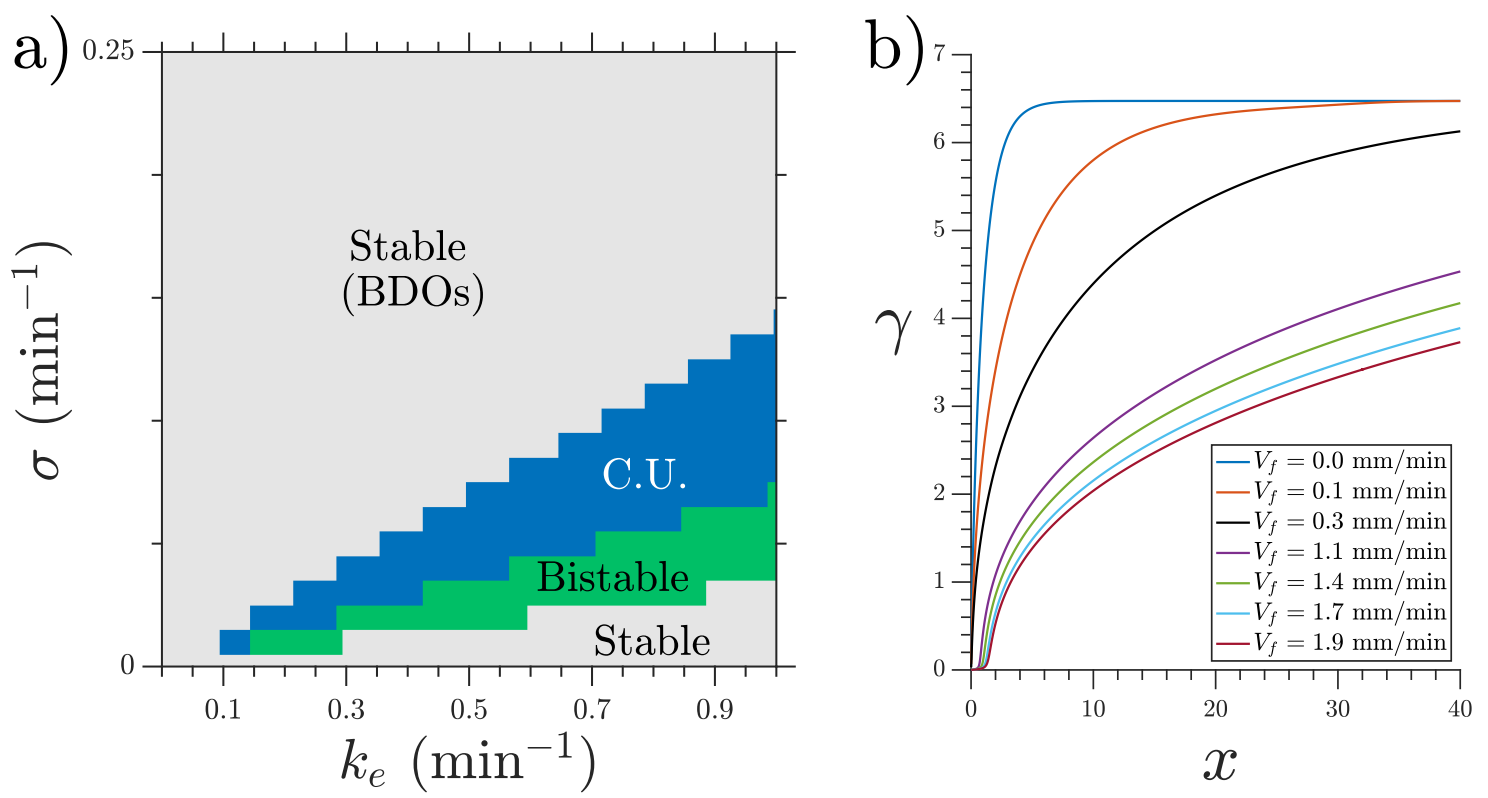

Fig. 11 a) Phase diagram of the MG model showing the range at which BDOs exist. Note that BDOs exist in both stable and connectively unstable $(\mathrm{CU})$ regimes. b) Steady state cAMP concentration $\gamma$ obtained at $\sigma=0.3 \mathrm{~min}^{-1}$ and $k_{e}=0.01 \mathrm{~min}^{-1}$. BDOs appear in the velocity range of 0.4 $\mathrm{mm} / \mathrm{min} \leq V_{f} \leq 0.8 \mathrm{~mm} / \mathrm{min}$.

solution is no longer time independent. The upstream boundary periodically emits waves that decay as they travel along the channel, similar to those observed in experiments. Examples of boundary-driven waves at three different flow speeds are shown in Fig. 12a-c and supplementary movies S10-S12. We emphasize that this instability only appears when an absorbing boundary condition in the upstream boundary is enforced, thus emulating the advection of cAMP-free buffer.

We studied the properties of these waves in a 1D geometry along a range of cAMP production rate intensities $(\sigma)$ and velocities, while keeping the degradation of cAMP very low $\left(k_{e}=0.01\right.$ $\left.\min ^{-1}\right)$. The properties of these waves are summarized in Figure 13; it can be seen that the period of the advected waves increases with the advecting flows, while the characteristic length $l_{d}$ increases with flow velocity. The existence of these waves is restricted to a range of velocities, which increases to include faster flows at higher production rates of cAMP. Once the advecting flows are too fast for this mechanism to exist another numerical scheme needs to be used to periodically inject waves into the system. The high speed behavior is consistent with the previously reported behavior of $\mathrm{WT}^{22}$ where the period is independent of the advecting flows with $T \approx 5-6 \mathrm{~min}$.

We also performed numerical simulations emulating the experiments with WT cells in the inlet. In those simulations the parameters were fixed as $k_{e}=5.0 \mathrm{~min}^{-1}$ and $\sigma=0.55 \mathrm{~min}^{-1}$ for $x<l$ with $l=1 \mathrm{~mm}$ to represent the inlet. Under these parameters, the inlet oscillates with a fixed period and emits waves to the rest of the channel. In simulations including phosphodiesterase advection and WT cells in the inlet we observed that the secreted phosphodiesterase was advected quickly downstream and therefore the phosphodiesterase concentration could be assumed constant along the channel during most of the experiment. Given this phosphodiesterase distribution, in the experiments when the cells are not prestarved and signaling starts after 3 hours the system behaves like a channel filled with WT.

In the case where the WT cells are prestarved and start to fire immediately after being placed in the channel we found that an increasing production rate of cAMP for the PdsA cells reproduced the increasing decaying length in the system. Simulations for different production rates with fixed degradation rates (fixed at WT values $k_{e}=5.0 \mathrm{~min}$ ) were performed, and their decay length increased with increasing production rate, as is shown in Figure 14a. An example of a simulation where the production rate increased over time as $\sigma=K t$ ( $K$ is a constant) can be seen in Fig. 14b; there, the decay length increases over time (see movie S11).

\section{Discussion}

Previous studies have shown that pulsing PdsA' cells with cAMP is not enough to rescue them ${ }^{13,27}$. Due to the lack of degradation in a population of PdsA'cells, cAMP accumulates without producing waves, preventing the cells from aggregating naturally. It has been shown that adding external phosphodiesterase from a rat brain ${ }^{27}$, PDE overexpressors ${ }^{13}$ or by mixing with WT cells ${ }^{28}$ causes $\mathrm{PdsA}^{-}$cells recover their aggregation capabilities.

Here, we have shown that an influx of cAMP-free buffer does not only recover signaling in PdsA deficient cells, but also that these cells are capable of signaling and aggregation once the flow has been switched off. In our flow-through microfluidic setup filled with PdsA ${ }^{-}$cells we observed cAMP waves after 3 hours of starvation with flow, independent of whether or not they were previously starved in a shaker. This confirms that reduced levels of cAMP are necessary for their development. These waves have a finite decay length inside the channel which grows with the 

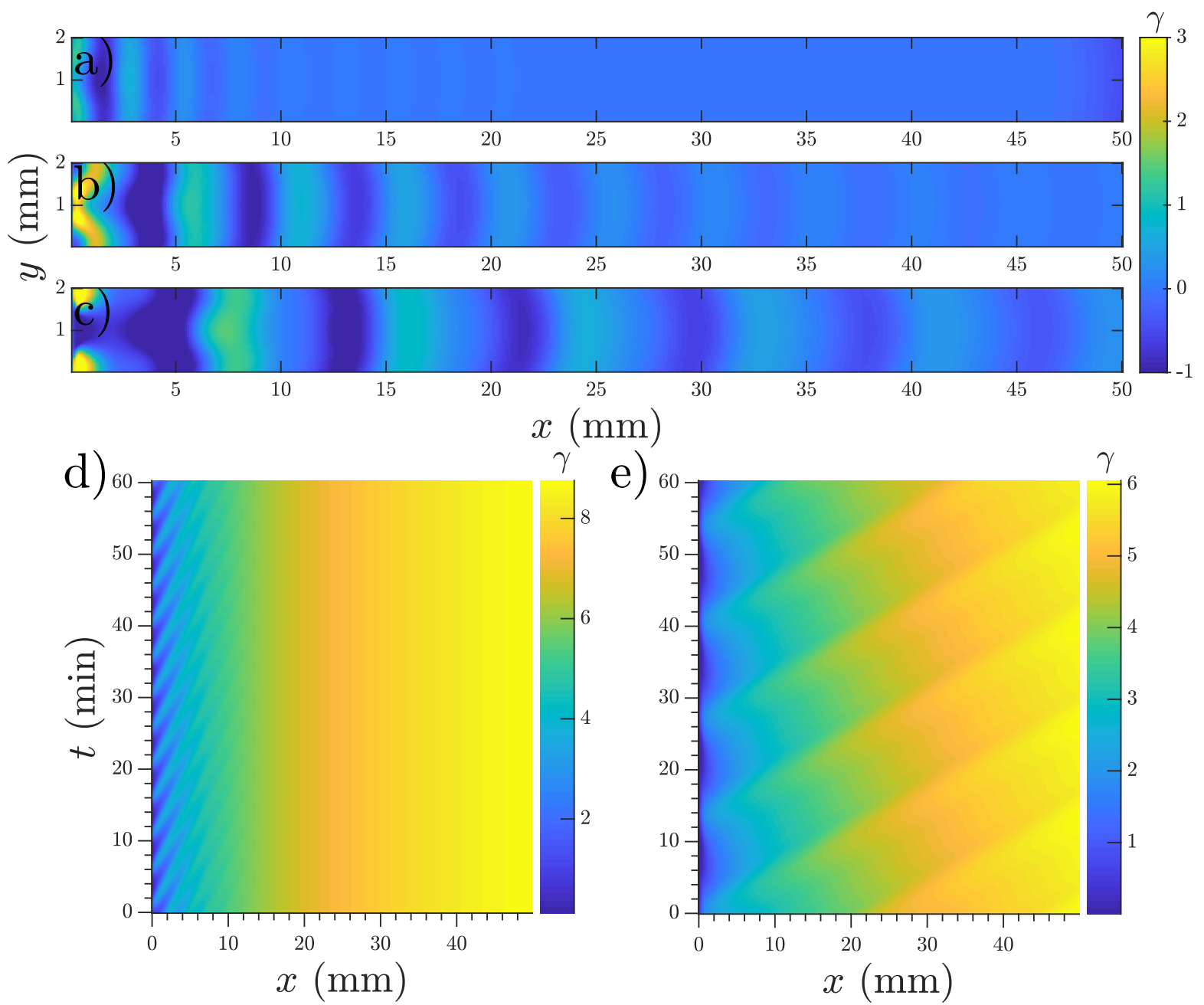

35

45

50

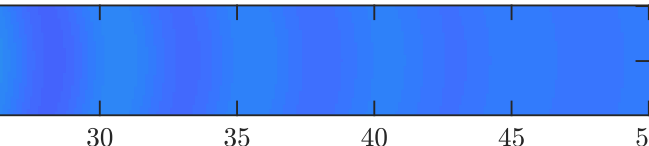

3

40

45

50

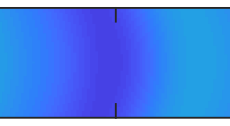

$30 \quad 35$

$35 \quad 40$

$40 \quad 45$

45

50

(e)

$\gamma$ e)

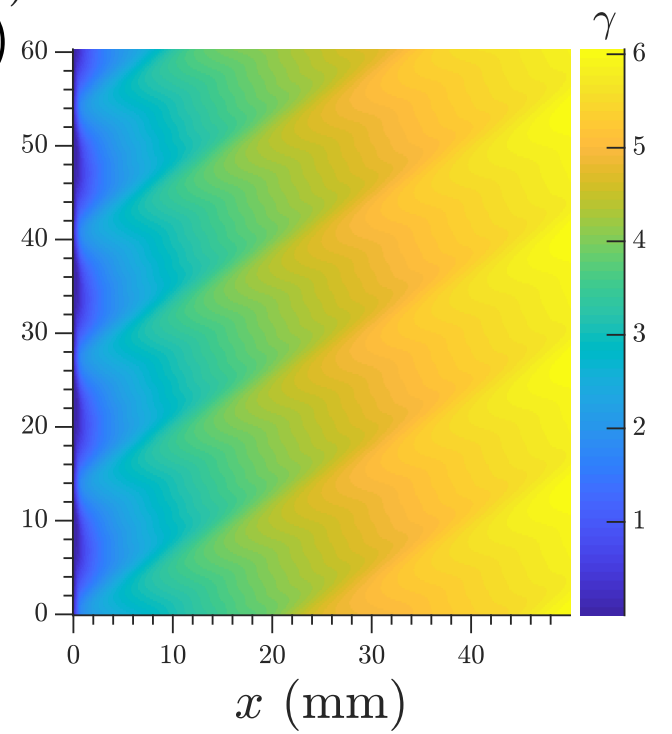

Fig. 12 Boundary-driven waves developed in a channel with Dirichlet boundary condition at $x=0$. a) $\left.V_{f}=0.5 \mathrm{~mm} / \mathrm{min}, \mathrm{b}\right) V_{f}=0.8 \mathrm{~mm} / \mathrm{min}$ and c) $V_{f}=1.1 \mathrm{~mm} / \mathrm{min}$ (see movies S10-S12). Waves penetrate over longer distances at higher flow velocities. d-e) Space-time plot of boundary-driven waves at imposed flow velocities of $0.5 \mathrm{~mm} / \mathrm{min}$ and $2 \mathrm{~mm} / \mathrm{min}$ showing higher periods at higher flow speeds. Other parameters are $\sigma=0.6 \mathrm{~min}-1$ and $k_{e}=0.01 \mathrm{~min}^{-1}$. Note that in a)-c) the mean value of $\gamma$ is subtracted to enhance the contrast of the waves. As a result, $\gamma$ finds negative values and its range is different from space-time plots in parts d)-e).

imposed flow. These boundary-driven waves develop the cells, and the waves reach further down the channel after around 6.5 hours throughout the experiment. Since cells continue signaling after the flow is stopped it is plausible that other types of phosphodiesterases such as PDE4 take over the role of PdsA during aggregation ${ }^{12}$, having a higher activity than in the normal presence of PdsA. Other biochemical experiments are necessary to confirm this hypothesis. Interestingly, the period of the spontaneous waves observed in the recovered population of PdsA' cells was much higher than that of the WT cells, and the rescued cells were located on the upstream of the channel, consistent with the area covered by the decaying boundary-driven waves.

Our numerical simulations show the nature of the instability that produces the waves in this system. A flow of cAMP-free buffer provides the destabilizing mechanism necessary for wave production, thus showing boundary-driven oscillations. At small flow speeds the clean buffer produces depletion of cAMP in the upstream edge of the channel, thus allowing the cells located there to fire and produce a cAMP wave. As this wave travels downstream the basal concentration of cAMP increases and the wave loses amplitude, decaying as it travels. At higher flow speeds more clean buffer penetrates the system, thus the basal cAMP concentration increases more slowly along the channel (smaller gradients), therefore allowing the waves to travel further. In our simulations there is a maximum velocity such that the boundarydriven oscillations exist. This is in disagreement with our experimental observations, where at higher flow speeds wave generation continues to occur. We believe that the range of existence of this instability is marked by the change in the oscillating period observed in experiments (see the vertical line in Fig. 2c). For $V_{f}<0.6 \mathrm{~mm} / \mathrm{min}$ the wave period is large and changes with the advecting flow, while for $V_{f}>0.6 \mathrm{~mm} / \mathrm{min}$ it is constant around $T \approx 5-6 \mathrm{~min}$, therefore these BDOs exist for $V_{f}<0.6 \mathrm{~mm} / \mathrm{min}$. To test this hypothesis we performed numerical simulations including a constant firing of the cells with $T=6 \mathrm{~min}$. For the range of velocities where the boundary-driven oscillations exist, the period 

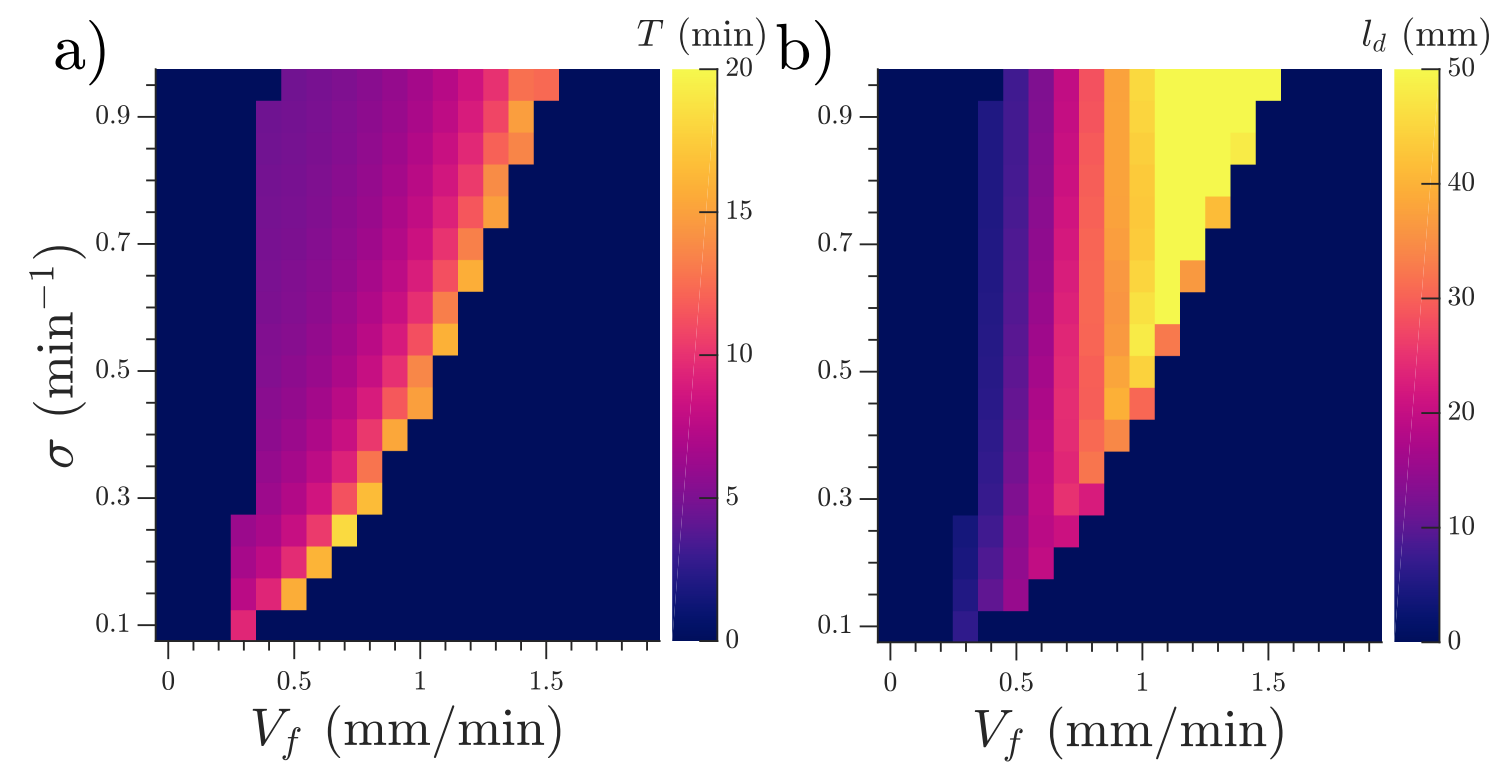

Fig. $13 \mathrm{a}$ ) Period $T$ and b) characteristic decay length $l_{d}$ of the observed waves as functions of the velocity of the advecting flow $V_{f}$ and the production rate $\sigma$ of cAMP. Note that simulations are performed in a $1 \mathrm{D}$ geometry. Degradation rate of $\mathrm{cAMP}$ is $k_{e}=0.01 \mathrm{~min}^{-1}$ and channel length is $L=50$ $\mathrm{mm}$.
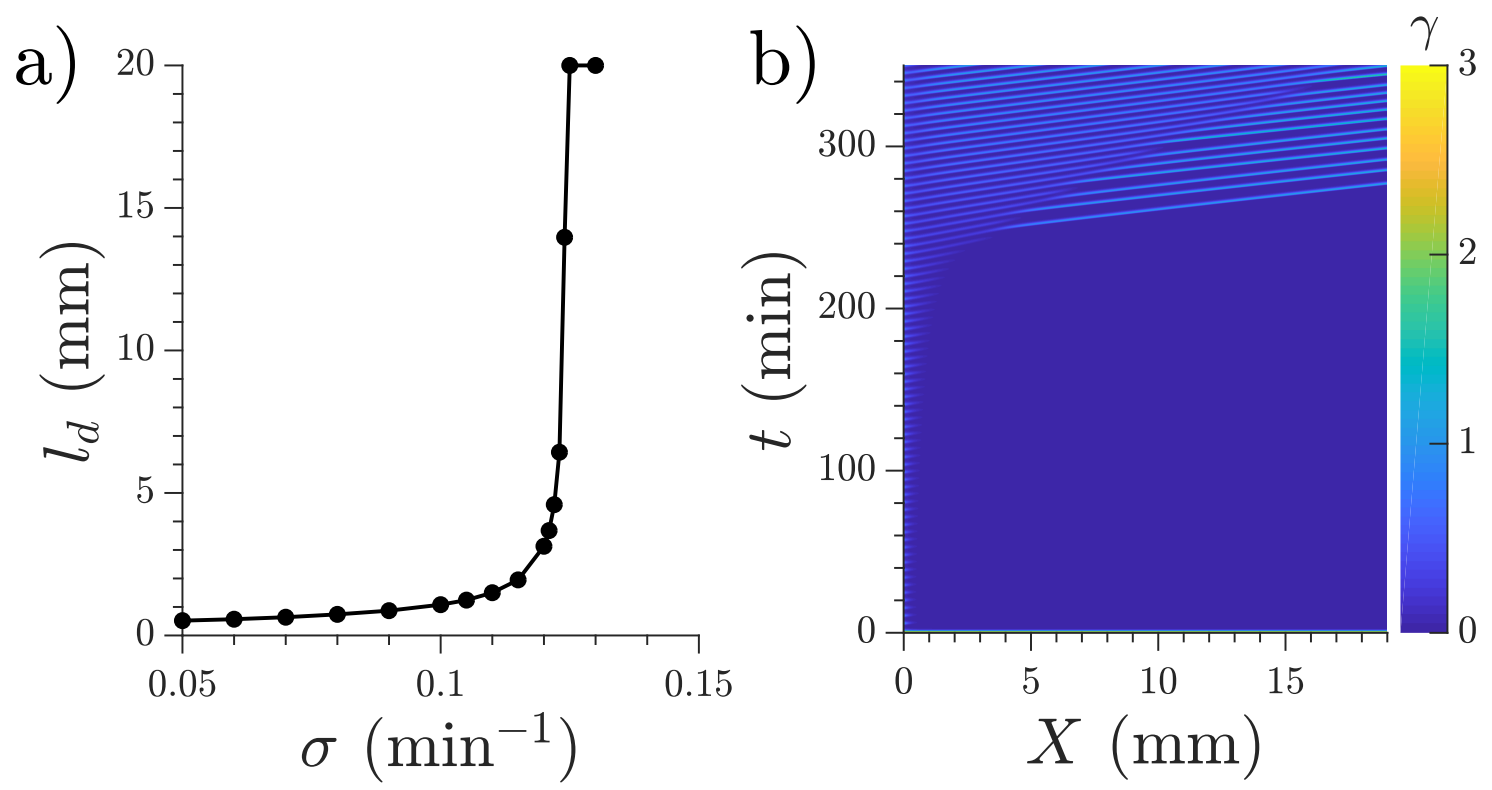

Fig. 14 a) Characteristic length $l_{d}$ for the waves produced at the inlet by the WT cells. The degradation rate given by the effect of phosphodiesterase is fixed at $k_{e}=5.0 \mathrm{~min}^{-1}$ for the whole channel. b) Space-time representation of a numerical simulation with WT cells in the inlet (not shown) and linearly increasing CAMP production rate in the rest of the channel, $\sigma=0.001 \cdot t$. Flow velocity is $0.7 \mathrm{~mm} / \mathrm{min}(\mathrm{see} \mathrm{movie} \mathrm{S} 13$ ).

of these oscillations prevailed and the firing had no effect on the system. For higher velocities the constant firing sets the system's period.

Moreover, we used WT cells in the upstream end of the channel to rescue PdsA- cells. We observed waves with increasing penetration length that propagated inside the channel. This behavior was reproduced in our simulations with WT cells at the reservoir with an increasing cAMP production rate of $\mathrm{PdsA}^{-}$cells everywhere in the channel. Therefore, we attribute this increasing penetration length to the gradual development of the PdsA' cells in the channel. If the WT cells at the reservoir are not prestarved, then the WT cells develop along with the PdsA- cells and the flowdriven waves appear after 3 hours and are capable of penetrating throughout the channel.

Finally, it is shown that in a reaction-diffusion system Dirichlet boundary conditions are able to destabilize the uniform steady state and generate stable, spatially non-uniform patterns ${ }^{29}$, which can be applied to many patterning phenomena in biology, ecology or chemical systems. In this work, we show that by imposition of Dirichlet boundary condition in experiment or numer- 
ical simulations, boundary-driven instabilities are relevant in the presence of advection. This could be a plausible mechanism in order to generate a continuous periodic influx of wave trains in an otherwise stable reaction-diffusion system.

\section{Conflicts of interest}

There are no conflicts to declare.

\section{Author Contributions}

A.G. designed the research. E.V.H. designed and carried out numerical simulations. T.E. performed experiments. E.V.H., T.E. and A. G. analyzed data. A.G., E.V.H. and T.E. wrote the manuscript. E.V.H. and T.E. contributed equally.

\section{Acknowledgments}

The authors thank M. S. Müller and K. Gunkel for their cheerful help with the preparation of the cells. T. E. acknowledges Deutsche Forschungsgemeinschaft (DFG), project number GH 184/1-1. E.V.H. thanks the Deutsche Akademische Austauschdienst (DAAD), Research Grants-Doctoral Programs in Germany. A.G. acknowledges MaxSynBio Consortium, which is jointly funded by the Federal Ministry of Education and Research of Germany and the Max Planck Society.

\section{References}

1 E. Vidal-Henriquez, V. Zykov, E. Bodenschatz and A. Gholami, Chaos: An Interdisciplinary Journal of Nonlinear Science, 2017, 27, 103110.

2 E. Pálsson and E. C. Cox, Proceedings of the National Academy of Sciences, 1996, 93, 1151-1155.

3 J. J. Tyson, K. A. Alexander, V. Manoranjan and J. Murray, Physica D: Nonlinear Phenomena, 1989, 34, 193-207.

4 D. A. Kessler and H. Levine, Phys. Rev. E, 1993, 48, 48014804.

5 S. Almeida and R. Dilão, Physical Review E, 2016, 93, 052402.

6 J. T. Bonner, Cellular slime molds, Princeton University Press, Woodstock, 2015, vol. 2127.

7 W. Loomis, Dictyostelium discoideum: a developmental system, Elsevier, Amsterdam, 2012.

8 A. Theibert and P. N. Devreotes, The Journal of cell biology, 1983, 97, 173-177.

9 C. Klein and M. H. Juliani, Cell, 1977, 10, 329-335.
10 D. Hereld, R. Vaughan, J. Y. Kim, J. Borleis and P. Devreotes, Journal of Biological Chemistry, 1994, 269, 7036-7044.

11 P. N. Devreotes and J. Sherring, Journal of Biological Chemistry, 1985, 260, 6378-6384.

12 S. Bader, A. Kortholt and P. J. Van Haastert, Biochemical Journal, 2007, 402, 153-161.

13 G. L. Garcia, E. C. Rericha, C. D. Heger, P. K. Goldsmith and C. A. Parent, Molecular biology of the cell, 2009, 20, 32953304.

14 T. Gregor, K. Fujimoto, N. Masaki and S. Sawai, Science, 2010, 328, 1021-1025.

15 A. Gholami, O. Steinbock, V. Zykov and E. Bodenschatz, Physical review letters, 2015, 114, 018103.

16 A. Gholami, O. Steinbock, V. Zykov and E. Bodenschatz, New Journal of Physics, 2015, 17, 063007.

17 G. M. Whitesides, E. Ostuni, S. Takayama, X. Jiang and D. E. Ingber, Annual review of biomedical engineering, 2001, 3, 335373.

18 A. D. Edelstein, M. A. Tsuchida, N. Amodaj, H. Pinkard, R. D. Vale and N. Stuurman, Journal of biological methods, 2014, 1, e10.

19 D. A. Egolf, I. V. Melnikov and E. Bodenschatz, Physical Review Letters, 1998, 80, 3228.

20 J.-L. Martiel and A. Goldbeter, Biophysical journal, 1987, 52, 807.

21 J. Lauzeral, J. Halloy and A. Goldbeter, Proceedings of the National Academy of Sciences, 1997, 94, 9153-9158.

22 T. Eckstein, E. Vidal-Henriquez, A. Bae, V. Zykov, E. Bodenschatz and A. Gholami, PLOS ONE, 2018, 13, 1-20.

23 E. Vidal-Henriquez and A. Gholami, Scientific reports, 2019 , 9, 3935.

24 E. Pálsson, Biophysical journal, 2009, 97, 2388-2398.

25 R. Merson, Proc. Symp. Data Processing, 1957, pp. 1-25.

26 B. Koren, Numerical methods for advection-diffusion problems, Vieweg, Braunschweig, 1993, pp. 117-138.

27 M. Darmon, J. Barra and P. Brachet, Journal of cell science, 1978, 31, 233-243.

28 R. Sucgang, C. J. Weijer, F. Siegert, J. Franke and R. H. Kessin, Developmental biology, 1997, 192, 181-192.

29 P. Maini and M. Myerscough, Applied Mathematics Letters, 1997, 10, 1-4. 



\section{Spatial heterogeneities shape collective behavior of signaling amoeboid cells}

The following paper draft is the base for a manuscript that has been accepted by Science Signaling as Eckstein T, Vidal-Henriquez E, Bae A and Gholami A. Spatial heterogeneities shape collective behavior of signaling amoeboid cells

TE performed experiments. All authors analyzed data. All authors wrote the initial manuscript. All authors reviewed and edited the manuscript. TE produced figures 1, 2, 3, 4, S1, S2, S3, S4, S5, S6, S7, S9, S10, S15C) and S16. 


\title{
Spatial heterogeneities shape collective behavior of signaling amoeboid cells
}

\section{Control of pattern formation in signaling amoeboids}

\author{
T. Eckstein ${ }^{1 \dagger}$, E. Vidal-Henriquez ${ }^{1 \dagger}$, A. J. Bae ${ }^{1 \ddagger}$, A. Gholami ${ }^{1 *}$ \\ ${ }^{1}$ Max Planck Institute for Dynamics and Self-Organization, Am Fassberg 17, \\ 37077 Göttingen, Germany \\ *For correspondence: azam.gholami@ds.mpg.de \\ $\dagger$ These authors contributed equally to this work \\ ${ }^{\ddagger}$ Present address: Department of Biomedical Engineering, University of Rochester, USA
}

\begin{abstract}
In their natural habitat in the forest soil, the cellular slime mold Dictyostelium discoideum is exposed to spatial heterogeneities. To date, the influence of these environmental inhomogeneities on signaling and pattern formation of Dictyostelium cells is not well understood. We present novel experimental results as well as comprehensive numerical simulations on pattern formation of signaling amoeba cells in the presence of periodic arrays of millimeter-sized pillars. We observe concentric cAMP waves that initiate almost synchronously at the pillars and propagate outwards. The cells respond chemotactically to these circular waves and stream towards the pillars, forming periodic domains that reflect the periodicity of the underlying lattice. Our results suggest that in nature the excitability threshold and synchronization level of the cells are two key parameters that control the nature of interaction between cells and the spatial heterogeneities.
\end{abstract}

\section{Introduction}

A fundamental process occurring in excitable reaction-diffusion systems is the propagation of nonlinear waves (1-4). Examples of such waves include chemical waves in the Belousov-Zhabotinsky reaction (5), waves of $\mathrm{CO}$ oxidation on $\mathrm{Pt}$ catalytic surfaces (6), electrical waves in retinal and cortical nerve tissue (7), waves in heart muscle (8), and cAMP (cyclic adenosine monophosphate) waves in starved populations of Dictyostelium discoideum (D.d.) amobae (9, 10). One of the important questions concerning nonlinear waves is how they propagate in the presence of obstacles. Various aspects of this question, such as the influence of one, a few large obstacles, or a large number of small obstacles on wave propagation in excitable media, have been the focus of several theoretical and experimental investigations (11-15).

Target patterns and spiral waves are the basic spatio-temporal patterns in excitable media. It is known that obstacles can stabilize rotating spiral waves by pinning the spiral core $(8,16)$ and understanding the interaction between spirals and obstacles is important in various physical, chemical and biological systems $(8,16-19)$. For example in the heterogeneous heart tissue, once a free rotating spiral pins to an anatomical obstacle, physiologically life-threatening cardiac arrhythmias such as fibrillation can occur $(15,20,21)$. 
Signaling of D.d. cells is a classical example of an excitable medium and shows properties similar to heart tissue $(20,21)$ or chemical waves in BZ reaction (5). This organism, naturally occurring in the forest soil, is an important model system for the study of chemotaxis, cell differentiation, and morphogenesis (22). Starvation of D.d. cells induces a developmental program in which cells align to form head-to-tail streams by signaling to each other with cAMP. Cells initiate the process by sending out pulsatile signals with a periodicity of several minutes, which propagate as waves. Over time, circular and spiral patterns form and cells respond chemotactically to cAMP waves that guide cell movement towards the signaling centers and form multi-cellular centimeter-scale domains. The corresponding wave sources in each domain then act as aggregation centers, which eventually transform into millimeter long slugs and finally into fruiting bodies, bearing spores for long-term survival and long-range dispersal (23). In their natural habitat, populations of starving cells are exposed to spatial heterogeneities, that will profoundly influence the processes of wave generation, propagation, and aggregation. As D.d. cells attempt to detect cAMP gradients and move directionally towards the source, obstacles may block the physical path of the cells or alter the flux of the chemical signal. It is speculated that chemotactic cells can avoid the obstacles by following the altered signal (24), or it is suggested that a self-assisted migration mechanism improves the navigation efficiency of the signaling cells in a complex environment (25). Here, we report experimental and numerical results on the spatio-temporal dynamics of populations of D.d. cells in the presence of non-excitable obstacles. We show that chemotactic sensitivity and synchronization level of the D.d. cells are crucial parameters determining the interaction between cells and the obstacles. Our experiments and numerical simulations confirm that in a synchronized population of starving cells with an increased level of chemotactic sensitivity, inert obstacles can act as aggregation centers if small accumulation of either cells or cAMP occurs around them. However, unsynchronized cells with reduced level of chemotactic sensitivity ignore the obstacles. These results suggest that in nature an optimized level of synchrony and chemotactic sensitivity of the cells control the ability of the cells to signal each other and successfully navigate in the presence of physical constrains. We expect our experiments to help understanding aggregation in complex environments, and more broadly, how self-organizing living systems regulate their parameters in order to survive according to their particular reaction-diffusion characteristics and environmental restrictions.

\section{Results}

In nature, the obstacles encountered by D.d. are randomly distributed in $3 \mathrm{D}$, but as a first step towards understanding, we look at a simpler system of cells in a $2 \mathrm{D}$ geometry with a periodic arrangement of obstacles. Our quasi 2D geometry consists of a regular array of millimeter-sized pillars that control the spatio-temporal dynamics of a population of uniformly distributed D.d. cells (see Fig. S1). Unlike other excitable systems $(13,17)$, initial experiments showed no interaction between obstacles and the appearing cAMP waves. In our experiments, we observed spirals pinned to the unexcitable obstacles when they, by chance, appeared close to one (see Fig S2 and Video 1), but showed no systematic attraction or repulsion to them (see Fig. S3A-B and Video 2). 
Following the results of our numerical simulations (see next section) we lowered the excitation threshold of the cells by adding caffeine to the system. The resulting aggregation pattern is shown in Fig. 1. Under these conditions, the spatial heterogeneities induce circular waves centered on the pillars that trigger chemotactic cell movement towards the pillars. This leads to the formation of periodic domains that reflect the periodicity of the underlying macro-pillar array. Interestingly, we observed synchronized circular waves and regular domains only in the presence of caffeine (see Fig. S3A-B and Video 2 for an experiment in the absence of caffeine). Moreover, the phenomenon showed to be robust to the type of obstacles used, being consistent also for holes and walls.

In the absence of pillars but in the presence of caffeine, target patterns emerge at random locations on the PDMS substrate (Fig. S3C-D and Video 3). This is in contrast with patterns in the presence of macro-pillars (see Video 4), where waves originate at the pillars and propagate outwards, as shown in Fig. 1. The four successive snapshots in Fig. 1A-D reveal circular waves centered around the pillars, cells streaming towards the pillars, formation of regular domains, and cell aggregation, respectively. In our experiments, we observed that concentric waves develop almost synchronously around the pillars and, since they have a slightly higher frequency, dominate over the other firing centers (see supplemental Fig. S4 for a careful analysis of the frequency of firing centers; see also Fig. S5). Waves propagate outwards from the pillars and trigger chemotactic movement of the cells towards the pillars. As a result, periodic domains form around the obstacles. The concentric waves around the pillars are well visible in the spatial phase map in Fig. 1E as well as in the processed Video 5. In this video, the frames in Video 4 are subtracted every $1 \mathrm{~min}$ and band-passed filtered to reduce the spatial noise. To calculate the phase at each pixel, first we applied a filter in frequency domain on Video 5 and then used the Hilbert transform (see Video 6). The phase map shows that (i) the circular waves are slightly off-center from the pillars (ii) the territories that each circular wave propagates before annihilation have different sizes, and (iii) the frequency and phase of the circular waves can vary between the pillars. In Fig. $1 \mathrm{~F}$, we calculated the gradient vectors of the phase map around two neighboring pillars. The vector field changes direction where the concentric waves meet and annihilate each other. Thus, the Laplacian of the phase map $\phi$, defined as $\partial^{2} \phi(x, y) / \partial x^{2}+\partial^{2} \phi(x, y) / \partial y^{2}$, takes on extreme values at the collision regions of two neighboring emitted waves and defines the boundaries of the domains (see Fig. 1G). We also performed 2D Fourier transform of Fig. 1G and looked at the power spectrum. This analysis is presented in Fig. S6A-C which shows dominant peaks corresponding to $5 \mathrm{~mm}$ periodicity of the lattice. Moreover, presentation of power spectrum in polar coordinates shows clear $\pi / 2$-periodicity of the lattice in the presence of caffeine (see Fig. S6C). In contrast, as shown in Fig. S6D-F, in an experiment without caffeine power spectrum shows a wider distribution and $\pi / 2$-periodicity is absent.

Fig. 2A shows the space-time plot along the white dashed line in Fig. 1A. For this kymograph, the processed images in Video 5 are used. First, we observe synchronized bulk oscillations (with a period around $10 \mathrm{~min}$ ). We attribute this to initial starvation of the cells in a shaking suspension for four hours, that leads to cell synchronization (26). After about one hour, circular waves are initiated almost synchronously from the pillars and propagate outwards (see supplemental Fig. S7 and Video 7 for an analysis on the degree of synchronization). The 

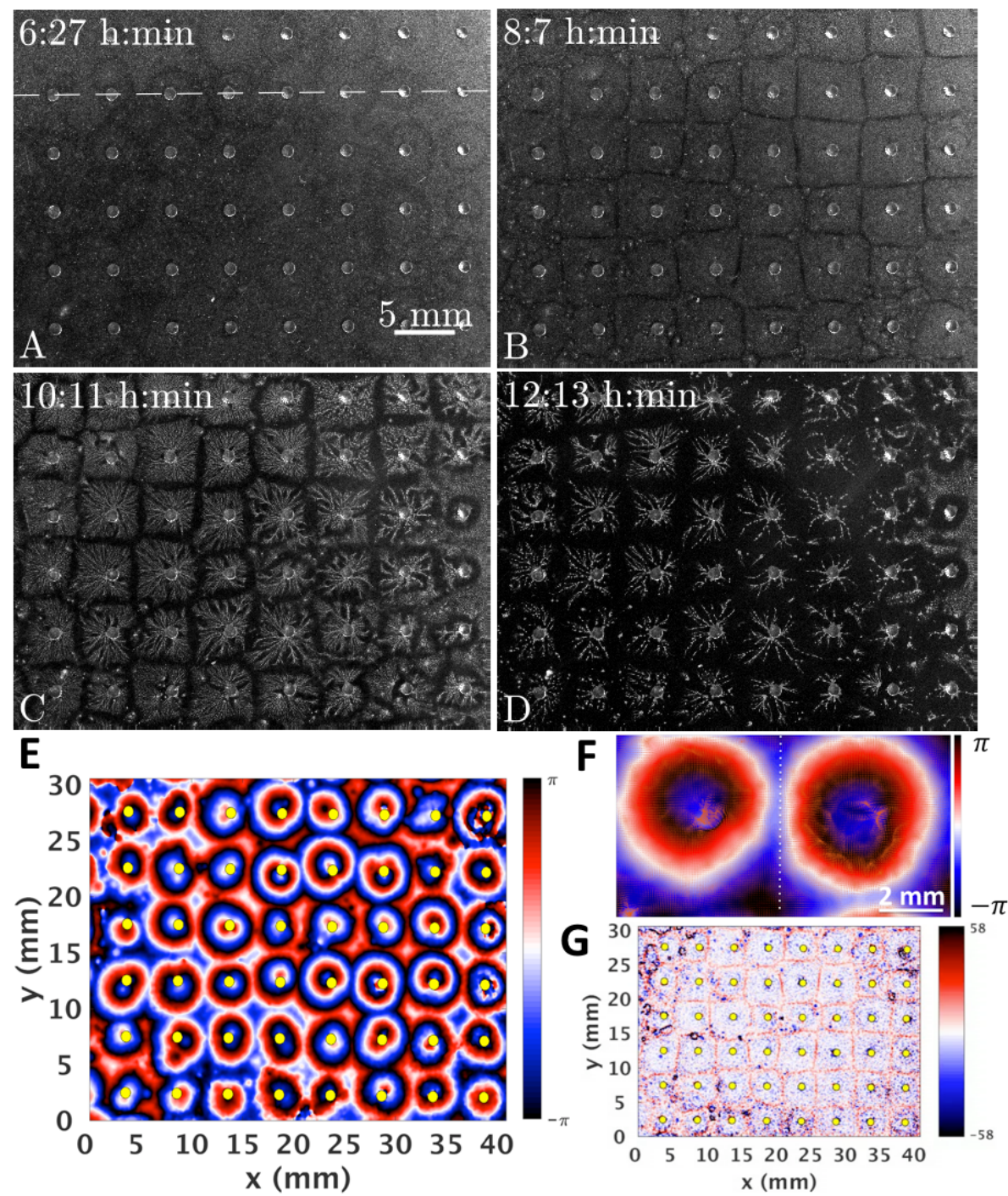

Fig. 1: Formation of regular Voronoi domains. Top view of D.d. cells on a macro-pillar array. A) Concentric waves initiate around the pillars and propagate outwards. (B-D) The amoebas respond chemotactically to the circular waves and stream towards the pillars. This leads to the formation of regular domains around the pillars. Timestamps denote time since start of starvation. E) Phase map of the observed spatio-temporal pattern showing the formation of circular waves around the pillars. F) Gradient vectors of the phase map around two neighboring pillars. The white dashed line shows the location where two emitted waves collide and the vector field changes direction. The vectors are scaled 50 times larger than the original values for better visibility. G) Laplacian of the phase map in unit of $\mathrm{mm}^{-2}$. The gradient vector field switches direction at the boundary of the domains, thus the Laplacian has higher values at these boundaries. 

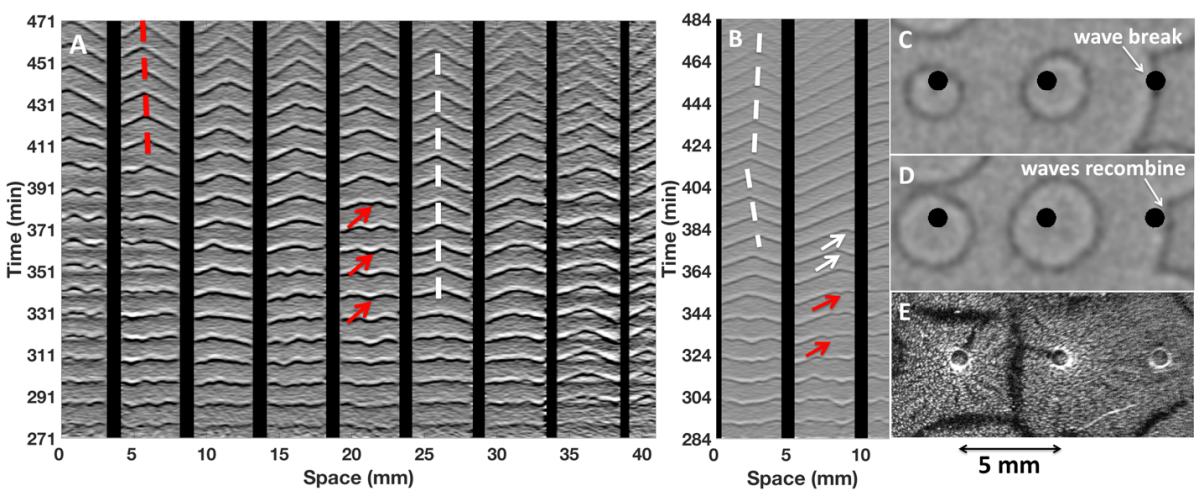

Fig. 2: A) Space-time plot of the experiment in Fig. 1. The light intensity from Video 5 is stacked up along the white dashed line shown in in Fig. 1A. The black bars show the position of the pillars. The red arrows show a firing center other than the pillars that has a lower frequency and disappears with time. The white dashed line traces the annihilation points of two waves that initiate on the neighboring post and have a similar frequency but a phase shift. The red dashed line shows the slow drift of the annihilation point towards the pillar with smaller frequency (left pillar). B) Space-time plot in an experiment with pillars $50 \mu \mathrm{m}$ high. Only part of the kymograph with 3 pillars is shown. The red arrows point to a firing center not centered on a pillar, which has a slightly lower frequency than the waves initiated on the neighboring pillars and thereby disappears with time. The white dashed lines show the movement of the annihilation points towards the pillars with lower wave frequency. The white arrows show the collision of the annihilation point with the right pillar. This pillar fails to emit its own circular cAMP wave and only breaks the wave front initiated from the neighboring pillar $\mathbf{C - E}$ ) As the wave front collides with the pillar on the right, it breaks and recombines again. As a result no boundary between two neighboring pillars forms.

waves annihilate as they collide with each other. These annihilation areas define the boundaries of the regular domains. In an ideal experiment, where the circular waves have the same frequency and phase, the size of the quadratic domains is the same as the pillar spacing $(\sim 5 \mathrm{~mm})$. However, in general there is a frequency and phase difference between concentric waves originating from neighboring pillars consistent with the variety in target centers observed in other excitable systems (27). If two neighboring waves have almost the same frequency with a phase shift, due to this phase difference, the annihilation point of two neighboring circular waves is not located exactly at the middle of two pillars, but is rather located closer to the pillar with a phase delay. This is shown by the white dashed line in Fig. 2A. Moreover, often in the experiments, there is also a small frequency difference between the waves initiating at the neighboring pillars. Consequently, the annihilation point of two neighboring concentric waves shifts towards the pillar with smaller frequency. This event is shown by the red dashed line in Fig. 2A and white dashed lines in Fig. 2B. The drift velocity of the annihilation point can be calculated to be $v_{w}\left(f_{2}-f_{1}\right) /\left(f_{2}+f_{1}\right)$, where $v_{w}$ 

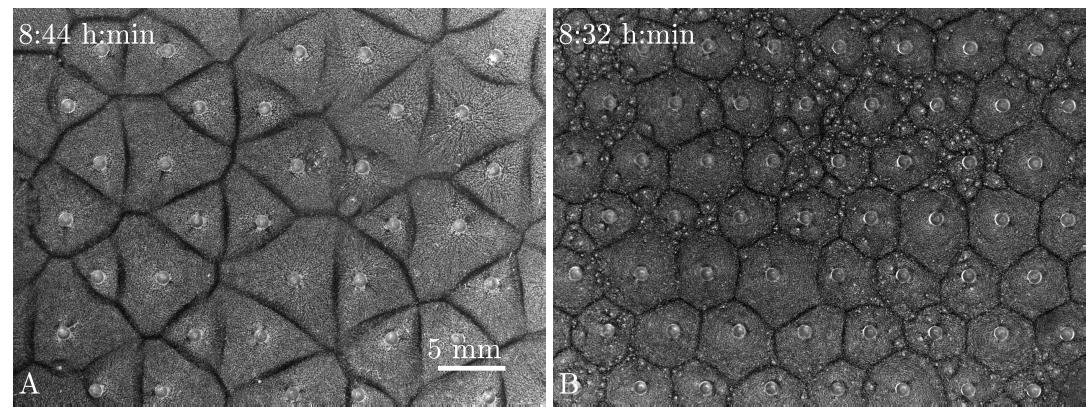

Fig. 3: Different arrangement of the obstacles. Triangular and hexagonal domains formed around the pillars with $\mathbf{A}$ ) hexagonal and $\mathbf{B}$ ) triangular arrangement, respectively.

is the wave propagation velocity and $f_{1}$ and $f_{2}$ are the wave frequencies at the two neighboring pillars (see Fig. S8). In our experiments, $v_{w}$ is of the order of $0.4 \mathrm{~mm} / \mathrm{min}$ and the frequencies $f_{1}$ and $f_{2}$ are roughly $1 / 10 \mathrm{~min}^{-1}$ and $1 / 9$ $\min ^{-1}$, which gives $v_{\text {drift }}$ to be of the order of $0.02 \mathrm{~mm} / \mathrm{min}$. If the frequency difference persists, eventually the wave front collides with the pillar and breaks as is shown by white arrow in Fig. 2C. They recombine after passing through the obstacle and propagate further (Fig. 2D). In this case, the obstacle only breaks the propagating wave front and there is no boundary formed between the middle and the right pillar in Fig. 2E.

The phenomenon of initiation of synchronized circular waves around the pillars and formation of regular domains was robust with respect to the arrangement of the pillars. We repeated our experiments with the same pillar size and spacing, but triangular and hexagonal arrangements of the pillars, and observed hexagonal and triangular domains, respectively (Fig. 3 as well as Video 8 and Video 9).

Next, we used bright field microscopy to look closely at the wave propagation and cell streaming in the vicinity of the pillars (see Fig. 4 and Video 10). A higher cell density around the pillars after plating the cells could explain the higher frequency of the waves initiating from the pillars (see supplemental Fig. S5 and Video 11 that show the effect of local cell density on the wave frequency in a separate experiment). Our extensive bright field observations did not confirm a significant cell accumulation around the pillars (see Fig. 4E). However, based on our numerical simulations, in the presence of caffeine even a slight cAMP accumulation in the vicinity of the obstacles is enough to trigger formation of concentric waves around the pillars. Possible cell attachment to the side walls of the pillars which is simulated as a small constant value of cAMP on the obstacles, is a plausible mechanism for the formation of circular waves around the pillars. Moreover, we emphasize that initial starvation of the cells for 4 hours in a shaken suspension leads to increased cell-cell adhesion, and very small cell clusters are visible in Fig. 4A. As we mentioned before, concentric waves around the pillars are not always completely centered at the pillars, but are slightly off-center. Therefore, the cells first stream towards a point in the vicinity of the pillar (arrow in Fig. 4B and Video 10) and then aggregate at 

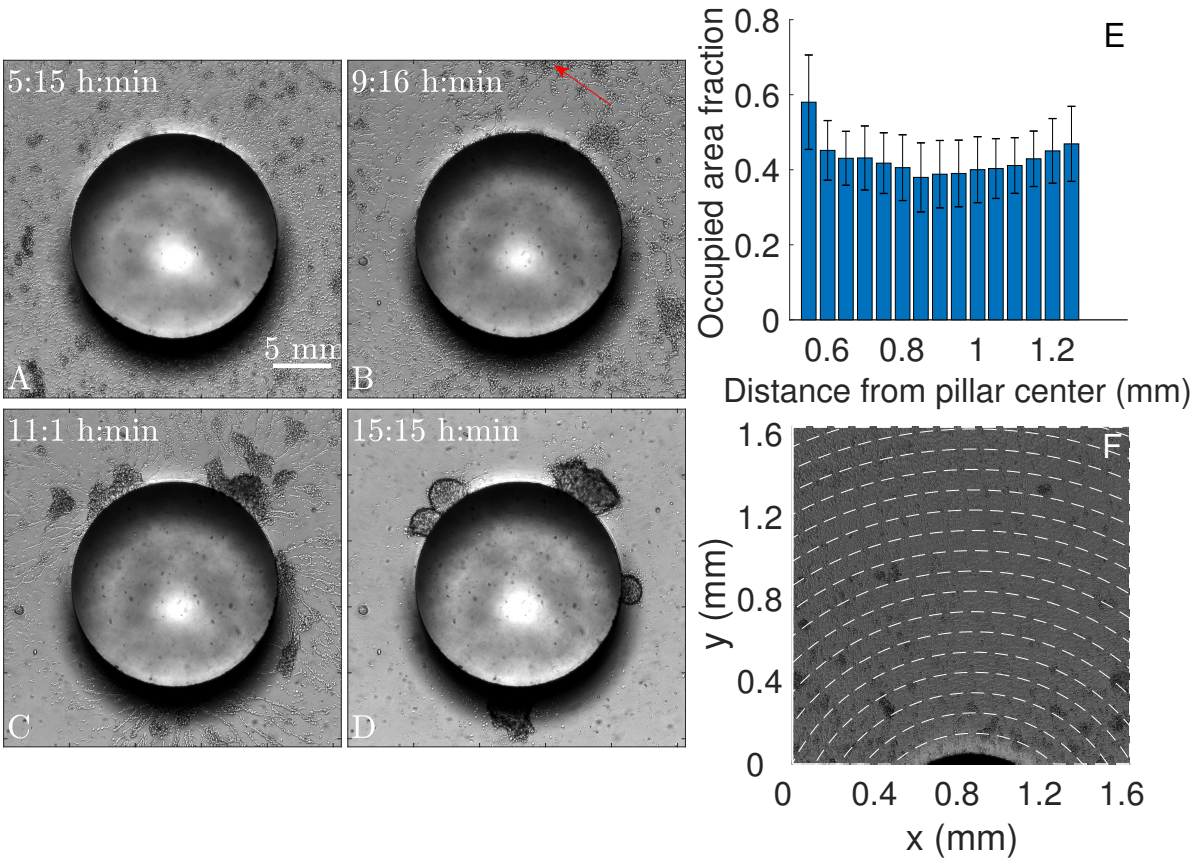

Fig. 4: Cell distribution around the pillars. A-D) A closer look at the pillars with a bright field microscope. A) Initial distribution of the cells show small clumps. B-D) Cells chemotactically move towards the center of the concentric waves, which are sometimes slightly off-center from the pillars. During the streaming process, the cells join small clusters to make larger ones and eventually large clusters aggregate on the post (see also Video 10). E-F) Systematic measurements of density around the pillars of diameter $1 \mathrm{~mm}$ show no significant cell accumulation in the vicinity of the obstacles. The distance is measured from center of the pillar and standard deviations are calculated from the measurements for $\sim 11$ pillars. Note that the area around each pillar is divided to circular bands and the occupied area fraction is measured for each band, as shown in $\mathbf{F}$.

the pillar itself. Note that in our typical experiments which are done with 20 $\mathrm{ml}$ cell solution (see Methods and Materials), the depth of fluid is around 4.5 $\mathrm{mm}$. Therefore cells aggregate on the pillars but fail to form fruiting bodies as the air interface is relatively far away. To allow for fruiting bodies to form, we performed a new set of experiments with $2 \mathrm{ml}$ of cell solution (but the same number of cells/area) to reduce the height of fluid to $400 \mu \mathrm{m}$. As shown in supplemental Fig. S9 and Video 12, regular streaming domains emerge and cells aggregate on the obstacles, eventually forming fruiting bodies on the pillars and in the area in a close vicinity of the pillars. Note that in this experiment pillars of height $50 \mu \mathrm{m}$ are used to avoid fluid meniscus around the pillars and the diameter of pillars is $1 \mathrm{~mm}$.

In nature obstacles encountered by D.d. cells are smaller in size and have a random distribution. Thereby, to better approximate natural habitat of the 
cells, we performed multiple sets of experiments with 1) pillars of random diameter $(0.1 \leq d \leq 1 \mathrm{~mm})$ distributed randomly on a PDMS substrate, 2$)$ pillars of diameter $1 \mathrm{~mm}$ distributed randomly and 3) pillars of diameter $100 \mu \mathrm{m}$ in a periodic lattice. These results are presented in supplemental Fig. S10 as well as Video 13, Video 14 and Video 15. Interestingly, pillars of smallest diameter of $100 \mu \mathrm{m}$ mostly become wave centers and cells stream towards the obstacles (see Fig. S10E-F). However, as we further reduced the pillar diameter to $50 \mu \mathrm{m}$, the obstacles were mostly ignored by the cells suggesting a minimum obstacle size for pillars to act as a wave center. Moreover, in the case of random pillar distribution, we observed that two pillars located in a close vicinity of each other effectively act as one wave center (see Fig. S10D) which suggests that an optimal distance between obstacles is needed such that each pillar successfully emits its own circular wave and becomes an aggregation center.

\section{Numerical Simulations}

We performed numerical simulations of the model proposed by Martiel and Goldbeter (MG) for the creation and relay of cAMP in D.d. (28, 29). Many approaches have been used to create spirals and target centers in this model, most of which define the position of the localized structures through perturbations or diversity of developmental stage among the cells $(30,31)$. We instead used a dynamical approach where centers appear naturally in areas of higher local density (32) (see Methods and Materials and Video 16). Here, the cell locations are discretized inside grids, thus a grid containing a cell is an occupied one and can produce and relay cAMP, while the empty grids without cells can only degrade the signal via external phosphodisterase (PDE). This mixture of occupied and unoccupied areas on the system breaks the system homogeneity and allows clusters of local higher cell density to become target centers. The lower density areas are still capable of sustaining waves, thus the waves generated by these clusters get relayed by the rest of the system. We measured the dispersion relation of such waves and showed that they have the behavior of trigger waves (33) (see Fig. 5). These dispersion relations at different cell densities also showed that the wave velocity increases with cell density, which has been reported to be necessary to produce aggregation streams (34). Note that trigger waves are nonlinear excitation waves that propagate in excitable media when a perturbation above a threshold is applied. In these systems, small perturbations damp out but supra-threshold ones are amplified and excite the neighboring area allowing for wave propagation. A trigger wave has a velocity which is nonlinearly selected by the system and both the propagation speed as well as its profile are uniquely determined by the properties of the medium. Another important characteristic is that a new trigger wave cannot enter the system until some recovery time has elapsed. Finally, we emphasize that trigger waves should be distinguished from phase waves that exist due to phase gradients between coupled oscillators and unlike phase waves, trigger waves present actual chemical transport. Phase waves are largely independent of diffusion, and have a variable speed, which is not intrinsic to the system and can be arbitrarily large (35).

We observed in our simulations that a no flux boundary condition at the obstacles is not sufficient to produce centers. Similarly, when this boundary condition is applied, waves only break after colliding with the obstacles and recombine again (see Fig. S11 and Video 17). This was in agreement with 

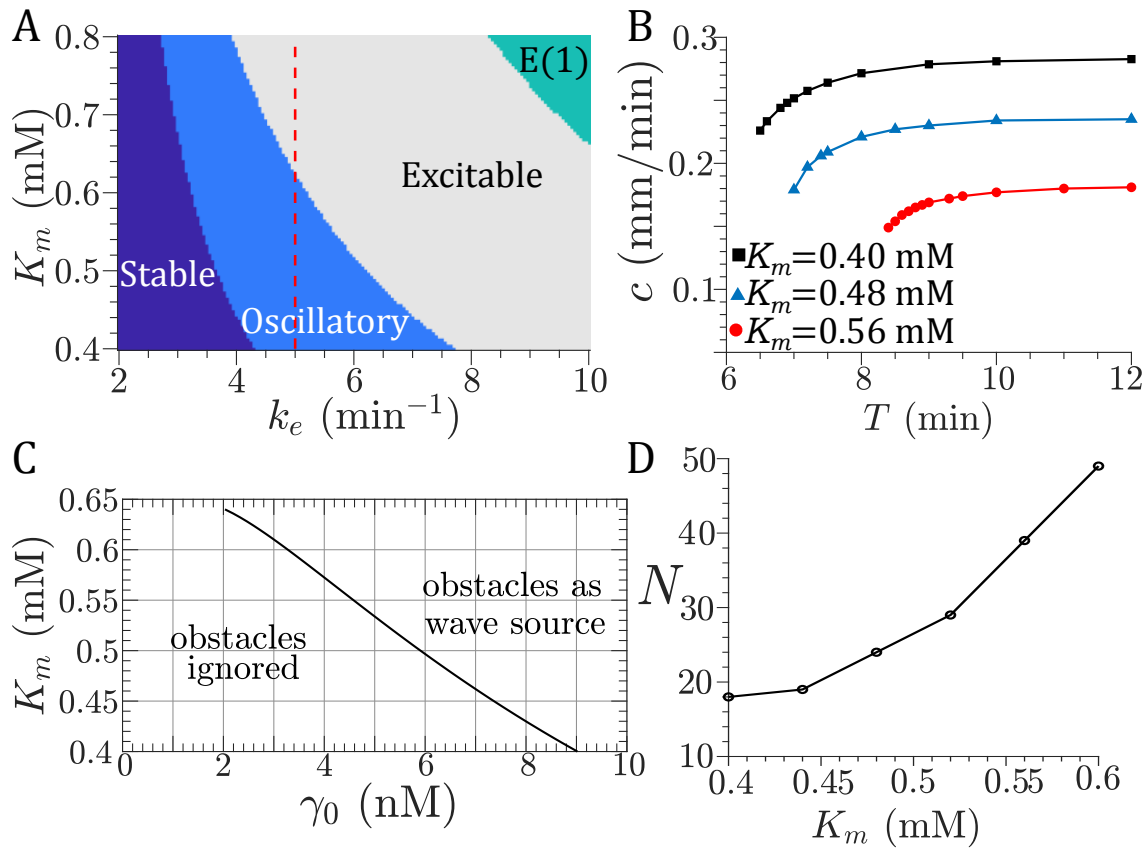

Fig. 5: Characterization of the MG model with obstacles. A) Phase diagram of the MG model used for simulating the experimental setup. In the area marked as Stable, the system has one solution, which is stable. In the Oscillatory region, the system shows one unstable steady state surrounded by a limit circle. In the Excitable regime, the system has 3 steady states, two unstable ones and a stable one, which is excitable. In the regime marked as $\mathrm{E}(1)$ the system shows one steady state, which is excitable. Stability calculated through linear analysis, and excitability through no-space simulations. Red dashed line shows the path that $K_{m}$ is changed in parts $\mathbf{B}, \mathbf{C}$ and $\mathbf{D}$ at fixed value of $k_{e}=5.0 \mathrm{~min}^{-1}$. Note that higher $K_{m}$ values correspond to higher caffeine concentration. B) Dispersion relations of the supported wave-trains for $40 \%$ surface coverage, where $T$ is wave period and $c$ wave velocity. Waves with periods below those shown, do not get relayed by the system. C) Effect of a boundary condition $\gamma_{0}$ : for higher values of $K_{m}$ the minimum amount of $\gamma_{0}$ required to produce a target center decreases. D) Minimum number $N$ of consecutive cells (in 1-D) needed to produce a cluster with self-sustained oscillations.

our initial experiments without caffeine. Another boundary condition that has been shown to produce traveling waves in numerical simulations is the Dirichlet boundary condition, where the boundary is held to a fixed value. In our system, a fixed value of cAMP at the obstacles creates wave trains emitted from the obstacles if the fixed value is larger than a threshold, as it is shown in Fig. 6A-B) and Video 18. This minimum amount of cAMP needed for the obstacles to act as a wave source depends on the system parameters. The minimum accumulation of cAMP or cells necessary for an unexcitable obstacle to become a center is high enough so that the cells do not aggregate around the obstacles natu- 
rally. In order to obtain aggregation domains around the obstacles, we varied different parameters, which can be controlled in our experiments. To achieve a lower excitation threshold, we varied the parameter $K_{m}$ which is the Michaelis constant of the reaction in which ATP produces intracellular cAMP. Increasing this parameter reduces the affinity between ATP and the enzyme adenylate cyclase (ACA), thus reducing the production rate of intracellular cAMP and accounting for the effects of adding caffeine to the experimental system. We performed linear stability analysis of the MG model with modifications in $K_{m}$ and characterized its different regimes, which are shown in Fig. 5A, the other parameter we varied is the degradation rate of external phosphodiesterase $k_{e}$. We chose the parameters such that the system is in the oscillatory regime, i.e. a stable limit cycle exists, and the cell coverage (ratio of occupied grids to total number of grids) is high enough for the waves to get relayed. At higher values of $K_{m}$, which is equivalent to higher concentration of caffeine in our experiments, the number of firing centers in the system is decreased (Fig. S12 and Video 19). The reason is that the minimum cluster size (measured as consecutive cells in a 1D setting) required to produce a self-sustained oscillatory center is increased (see Fig. 5D). Increasing the amount of caffeine (higher $K_{m}$ values) also decreased the wave velocity and increased the minimum wave period the system can sustain. Different dispersion relations showing this effect are depicted in Fig. 5B. We studied the boundary effect in this system and found that at higher caffeine concentration the trigger waves require a smaller amount of cAMP to be triggered, thus the obstacles act as a wave source at smaller values of the fixed boundary $\gamma_{0}$ (see Fig. $5 \mathrm{C}$ and Video 18).

Another mechanism for wave creation observed in our simulations was a higher local cell density around the pillars. This can be achieved either by inhomogeneous initial cell distribution or by adhesion of the cells to the pillars after colliding due to random movement. These locally high density cell clusters trigger the formation of wave centers and act as aggregation centers, as shown in Fig. 6C-D and Video 20. Notably, we also performed numerical simulations with hexagonal and triangular arrangement of the pillars (Dirichlet boundary condition) and observed triangular and hexagonal patterns, respectively (see Fig. 6E-H with corresponding videos Video 21 and Video 22).

Finally, it is experimentally suggested that the effect of caffeine on ACA inhibition is possibly mediated by different targets, one of which inhibits the activation of G-protein G2, that is part of the signal transduction cascade that normally activates ACA (36). To investigate the effect of having less activated ACA, we also performed simulations by modifying the parameter $\epsilon$ which controls the ratio of the active to inactive forms of ACA. These simulations showed similar results to those obtained by modifying $K_{m}$ and are presented in the Supplementary Material. Therefore, regardless of the particular way in which caffeine affects ACA, our numerical results provide explanation for the observed behavior (see Fig. S13, Fig. S14 and Video 23).

\section{Discussion}

The results presented here show that external obstacles can significantly influence the generation of waves in starving populations of D.d. cells when the excitability threshold has been lowered. We observed circular waves that initiate 

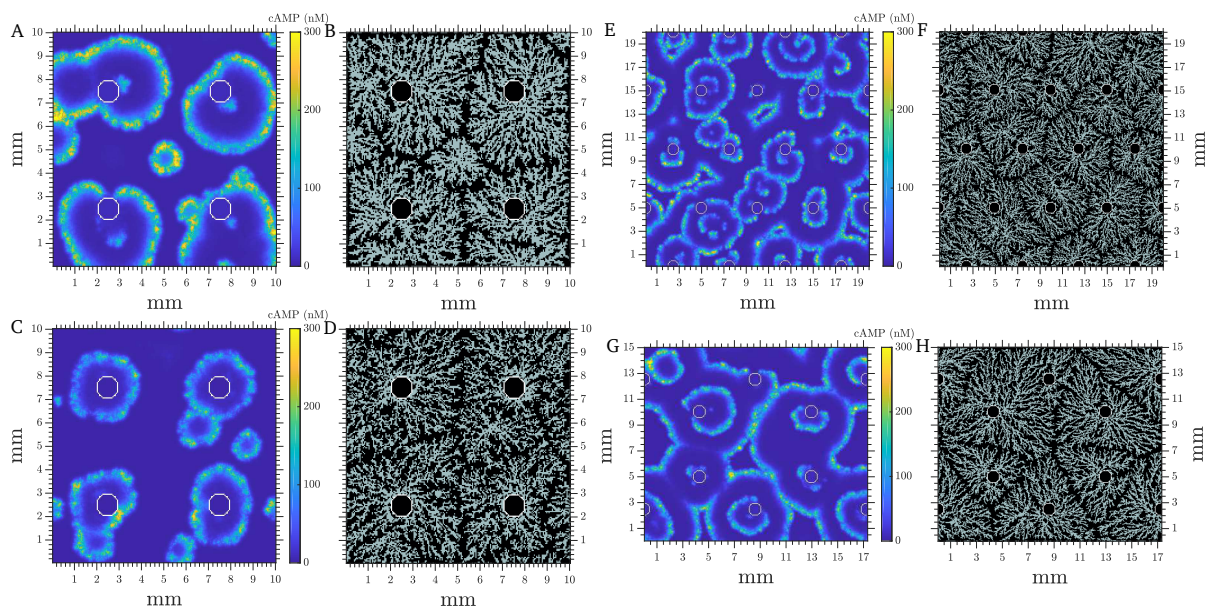

Fig. 6: Numerical simulations of the reaction-diffusion model. A-B) Numerical simulations of cAMP waves with four pillars as obstacles with fixed boundary condition $\gamma_{0}=10 \mathrm{nM}, K_{m}=0.508 \mathrm{mM}, k_{e}=5.0 \mathrm{~min}^{-1}, 50 \%$ of cell surface coverage. A) Concentric waves of cAMP coming out of the pillars. B) Cell distribution after $t=100 \mathrm{~min}$, showing regular domains around the pillars. Grey squares show grid points containing a cell, black squares show the empty ones. C-D) Numerical simulation showing the effect of a higher cell accumulation around pillars. $K_{m}=0.48 \mathrm{mM}, k_{e}=5.0 \mathrm{~min}^{-1}, 60 \%$ of cell coverage around the pillar, $40 \%$ in the rest of the system. C) Waves of cAMP at $t=15 \mathrm{~min}$. D) Cell distribution after $t=150 \mathrm{~min}$. Grey squares show grid points containing a cell, black squares show the empty ones (see also Video 20). E, G) Numerical simulations of cAMP waves with a triangular and hexagonal pillar array showing concentric waves of cAMP coming out of the pillars. Fixed boundary condition $\gamma_{0}=10 \mathrm{nM}, K_{m}=0.508 \mathrm{mM}, k_{e}=5.0 \mathrm{~min}^{-1}, 40 \%$ of cell surface coverage. $\mathbf{F}, \mathbf{H})$ Cell distribution after $t=120 \mathrm{~min}$ and $t=150$ min, respectively, showing regular domains around the pillars (see Video 21 and Video 22).

almost synchronously at the pillars and propagate outwards. Chemotactically competent cells detect the cAMP gradient and crawl towards the pillars, forming a periodic array of domains. This phenomenon is also observed for triangular and hexagonal arrangement of the pillars leading to the formation of hexagonal and triangular domains, respectively. Most of our experiments were performed with pillars of $1 \mathrm{~mm}$ diameter, but the phenomenon showed to be fairly robust to different types of obstacles, since we observed a similar phenomenon with pillars of $1.5 \mathrm{~mm}$ diameter (see Video 24), random distribution of the pillars, random pillar diameters, pillars of diameter $100 \mu \mathrm{m}$, shallow holes $(100 \mu \mathrm{m}$ in depth) in PDMS (see Video 25), smaller center-to-center spacing of the pillars (3.75 $\mathrm{mm}$ instead of $5 \mathrm{~mm}$ ), and even with a PDMS wall (see Video 26). The phenomenon is also robust with respect to the pillar height since we observed regular domains with pillar's height down to 50 micron (see Fig. 2B-E and Video 27).

The concentric waves emitted from pillars have a slightly higher frequency 
than the other firing centers, thereby dominating the system dynamics. Cells attached to the side walls of the PDMS can trigger higher frequency waves. In our simulations, we included this effect by assuming either a fixed value of cAMP around the pillars or slightly higher cell accumulation in the vicinity of pillars. Future experiments using fluorescent indicators for extracellular cAMP, will be valuable to visualize any possible cAMP accumulation around the pillars $(37,38)$. Another scenario which requires extra bio-chemical analysis, is the possibility of accumulation or depletion of any chemical (such as phosphodiestrase) in the vicinity of the pillars. Our numerical simulations show that in the case of PDE depletion, pillars can act as wave source in the system, that might drive oscillations in the vicinity of the obstacles (39-41) (see Video 28). In the opposite case of PDE accumulation, pillars are "ignored" and only break the colliding wave fronts (see Video 29). However, we should mention that, to prevent adsorption of chemicals to the PDMS substrate, we repeated our experiments with BSA (Bovine Serum Albumin) treated substrates and still observed concentric waves and periodic domains around the pillars. Finally, although PDMS is useful because of its bio-compatibility, deformability and permeability to gas, it has certain properties which might complicate our results. To rule this out, we used pillars made of PMMA and again observed the regular streaming domains.

In our system, we observed periodic domains within a range of caffeine concentration between 1-5 mM, when cells are initially synchronized in an agitated starving period. Caffeine, which is a highly specific inhibitor of cAMP relay (42-45), reduces both the cAMP production rate and wave frequency in a dosedependent manner (see Fig. S15). We did not observe ordered aggregation territories without caffeine (see Fig. S3A-B and Video 2). Our experiments and earlier experiments by (46) with caffeine have shown that the aggregation territories are much larger in the presence of caffeine compared to the control experiments (see Fig. S16 and Video 30). This means that the number of firing centers is decreased, which is also consistent with our numerical simulations in the presence of caffeine with higher values of $K_{m}$ (Fig. S12 and Video 19). We believe that in our experiments, a lower number of firing centers is crucial for circular waves emitted from pillars to successfully take over and dominate the system dynamics $(39,47,48)$. We also observed that in the presence of obstacles, caffeine drastically diminished the number of spirals appearing in the system. Since spirals have a higher frequency than target patterns, they take over and dominate the dynamics once they appear. Moreover, we emphasize that in our experiments without initial starvation of the cells in an agitated suspension, mostly spirals form confirming that synchronization level of cells plays an important role in shaping the overall wave pattern; see Video 31. In other excitable systems, such as the BZ reaction it has been shown that spirals can be pinned to obstacles with lower excitability (13). In that reaction-diffusion system it has also been shown numerically that the spiral tip interacts with the (no flux) obstacle boundary through attraction and repulsion (17). We observe neither attraction nor repulsion of the spiral cores to the obstacles in both the experimental setup and the numerical simulations. We believe this crucial difference is given by the lack of meandering of the spiral tip in D.d. Interestingly, it has been shown that in the presence of caffeine spiral core size increases in a dose-dependent manner (44). In those occasions when a spiral did appear next to a pillar, it got pinned to the pillar and remained rotating around it (see Fig. 
S2 and Video 1).

Comparing our numerical simulations with modified $K_{m}$ to the unmodified parameters, the system showed fewer centers, due to higher minimum cluster size necessary to produce pacemakers; it also showed smaller frequency, in agreement with experimental measurements (see Fig. S15). The minimum amount of cAMP necessary to produce cell activation was diminished, allowing for easier wave relay. This is consistent with experimental results in (42) showing that caffeine indeed increases apparent chemotactic sensitivity of the cells. Thus, in the presence of caffeine, the transition boundary between "source" and "ignored" obstacles occurs at lower values of cAMP accumulation around the obstacles. For this reason, the presence of caffeine is necessary in our experiments to trigger formation of concentric waves around the pillars. Since in nature an inhomogeneous distribution of cells and physical obstacles is expected, we believe that a combination of two factors, namely the chemotactic sensitivity and synchronization level in a population of signaling cells strongly influence the interaction between cells and obstacles.

\section{Methods and Materials}

\section{Experimental Methods}

The D.d. cells (strain AX2-214) were grown at $22^{\circ} \mathrm{C}$ in HL5 medium, harvested in the exponential growth phase, and starved for four hours in $10 \mathrm{~mL}$ phosphate buffer supplemented with $2 \mathrm{mM}$ caffeine in a shaking suspension. After 4 hours of starvation, they were centrifuged and diluted to a density of $2 \times 10^{6} \mathrm{cells} / \mathrm{ml}$ in fresh phosphate buffer containing $2 \mathrm{mM}$ caffeine. Next, $20 \mathrm{~mL}$ cell solution $\left(\sim 0.9 \times 10^{6}\right.$ cells $/ \mathrm{cm}^{2} \sim 0.9$ mono-layer $)$ was transfered to a modified Petri dish with a plasma-treated polydimethylsiloxane (PDMS) substrate (49). The PDMS has a periodic array of macro-pillars characterized by pillar dimensions and spacing (see Fig. S1). If not stated otherwise, pillars of $1 \mathrm{~mm}$ diameter and height of $3 \mathrm{~mm}$ are arranged on square, triangular or hexagonal lattices with lattice size of $5 \mathrm{~mm}$. cAMP wave patterns are indirectly visualized by dark-field microscopy $(10,50,51)$. Note that under dark-field illumination the light scattered by the cells is a measure of changes in the cell shape and indirectly reflects the concentration of cAMP. Direct visualization of cAMP waves using isotope dilution-flourographic technique in Ref. (52) has shown that cell shape change and concentration of cAMP are indeed correlated. While at low cAMP concentrations, cells are more or less round and scatter a little light, at high concentrations of cAMP, chemotactic cells are elongated and scatter more light. Pairs of darkfield images, separated by 1 minute are subtracted from one another, then bandpass filtered to reduce spatial noise. These filtered images are used to obtain phase maps for visualizing wave propagation in oscillatory systems. They are obtained using a mathematical operation called the Hilbert transform which converts a real signal to an analytical signal with real and imaginary parts. The phase angle of this analytical signal is then between $[-\pi, \pi]$ and represents the phase of the oscillatory system. Waves propagate in regions where the phase field is continuous, and in standard convention, in the direction from positive to negative phase. Note that in the areas where the waves collide the phase field is no longer continuous. 


\section{Numerical Methods}

The reaction-diffusion equations used for modeling this system are

$$
\begin{aligned}
k_{1}^{-1} \partial_{t} \rho_{i} & =-f_{1}\left(\gamma\left(x_{i}, y_{i}\right)\right) \rho_{i}+f_{2}\left(\gamma\left(x_{i}, y_{i}\right)\right)\left(1-\rho_{i}\right), \\
\partial_{t} \beta_{i} & =s \Phi\left(\rho_{i}, \gamma\left(x_{i}, y_{i}\right)\right)-\left(k_{i}+k_{t}\right) \beta_{i} \\
\partial_{t} \gamma & =D \nabla^{2} \gamma-k_{e} \gamma+\sum_{i}^{N} H(i, x, y) k_{t} \beta_{i} / h
\end{aligned}
$$

with $f_{1}(\gamma)=\frac{1+\kappa \gamma}{1+\gamma}, f_{2}(\gamma)=\frac{\mathcal{L}_{1}+\kappa \mathcal{L}_{2} c \gamma}{1+c \gamma}, \Phi(\rho, \gamma)=\frac{\lambda_{1}+Y^{2}}{\lambda_{2}+Y^{2}}, Y(\gamma, \rho)=$ $\frac{\rho \gamma}{1+\gamma}$ and $s=q \sigma \alpha /(1+\alpha)$, where $\gamma(x, y)$ and $\beta_{i}$ are the amount of extracellular and intracellular cAMP respectively. $\rho_{i}$ corresponds to the percentage of active cAMP receptors on the cell surface and acts effectively as the slow variable that gives the system its excitable capabilities. $k_{e}$ corresponds to the extracellular phosphodiesterase (PDE) and $s$ controls the amount of cAMP produced inside the cells. $H(i, x, y)$ is an index variable with values 1 if the $i-t h$ cells is located in $(x, y)$ and 0 if it is not. This produces that on the grid points containing amoebas the cAMP is transported from the intra- to the extracellular media, while on the empty cell spaces the wave is degraded by the unbounded phosphodiesterase. The systems was simulated using discrete differences, a 5 point laplacian, and a time adaptive Runge-Kutta scheme. The used parameters were $\sigma=0.55$ $\min ^{-1}, k_{1}=0.09 \min ^{-1}, \kappa=18.5, \mathcal{L}_{1}=10, \mathcal{L}_{2}=0.005, c=10, q=4000$, $\alpha=1.2 / K_{m}, \lambda_{1}=10^{-4} / \epsilon, \lambda_{2}=0.2575 / \epsilon, k_{i}=1.7 \mathrm{~min}^{-1}, k_{t}=0.9 \mathrm{~min}^{-1}$, $D=0.024 \mathrm{~mm}^{2} / \mathrm{min}$, and $h=5$. For simulating the effects of caffeine either $K_{m}=0.4-0.6 \mathrm{mM}$ and $\epsilon=1.0$ or $K_{m}=0.4 \mathrm{mM}$ and $\epsilon=0.6-1.0$ are used. Note that higher $K_{m}$ values correspond to higher concentrations of caffeine, while higher values of $\epsilon$ is a measure of lower caffeine concentrations (see Video 23 for a simulation of different values of $\epsilon$ ). With these parameters the system is in an oscillatory state, meaning that a limit cycle exists. If a cluster of cells of big enough size exists, it acts as a pacemaker producing trigger (chemical) waves that are relayed by the system, as long as a minimum percentage (measured as surface coverage) of cells exists. For a description of how this model produces target centers see (32). Emulating the experimental observations, after some simulation time, when the waves have been established, we allowed the cells to be chemotactically competent. The movement rules were as follows. If a cell detects a cAMP gradient bigger than a threshold $\left(\nabla \gamma>g_{t h}\right)$ and it is in the excitable state $\left(\rho>\rho_{t h}\right)$, it moves against the gradient with a velocity $v_{c}$ as long as the two previous conditions continue to be fulfilled. If the new position falls in a different grid space, the movement occurs only if the new grid point does not already contain a cell. The parameters used were $g_{t h}=25.98 \mathrm{nM} / \mathrm{mm}$, $\rho_{t h}=0.6, v_{c}=20 \mu \mathrm{m} / \mathrm{min}$, and we allowed cell movement after $t=50 \mathrm{~min}$.

\section{References}

(1) A. V. Holden, M. Markus, and H. G. Othmer. Nonlinear wave processes in excitable media. Vol. 244. Springer, 2013. 
(2) P. Grindrod. Patterns and waves: The theory and applications of reactiondiffusion equations. Oxford University Press, USA, 1991.

(3) E. Meron. "Pattern formation in excitable media". In: Physics Reports 218.1 (1992), pp. 1 -66. ISSN: 0370-1573. DOI: https://doi .org/10. 1016/0370-1573(92) 90098-K.

(4) V. S. Zykov. Simulation of wave processes in excitable media. Manchester Univ Press, Manchester, UK, 1987.

(5) A. N. Zaikin and A. M. Zhabotinsky. "Concentration Wave Propagation in Two-dimensional Liquid-phase Self-oscillating System". In: $\mathrm{Na}$ ture 225.5232 (1970), pp. 535-537. DOI: https://doi .org/10.1038/ $225535 b 0$.

(6) R. Imbihl and G. Ertl. "Oscillatory Kinetics in Heterogeneous Catalysis". In: Chemical Reviews 95.3 (May 1995), pp. 697-733. DoI: https://doi. $\mathrm{org} / 10.1021 / \operatorname{cr} 00035 \mathrm{a} 012$.

(7) N. A. Gorelova and J. Bureš. "Spiral waves of spreading depression in the isolated chicken retina". In: Journal of Neurobiology 14.5 (1983), pp. 353363. DOI: https://doi.org/10.1002/neu. 480140503.

(8) J. M. Davidenko, A. V. Pertsov, R. Salomonsz, W. Baxter, and J. Jalife. "Stationary and drifting spiral waves of excitation in isolated cardiac muscle". In: Nature 355.6358 (1992), pp. 349-351. DOI: https://doi . org/10.1038/355349a0.

(9) G. Gerisch. "Stadienspezifische Aggregationsmuster bei Dictyostelium discoideum". In: Wilhelm Roux' Archiv für Entwicklungsmechanik der Organismen 156.2 (1965), pp. 127-144. ISSN: 1432-041X. DOI: https : // doi.org/10.1007/BF00573870.

(10) P. N. Devreotes, M. J. Potel, and S. A. MacKay. "Quantitative analysis of cyclic AMP waves mediating aggregation in Dictyostelium discoideum". In: Developmental Biology 96.2 (1983), pp. 405 -415. DoI: https://doi. org/10.1016/0012-1606 (83)90178-1.

(11) A. V. Panfilov and J. P. Keener. "Effects of High Frequency Stimulation on Cardiac Tissue with an Inexcitable Obstacle". In: Journal of Theoretical Biology 163.4 (1993), pp. 439 -448. ISSN: 0022-5193. DOI: https: //doi.org/10.1006/jtbi.1993.1129.

(12) M. Bär, E. Meron, and C. Utzny. "Pattern formation on anisotropic and heterogeneous catalytic surfaces". In: Chaos: An Interdisciplinary Journal of Nonlinear Science 12.1 (2002), pp. 204-214. DOI: https://doi.org/ $10.1063 / 1.1450565$.

(13) O. Steinbock and S. Müller. "Chemical spiral rotation is controlled by light-induced artificial cores". In: Physica A: Statistical Mechanics and its Applications 188.1 (1992), pp. 61 -67. ISSN: 0378-4371. DOI: https: //doi.org/10.1016/0378-4371 (92)90253-M.

(14) K. H. ten Tusscher and A. V. Panfilov. "Wave propagation in excitable media with randomly distributed obstacles". In: Multiscale Modeling $\&$ Simulation 3.2 (2005), pp. 265-282. 
(15) S. Luther, F. H. Fenton, B. G. Kornreich, A. Squires, P. Bittihn, D. Hornung, M. Zabel, J. Flanders, A. Gladuli, L. Campoy, et al. "Lowenergy control of electrical turbulence in the heart". In: Nature 475.7355 (2011), p. 235. DOI: 10.1038/nature10216.

(16) A. M. Pertsov, J. M. Davidenko, R. Salomonsz, W. T. Baxter, and J. Jalife. "Spiral waves of excitation underlie reentrant activity in isolated cardiac muscle." In: Circulation research 72.3 (1993), pp. 631-650.

(17) A. P. Muñuzuri, V. Pérez-Muñuzuri, and V. Pérez-Villar. "Attraction and repulsion of spiral waves by localized inhomogeneities in excitable media". In: Phys. Rev. E 58 (3 1998), R2689-R2692. DoI: https://doi. org/10.1103/PhysRevE.58.R2689.

(18) D Pazó, L. Kramer, A. Pumir, S. Kanani, I. Efimov, and V. Krinsky. "Pinning force in active media". In: Physical review letters 93.16 (2004), p. 168303.

(19) P. Bittihn, A. Squires, G. Luther, E. Bodenschatz, V. Krinsky, U. Parlitz, and S. Luther. "Phase-resolved analysis of the susceptibility of pinned spiral waves to far-field pacing in a two-dimensional model of excitable media". In: Philosophical Transactions of the Royal Society A: Mathematical, Physical and Engineering Sciences 368.1918 (2010), pp. 22212236 .

(20) V. Krinsky. "Excitation wave propagation during heart fibrillation". In: Biological and biochemical oscillators. Elsevier, 1973, pp. 329-341.

(21) A. Pertsov and A. Grenadier. "The autowave nature of cardiac arrhythmias". In: Self-Organization Autowaves and Structures Far from Equilibrium. Springer, 1984, pp. 184-190.

(22) R. L. Chisholm and R. A. Firtel. "Insights into morphogenesis from a simple developmental system". In: Nature Reviews Molecular Cell Biology 5 (2004), pp. 531-541. DOI: https://doi.org/10.1038/nrm1427.

(23) S. Saran, M. E. Meima, E. Alvarez-Curto, K. E. Weening, D. E. Rozen, and P. Schaap. "cAMP signaling in Dictyostelium". In: Journal of Muscle Research \& Cell Motility 23.7 (2002), pp. 793-802. DOI: https://doi. org/10.1023/A:1024483829878.

(24) D. Proverbio and M. Maggiora. "Dynamical strategies for obstacle avoidance during Dictyostelium discoideum aggregation: a Multi-agent system model". In: arXiv preprint arXiv:1905.05030 (2019).

(25) I. Hecht, H. Levine, W.-J. Rappel, and E. Ben-Jacob. "Self-assisted amoeboid navigation in complex environments". In: PloS one 6.8 (2011), e21955.

(26) G. Gerisch. "Cell aggregation and differentiation in Dictyostelium". In: Current topics in developmental biology. Vol. 3. Elsevier, 1968, pp. 157197.

(27) Y. Kuramoto. Chemical oscillations, waves, and turbulence. Vol. 19. Springer Science \& Business Media, 2012.

(28) J.-L. Martiel and A. Goldbeter. "A Model Based on Receptor Desensitization for Cyclic AMP Signaling in Dictyostelium Cells". In: Biophysical Journal 52.5 (1987), pp. 807 -828. DOI: https://doi .org/10.1016/ S0006-3495 (87) 83275-7. 
(29) J. J. Tyson, K. A. Alexander, V. Manoranjan, and J. Murray. "Spiral waves of cyclic amp in a model of slime mold aggregation". In: Physica D: Nonlinear Phenomena 34.1 (1989), pp. 193 -207. ISSN: 0167-2789. DOI: https://doi.org/10.1016/0167-2789(89)90234-0.

(30) J. Lauzeral, J. Halloy, and A. Goldbeter. "Desynchronization of cells on the developmental path triggers the formation of spiral waves of cAMP during Dictyostelium aggregation". In: Proceedings of the National Academy of Sciences 94.17 (1997), pp. 9153-9158. Dor: https://doi .org/10 . 1073/pnas.94.17.9153.

(31) D. Geberth and M.-T. Hütt. "Predicting the Distribution of Spiral Waves from Cell Properties in a Developmental-Path Model of Dictyostelium Pattern Formation". In: PLOS Computational Biology 5.7 (July 2009), pp. 1-10. DOI: https://doi.org/10.1371/journal.pcbi.1000422.

(32) E. Vidal-Henriquez and A. Gholami. "Spontaneous center formation in Dictyostelium discoideum". In: Scientific Reports 9.1 (2019), p. 3935. DOI: https://doi.org/10.1038/s41598-019-40373-4.

(33) R. R. Aliev and V. N. Biktashev. "Dynamics of the Oscillation Phase Distribution in the BZ Reaction". In: The Journal of Physical Chemistry 98.38 (Sept. 1994), pp. 9676-9681. DoI: https ://doi .org/10.1021/ j100089a049.

(34) C. van Oss, A. V. Panfilov, P. Hogeweg, F. Siegert, and C. J. Weijer. "Spatial Pattern Formation During Aggregation of the Slime Mould Dictyostelium discoideum". In: Journal of Theoretical Biology 181.3 (1996), pp. 203 -213. ISSN: 0022-5193. DOI: https://doi.org/10.1006/jtbi. 1996.0126.

(35) V. S. Zykov and A. T. Winfree. Simulation of wave processes in excitable media. John Wiley \& Sons, Inc., 1992.

(36) E. Alvarez-Curto, K. E. Weening, and P. Schaap. "Pharmacological profiling of the Dictyostelium adenylate cyclases ACA, ACB and ACG". In: Biochemical Journal 401.1 (2007), pp. 309-316. DoI: https://doi.org/ 10.1042/BJ20060880.

(37) Y. Ohta, T. Furuta, T. Nagai, and K. Horikawa. "Red fluorescent cAMP indicator with increased affinity and expanded dynamic range". In: Scientific Reports 8.1 (2018), p. 1866. DOI: https://doi.org/10.1038/ s41598-018-20251-1.

(38) T. Gregor, K. Fujimoto, N. Masaki, and S. Sawai. "The Onset of Collective Behavior in Social Amoebae". In: Science 328.5981 (2010), pp. 10211025. DOI: https://doi.org/10.1126/science.1183415.

(39) M. Stich and A. S. Mikhailov. "Complex pacemakers and wave sinks in heterogeneous oscillatory chemical systems". In: Zeitschrift für Physikalische Chemie 216.4 (2002), p. 521. Dor: https://doi.org/10.1524/zpch. 2002.216 .4 .521$.

(40) A Gholami, O Steinbock, V Zykov, and E Bodenschatz. "Flow-driven instabilities during pattern formation of Dictyostelium discoideum". In: New Journal of Physics 17.6 (2015), p. 063007. DOI: https://doi.org/ $10.1088 / 1367-2630 / 17 / 6 / 063007$. 
(41) E. Vidal-Henriquez, V. Zykov, E. Bodenschatz, and A. Gholami. "Convective instability and boundary driven oscillations in a reaction-diffusionadvection model". In: Chaos: An Interdisciplinary Journal of Nonlinear Science 27.10 (2017), p. 103110. DOI: https://doi.org/10.1063/1. 4986153.

(42) M. Brenner and S. D. Thoms. "Caffeine blocks activation of cyclic AMP synthesis in Dictyostelium discoideum". In: Developmental Biology 101.1 (1984), pp. 136 -146. ISSN: 0012-1606. DOI: https://doi.org/10.1016/ 0012-1606 (84) 90124-6.

(43) P. Jaiswal, T. Soldati, S. Thewes, and R. Baskar. "Regulation of aggregate size and pattern by adenosine and caffeine in cellular slime molds". In: BMC Developmental Biology 12.1 (2012), p. 5. DoI: https://doi.org/ $10.1186 / 1471-213 \mathrm{X}-12-5$.

(44) O. Steinboek and S. C. Müller. "Spatial attractors in aggregation patterns of dictyostelium discoideum". In: Zeitschrift für Naturforschung C 50.3-4 (1995), pp. 275-281. DOI: https://doi.org/10.1515/znc-1995-3-417.

(45) A. T. Islam, M. Scavello, P. Lotfi, D. Daniel, P. Haldeman, and P. G. Charest. "Caffeine inhibits PI3K and mTORC2 in Dictyostelium and differentially affects multiple other cAMP chemoattractant signaling effectors". In: Molecular and cellular biochemistry (2019), pp. 1-12. DOI: https://doi.org/10.1007/s11010-019-03520-z.

(46) F. Siegert and C. Weijer. "Digital image processing of optical density wave propagation in Dictyostelium discoideum and analysis of the effects of caffeine and ammonia". In: Journal of Cell Science 93.2 (1989), pp. 325335. ISSN: 0021-9533. eprint: http://jcs . biologists .org/ content/ 93/2/325.full.pdf. URL: http://jcs.biologists.org/content/93/ $2 / 325$.

(47) K. J. Lee, E. C. Cox, and R. E. Goldstein. "Competing patterns of signaling activity in Dictyostelium discoideum". In: Physical review letters 76.7 (1996), p. 1174.

(48) M. Hendrey, K. Nam, P. Guzdar, and E. Ott. "Target waves in the complex Ginzburg-Landau equation". In: Physical Review E 62.6 (2000), p. 7627 .

(49) G. M. Whitesides, E. Ostuni, S. Takayama, X. Jiang, and D. E. Ingber. "Soft Lithography in Biology and Biochemistry". In: Annual Review of Biomedical Engineering 3.1 (2001), pp. 335-373. DOI: https://doi.org/ 10.1146/annurev bioeng.3.1.335.

(50) F. Alcantara and M. Monk. "Signal propagation during aggregation in the slime mould Dictyostelium discoideum". In: Microbiology 85.2 (1974), pp. 321-334.

(51) J. Gross, M. Peacey, and D. Trevan. "Signal emission and signal propagation during early aggregation in Dictyostelium discoideum". In: Journal of cell science 22.3 (1976), pp. 645-656.

(52) K. Tomchik and P. N. Devreotes. "Adenosine 3', 5'-monophosphate waves in Dictyostelium discoideum: a demonstration by isotope dilution-fluorography". In: Science 212.4493 (1981), pp. 443-446. 
(53) K. H. Prabhakara, A. Gholami, V. S. Zykov, and E. Bodenschatz. "Effects of developmental variability on the dynamics and self-organization of cell populations". In: New Journal of Physics 19.11 (2017), p. 113024. DOI: https://doi.org/10.1088/1367-2630/aa9391.

Acknowledgments: We are grateful to E. Bodenschatz, E. Frey, V. Zykov, O. Steinbock, F. M.-Rafiee, L. Turco, I. Guido, H. Nobach, and B. Eltzner for fruitful discussions and unknown referees for helpful comments and suggestions. Funding: T. E. acknowledges Deutsche Forschungsgemeinschaft (DFG), project Nr. GH184/1-1. E.V.H. thanks the Deutsche Akademische Austauschdienst (DAAD), Research Grants - Doctoral Programs in Germany. A.G. acknowledges the MaxSynBio Consortium which is jointly funded by the Federal Ministry of Education and Research of Germany and the Max Planck Society. Author contributions: T.E. performed experiments, E.V.H. designed and performed numerical simulations, T.E., E.V.H., A.B and A.G. analyzed data, A.B. and A.G. designed research, T.E., E.V.H., A.B., and A.G wrote the paper. Data and materials availability: All data needed to evaluate the conclusions in the paper are present in the paper and/or the Supplementary Materials. Additional data related to this paper may be requested from the authors.

\section{Supplemental material}

\section{Experimental setup}

Fig. S1 shows the PDMS mold with quadratic arrangement of the pillars, which is used in our experiments.

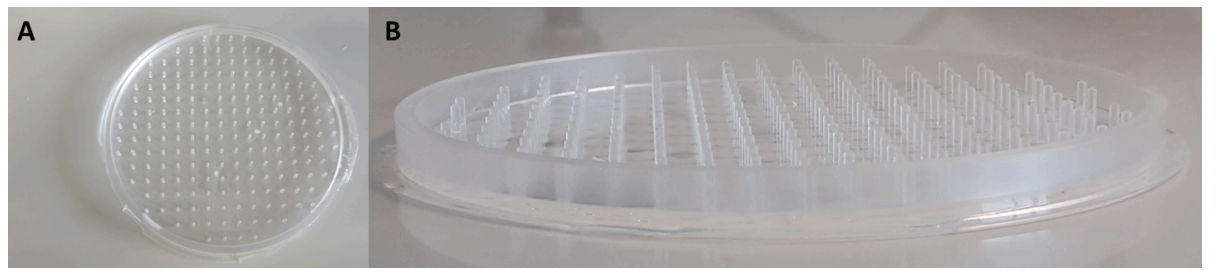

Fig. S1: Setup. A) Top view and B) side view of the PDMS block with a quadratic arrangement of the pillars that fits to a normal $100 \mathrm{~mm}$ plastic Petri dish. The pillars are $1 \mathrm{~mm}$ thick, $3 \mathrm{~mm}$ high and are spaced $5 \mathrm{~mm}$ away from each other.

\section{Spiral pinning in Dictyostelium discoideum}

Examples of spiral pinning we observed in D.d., are shown in Fig. S2. Note that spiral pinning is quite rare since the spiral cores in D.d. do not move, meaning that spirals only pin if they are initiated very close to an obstacle by chance (see Video 1). We also emphasize that the emergence of spiral or target patterns highly depends on the initial starvation time (53). In our experiments without 
4h initial cell starvation under continuous agitation, mostly spiral patterns form, independent of the underlining obstacle pattern (see Video 31).
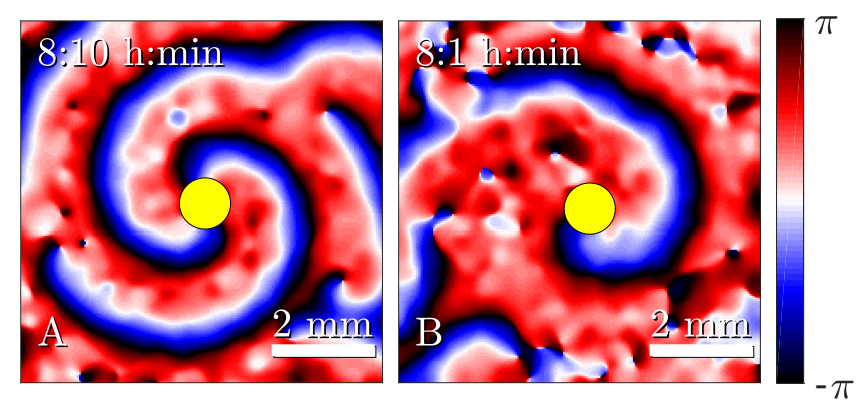

Fig. S2: Spiral pinning. Snapshots of pinned single and double arm spirals in two different experiments in the presence of $2 \mathrm{mM}$ and $0.9 \mathrm{mM}$ caffeine, respectively. Double arm spirals are rarely observed and are not stable without an anchoring pillar in our experiments (see Video 1).

\section{Experimental results in the absence of caffeine or in the absence of pillars}

Fig. S3A-B) shows an experiment without caffeine. Note that the cells show spirals which are not centered at the obstacles. Therefore, the aggregation domains are not centered on the obstacles. To repeat the exact experimental conditions similar to our pillar experiments, we performed an experiment in the presence of $2 \mathrm{mM}$ caffeine and 4 hours initial starvation but no pillars. The result of this experiment with PDMS substrate is shown in Fig. S3C-D).

\section{Frequency of firing centers}

We systematically measured the frequency of the off-pillar firing centers and compared them with the frequency of the waves initiated from the pillars. In Fig. S4, lines a, c and d are drawn to measure the frequency of the waves emitted from the pillars which are then compared with the frequency of off-pillar firing centers (lines b and e). These measurements confirm that waves started from the pillars have a slightly higher frequency than the off-pillar firing centers (see caption of Fig. S4 for the measured values).

We also performed a separate experiment to confirm that higher density clusters emit waves with higher frequencies and thereby dominate lower frequency firing centers with lower local cell density. These results are summarized in Fig. S5. The spots with higher cell density are labeled with numbers 1,3,5,7 and the period of waves emitted from these clusters are compared with the period of other centers with lower cell density (points $2,4,6,8$ ). The corresponding kymographs show clearly that higher density clusters have higher frequencies and annihilation points gradually move towards the firing centers with lower frequency and ultimately the firing center is suppressed (see Video 11). 

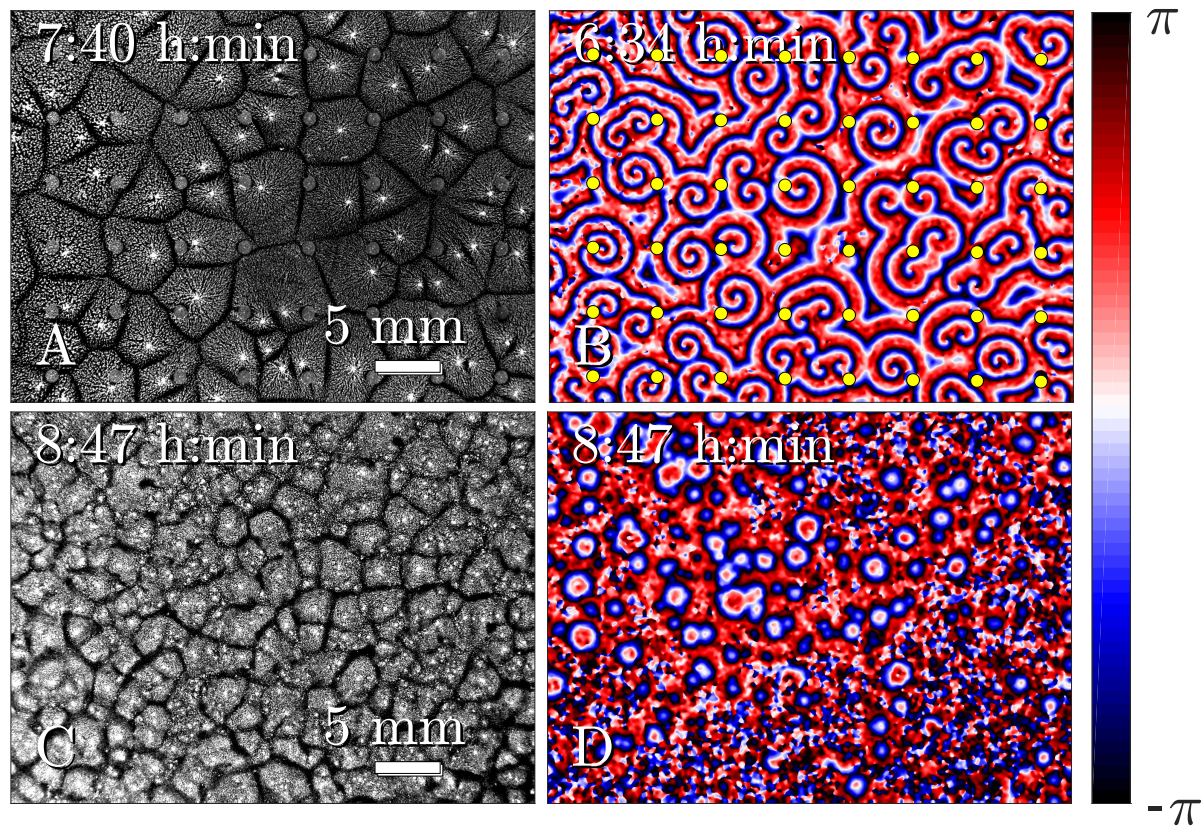

Fig. S3: Effect of Caffeine and pillars. A) Aggregation domains and B) phase map of cAMP waves in an experiment with pillars but in the absence of caffeine. C) Aggregation domains and D) phase map of cAMP waves in an experiment without pillars in the presence of $2 \mathrm{mM}$ caffeine. Note that mostly target patterns are observed. To keep the conditions similar to the pillar experiments, the cells are initially 4 hours starved in a shaking suspension and the substrate is PDMS (see also Video 3).

\section{Degree of synchronization}

To quantify the synchronization level of the waves initiated from the pillars, we made a systematic analysis of Video 5 , which is summarized in supplemental Fig. S7 and Video 7. In this analysis, all the pillars are included: around each pillar of radius $R_{1}=0.5 \mathrm{~mm}$, a larger circle of radius $R_{2}=0.75 \mathrm{~mm}$ is considered and light intensity is averaged over a ring of thickness $R_{2}-R_{1}=0.25$ $\mathrm{mm}$. We then plot for each pillar the mean intensity versus time, which is shown for two exemplary pillars in Fig. S7K-L. Each plot shows multiple minimums indicating wave initiation activities. For each activity (marked with 1 to 9 in Fig. S7J), the pillar which shows the minimum at earliest time is considered as the leading pillar. Note that at various wave initiation activities, the leading pillar is changing over time. The time points at which leader pillars start their activity (emit a wave) are shown by red dashed lines in Fig. S7J-L. We then measure the time delays of wave initiation events of the other pillars with respect to the leading pillar. This means that for each activity, we can make a histogram of the time delays. The leader pillar that initiates a wave first is presented at $t=0$, and the number of pillars that emit a wave at various delay times (with respect to the leader pillar) are presented at each histogram. For example, histogram A shows that majority of the pillars start to emit waves 20-140 sec after the leading pillar has emitted a wave at $t=0$ and only one pillar starts 


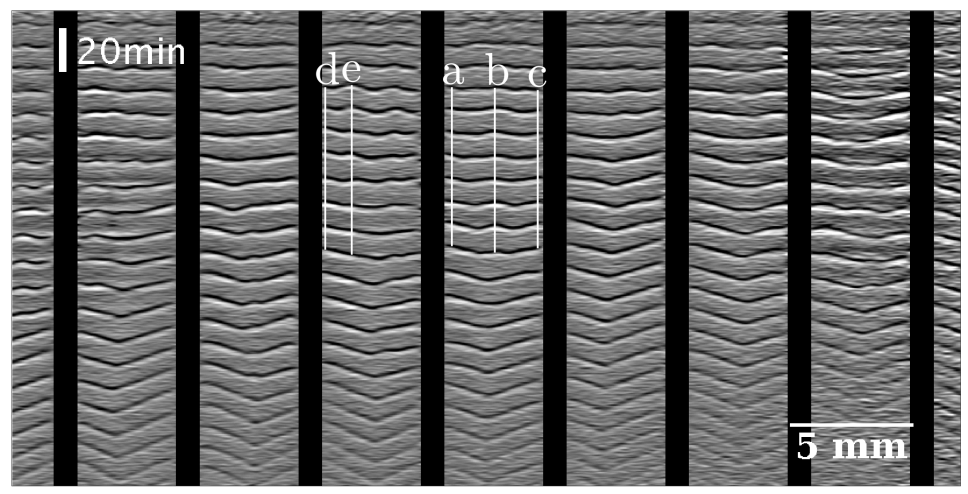

Fig. S4: Frequency of firing centers. A systematic measurement shows that waves originated from the obstacles have a slightly higher frequency (onpillar lines a and c or line d) and therefore dominate the other off-pillar firing centers (line $\mathrm{b}$ or e). The wave periods $T_{p}^{a}, T_{p}^{b}, T_{p}^{c}, T_{p}^{d}$ and $T_{p}^{e}$ measured in min along the lines a, b, c, d and e, respectively are: $T_{p}^{a}=10.57<T_{p}^{b}=10.95$, $T_{p}^{c}=10.52<T_{p}^{b}=10.95$ and $T_{p}^{d}=10.81<T_{p}^{e}=11.29$.
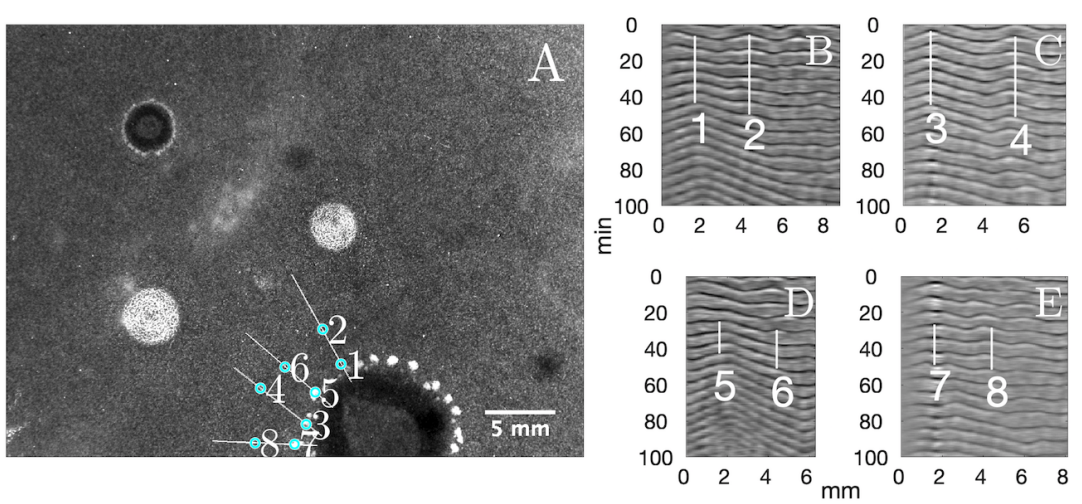

Fig. S5: Dependency of frequency on local cell density. An independent experiment to show the dependency of wave frequency on local cell density and dominance of higher frequency firing centers. The wave period $T_{p}$ measured (in $\min$ ) on the selected points are as following: $T_{p}^{1}=6.17<T_{p}^{2}=7.33$, $T_{p}^{3}=6.78<T_{p}^{4}=7.39, T_{p}^{5}=5.89<T_{p}^{6}=7.11$ and $T_{p}^{7}=7.44<T_{p}^{8}=7.78$. Note that at positions $1,3,5,7$ with higher cell density, the period (frequency) is smaller (larger). Kymographs B-E show that higher frequency wave centers 1, $3,5,7$ suppress the lower frequency firing centers $2,4,6,8$ (see Video 11).

its activity with a long delay of $200 \mathrm{sec}$. This histogram also shows that 17 pillars initiate a wave simultaneously with a time delay of $40 \mathrm{sec}$ measured with respect to the leading pillar at $t=0$; see also Video 7 . 

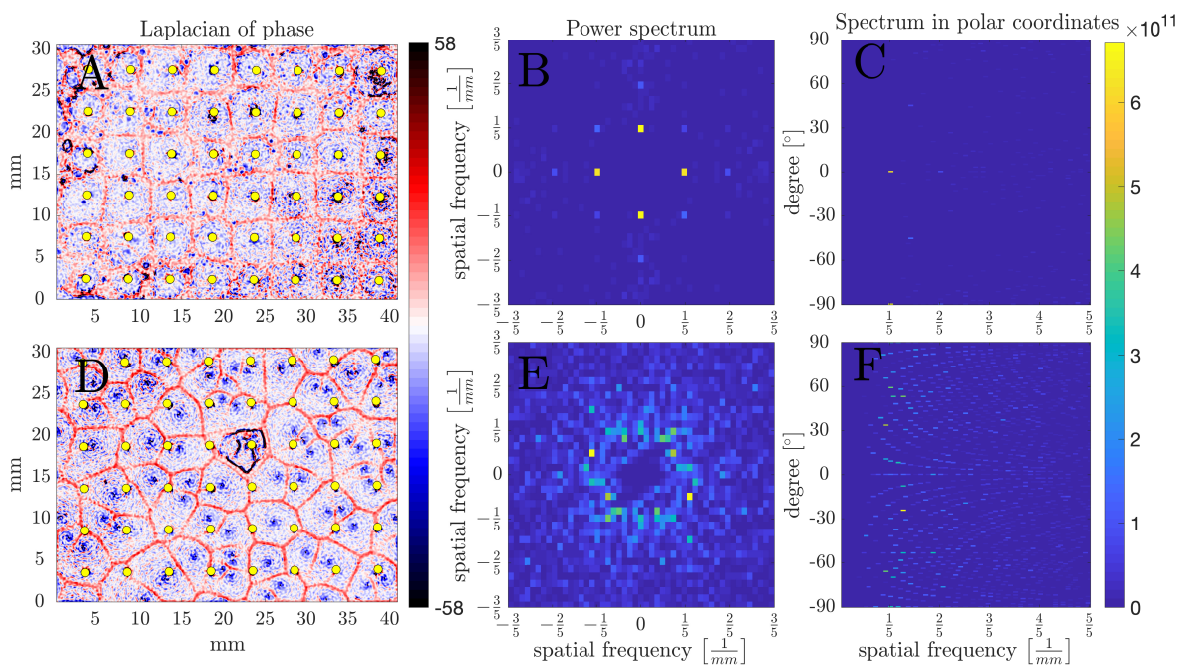

Fig. S6: Comparison of experiments with and without caffeine in the Fourier domain. 2D Fourier transform of Laplacian of phase maps in two different experiments in the presence of obstacles: A) an experiment with $2 \mathrm{mM}$ caffeine in which regular domains form and D) an experiment without caffeine forming irregular domains. Power spectrums are shown in both Cartesian (B, E) as well as in polar coordinates $(\mathrm{C}, \mathrm{F})$. While in the presence of caffeine we obtain clear peaks corresponding to $5 \mathrm{~mm}$ lattice size and $\pi / 2$-periodicity of the pattern, in the absence of caffeine, we observe two pronounced maxima at an angle of roughly $\pi / 6=30^{\circ}$ and unaligned with the pillars, which is due to randomly formed locally quasi-regular pattern.

\section{Drift velocity of the annihilation point}

We draw vertical lines starting at the annihilation point $B$ until the intersection with the wave $D$, and similarly we draw a line up from the later annihilation point $C$ up until the intersection $A$. The periods of the waves initiating from left and right pillars are $T_{1}$ and $T_{2}$, respectively (see Fig. S8). Since $\overline{A C}$ and $\overline{B D}$ are parallel, the quadrilateral $A B C D$ is a scalene trapezoid with bases $\overline{A C}=T_{1}$ and $\overline{B D}=T_{2}$. The basal angles correspond with the wave propagation velocities such that $\angle B A C=\alpha$ and $\angle A C D=\beta$ with $\tan \alpha=v_{1}, \tan \beta=v_{2}$.

Using the law of sines we calculate the other two sides of the trapezoid and arrive at

$$
\overline{A B}=\left(T_{1}-T_{2}\right) \frac{\sin \beta}{\sin (\alpha+\beta)}, \quad \overline{C D}=\left(T_{1}-T_{2}\right) \frac{\sin \alpha}{\sin (\alpha+\beta)} .
$$

We now look at the angle $\angle C B D=\gamma$ such that $\tan \gamma=v_{d}$ is the drift velocity of the annihilation point. Applying law of sines to the triangle $\triangle C B D$ we get

$$
\frac{\sin (\angle B C D)}{\overline{B D}}=\frac{\sin \gamma}{\overline{C D}}
$$



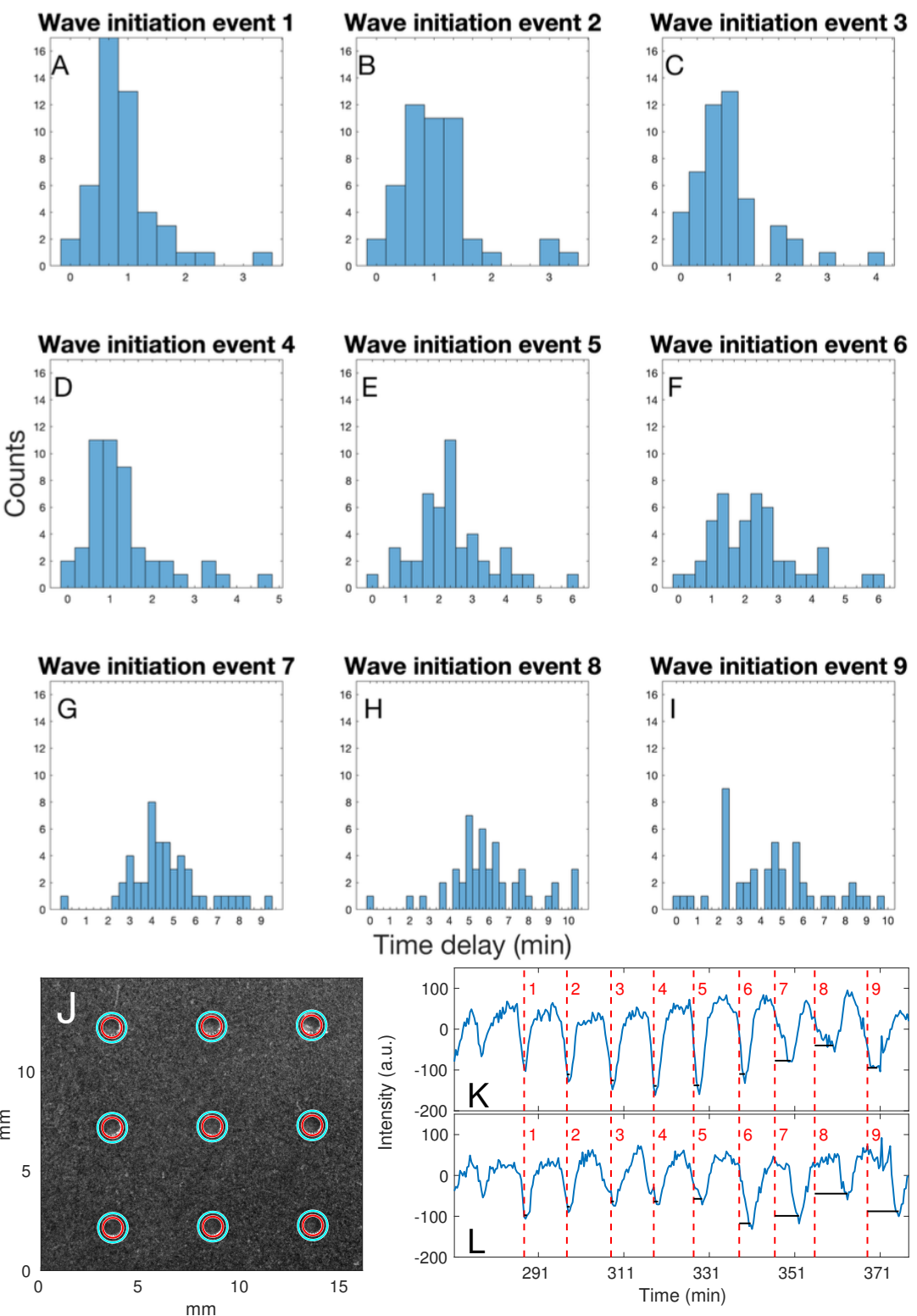

Fig. S7: Analysis to quantify degree of synchronization in in Video 7. (A-I) Wave initiation events are characterized by histograms to show the distribution of the time delays with respect to the leading pillar. Note that for example in histograms $\mathrm{B}$ and $\mathrm{D}$, three pillars simultaneously initiate a wave at $t=0$. J-L) Around each pillar of radius $R_{1}=0.5 \mathrm{~mm}$ a circle of larger radius $R_{2}=0.75 \mathrm{~mm}$ is considered and the light intensity is averaged over the area of the ring with thickness $R_{2}-R_{1}=0.25 \mathrm{~mm}$. Exemplary oscillations plotted for two arbitrary pillars are shown in $\mathrm{K}$ and L. Wave initiation events 1 to 9 by the leader pillars are shown with red dashed lines and the black lines show the delay times measured with respect to the minimums of the signal. 

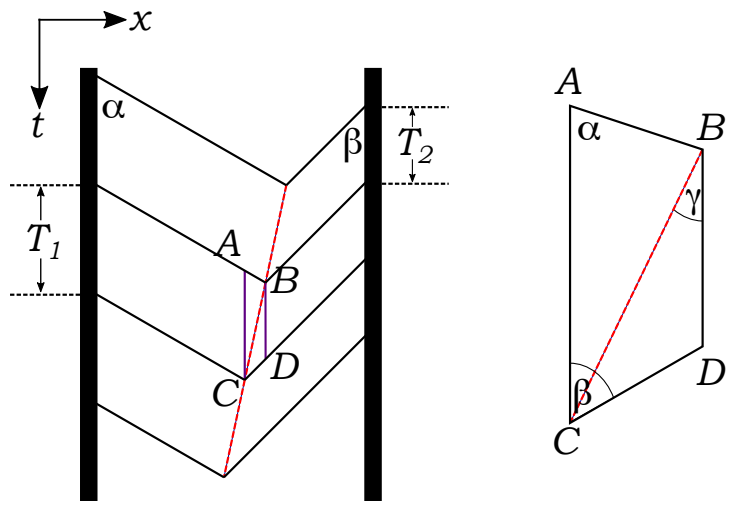

Fig. S8: Drift of annihilation point. Schematic representation in a spacetime plot of two waves coming from pillars. Thick lines represent pillars and the red dashed line marks the position of the annihilation point. Waves are emitted from the left pillar with a period $T_{1}$ and with $T_{2}$ from the right pillar. These values correspond to the vertical spacing between waves in this representation.

Substituting with the values already calculated we get

$$
\frac{\sin (\beta-\gamma)}{T_{2}}=\frac{\sin \gamma}{\left(T_{1}-T_{2}\right)} \cdot \frac{\sin (\alpha+\beta)}{\sin \alpha},
$$

expanding and simplifying we obtain

$$
\left(T_{1}-T_{2}\right)\left(\frac{1}{v_{d}}-\frac{1}{v_{2}}\right)=T_{2}\left(\frac{1}{v_{1}}+\frac{1}{v_{2}}\right),
$$

finally we arrive to

$$
v_{d}=\frac{T_{1}-T_{2}}{T_{1} / v_{2}+T_{2} / v_{1}},
$$

which in the limit case of $v_{1}=v_{2}=v_{w}$ reduces to

$$
v_{d}=v_{w} \frac{T_{1}-T_{2}}{T_{1}+T_{2}}
$$

\section{Numerical simulations without caffeine in the presence and absence of pillars}

Simulations in the absence of caffeine with and without the obstacles are shown in Fig. S11 A-D. Note that the target centers are located randomly.

\section{Numerical simulations with and without caffeine in the ab- sence of pillars}

Furthermore, we performed numerical simulations adding caffeine (larger $K_{m}$ value) but without pillars. As shown in Fig. S12, the number of wave centers is reduced and thereby the streaming domains are larger. 

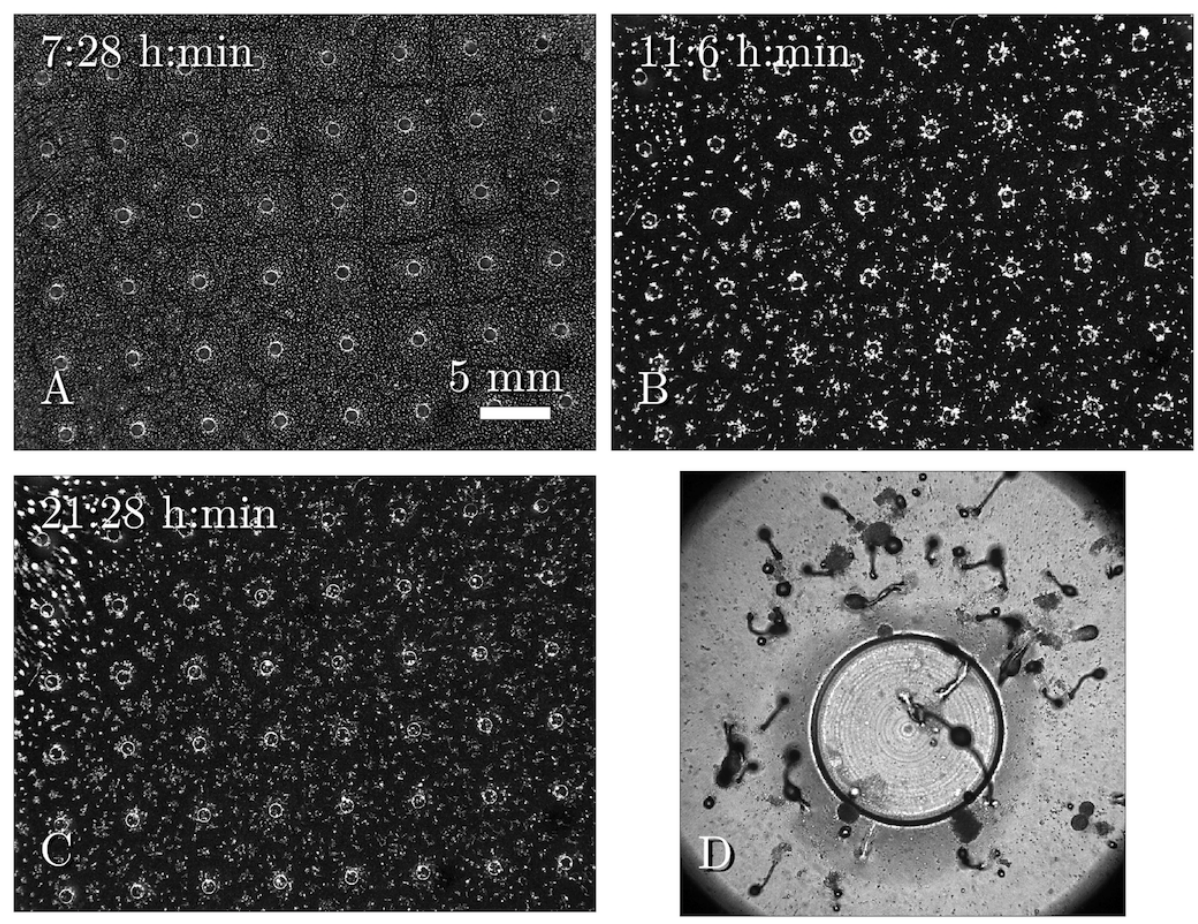

Fig. S9: Formation of fruiting bodies with a thin layer of fluid. An experiment with a thin layer of fluid (400 $\mu \mathrm{m}$ depth) and pillars of diameter 1 $\mathrm{mm}$ and height $50 \mu \mathrm{m}$ shows A) formation of regular streaming domains, B-C) aggregation of cells on the pillars and formation of fruiting bodies. A snapshot of the fruiting bodies formed on the pillar and on the area in the vicinity of the pillar is shown in part D. See also supplemental Video 12.

\section{Numerical simulations with $\epsilon$ as the control parameter}

In D. discoideum, activation of ACA is mediated by cAMP receptor, cAR1, that interacts with a G-protein, G2. It is believed that G2 $\beta \gamma$-subunit activates a phospholipid (called inositol kinase) that generates membrane-binding sites for the CRAC, which activates ACA upon recruitment to the plasma membrane (36). It is suggested that in the presence of caffeine the activation of G2 protein is reduced, thus reducing the number of ACAs in the active form. Thereby, we repeated our numerical simulations with $\epsilon$ (instead of $K_{m}$ ) as the control parameter. In the MG model, $\epsilon$ controls the ratio of the active to inactive forms of ACA: higher values of $\epsilon$ correspond to lower caffeine concentrations. As shown in Fig. S13, our results are similar to those obtained with $K_{m}$ as the control parameter.

\section{Period measurements: Experiments and Simulations}

We measured the wave periods in our simulations as a function of $K_{m}$. Higher $K_{m}$ values correspond to higher caffeine concentrations. Consistent with ex- 

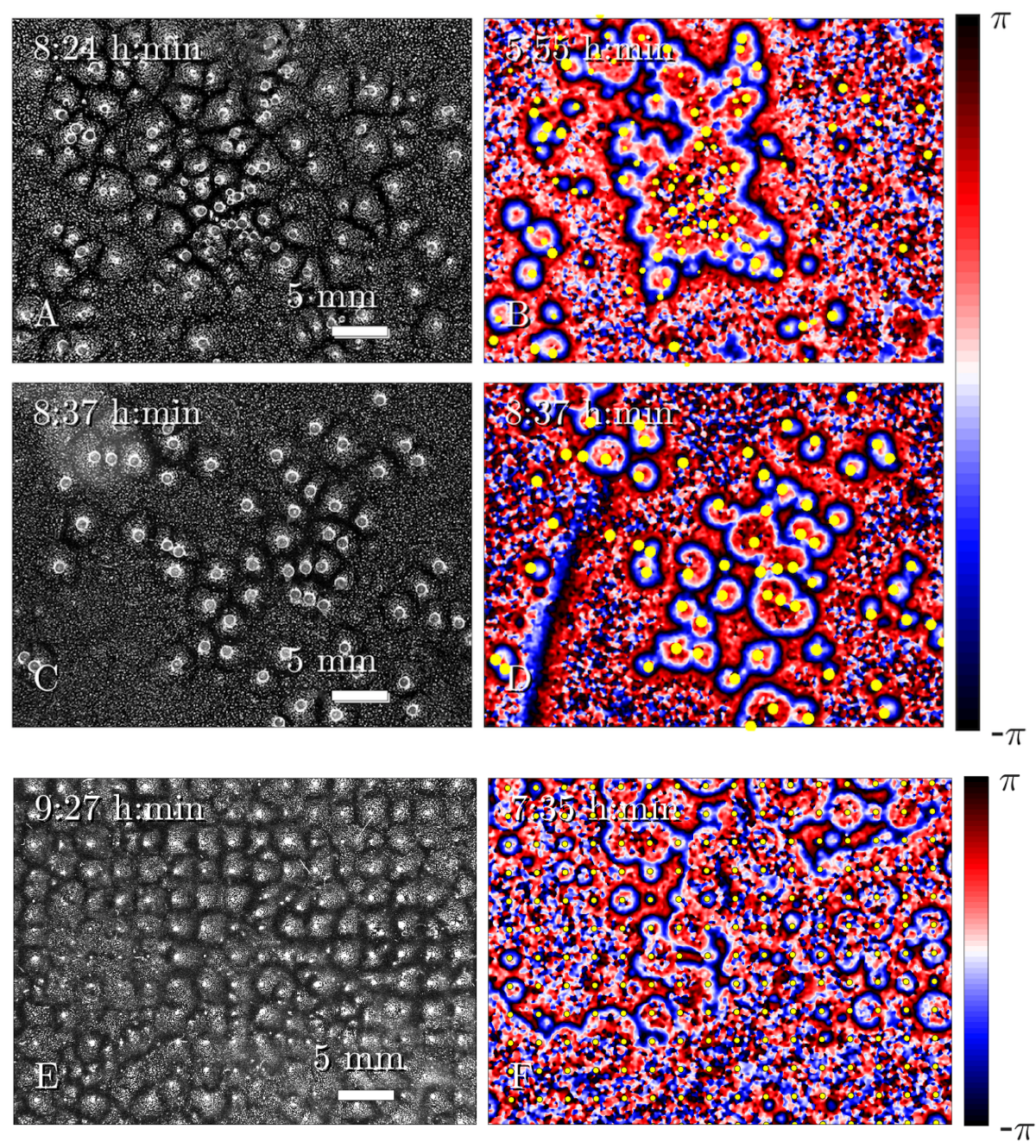

Fig. S10: Experiments with pillars of random diameter and spacing. Three different experiments with (A-B) random pillar diameter $(0.1 \mathrm{~mm} \leq$ $d \leq 1 \mathrm{~mm}$ ) as well as random center-to-center distribution, (C-D) random distribution of pillars with $1 \mathrm{~mm}$ diameter and (E-F) regular distribution of pillars of diameter $100 \mu \mathrm{m}$ and height $300 \mu \mathrm{m}$. Most of the pillars become aggregation centers. Note that pillars of diameter $100 \mu \mathrm{m}$ are spaced $2.5 \mathrm{~mm}$ away from each other and the yellow circles in part $\mathrm{F}$ are drawn three times larger than the actual size for better visibility. See also Video 13, Video 14 and Video 15. 

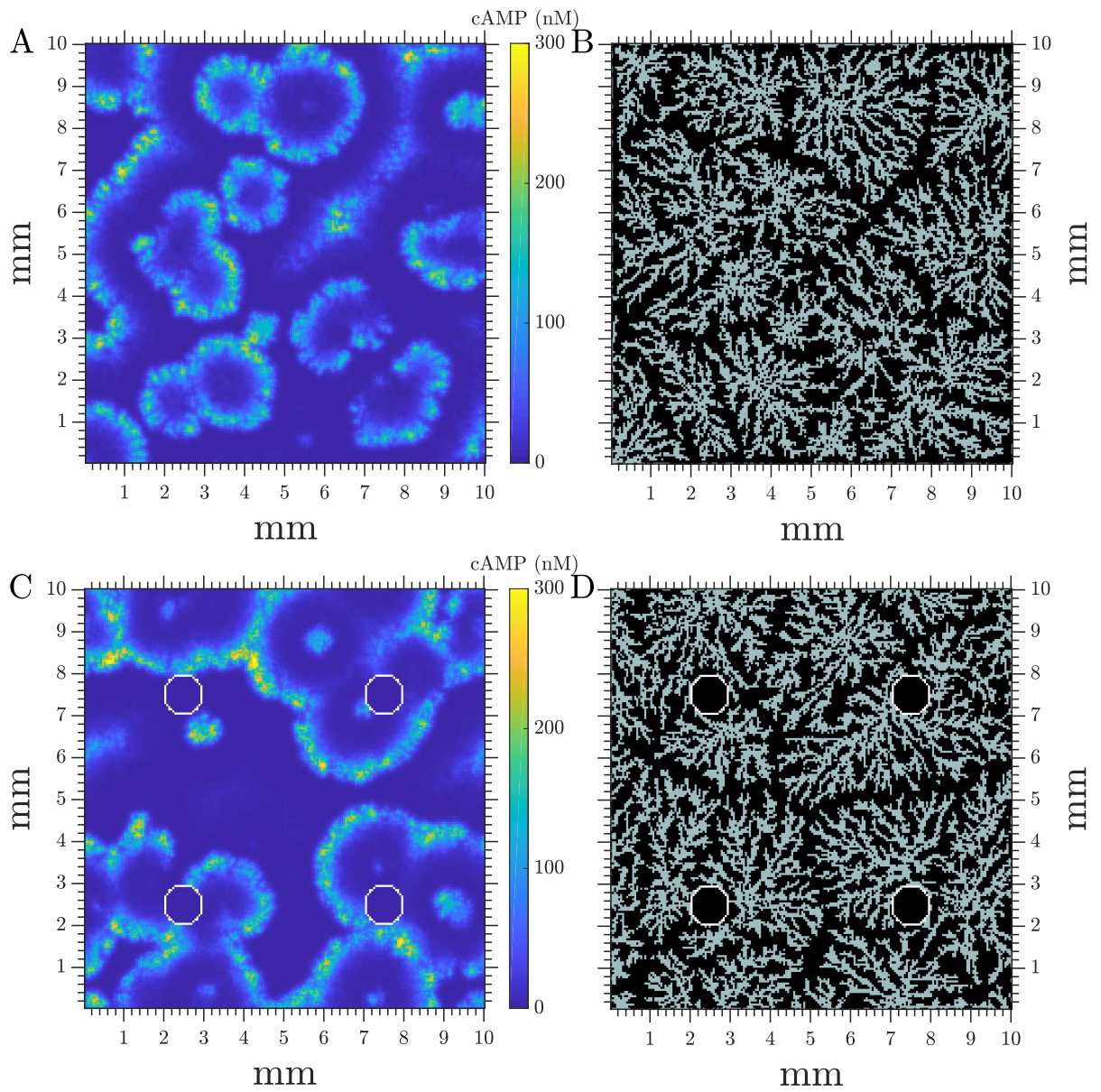

Fig. S11: Numerical simulations of cAMP waves with and without pillars and no caffeine, $K_{m}=0.40 \mathrm{mM}, k_{e}=5.5 \mathrm{~min}^{-1}, 40 \%$ of cell surface coverage, no flux boundary condition at the pillars boundaries. A) Waves of cAMP at $t=68$ min. B) Cell distribution after $t=150 \mathrm{~min}$. Grey squares show grid points containing a cell, black squares show the empty ones. C) Waves of cAMP at $t=49 \mathrm{~min}$. D) Cell distribution after $t=150 \mathrm{~min}$. See also Video 16 and Video 17. 

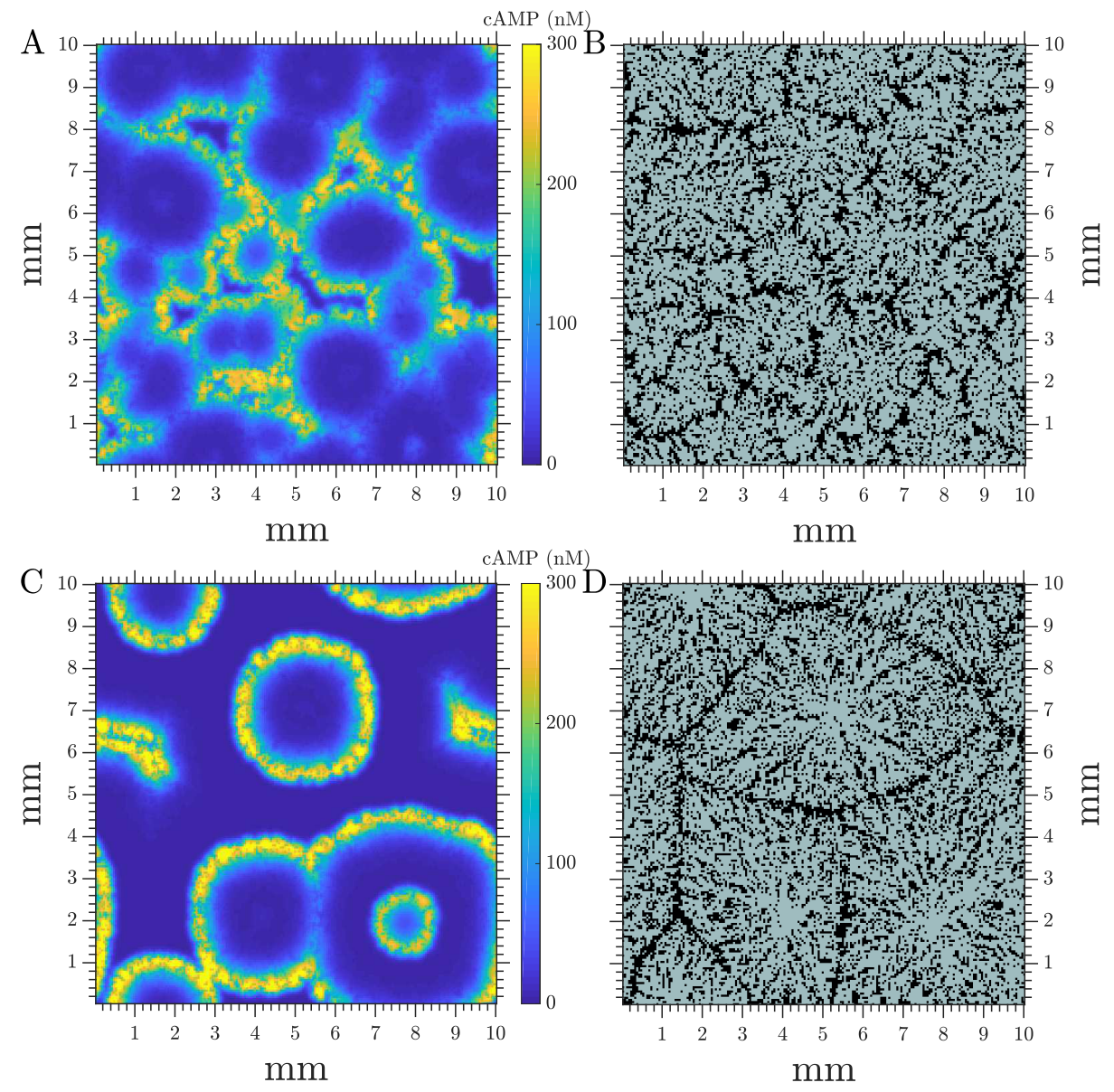

Fig. S12: Numerical simulations showing the effects in amount of target centers when adding caffeine to D. discoideum. A) cAMP waves without caffeine at $t=68 \mathrm{~min}, K_{m}=0.4 \mathrm{mM}$. B) Cell distribution after $t=100 \mathrm{~min}, K_{m}=0.4$ mM. C) cAMP waves with caffeine at $t=68 \mathrm{~min}, K_{m}=0.48 \mathrm{mM}$. D) Cell distribution after $t=100 \mathrm{~min}, K_{m}=0.48 \mathrm{mM} . k_{e}=6.0 \mathrm{~min}^{-1}, 70 \%$ of cell coverage (see also Video 19). 

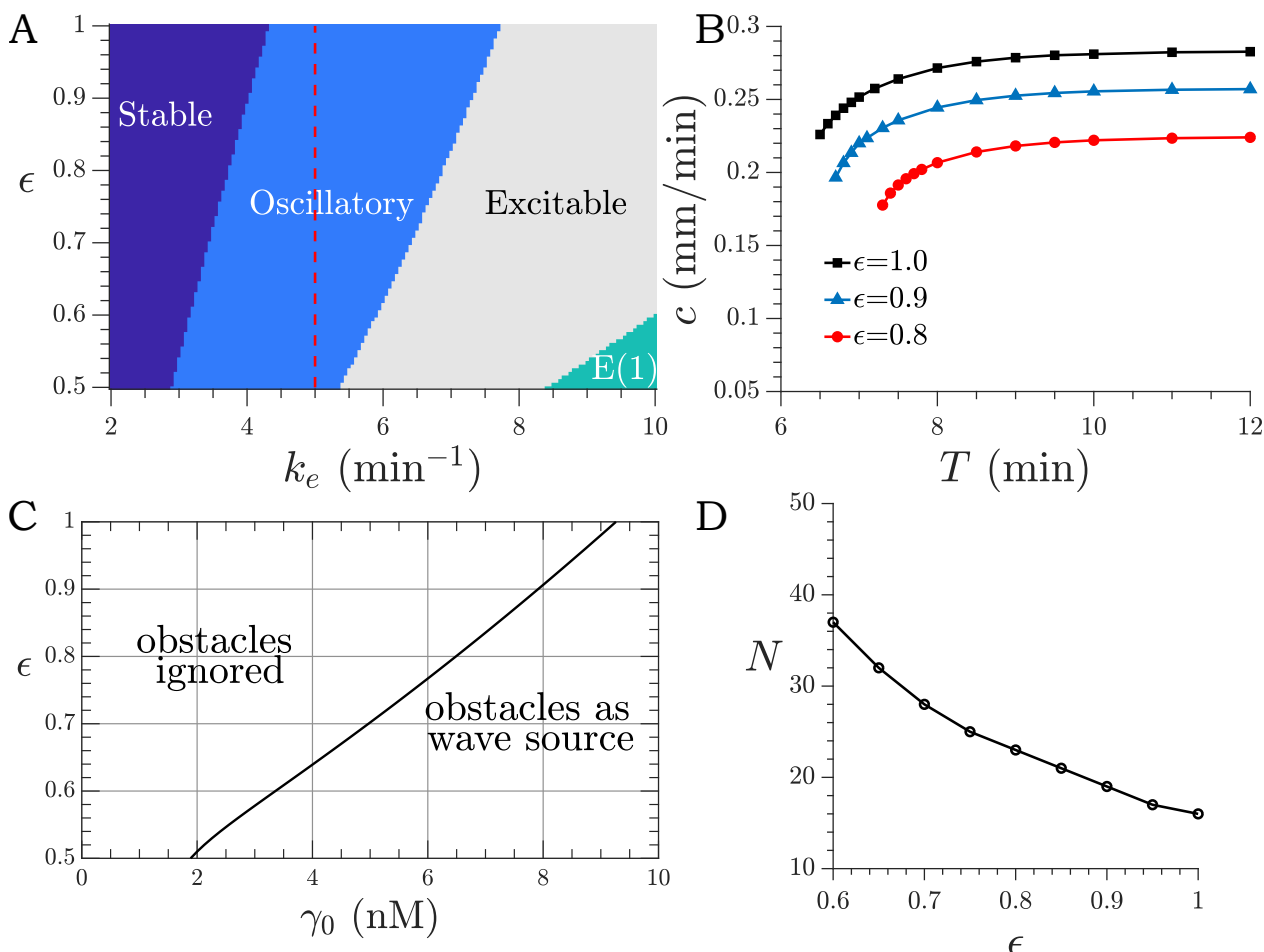

Fig. S13: A) Phase diagram of the reaction-diffusion model used for simulating the experimental setup. On the area marked as Stable, the system has one solution, which is stable. In the Oscillatory region, the system shows one unstable steady state surrounded by a limit circle. In the Excitable regime, the system has 3 steady states, two unstable ones and a stable one, which is excitable. In the regime marked as $\mathrm{E}(1)$ the system shows one steady state, which is excitable. Stability calculated through linear analysis, and excitability through no-space simulations. Red dashed line shows the path that $\epsilon$ is changed in parts $\mathbf{B}, \mathbf{C}$, and $\mathbf{D}$ at fixed value of $k_{e}=5.0 \mathrm{~min}^{-1}$. Note that higher $\epsilon$ values correspond to lower caffeine concentration. B) Dispersion relations of the supported wave trains for $40 \%$ surface coverage, where $T$ is wave period and $c$ wave velocity. Waves with periods below those shown, do not get relayed by the system. C) Effect of a boundary condition $\gamma_{0}$ : for smaller values of $\epsilon$ the minimum amount of $\gamma_{0}$ required to produce a target center decreases. D) Minimum number $N$ of consecutive cells (in 1-D) needed to produce a cluster with self-sustained oscillations. 

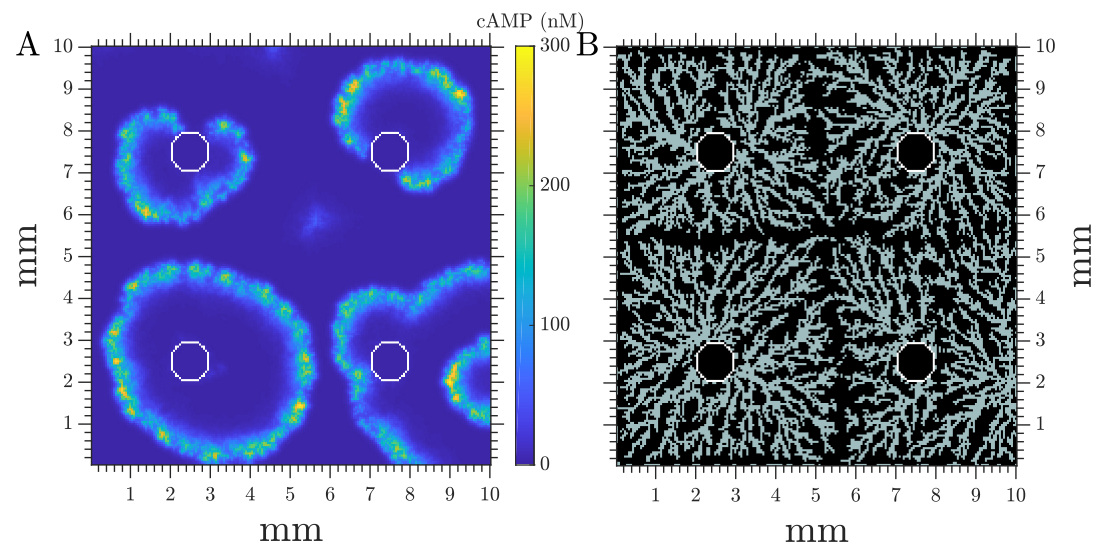

Fig. S14: Numerical simulations of cAMP waves with four pillars as obstacles with fixed boundary condition $\gamma_{0}=8 \mathrm{nM}, \epsilon=0.78$ (lower $\epsilon$ value to include the effect of caffeine), $k_{e}=5.0 \mathrm{~min}^{-1}$, and $40 \%$ of cell surface coverage. A) Concentric waves of cAMP coming out of the pillars at $t=32 \mathrm{~min}$. B) Cell distribution after $t=150 \mathrm{~min}$, showing regular domains around the pillars. Grey squares show grid points containing a cell, black squares show the empty ones (see Video 23).

periments, we observed an increase in the wave period at higher caffeine concentrations (see Fig. S15A). We also repeated the measurements for various $\epsilon$ values. Note that higher $\epsilon$ correspond to lower caffeine concentration (see Fig. S15B). Fig. S15C shows experimental measurements of the wave periods as time evolves for two cases: i) without caffeine and ii) in the presence of $2 \mathrm{mM}$ caffeine.
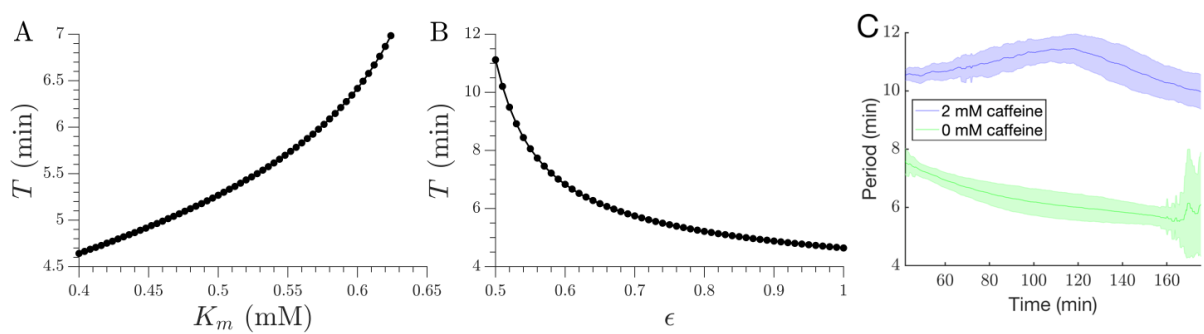

Fig. S15: Period measurements in the simulations: A) The wave period increases at higher $K_{m}$ values (higher caffeine concentrations). B) The wave period decreases at higher $\epsilon$ values (lower caffeine concentrations). C) Experimental values for period of cAMP waves as time evolves without and with 2 $\mathrm{mM}$ caffeine. For each experiment the analysis is performed on 35 aggregation centers. The signals of these centers are evaluated as a function of time and then for each time point the average and standard deviation of all time points are plotted. 


\section{Experiments with and without caffeine in the absence of pillars}

Fig. S16 shows two experiments in the presence and absence of caffeine both without pillars but PDMS substrate. For these two experiments, the cells are not initially starved in a shaking suspension. Note that in the presence of caffeine, the number of aggregation centers is reduced. It is important to emphasize that the initial starvation time of the cells plays an important role in the pattern formation of D.d. cells, as investigated in detail by (53).
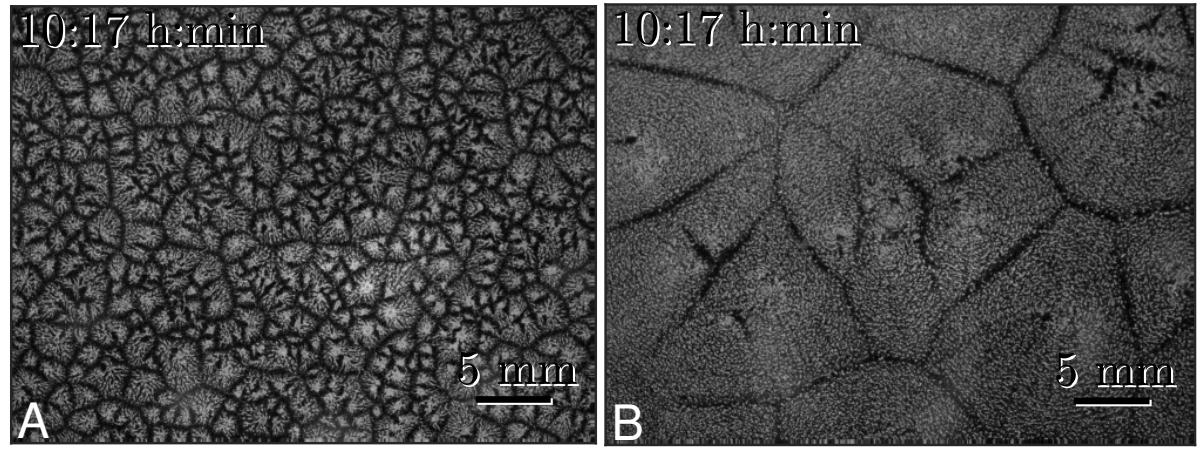

Fig. S16: Comparison of aggregation territories in two experiments: A) without caffeine and $\mathbf{B}$ ) with $2 \mathrm{mM}$ caffeine. Consistent with literature (46) and our numerical simulations, in the presence of caffeine, the number of firing centers is reduced. Note that the cells are not initially starved for these two experiments and the substrate is PDMS (see Video 30).

\section{Supplemental Videos}

Video 1. Two different experiments showing single and double arm spirals pinned to a pillar. The caffeine concentration is $2 \mathrm{mM}$ and $0.9 \mathrm{mM}$ caffeine, respectively.

Video 2. An experiment in the presence of pillars without caffeine. The left panel shows the original dark-field movie and the right panel shows the processed movie (subtracted and band-passed filtered) for better visualization of the waves.

Video 3. An experiment in the absence of pillars with $2 \mathrm{mM}$ caffeine. Please note that the substrate is PDMS. The left panel shows the original dark field video and the right panel shows the processed movie (subtracted and band-passed filtered).

Video 4. Wave propagation, streaming and formation of the regular streaming domains in an array with quadratic arrangement of pillars. Caffeine concentration is 2 $\mathrm{mM}$. 
Video 5. Images in Video 4 are subtracted every 1 min and band-passed filtered to reduce the spatial noise.

Video 6. Phase map calculated using Hilbert transform of frequency-domain filtered images of Video 5.

Video 7. A systematic analysis to quantify the degree of synchronization of the waves initiated from the pillars.

Video 8. Experiment with hexagonal arrangement of the pillars. Cells are starved initially for $4 \mathrm{~h}$ in a shaking suspension supplemented with $2 \mathrm{mM}$ caffeine.

Video 9. Experiment with triangular arrangement of the pillars. Cells are starved initially for $4 \mathrm{~h}$ in a shaking suspension supplemented with $2 \mathrm{mM}$ caffeine.

Video 10. Bright field movie of a single pillar in a quadratic arrangement. Cells are starved initially for $4 \mathrm{~h}$ in a shaking suspension supplemented with $2 \mathrm{mM}$ caffeine.

Video 11. An experiment to show that higher density cell clusters emit waves with higher frequency, thereby dominating lower frequency firing centers with lower cell density.

Video 12. Formation of fruiting bodies in an experiment with $400 \mu \mathrm{m}$ height of fluid, pillars of diameter $1 \mathrm{~mm}$ and height of $50 \mu \mathrm{m}$.

Video 13. An experiment with random diameter $(0.1 \leq d \leq 1 \mathrm{~mm})$ and random distribution of the pillars. The height of pillars is $500 \mu \mathrm{m}$.

Video 14. Pillars of diameter $1 \mathrm{~mm}$ are distributed randomly on a substrate. The height of pillars is $500 \mu \mathrm{m}$

Video 15. Experiment with pillars of diameter $100 \mu \mathrm{m}$ and height of $300 \mu \mathrm{m}$. The lattice parameter is $2.5 \mathrm{~mm}$.

Video 16. Numerical simulation showing the appearance of target centers and spirals without the addition of caffeine. Spirals marked with a white circle. $k_{e}=5.5 \mathrm{~min}^{-1}$, $K_{m}=0.4 \mathrm{mM}, 40 \%$ of cell coverage.

Video 17. Numerical simulation showing pillars being ignored without the addition of caffeine. $K_{m}=0.40 \mathrm{mM}, k_{e}=5.5 \mathrm{~min}^{-1}, 40 \%$ of cell surface coverage, no flux boundary condition at the pillars boundaries. 
Video 18. Numerical simulation showing the effects of adding caffeine to D.d.. In the presence of caffeine, the pillars act as centers even for a small accumulation of cAMP. Up: Simulation without caffeine, $K_{m}=0.4 \mathrm{mM}$. Down: Simulation with caffeine $K_{m}=0.48 \mathrm{mM} . k_{e}=6.0 \mathrm{~min}^{-1}, 70 \%$ of cell coverage, fixed boundary condition at $\gamma_{0}=6 \mathrm{nM}$.

Video 19. Numerical simulations showing the effects in amount of target centers when adding caffeine to D.d. Up: Simulation without caffeine, $K_{m}=0.4 \mathrm{mM}$. Down: Simulation with caffeine $K_{m}=0.48 \mathrm{mM}$. $k_{e}=6.0 \mathrm{~min}^{-1}, 70 \%$ of cell coverage.

Video 20. Numerical simulations showing the effect of a higher cell accumulation around pillars. $K_{m}=0.48 \mathrm{mM} . k_{e}=5.0 \mathrm{~min}^{-1}, 60 \%$ of cell coverage around the pillar, $40 \%$ in the rest of the system.

Video 21. Numerical simulation showing a triangular array of pillars, producing hexagonal patterns. $k_{e}=5.0 \mathrm{~min}^{-1}, K_{m}=0.508 \mathrm{mM}, 40 \%$ of cell coverage, fixed boundary condition at $\gamma_{0}=10 \mathrm{nM}$.

Video 22. Numerical simulation showing a hexagonal array of pillars, producing triangular patterns. $k_{e}=5.0 \mathrm{~min}^{-1}, K_{m}=0.508 \mathrm{mM}, 40 \%$ of cell coverage, fixed boundary condition at $\gamma_{0}=10 \mathrm{nM}$.

Video 23. Numerical simulations of cAMP waves with four pillars as obstacles with fixed boundary condition $\gamma_{0}=8 \mathrm{nM}, \epsilon=0.78$ (to include the effect of caffeine), $k_{e}=5.0 \mathrm{~min}^{-1}$, and $40 \%$ of cell surface coverage.

Video 24. Experiment with pillars of $1.5 \mathrm{~mm}$ in diameter. Center-to-center distance is not changed $(5 \mathrm{~mm})$. Cells are starved initially for $4 \mathrm{~h}$ under continuous agitation in the presence of $2 \mathrm{mM}$ caffeine.

Video 25. Experiment with shallow holes (100 $\mu \mathrm{m}$ deep) in PDMS. Cells are starved initially for $4 \mathrm{~h}$ under continuous agitation in the presence of $2 \mathrm{mM}$ caffeine.

Video 26. Experiment with a flat wall as obstacle. The wall thickness is $1 \mathrm{~mm}$. Cells are starved initially for $4 \mathrm{~h}$ under continuous agitation in the presence of $2 \mathrm{mM}$ caffeine.

Video 27. Concentric waves and regular streaming domains in an experiment with 50 micron high pillars. The other parameters of the array are not changed. The right panel shows the processed video. Cells are starved initially for $4 \mathrm{~h}$ under continuous agitation in the presence of $2 \mathrm{mM}$ caffeine.

Video 28. Numerical simulation showing the effect of lower degradation around pillars. $k_{e}=4.0 \mathrm{~min}^{-1}$ around pillars, $k_{e}=5.0 \mathrm{~min}^{-1}$ on the rest of the system. $K_{m}=0.48 \mathrm{mM}$. $40 \%$ of cell coverage. 
Video 29. Numerical simulation showing how pillars are ignored when there is a higher degradation rate around the pillars. $k_{e}=6.0 \mathrm{~min}^{-1}$ around pillars, $k_{e}=5.0$ $\mathrm{min}^{-1}$ on the rest of the system. $K_{m}=0.48 \mathrm{mM}$. $40 \%$ of cell coverage.

Video 30. Comparison of two experiments without (left) and with $2 \mathrm{mM}$ caffeine (right). Note that for both experiments, the cells are not initially starved in a shaking suspension and the substrate is PDMS.

Video 31. An experiment without initial cell starvation under continuous agitation shows that spiral patterns are favorable. 


\section{Discussion}

In this thesis, we study the effects of two types of perturbations on the pattern formation and aggregation behavior of $D$. discoideum, namely external flow and spatial heterogeneities. We chose these perturbation schemes because of the biological conditions the cells live in, and their properties as a reaction-diffusion-advection system. In both cases we quantified the system response and characterized the properties of the cAMP waves. Finally, we compared the experimental results to simulations of the system, testing the limits of the theoretical description and where it has to be adjusted.

\subsection{Pattern formation of $D$. discoideum under the influence of an advective flow}

D. discoideum naturally lives in the forest soil, where it is exposed to fluid flows due to rain. Experimentally, flows of up to $250 \mathrm{~mm} / \mathrm{min}$ have been measured in the soil [188]. This change in environmental conditions is quite drastic and strongly impacts the cells' behavior. The effects of a slow advective flow on the system were examined previously, both in experimental and theoretical studies $[160,189,190]$. We extend these studies by considering higher flow velocities and the effect of flow on a mutant strain that cannot form patterns normally.

\subsubsection{Fast advective flows}

Advective flows have been shown to change the waves produced by colonies of $D$. discoideum, leading to planar wave trains instead of target centers or spiral waves, which the cells produce without a flow [189]. Earlier studies were focused on the regime of low flow speeds of $V_{f}=0-5 \mathrm{~mm} / \mathrm{min}$. As flow velocities in nature can reach much higher values, we study fast flows of up to $V_{f}=50 \mathrm{~mm} / \mathrm{min}$ thereby expanding the velocity range covered by an order of magnitude. We relate our results to the effects of low flow speeds to investigate the response in pattern formation to higher flow velocities. Please note that even at these high flow velocities the shear stress in the channel is below the onset of mechanotaxis, which occurs at $0.7 \mathrm{~Pa}$ (see chapter 2, supporting material) [81].

In the passage from low to high flow speed, the shape of the waves changes from planar waves to increasingly larger and more crooked parabolic waves to finally wave stripes that fill the channel instantaneously (see chapter 2, Figs. 3 and 5). The wave shape is determined by the combination of flow and the internal cAMP dynamics. At low flow speeds $V_{f} \leq 1 \mathrm{~mm} / \mathrm{min}$, the wave shape deviates little from the planar velocity profile of the flow. For higher speeds $V_{f}>1 \mathrm{~mm} / \mathrm{min}$ the wave shape becomes more 
parabolic, which depends on the reaction-diffusion dynamics of the system. The original MG model consists of three equations, describing the external and the internal cAMP concentration as well as the fraction of active cAMP receptors on the membrane. A common simplification of this system can be done by assuming that internal cAMP is immediately transported outside of the cells, resulting in only two components. We find that the two-component version of the MG model cannot reproduce the experimentally observed wave shapes, emphasizing the importance of gradual cAMP accumulation inside the cells before its transport to the outside medium. In contrast, the full threecomponent model does create elongated parabolic wave fronts as found experimentally. This is due to the inclusion of the slow dynamics of intracellular cAMP and its gradual release to the extracellular medium. Our experiments confirm that the key assumption for the reduction to a two-component model is not valid at flow speeds $V_{f}>1 \mathrm{~mm} / \mathrm{min}$.

Furthermore, we quantify the wave characteristics, finding that while the wave speed along the channel is set by the advective flow speed, the wave speed in transversal direction does not change according to different flow speeds. Indeed, the transversal speed remains constant at about the value at which the waves move in an undisturbed system $(0.3-0.5 \mathrm{~mm} / \mathrm{min}[48,101,110,111])$. Together with the extremely elongated shape of the waves at these flow speeds, this means that we observe long stripe-like waves, which fill the channel in flow direction immediately after their creation and then slowly propagate towards the sides.

Similarly, the period of the waves found in this system is constant for the different flow speeds. Therefore the pattern formation system still fulfills its key purpose of enabling aggregation if perturbed by flow. Additionally, we find that while the cells aggregate even under fast flows, the aggregation territory shape is changed. Indeed, the cells aggregate in triangular aggregation territories, with one of the triangles vertices facing against the flow direction. This effect is probably based on the flow advecting cAMP downstream of each aggregation center and thereby suppressing signaling in the upstream direction. Similar aggregation behavior has been described before in aggregation under flow [148].

Moreover, we measure a linear scaling of the wave thickness as a function of the applied flow velocity. We believe this phenomenon is due to a combination of a constant cAMP release with time and the influence of the flow. The cAMP produced by the cells is spread over a larger area at higher flow speeds, leading to an increase in wave width. The phenomenon of wave thickness scaling with flow velocity has been reported in the FitzHugh-Nagumo model [191]. Indeed, it is a more general feature of advected excitable media, as a supra-threshold excitations will be spread over a larger area at higher flow speeds. Similar results were found in experiments and simulations exposing D. discoideum to electrical fields $[192,193]$. Changes in the waves were reported, with waves moving towards the positive electrode propagating more quickly and having a larger width [192]. The opposite was true for waves moving towards the negative electrode. The effect was included in simulations by considering additional electrically driven advection of cAMP due to its charge [193]. Therefore, applying an electrical field has a similar influence on the cells as a fluid flow does. However, with an electrical field, a considerable warming of the cells has been reported, which could lead to unintended side effects, as wave speed and period scale with it $[111,192]$. Additionally, the electrical 
field could influence the ion channels in the cell membrane, making the cell response even harder to analyze.

At high flow speeds $V_{f}>5 \mathrm{~mm} / \mathrm{min}$, the wave initiation point is crucial for the wave shape and its behavior. If waves are created close to the vertical top or the bottom of the channel, they produce the aforementioned stripe-like shape. However, waves initiated in the vertical middle of the channel develop to extremely elongated parabolic waves. The defining feature here is the difference between wave velocity along the channel $v_{\|}$and the velocity in transversal direction $v_{\perp}$. As $v_{\|}$scales with applied flow speed, but $v_{\perp}$ does not, any wave will quickly be spread out along the channel and then more slowly move in transversal direction. Additionally, waves were only created at the inlet of the channel, in contrast to low flow speed experiments, where waves are produced along the entire length of the channel. This is probably due to influences of the boundary of the system, to which we supply a fresh cAMP-free buffer. An explanation of this could be that unbounded PDE produced by the cells upstream is advected downstream, resulting in a slightly increased degradation rate downstream, which would suppress wave formation. In the three-component model with a developmental path scenario for wave initiation we find a wide variety of wave shapes quite similar to the ones observed experimentally.

Finally, we find that the waves react to a change in the applied flow speed immediately, confirming that we observe trigger and not phase waves (chapter 2, Fig. 10). Waves in the simulations behaved similarly upon flow speed changes, confirming that we chose the cell state in phase space appropriately. In some cases we also find a transient decrease in the wave period upon application of a higher flow speed (chapter 2, Fig. 13). The reason for this is probably that waves already in the channel cannot change their wavelength, as it is set at wave creation. An increase in the flow speed then leads to more waves passing through the same spot in the channel and thus a decrease of the local wave period. This phenomenon might be interesting to investigate further, as it should have natural bounds. If the flow speed is switched to a large value, some of the waves should be extinguished while moving into the refractory wake of each other. If it is switched to a small value instead, spontaneous patterns might form in the channel.

Through mapping the response of colonies of $D$. discoideum to high advective flow speeds, we validate the model we use and its assumptions. Pattern formation and aggregation is robust to high flow speeds. Especially, the period as a key parameter is independent of the applied flow speed. Moreover, we find that aggregation persists for high flow speeds. However, at velocities $V_{f}>5 \mathrm{~mm} / \mathrm{min}$ the wave shape is dependent on the wave initiation site, resulting in more complex patterns. Through comparison to simulations we find that the reduction to two components in the MG model is only valid for low flow speeds and the dynamics of the third component are needed to explain wave shapes at high flow speeds.

\subsubsection{Boundary-driven waves in pds $\mathrm{A}^{-}$cells}

This study expands on and verifies theoretical results of Vidal et al predicting boundarydriven waves in a flow-through setup of cells with a zero boundary condition upstream [172]. This prior research indicated a region of the $k_{e^{-}} \sigma$ phase space in which the system 
is convectively unstable, meaning that wave creation was possible only if a flow of an adequate magnitude was applied. Considering that the predicted convectively unstable region was found at low degradation rates $k_{e}$, we decided to use pdsA- cells. This knockout mutant of $D$. discoideum does not produce pdsA, which accounts for most of the degradation of extracellular cAMP during the aggregation stage [49]. For this reason, $\mathrm{pdsA}^{-}$cells do not show patterns without an applied flow, allowing us to investigate its impact more clearly. It has been suggested by Gregor et al that a fresh buffer flow can act as effective degradation in small cell colonies of about 200 cells, rescuing the cAMP oscillations [113, supporting material, Fig. S10].

We find that a fresh buffer flow can indeed act as a replacement for degradation, inducing wave creation at the upstream boundary. These waves then decay in intensity while moving along the channel. This effect can be explained by considering that the cAMP concentration increases along the channel, making the cells less excitable the farther down the channel they are, until they saturate and do not react to cAMP. At high flow speeds the system shows traveling waves as described in section 5.1.1. This indicates that flow can fully replace degradation in large colonies of cells, in accordance to results from an earlier study [113]. In theoretical models a destabilization of reactiondiffusion systems by a Dirichlet boundary condition has been proposed as well $[169,171]$

We do not observe waves if the applied flow is too weak $V_{f}<0.3 \mathrm{~mm} / \mathrm{min}$ and during the first three hours of flow even at higher speeds. This behavior did not change with initial starvation of the cells in shaking culture, confirming that pdsA ${ }^{-}$cells cannot start the developmental process leading to pattern formation and aggregation on their own. The first three hours with flow but without waves can then be interpreted as the initial developmental period the cells need to activate the molecular machinery necessary for cAMP production and chemotaxis.

Moreover, we find that the dynamics of the system are dependent both on the applied flow speed and on the amount of time it has been applied, often changing fundamentally during the experiment. These variations in the pattern formation result in an early and a late regime with different characteristics. During the early regime, the waves decay more quickly along the channel and pattern formation is less pronounced.

We quantify the system response for a range of different flow speeds for both early and late regimes, see (chapter 3, Fig. 2). The wave speed is set by the applied flow speed as seen in the experiments described in section 5.1.1. We measure the characteristic decay length during the early regime and find that it scales linearly with applied flow speed. This is reasonable, considering that constantly cAMP producing cells will create a gradient of increasing cAMP along the channel. At higher flow speeds the cAMP gradient is less steep and cells in a longer part of the channel are below saturation conditions, which corresponds to a longer decay length.

For flow speeds $V_{f}<0.6 \mathrm{~mm} / \mathrm{min}$, we find longer periods than the typical $6 \mathrm{~min}$ period of the waves. For a flow speed of $V_{f}=0.3 \mathrm{~mm} / \mathrm{min}$ the period is about $20 \mathrm{~min}$, which is much longer than the period observed normally. The period decreases with increasing flow speed and returns approximately to the value seen in the unperturbed system for $V_{f}=0.6 \mathrm{~mm} / \mathrm{min}$. For low flow velocities the period slightly increases in the late regime. At about $V_{f}=0.6 \mathrm{~mm} / \mathrm{min}$ the system seems to have relaxed to its 
natural frequency. We do not find a clear trend for the wavelength of the oscillations, as it differs strongly between experiments.

Comparing the experimental results to simulations, we find that the appropriate parameter range is at very low degradation rates, where the system is unstable with a Dirichlet boundary condition. We find similarly decaying waves with longer decay lengths at higher flow speeds and a minimum flow velocity for wave creation. In contrast to the experimental observations, there is a maximum flow velocity, above which no wave creation is observed. Further, the period increases with applied flow speed in contradiction to the experiments. We believe that these contradictions hint at two different wave creation mechanisms being present in the system. While boundary-driven waves can explain the waves at low flow velocities, at higher speeds the period is probably set by the internal timescale for cAMP production and receptor resensitization.

The cells aggregate in experiments with applied flow speed of $V_{f} \geq 0.6 \mathrm{~mm} / \mathrm{min}$ and cells close to the inlet start aggregating first. The length in which we find cell aggregation increases at higher flow speeds. This emerges quite clearly in experiments where we study a straight main channel that has a side channel (chapter 3, Fig. 6). The speed in the side channel is lower because of hydraulic resistance, resulting in a shorter domain of cells showing aggregation. We performed extensive bright-field microscopy to closely look at the rescue of aggregation in $\mathrm{pdsA}^{-}$cells in the presence of flow (chapter 3 Fig. 5).

Moreover, we find that the cells show spontaneous pattern formation after the flow has been turned off, if flow of $V_{f} \geq 0.6 \mathrm{~mm} / \mathrm{min}$ was applied and for at least 3.5 hours. The spontaneous waves appear after about 6 hours upstream of the flow-through channel, independently of how long flow has been applied. These waves show a much longer period compared to the natural waves, on the order of 15-25 min. This leads us to believe that a different PDE, most likely PDE4 or PDE7, is activated, if flow is applied for long enough. The flow might allow for the formation of sub-threshold cAMP pulses, which then in turn induce PDE activation [113]. The length of the channel, in which cells are reconstituted by flow, seems to increase with applied flow speed and the duration the flow was applied, confirming that the flow is the crucial element. PDE7 is expressed during the first 3 hours of development in WT cells, which is before PDE4 is expressed starting after 5 hours of development [49]. This leads us to believe that the PDE in question is PDE7. Concerning reaction dynamics there is little difference between the two PDEs. Both have an about ten times lower affinity to cAMP than PDE1 [49]. Therefore, one of the next steps in this research should be a careful biochemical analysis of pdsA- cells which are subjected to flow.

Finally, we rescue pdsA ${ }^{-}$cells by supplying them with a solution that is preconditioned by Ax2 cells in the reservoir. For vegetative Ax 2 cells this leads to fully recovered waves after an initial 3 hours period without waves. In contrast, Ax2 cells that were initially starved for 4 hours induced waves traveling through the system immediately. The decay length of these waves increased exponentially with time (chapter 3, Fig. 10). This phenomenon is probably caused by the inability for cAMP production and thus wave relay of vegetative pdsA- cells. Thus, the cAMP produce by the Ax2 cells moves through the channel whilst being degraded by the emitted pdsA. The cAMP waves traveling 
down the channel activate the $\mathrm{pdsA}^{-}$cells, allowing them to produce more cAMP and thereby increasing the length the waves can be relayed. In the simulations, an upstream boundary that shows an oscillating cAMP concentration together with a gradual increase in cAMP production throughout the channel produced similar results.

In summary, we confirm experimentally that a Dirichlet boundary condition can destabilize an otherwise stable pattern forming system, as was predicted theoretically $[169,171]$. As fluid flows are common in nature, this wave creation mechanism should be applicable in other normally stable pattern forming systems. Moreover, we gain insight into the developmental dynamics of $D$. discoideum and confirm that removal of extracellular cAMP is defining for cell development. Furthermore, we show that flow can replace degradation, which might be relevant in other systems using PDEs to allow for signal relay such as the human body where dysregulation of cAMP signaling is linked to tumor growth [194].

\subsection{Pattern formation of $D$. discoideum under the influence of non-excitable obstacles}

In their natural environment, the soil, D. discoideum amoebae produce patterns and aggregate in complex geometries. These geometrical constraints can be considered as non-excitable obstacles, which are randomly distributed in three dimensions. As a first step towards understanding this system, we study pattern formation of D. discoideum perturbed by a uniform two-dimensional grid of obstacles. Our setup consists of cylindrical PDMS obstacles arranged as a grid on a flat PDMS surface, as shown in (chapter 4, Fig. S1). The interaction of patterns in excitable media with non-excitable areas has been studied intensely in both the BZ-reaction and heart tissue with a focus on spiral pinning $[195,196]$.

We find that, under normal conditions, spirals in D. discoideum mostly ignore obstacles. If a spiral is initiated close enough to an obstacle, its tip can pin to the obstacle, sometimes leading to multi-armed spirals (chapter 4, Fig. S2). However, since spiral tips in D. discoideum do not meander, there is little interaction if the core is not close to an obstacle. Under these conditions, a wave simply breaks at the obstacle and then recombines again behind it.

We altered the excitation threshold of the cells by adding 1-5 mM caffeine to the cell solution. When we combined this with an initial starvation for 4 hours in shaking culture, we find that concentric waves appear originating from the obstacles. In this case, the pattern formation starts with bulk oscillations and then shows concentric waves. The concentric waves initiated at the walls of the obstacles have a slightly lower period than the off-center waves, leading to them dominating the system dynamics (chapter 4, Fig. S4). Together with the annihilation of cAMP waves, this leads to regular aggregation territories centered on the obstacles, as shown in (chapter 4, Fig. 1). It has been shown before that target centers are prevalent if the cells are starved for 4 hours before the experiment [176]. 
This phenomenon is quite robust as long as we alter the excitability of the cells by adding caffeine. We also observed it in different obstacle geometries, using a rectangular, triangular and hexagonal grid, see (chapter 4, Fig. 1 and 3). Further, the defining factors seem to be the influence of caffeine and spatial inhomogeneities in the system, since we also see this behavior for shallow holes (100 $\mu \mathrm{m}$ depth) and a straight wall. Moreover, we changed the shape of the obstacles and found the same behavior using triangular obstacles. Consequently, the next step in our study on this system is direct visualization and measurement of the cAMP concentration, as was proposed using fluorescent probes [166].

During aggregation the cells first form clumps off-center and then stream towards the centers (chapter 4, Fig. 4). If cell aggregation is allowed to proceed under a thin layer of liquid, the amoebae will also produce fruiting bodies at the obstacles (chapter 4, Fig. S9).

Comparing the experimental results to simulations, we find that the inclusion of the effect of caffeine by changing the Michaelis-Menten constant of the response to cAMP cannot explain the effect we observe. In contrast, we are only able to reproduce these results by assuming an additional inhomogeneity in either adsorption and thereby depletion of PDE on the PDMS or slightly increased local cAMP levels. Additionally, a locally higher cell density showed the same effect, as it led to an increase in the local wave frequency. We could not verify any of these assumptions experimentally. We did, however, find proof for the induction of a higher frequency at a spot of locally increased cell density, see (chapter 4, Fig. S5). In addition, the effect of caffeine in the simulations is clear, with an increase in the minimum amount of cells necessary to create a center (chapter 4, Fig. 5). Considering that PDMS has been shown to absorb small molecules such as cAMP, we cannot rule out an influence of the obstacle material [197]. Nonetheless, we found the same behavior in a setup of PMMA obstacles. Similarly, we found the same behavior when we increased the viscosity of the medium by adding $5 \%$ ficoll to exclude hydrodynamic interactions.

To summarize, we find a strongly different behavior in the pattern formation and aggregation system in D. discoideum if supplied with caffeine and starved. This phenomenon represents a control mechanism on pattern formation in the amoebae. In other excitable systems, such as the BZ-reaction, possible ways of taking control over the pattern formation have been demonstrated, by using a light sensitive variant of the reaction and by application of an electric field $[198,199]$. In $D$. discoideum applying an electric field has been shown to control pattern formation to a certain degree, leading to an increase or decrease in wave speed and thickness depending on wave direction relative to the polarity of the field $[192,193]$. We find a new control mechanism for pattern formation in $D$. discoideum that could be used to further elucidate its pattern formation dynamics. 



\section{Conclusions and Outlook}

In this thesis, D. discoideum was experimentally studied as a pattern forming system while mimicking its natural environment. We explored the properties of this excitable system in a number of ways, testing the limits of the underlying theoretical descriptions. Additionally, our studies probed the influences of environmental conditions that the amoebae are exposed to in the soil. These are fluid flows induced by rain and spatial heterogeneities, which act on the cells' pattern formation and aggregation.

We investigated the extension of the reaction-diffusion system by considering an advective flow. This perturbation of the reaction-diffusion system is theoretically interesting in its impact on pattern formation. Additionally, it is relevant biologically, tackling the question of whether the cells can aggregate under the influence of flows induced by rain. We found implications of a possible wave creation mechanism and on the validity of a frequently used simplification in the model. Furthermore, we found that at high flow speeds wave initiation is suppressed everywhere in the channel except for at the upstream boundary. Additionally, more complex wave patterns are found than at low flow speeds.

Moreover, we studied the interplay of a central mutation in the cells and its apparent alleviation by flow. From a theoretical standpoint we verified that a Dirichlet boundary condition can destabilize a reaction-diffusion-advection system. Experimentally, we gained insight into the cell development and possible homologous enzymes.

Finally, we studied the influence of an inhomogeneous geometry on the cells. The results correspond to establishing a control over pattern formation in this system. On the theoretical side, we found a mechanism for inducing target centers and could explain the decrease of the number of wave centers under the addition of caffeine.

Since $D$. discoideum colonies form an excitable medium, we believe that the theoretical insights gained through experimental studies can be transferred to other systems. Reaction-diffusion-advection systems are quite widespread in nature and especially the destabilization through a Dirichlet boundary should be relevant to the study of other systems such as the BZ reaction.

As colonies of $D$. discoideum are a complex pattern forming system, they can be manipulated in a number of ways. This should allow for many more studies altering the pattern formation in subtle ways. A prime example for this approach would be inducing spatial variability in the system parameters [200]. This could be done via an uneven application of a drug or an inhomogeneous system temperature that locally changes reaction rates [111,201]. Another approach would be to create spatial inhomogeneities by depositing cells with different properties or cells at varying densities [176]. Moreover, the cells could be manipulated with ultrasound either directly or indirectly by inducing a localized flow via ultrasonic streaming [202-204]. Additionally, spiral pinning would 
Chapter 6 - Conclusions and Outlook

be an interesting topic to explore, using non-excitable obstacles and possibly a flow or an electric field to induce spiral core movement $[192,195,199]$. In the context of our studies, it would be beneficial to visualize cAMP with directly specific fluorescent probes, as was shown recently [166]. The tsunami $D$. discoideum strain could be used to study long term pattern formation, as the cell streaming starts much later in this mutant [205]. In such a system one could study the response to repeated or very subtle perturbations. 


\section{Bibliography}

[1] M. C. Cross and P. C. Hohenberg. Pattern formation outside of equilibrium. Rev. Mod. Phys., 65:851-1112, Jul 1993.

[2] Marc Lämmel, Anne Meiwald, Hezi Yizhaq, Haim Tsoar, Itzhak Katra, and Klaus Kroy. Aeolian sand sorting and megaripple formation. Nature Physics, 14(7):759$765,2018$.

[3] Jana Lasser. Geophysical pattern formation of salt playa. PhD thesis, GeorgAugust-Universität, Göttingen, 2019. http://hdl.handle.net/21.11116/0000-00033962-F.

[4] Geoffrey Ingram Taylor. Viii. stability of a viscous liquid contained between two rotating cylinders. Philosophical Transactions of the Royal Society of London. Series A, Containing Papers of a Mathematical or Physical Character, 223(605615):289-343, 1923.

[5] Alan Mathison Turing. The chemical basis of morphogenesis. Philosophical Transactions of the Royal Society of London. Series B, Biological Sciences, 237(641):37$72,1952$.

[6] Akiko Nakamasu, Go Takahashi, Akio Kanbe, and Shigeru Kondo. Interactions between zebrafish pigment cells responsible for the generation of turing patterns. Proceedings of the National Academy of Sciences, 106(21):8429-8434, 2009.

[7] Han-Sung Jung, Philippa H Francis-West, Randall B Widelitz, Ting-Xin Jiang, Sheree Ting-Berreth, Cheryll Tickle, Lewis Wolpert, and Cheng-Ming Chuong. Local inhibitory action of bmps and their relationships with activators in feather formation: Implications for periodic patterning. Developmental Biology, 196(1):11 $-23,1998$.

[8] Stefanie Sick, Stefan Reinker, Jens Timmer, and Thomas Schlake. Wnt and dkk determine hair follicle spacing through a reaction-diffusion mechanism. Science, 314(5804):1447-1450, 2006.

[9] Kenneth Y. Wertheim and Tiina Roose. A mathematical model of lymphangiogenesis in a zebrafish embryo. Bulletin of Mathematical Biology, 79(4):693-737, Apr 2017. 
[10] Eshel Ben-Jacob, Ofer Schochet, Adam Tenenbaum, Inon Cohen, Andras Czirók, and Tamas Vicsek. Generic modelling of cooperative growth patterns in bacterial colonies. Nature, 368(6466):46-49, 1994.

[11] J. Lauterbach and H. H. Rotermund. Gas-phase coupling in the co oxidation reaction on polycrystalline platinum. Catalysis Letters, 27(1):27-32, 1994.

[12] BP Belousov. Periodicheski deistvuyushchaya reaktsia i ee mechanism. Sbornik Referatov po Radiatsionni Meditsine, 145147, 1958.

[13] Hans Meinhardt and Martin Klingler. A model for pattern formation on the shells of molluscs. Journal of Theoretical Biology, 126(1):63-89, 1987.

[14] M. A. Dahlem and S. C. Müller. Reaction-diffusion waves in neuronal tissue and the window of cortical excitability. Annalen der Physik, 13(7-8):442-449, 2020/01/14 2004.

[15] J. Christoph, M. Chebbok, C. Richter, J. Schröder-Schetelig, P. Bittihn, S. Stein, I. Uzelac, F. H. Fenton, G. Hasenfuß, R. F. Gilmour Jr., and S. Luther. Electromechanical vortex filaments during cardiac fibrillation. Nature, 555(7698):667-672, 2018.

[16] J.J. Tyson and J.D. Murray. Cyclic amp waves during aggregation of dictyostelium amoebae. Development, 106(3):421-426, 1989.

[17] Werner A. Mueller, Monika Hassel, and Maura Grealy. Development and Reproduction in Humans and Animal Model Species. Springer, 5. edition, 2015.

[18] O. Brefeld. Dictyostelium mucoroides ein neuer organismus aus der verwandschaft der myxomyceten. Abhandlungen der Senckenbergischen Naturforschenden Gesellschaft Frankfurt, 7:85-107, 1869.

[19] Kenneth B. Raper. Dictyostelium discoideum, a new species of slime mold from decaying forest leaves. J. Agr. Res., 50:135-147, 1935.

[20] Kenneth B. Raper. Growth and development of dictyostelium discoideum with different bacterial associates. J. Agr. Res., 55:289-316, 1937.

[21] Richard H. Kessin. Dictyostelium: Evolution, Cell Biology, and the Development of Multicellularity. Cambridge University Press, 2001.

[22] Rex L. Chisholm and Richard A. Firtel. Insights into morphogenesis from a simple developmental system. Nature Reviews Molecular Cell Biology, 5(7):531-541, 2004.

[23] Asha Rathi and Margaret Clarke. Expression of early developmental genes in dictyostelium discoideum is initiated during exponential growth by an autocrinedependent mechanism. Mechanisms of Development, 36(3):173 - 182, 1992. 
[24] Debra A Brock and Richard H Gomer. A cell-counting factor regulating structure size in dictyostelium. Genes \& development, 13(15):1960-1969, 1999.

[25] Cornelis J. Weijer. Spiral Waves of the Chemo-Attractant cAMP Organise Multicellular Development in the Social Amoebae Dictyostelium discoideum, pages 193207. Springer International Publishing, Cham, 2019.

[26] G. Gerisch. Stadienspezifische aggregationsmuster bei dictyostelium discoideum. Wilhelm Roux' Archiv für Entwicklungsmechanik der Organismen, 156(2):127-144, Jun 1965.

[27] Bakthier Vasiev, Florian Siegert, and Cornelis Weijer. Multiarmed spirals in excitable media. Phys. Rev. Lett., 78:2489-2492, Mar 1997.

[28] Mariko Katoh, Guokai Chen, Emily Roberge, Gad Shaulsky, and Adam Kuspa. Developmental commitment in dictyostelium discoideum. Eukaryotic Cell, 6(11):2038-2045, 2007.

[29] Hans R. Hohl and Jürg Jehli. The presence of cellulose microfibrils in the proteinaceous slime track of dictyostelium discoideum. Archiv für Mikrobiologie, 92(3):179187, Sep 1973.

[30] Jeffrey G. Williams, Karen T. Duffy, David P. Lane, Stuart J. McRobbie, Adrian J. Harwood, David Traynor, Robert R. Kay, and Keith A. Jermyn. Origins of the prestalk-prespore pattern in dictyostelium development. Cell, 59(6):1157 - 1163, 1989.

[31] Jens Rietdorf, Florian Siegert, and Cornelis J. Weijer. Analysis of optical density wave propagation and cell movement during mound formation indictyostelium discoideum. Developmental Biology, 177(2):427 - 438, 1996.

[32] Cornelis J Weijer. Dictyostelium morphogenesis. Current Opinion in Genetics 6 Development, 14(4):392-398, 2004.

[33] John Tyler Bonner, William Wight Clarke Jr, Charles Lea Neely Jr, and Mirian Kresses Slifkin. The orientation to light and the extermely sensitive orientation to temperature gradients in the slime mold dictyostelium discoideum. Journal of cellular and comparative physiology, 36(2):149-158, 1950.

[34] Kenneth B. Raper. Pseudoplasmodium formation and organization in dictyostelium discoideum. Journal of the Elisha Mitchell Scientific Society, 56(2):241$282,1940$.

[35] Paul R. Fisher, Elizabeth Smith, and Keith L. Williams. An extracellular chemical signal controlling phototactic behavior by d. discoideum slugs. Cell, 23(3):799 807, 1981. 
[36] F Siegert and C J Weijer. Three-dimensional scroll waves organize dictyostelium slugs. Proceedings of the National Academy of Sciences, 89(14):6433-6437, 1992.

[37] John Sternfeld. The anterior-like cells in dictyostelium are required for the elevation of the spores during culmination. Development Genes and Evolution, 208(9):487-494, Nov 1998.

[38] Hiroshi Abe, Masaaki Uchiyama, Yoshimasa Tanaka, and Hazime Saitô. Structure of discadenine, a spore germination inhibitor from the cellular slime mold, dictyostelium discoideum. Tetrahedron Letters, 17(42):3807 - 3810, 1976.

[39] John Tyler Bonner and Marya R. Dodd. Evidence for gas-induced orientation in the cellular slime molds. Developmental Biology, 5(2):344 - 361, 1962.

[40] Martin J. Huss. Dispersal of cellular slime molds by two soil invertebrates. Mycologia, 81(5):677-682, 1989.

[41] International Journal of Developmental Biology. The Dictyostelium model system, volume 63. UPV/EHU Press, 2019.

[42] PS Klein, TJ Sun, CL Saxe, AR Kimmel, RL Johnson, and PN Devreotes. A chemoattractant receptor controls development in dictyostelium discoideum. Science, 241(4872):1467-1472, 1988.

[43] CL 3rd Saxe, Gail T Ginsburg, John M Louis, Ronald Johnson, Peter N Devreotes, and Alan R Kimmel. Car2, a prestalk camp receptor required for normal tip formation and late development of dictyostelium discoideum. Genes $\&$ Development, 7(2):262-272, 1993.

[44] Ronald L Johnson, CL 3rd Saxe, Rachel Gollop, Alan R Kimmel, and Peter N Devreotes. Identification and targeted gene disruption of car3, a camp receptor subtype expressed during multicellular stages of dictyostelium development. Genes \& development, 7(2):273-282, 1993.

[45] John M Louis, Gail T Ginsburg, and Alan R Kimmel. The camp receptor car4 regulates axial patterning and cellular differentiation during late development of dictyostelium. Genes \& Development, 8(17):2086-2096, 1994.

[46] T Julia Sun and Peter N Devreotes. Gene targeting of the aggregation stage camp receptor car1 in dictyostelium. Genes \& Development, 5(4):572-582, 1991.

[47] J.Y. Kim, J.A. Borleis, and P.N. Devreotes. Switching of chemoattractant receptors programs development and morphogenesis in dictyostelium: Receptor subtypes activate common responses at different agonist concentrations. Developmental Biology, 197(1):117 - 128, 1998. 
[48] KJ Tomchik and PN Devreotes. Adenosine 3',5'-monophosphate waves in dictyostelium discoideum: a demonstration by isotope dilution-fluorography. Science, 212(4493):443-446, 1981.

[49] Sonya Bader, Arjan Kortholt, and Peter J. M. Van Haastert. Seven dictyostelium discoideum phosphodiesterases degrade three pools of camp and cgmp. Biochemical Journal, 402(1):153-161, 012007.

[50] K. Abe and K. Yanagisawa. A new class of rapidly developing mutants in dictyostelium discoideum: Implications for cyclic amp metabolism and cell differentiation. Developmental Biology, 95(1):200-210, 1983.

[51] Roberta K. Merkle, Kenneth K. Cooper, and Charles L. Rutherford. Localization and levels of cyclic amp during development of dictyostelium discoideum. Cell Differentiation, 14(4):257 - 266, 1984.

[52] A. F. M. Tariqul Islam, Margarethakay Scavello, Pouya Lotfi, Dustin Daniel, Pearce Haldeman, and Pascale G. Charest. Caffeine inhibits pi3k and mtorc2 in dictyostelium and differentially affects multiple other camp chemoattractant signaling effectors. Molecular and Cellular Biochemistry, 457(1):157-168, Jul 2019.

[53] A. Kumagai, M. Pupillo, R. Gundersen, R. Miake-Lye, P.N. Devreotes, and R.A. Firtel. Regulation and function of g $\alpha$ protein subunits in dictyostelium. Cell, 57(2):265 - 275, 1989.

[54] Joseph A Brzostowski, Cynthia Johnson, and Alan R Kimmel. G $\alpha$-mediated inhibition of developmental signal response. Current Biology, 12(14):1199 - 1208, 2002 .

[55] Joseph A Brzostowski, Carole A Parent, and Alan R Kimmel. A g $\alpha$-dependent pathway that antagonizes multiple chemoattractant responses that regulate directional cell movement. Genes \&6 development, 18(7):805-815, 2004.

[56] Peter N. Devreotes, Sayak Bhattacharya, Marc Edwards, Pablo A. Iglesias, Thomas Lampert, and Yuchuan Miao. Excitable signal transduction networks in directed cell migration. Annual Review of Cell and Developmental Biology, 33(1):103-125, 2017. PMID: 28793794.

[57] Christophe D. Reymond, Richard H. Gomer, Mona C. Mehdy, and Richard A. Firtel. Developmental regulation of a dictyostelium gene encoding a protein homologous to mammalian ras protein. Cell, 39(1):141 - 148, 1984.

[58] Stephen M. Robbins, Jeffrey G. Williams, George B. Spiegelman, and Gerald Weeks. Cloning and characterization of the dictyostelium discoideum rasg genomic sequences. Biochimica et Biophysica Acta (BBA) - Gene Structure and Expression, 1130(1):85 - 89, 1992. 
[59] Hyemin Mun and Taeck J. Jeon. Regulation of actin cytoskeleton by rap1 binding to racgef1. Molecules and Cells, 34(1):71-76, Jul 2012.

[60] J E Segall, A Kuspa, G Shaulsky, M Ecke, M Maeda, C Gaskins, R A Firtel, and W F Loomis. A map kinase necessary for receptor-mediated activation of adenylyl cyclase in dictyostelium. The Journal of Cell Biology, 128(3):405-413, 1995.

[61] M T Laub and W F Loomis. A molecular network that produces spontaneous oscillations in excitable cells of dictyostelium. Molecular biology of the cell, 9(12):35213532, 121998.

[62] Mineko Maeda, Sijie Lu, Gad Shaulsky, Yuji Miyazaki, Hidekazu Kuwayama, Yoshimasa Tanaka, Adam Kuspa, and William F. Loomis. Periodic signaling controlled by an oscillatory circuit that includes protein kinases erk2 and pka. Science, 304(5672):875-878, 2004.

[63] Satoru Funamoto, Ruedi Meili, Susan Lee, Lisa Parry, and Richard A. Firtel. Spatial and temporal regulation of 3-phosphoinositides by pi 3-kinase and pten mediates chemotaxis. Cell, 109(5):611 - 623, 2002.

[64] Parvin Bolourani, George B Spiegelman, and Gerald Weeks. Rap1 activation in response to camp occurs downstream of ras activation during dictyostelium aggregation. Journal of Biological Chemistry, 283(16):10232-10240, 2008.

[65] Satoru Funamoto, Kristina Milan, Ruedi Meili, and Richard A. Firtel. Role of phosphatidylinositol 3' kinase and a downstream pleckstrin homology domain-containing protein in controlling chemotaxis indictyostelium. The Journal of Cell Biology, 153(4):795-810, 2001.

[66] Frank I. Comer, Christopher K. Lippincott, Joseph J. Masbad, and Carole A. Parent. The pi3k-mediated activation of crac independently regulates adenylyl cyclase activation and chemotaxis. Current Biology, 15(2):134 - 139, 2005.

[67] Huaqing Cai, Satarupa Das, Yoichiro Kamimura, Yu Long, Carole A. Parent, and Peter N. Devreotes. Ras-mediated activation of the torc2-pkb pathway is critical for chemotaxis. The Journal of Cell Biology, 190(2):233-245, 2010.

[68] Ankita Khanna, Pouya Lotfi, Anita J. Chavan, Nieves M. Montaño, Parvin Bolourani, Gerald Weeks, Zhouxin Shen, Steven P. Briggs, Henderikus Pots, Peter J. M. Van Haastert, Arjan Kortholt, and Pascale G. Charest. The small gtpases ras and rap1 bind to and control torc2 activity. Scientific Reports, 6:25823 EP -, 052016.

[69] R Insall, A Kuspa, P J Lilly, G Shaulsky, L R Levin, W F Loomis, and P Devreotes. Crac, a cytosolic protein containing a pleckstrin homology domain, is required for receptor and g protein-mediated activation of adenylyl cyclase in dictyostelium. The Journal of Cell Biology, 126(6):1537-1545, 1994. 
[70] Geoffrey S. Pitt, Nina Milona, Jane Borleis, Kenneth C. Lin, Randall R. Reed, and Peter N. Devreotes. Structurally distinct and stage-specific adenylyl cyclase genes play different roles in dictyostelium development. Cell, 69(2):305 - 315, 1992.

[71] Hyun-Ji Kim, Wen-Tsan Chang, Marcel Meima, Julian D Gross, and Pauline Schaap. A novel adenylyl cyclase detected in rapidly developing mutants of dictyostelium. Journal of Biological Chemistry, 273(47):30859-30862, 1998.

[72] Saskia van Es, Kiran J Virdy, Geoffrey S Pitt, Marcel Meima, Todd W Sands, Peter N Devreotes, David A Cotter, and Pauline Schaap. Adenylyl cyclase g, an osmosensor controlling germination of dictyostelium spores. Journal of Biological Chemistry, 271(39):23623-23625, 1996.

[73] F. Soderbom, C. Anjard, N. Iranfar, D. Fuller, and W.F. Loomis. An adenylyl cyclase that functions during late development of dictyostelium. Development, 126(23):5463-5471, 1999.

[74] Margarethakay Scavello, Alexandra R. Petlick, Ramya Ramesh, Valery F. Thompson, Pouya Lotfi, and Pascale G. Charest. Protein kinase a regulates the ras, rap1 and torc 2 pathways in response to the chemoattractant camp in dictyostelium. Journal of Cell Science, 130(9):1545-1558, 2017.

[75] Kenneth M. Yamada and Michael Sixt. Mechanisms of 3d cell migration. Nature Reviews Molecular Cell Biology, 20(12):738-752, 2019.

[76] Dhiman S Pal, Xiaoguang Li, Tatsat Banerjee, Yuchuan Miao, and Peter N Devreotes. The excitable signal transduction networks: movers and shapers of eukaryotic cell migration. The International journal of developmental biology, 63(89-10):407-416, 2019.

[77] Yulia Artemenko, Lucas Axiotakis, Jane Borleis, Pablo A. Iglesias, and Peter N. Devreotes. Chemical and mechanical stimuli act on common signal transduction and cytoskeletal networks. Proceedings of the National Academy of Sciences, 113(47):E7500-E7509, 2016.

[78] Barbara Cortese, Ilaria Elena Palamà, Stefania D'Amone, and Giuseppe Gigli. Influence of electrotaxis on cell behaviour. Integrative Biology, 6(9):817-830, 07 2014.

[79] Taeck J. Jeon, Runchi Gao, Hyeseon Kim, Ara Lee, Pyeonghwa Jeon, Peter N. Devreotes, and Min Zhao. Cell migration directionality and speed are independently regulated by rasg and $\mathrm{g} \beta$ in dictyostelium cells in electrotaxis. Biology Open, 8(7), 2019.

[80] Donat-P. Häder and Kenneth L. Poff. Light-induced accumulations of dictyostelium discoideum amoebae. Photochemistry and Photobiology, 29(6):1157$1162,2020 / 01 / 281979$. 
[81] Emmanuel Décavé, Didier Rieu, Jérémie Dalous, Sébastien Fache, Yves Bréchet, Bertrand Fourcade, Michel Satre, and Franz Bruckert. Shear flow-induced motility of dictyostelium discoideum cells on solid substrate. Journal of Cell Science, 116(21):4331-4343, 2003.

[82] John ME Nichols, Douwe Veltman, and Robert R Kay. Chemotaxis of a model organism: progress with dictyostelium. Current Opinion in Cell Biology, 36:7-12, 2015.

[83] Yuchuan Miao, Sayak Bhattacharya, Marc Edwards, Huaqing Cai, Takanari Inoue, Pablo A. Iglesias, and Peter N. Devreotes. Altering the threshold of an excitable signal transduction network changes cell migratory modes. Nature Cell Biology, 19(4):329-340, 2017.

[84] Gareth Bloomfield, David Traynor, Sophia P Sander, Douwe M Veltman, Justin A Pachebat, Robert R Kay, and W James Nelson. Neurofibromin controls macropinocytosis and phagocytosis in dictyostelium. eLife, 4:e04940, 2015.

[85] Nicholas P. Barry and Mark S. Bretscher. Dictyostelium amoebae and neutrophils can swim. Proceedings of the National Academy of Sciences, 107(25):11376, 06 2010 .

[86] Jonathan D Howe, Nicholas P Barry, and Mark S Bretscher. How do amoebae swim and crawl? PloS one, 8(9):e74382-e74382, 092013.

[87] J.T. Bonner, D.S. Barkley, E.M. Hall, T.M. Konijn, J.W. Mason, G. O’Keefe, and P.B. Wolfe. Acrasin, acrasinase, and the sensitivity to acrasin in dictyostelium discoideum. Developmental Biology, 20(1):72 - 87, 1969.

[88] Peter J.M. Van Haastert, René J.W. De Wit, and Theo M. Konijn. Antagonists of chemoattractants reveal separate receptors for camp, folk acid and pterin in dictyostelium. Experimental Cell Research, 140(2):453 - 456, 1982.

[89] M C Dinauer, S A MacKay, and P N Devreotes. Cyclic 3',5'-amp relay in dictyostelium discoideum iii. the relationship of camp synthesis and secretion during the camp signaling response. The Journal of Cell Biology, 86(2):537-544, 1980.

[90] Paul W. Kriebel, Valarie A. Barr, and Carole A. Parent. Adenylyl cyclase localization regulates streaming during chemotaxis. Cell, 112(4):549-560, 2003.

[91] Paul W. Kriebel, Ritankar Majumdar, Lisa M. Jenkins, Hiroshi Senoo, Weiye Wang, Sonia Ammu, Song Chen, Kedar Narayan, Miho Iijima, and Carole A. Parent. Extracellular vesicles direct migration by synthesizing and releasing chemotactic signals. The Journal of Cell Biology, 217(8):2891-2910, 062018.

[92] A Theibert and P N Devreotes. Cyclic 3', 5'-amp relay in dictyostelium discoideum: adaptation is independent of activation of adenylate cyclase. The Journal of Cell Biology, 97(1):173-177, 1983. 
[93] Loling Song, Sharvari M. Nadkarni, Hendrik U. Bödeker, Carsten Beta, Albert Bae, Carl Franck, Wouter-Jan Rappel, William F. Loomis, and Eberhard Bodenschatz. Dictyostelium discoideum chemotaxis: Threshold for directed motion. European Journal of Cell Biology, 85(9):981-989, 2006.

[94] Roxanne A Vaughan and Peter N Devreotes. Ligand-induced phosphorylation of the camp receptor from dictyostelium discoideum. Journal of Biological Chemistry, 263(28):14538-14543, 1988.

[95] M Dinauer, TL Steck, and P Devreotes. Cyclic 3', 5'-amp relay dictyostelium discoideum. v. adaptation of the camp signaling response during camp stimulation. The Journal of Cell Biology, 86(2):554-561, 1980.

[96] M Dinauer, TL Steck, and P Devreotes. Cyclic 3', 5'-amp relay in dictyostelium discoideum. iv. recovery of the camp signaling response after adaptation to camp. The Journal of Cell Biology, 86(2):545-553, 1980.

[97] Ying-Ying Chang. Cyclic 3',5'-adenosine monophosphate phosphodiesterase produced by the slime mold dictyostelium discoideum. Science, 161(3836):57-59, 1968.

[98] Gene L. Garcia, Erin C. Rericha, Christopher D. Heger, Paul K. Goldsmith, and Carole A. Parent. The group migration of dictyostelium cells is regulated by extracellular chemoattractant degradation. Molecular Biology of the Cell, 20(14):32953304, 2009. PMID: 19477920.

[99] G Gerisch, D Malchow, V Riedel, E Müller, and M Every. Cyclic amp phosphodiesterase and its inhibitor in slime mould development. Nature New Biology, 235(55):90-92, 1972.

[100] T. M. Konijn, D. S. Barkley, Y. Y. Chang, and J. T. Bonner. Cyclic amp: A naturally occurring acrasin in the cellular slime molds. The American Naturalist, 102(925):225-233, 1968.

[101] Fernanda Alcantara and Marilyn Monk. Signal propagation during aggregation in the slime mould dictyostelium discoideum. Microbiology, 85(2):321-334, 1974.

[102] J M Mato, A Losada, V Nanjundiah, and T M Konijn. Signal input for a chemotactic response in the cellular slime mold dictyostelium discoideum. Proceedings of the National Academy of Sciences, 72(12):4991-4993, 1975.

[103] Keita Kamino, Yohei Kondo, Akihiko Nakajima, Mai Honda-Kitahara, Kunihiko Kaneko, and Satoshi Sawai. Fold-change detection and scale invariance of cell-cell signaling in social amoeba. Proceedings of the National Academy of Sciences, 114(21):E4149-E4157, 2017. 
[104] Monica Skoge, Haicen Yue, Michael Erickstad, Albert Bae, Herbert Levine, Alex Groisman, William F. Loomis, and Wouter-Jan Rappel. Cellular memory in eukaryotic chemotaxis. Proceedings of the National Academy of Sciences, 111(40):14448-14453, 2014.

[105] Herbert Levine and William Reynolds. Streaming instability of aggregating slime mold amoebae. Phys. Rev. Lett., 66:2400-2403, May 1991.

[106] R P Futrelle, J Traut, and W G McKee. Cell behavior in dictyostelium discoideum: preaggregation response to localized cyclic amp pulses. The Journal of Cell Biology, 92(3):807-821, 1982.

[107] Edith Schäfer, Marco Tarantola, Elena Polo, Christian Westendorf, Noriko Oikawa, Eberhard Bodenschatz, Burkhard Geil, and Andreas Janshoff. Chemotaxis of dictyostelium discoideum: Collective oscillation of cellular contacts. PLOS ONE, 8(1):e54172-, 012013.

[108] Florian Siegert and Cornelis J. Weijer. Analysis of optical density wave propagation and cell movement in the cellular slime mould dictyostelium discoideum. Physica D: Nonlinear Phenomena, 49(1):224 - 232, 1991.

[109] Kyoung J. Lee, Edward C. Cox, and Raymond E. Goldstein. Competing patterns of signaling activity in dictyostelium discoideum. Phys. Rev. Lett., 76:1174-1177, Feb 1996.

[110] Florian Siegert and Cornelis Weijer. Digital image processing of optical density wave propagation in dictyostelium discoideum and analysis of the effects of caffeine and ammonia. Journal of Cell Science, 93(2):325-335, 1989.

[111] J.D. Gross, M.J. Peacey, and D.J. Trevan. Signal emission and signal propagation during early aggregation in dictyostelium discoideum. Journal of Cell Science, 22(3):645-656, 1976.

[112] A.J. Durston. Pacemaker activity during aggregation in dictyostelium discoideum. Developmental Biology, 37(2):225 - 235, 1974.

[113] Thomas Gregor, Koichi Fujimoto, Noritaka Masaki, and Satoshi Sawai. The onset of collective behavior in social amoebae. Science, 328(5981):1021-1025, 2010.

[114] Peter N. Devreotes, Michael J. Potel, and Stephen A. MacKay. Quantitative analysis of cyclic amp waves mediating aggregation in dictyostelium discoideum. Developmental Biology, 96(2):405 - 415, 1983.

[115] Juliet C. Coates and Adrian J. Harwood. Cell-cell adhesion and signal transduction during dictyostelium development. Journal of Cell Science, 114(24):4349-4358, 2001. 
[116] Günther Gerisch. Chapter 6 cell aggregation and differentiation in dictyostelium. volume 3 of Current Topics in Developmental Biology, pages 157 - 197. Academic Press, 1968.

[117] Geoffrey S Pitt, Raymond Brandt, Kenneth C Lin, Peter N Devreotes, and Pauline Schaap. Extracellular camp is sufficient to restore developmental gene expression and morphogenesis in dictyostelium cells lacking the aggregation adenylyl cyclase (aca). Genes 65 development, 7(11):2172-2180, 1993.

[118] Michael Brenner and Stephen D. Thoms. Caffeine blocks activation of cyclic amp synthesis in dictyostelium discoideum. Developmental Biology, 101(1):136 - 146, 1984.

[119] Steinbock Oliver and Müller Stefan C. Spatial attractors in aggregation patterns of dictyostelium discoideum. Zeitschrift für Naturforschung C, 50:275, 2019-1101T16:44:47.316+01:00 1995.

[120] Edith Schäfer, Christian Westendorf, Eberhard Bodenschatz, Carsten Beta, Burkhard Geil, and Andreas Janshoff. Shape oscillations of dictyostelium discoideum cells on ultramicroelectrodes monitored by impedance analysis. Small, 7(6):723-726, 2020/01/30 2011.

[121] Pundrik Jaiswal, Thierry Soldati, Sascha Thewes, and Ramamurthy Baskar. Regulation of aggregate size and pattern by adenosine and caffeine in cellular slime molds. BMC Developmental Biology, 12(1):5, 2012.

[122] Robert R Kay, Thomas D Williams, James D Manton, David Traynor, and Peggy Paschke. Living on soup: macropinocytic feeding in amoebae. International Journal of Developmental Biology, 63(8-9-10):473-483, 2019.

[123] U. Hacker, R. Albrecht, and M. Maniak. Fluid-phase uptake by macropinocytosis in dictyostelium. Journal of Cell Science, 110(2):105-112, 1997.

[124] R. Sussman and M. Sussman. Cultivation of dictyostelium discoideum in axenic medium. Biochemical and Biophysical Research Communications, 29(1):53 - 55, 1967.

[125] D J Watts and J M Ashworth. Growth of myxameobae of the cellular slime mould dictyostelium discoideum in axenic culture. The Biochemical journal, 119(2):171174, 091970.

[126] Gregory W. Erdos, Kenneth B. Raper, and Linda K. Vogen. Mating types and macrocyst formation in dictyostelium discoideum. Proceedings of the National Academy of Sciences, 70(6):1828-1830, 1973.

[127] W.F. Loomis. Sensitivity of dictyostelium discoideum to nucleic acid analogues. Experimental Cell Research, 64(2):484 - 486, 1971. 
[128] A. N. Zaikin and A. M. Zhabotinsky. Concentration wave propagation in twodimensional liquid-phase self-oscillating system. Nature, 225(5232):535-537, 1970.

[129] A. T. Winfree. The prehistory of the belousov-zhabotinsky oscillator. Journal of Chemical Education, 61(8):661, 1984.

[130] Alexander A Pechenkin. Understanding of the history of the belousov-zhabotinsky reaction. Studia Philosophica IV, 40:106-130, 2004.

[131] Richard J. Field, Endre Koros, and Richard M. Noyes. Oscillations in chemical systems. ii. thorough analysis of temporal oscillation in the bromate-cerium-malonic acid system. Journal of the American Chemical Society, 94(25):8649-8664, 12 1972.

[132] VA Vavilin and Ao N Zaikin. Effect of solution mixing on a rate of the autocatalytic reaction. Kinet. catal, 12:309-313, 1971.

[133] Nadeem B. Ganaie and G. M. Peerzada. Catalyst, Co-ion and the media effect on the oscillatory behavior of resorcinol in the $\mathrm{BZ}$ reaction. Journal of the Brazilian Chemical Society, 20:1262 - 1267, 002009.

[134] John J. Tyson. Some further studies of nonlinear oscillations in chemical systems. The Journal of Chemical Physics, 58(9):3919-3930, 1973.

[135] J. A. C. Gallas. Periodic oscillations of the forced brusselator. Modern Physics Letters B, 29(35n36):1530018, 2015.

[136] Peter Kovesi. Good colour maps: How to design them, 2015.

[137] Michael Cross and Henry Greenside. Pattern Formation and Dynamics in Nonequilibrium Systems. Cambridge university press, 2009.

[138] Lingfa Yang, Milos Dolnik, Anatol M. Zhabotinsky, and Irving R. Epstein. Pattern formation arising from interactions between turing and wave instabilities. The Journal of Chemical Physics, 117(15):7259-7265, 2002.

[139] John J. Tyson and Paul C. Fife. Target patterns in a realistic model of the belousov-zhabotinskii reaction. The Journal of Chemical Physics, 73(5):22242237, 1980 .

[140] A. L. Hodgkin and A. F. Huxley. The components of membrane conductance in the giant axon of loligo. The Journal of Physiology, 116(4):473-496, 1952.

[141] A. L. Hodgkin and A. F. Huxley. A quantitative description of membrane current and its application to conduction and excitation in nerve. The Journal of Physiology, 117(4):500-544, 1952.

[142] Richard FitzHugh. Mathematical models of threshold phenomena in the nerve membrane. The bulletin of mathematical biophysics, 17(4):257-278, Dec 1955. 
[143] Richard FitzHugh. Impulses and physiological states in theoretical models of nerve membrane. Biophysical Journal, 1(6):445 - 466, 1961.

[144] J. Nagumo, S. Arimoto, and S. Yoshizawa. An active pulse transmission line simulating nerve axon. Proceedings of the IRE, 50(10):2061-2070, Oct 1962.

[145] George Potts. Zur physiologie des dictyostelium mucoroides. Flora, 91:281-347, 1902.

[146] Edgar William Olive. Monograph of the acrasieae. Boston Society of Natural History, 1902.

[147] EH Runyon. Aggregation of separate cells of dictyostelium to form a multicellular body. Collecting Net, 17:88, 1942.

[148] John Tyler Bonner and L. J. Savage. Evidence for the formation of cell aggregates by chemotaxis in the development of the slime mold dictyostelium discoideum. Journal of Experimental Zoology, 106(1):1-26, 1947.

[149] Morrel H. Cohen and Anthony Robertson. Wave propagation in the early stages of aggregation of cellular slime molds. Journal of Theoretical Biology, 31(1):101 118, 1971.

[150] Evelyn F. Keller and Lee A. Segel. Initiation of slime mold aggregation viewed as an instability. Journal of Theoretical Biology, 26(3):399 - 415, 1970.

[151] P.S. Hagan and M.S. Cohen. Diffusion-induced morphogenesis in the development of dictyostelium. Journal of Theoretical Biology, 93(4):881 - 908, 1981.

[152] Peter N Devreotes and JA Sherring. Kinetics and concentration dependence of reversible camp-induced modification of the surface camp receptor in dictyostelium. Journal of Biological Chemistry, 260(10):6378-6384, 1985.

[153] W. Roos and G. Gerisch. Receptor-mediated adenylate cyclase activation in dictyostelium discoideum. FEBS Letters, 68(2):170-172, 1976.

[154] G Gerisch and D Malchow. Cyclic amp receptors and the control of cell aggregation in dictyostelium. Advances in cyclic nucleotide research, 7:49, 1976.

[155] Claudette Klein. A slowly dissociating form of the cell surface cyclic adenosine 3': 5'-monophosphate receptor of dictyostelium discoideum. Journal of Biological Chemistry, 254(24):12573-12578, 1979.

[156] Jean-Louis Martiel and Albert Goldbeter. A model based on receptor desensitization for cyclic amp signaling in dictyostelium cells. Biophysical Journal, 52(5):807 $-828,1987$. 
[157] Torsten Eckstein, Estefania Vidal-Henriquez, Albert Bae, Vladimir Zykov, Eberhard Bodenschatz, and Azam Gholami. Influence of fast advective flows on pattern formation of dictyostelium discoideum. PLOS ONE, 13(3):e0194859-, 032018.

[158] John J. Tyson, Kevin A. Alexander, V.S. Manoranjan, and J.D. Murray. Spiral waves of cyclic amp in a model of slime mold aggregation. Physica D: Nonlinear Phenomena, 34(1):193 - 207, 1989.

[159] José Halloy, Jacques Lauzeral, and Albert Goldbeter. Modeling oscillations and waves of camp in dictyostelium discoideum cells. Biophysical Chemistry, 72(1):9 19, 1998.

[160] A Gholami, O Steinbock, V Zykov, and E Bodenschatz. Flow-driven instabilities during pattern formation of dictyostelium discoideum. New Journal of Physics, 17(6):063007, jun 2015.

[161] Arkady B. Rovinsky and Michael Menzinger. Chemical instability induced by a differential flow. Phys. Rev. Lett., 69:1193-1196, Aug 1992.

[162] Arkady B. Rovinsky and Michael Menzinger. Self-organization induced by the differential flow of activator and inhibitor. Phys. Rev. Lett., 70:778-781, Feb 1993.

[163] Robert A. Van Gorder, Hyunyeon Kim, and Andrew L. Krause. Diffusive instabilities and spatial patterning from the coupling of reaction-diffusion processes with stokes flow in complex domains. Journal of Fluid Mechanics, 877:759-823, 2019.

[164] Evelyn Fox Keller. The force of the pacemaker concept in theories of aggregation in cellular slime mold. Perspectives in biology and medicine, 26(4):515-521, 1983.

[165] B.M. Shaffer. The acrasina. volume 2 of Advances in Morphogenesis, pages 109 182. Elsevier, 1962.

[166] Yusaku Ohta, Toshiaki Furuta, Takeharu Nagai, and Kazuki Horikawa. Red fluorescent camp indicator with increased affinity and expanded dynamic range. Scientific Reports, 8(1):1866, 2018.

[167] Hsin-Fang Hsu, Eberhard Bodenschatz, Christian Westendorf, Azam Gholami, Alain Pumir, Marco Tarantola, and Carsten Beta. Variability and order in cytoskeletal dynamics of motile amoeboid cells. Phys. Rev. Lett., 119:148101, Oct 2017.

[168] Jacques Lauzeral, José Halloy, and Albert Goldbeter. Desynchronization of cells on the developmental path triggers the formation of spiral waves of camp during dictyostelium aggregation. Proceedings of the National Academy of Sciences, 94(17):9153-9158, 1997.

[169] R. Dillon, P. K. Maini, and H. G. Othmer. Pattern formation in generalized turing systems. Journal of Mathematical Biology, 32(4):345-393, Apr 1994. 
[170] P.K. Maini and M.R. Myerscough. Boundary-driven instability. Applied Mathematics Letters, 10(1):1 - 4, 1997.

[171] Jonathan A Sherratt. Periodic travelling wave selection by dirichlet boundary conditions in oscillatory reaction-diffusion systems. SIAM Journal on Applied Mathematics, 63(5):1520-1538, 2003.

[172] Estefania Vidal-Henriquez, Vladimir Zykov, Eberhard Bodenschatz, and Azam Gholami. Convective instability and boundary driven oscillations in a reactiondiffusion-advection model. Chaos: An Interdisciplinary Journal of Nonlinear Science, 27(10):103110, 2017.

[173] Estefania Vidal-Henriquez and Azam Gholami. Spontaneous center formation in dictyostelium discoideum. Scientific Reports, 9(1):3935, 2019.

[174] Mercedes Barchilon and Lee A. Segel. Adaptation, oscillations and relay in a model for camp secretion in cellular slime molds. Journal of Theoretical Biology, 133(4):437 - 446, 1988.

[175] David A. Kessler and Herbert Levine. Pattern formation in dictyostelium via the dynamics of cooperative biological entities. Phys. Rev. E, 48:4801-4804, Dec 1993.

[176] Kaumudi H Prabhakara, Azam Gholami, Vladimir S Zykov, and Eberhard Bodenschatz. Effects of developmental variability on the dynamics and self-organization of cell populations. New Journal of Physics, 19(11):113024, nov 2017.

[177] Kaumudi H Prabhakara, Albert J Bae, and Eberhard Bodenschatz. Cooperation of the haves and the have-nots. bioRxiv, 2017.

[178] J Franke and R Kessin. A defined minimal medium for axenic strains of dictyostelium discoideum. Proceedings of the National Academy of Sciences of the United States of America, 74(5):2157-2161, 051977.

[179] Arthur D. Edelstein, Mark A. Tsuchida, Nenad Amodaj, Henry Pinkard, Ronald D. Vale, and Nico Stuurman. Advanced methods of microscope control using $\mu$ manager software. Journal of biological methods, 1(2), 2014.

[180] George M. Whitesides, Emanuele Ostuni, Shuichi Takayama, Xingyu Jiang, and Donald E. Ingber. Soft lithography in biology and biochemistry. Annual Review of Biomedical Engineering, 3(1):335-373, 2001. PMID: 11447067.

[181] David A Egolf, Ilarion V Melnikov, and Eberhard Bodenschatz. Importance of local pattern properties in spiral defect chaos. Physical Review Letters, 80(15):3228, 1998.

[182] Julius S Bendat and Allan G Piersol. Random data: analysis and measurement procedures, volume 729. John Wiley \& Sons, 2011. 
[183] Florian Siegert and Cornelis J. Weijer. Spiral and concentric waves organize multicellular dictyostelium mounds. Current Biology, 5(8):937 - 943, 1995.

[184] Kyoung J. Lee, Edward C. Cox, and Raymond E. Goldstein. Competing patterns of signaling activity in dictyostelium discoideum. Phys. Rev. Lett., 76:1174-1177, Feb 1996.

[185] Kyoung J. Lee, Raymond E. Goldstein, and Edward C. Cox. Resetting wave forms in dictyostelium territories. Phys. Rev. Lett., 87:068101, Jul 2001.

[186] Satoshi Sawai, Xiao-Juan Guan, Adam Kuspa, and Edward C. Cox. Highthroughput analysis of spatio-temporal dynamics in dictyostelium. Genome Biology, 8(7):R144, 2007.

[187] Elmar Schrüfer. Signalverarbeitung - Numerische Verarbeitung digitaler Signale. Carl Hanser Verlag, 1992.

[188] M.P. Mosley. Subsurface flow velocities through selected forest soils, south island, new zealand. Journal of Hydrology, 55(1):65 - 92, 1982.

[189] A. Gholami, O. Steinbock, V. Zykov, and E. Bodenschatz. Flow-driven waves and phase-locked self-organization in quasi-one-dimensional colonies of dictyostelium discoideum. Phys. Rev. Lett., 114:018103, Jan 2015.

[190] A Gholami, V Zykov, O Steinbock, and E Bodenschatz. Flow-driven twodimensional waves in colonies of dictyostelium discoideum. New Journal of Physics, 17(9):093040, sep 2015.

[191] Elena A. Ermakova, Emmanuil E. Shnol, Mikhail A. Panteleev, Andrey A. Butylin, Vitaly Volpert, and Fazoil I. Ataullakhanov. On propagation of excitation waves in moving media: The fitzhugh-nagumo model. PLOS ONE, 4(2):e4454-, 022009.

[192] Lenka Sebestikova, Elena Slamova, and Hana Sevcikova. Control of wave propagation in a biological excitable medium by an external electric field. Biophysical Chemistry, 113(3):269-274, 2005.

[193] Jiří Lindner, Hana Ševčíková, and Miloš Marek. Influence of an external electric field on camp wave patterns in aggregating dictyostelium discoideum. Physical Review E, 63(4):041904-, 032001.

[194] Isaac Levy, Anelia Horvath, Monalisa Azevedo, Rodrigo Bertollo de Alexandre, and Constantine A Stratakis. Phosphodiesterase function and endocrine cells: links to human disease and roles in tumor development and treatment. Current opinion in pharmacology, 11(6):689-697, 122011.

[195] Malee Sutthiopad, Jiraporn Luengviriya, Porramain Porjai, Metinee Phantu, Jarin Kanchanawarin, Stefan C. Müller, and Chaiya Luengviriya. Propagation of spiral 
waves pinned to circular and rectangular obstacles. Phys. Rev. E, 91:052912, May 2015 .

[196] Zhihui Zhang and Oliver Steinbock. Local heterogeneities in cardiac systems suppress turbulence by generating multi-armed rotors. New Journal of Physics, 18(5):053018, may 2016.

[197] Michael W. Toepke and David J. Beebe. Pdms absorption of small molecules and consequences in microfluidic applications. Lab on a Chip, 6(12):1484-1486, 2006.

[198] O. Steinbock and S. C. Müller. Light-controlled anchoring of meandering spiral waves. Phys. Rev. E, 47:1506-1509, Mar 1993.

[199] Malee Sutthiopad, Jiraporn Luengviriya, Porramain Porjai, Boosayarat Tomapatanaget, Stefan C. Müller, and Chaiya Luengviriya. Unpinning of spiral waves by electrical forcing in excitable chemical media. Phys. Rev. E, 89:052902, May 2014.

[200] Vladimir S. Zykov and Eberhard Bodenschatz. Wave propagation in inhomogeneous excitable media. Annual Review of Condensed Matter Physics, 9(1):435-461, 2020/01/07 2018.

[201] Hana Ševčíková, Jitka Čejková, Lenka Krausová, Michal Přibyl, František Štěpánek, and Miloš Marek. A new traveling wave phenomenon of dictyostelium in the presence of camp. Physica D: Nonlinear Phenomena, 239(11):879-888, 2010.

[202] NG Holmer, A Johnsson, et al. Effects of ultrasonic irradiation upon amoeba proteus. Zeitschrift für Naturforschung C, 28(9-10):607-609, 1973.

[203] Andrew Fleischman, Christopher Vecchio, Youhan Sunny, Christopher R. Bawiec, Peter A. Lewin, J. Yasha Kresh, and Andrew R. Kohut. Ultrasound-induced modulation of cardiac rhythm in neonatal rat ventricular cardiomyocytes. Journal of Applied Physiology, 118(11):1423-1428, 2020/01/07 2015.

[204] D. A. Hughes, C. J. Weijer, Yongqiang Qiu, C. Demore, and S. Cochran. Investigating the motility of dictyostelium discodeum using high frequency ultrasound as a method of manipulation. In 2012 IEEE International Ultrasonics Symposium, pages 2002-2005, 2012.

[205] Linnan Tang, Jonathan Franca-Koh, Yuan Xiong, Mei-Yu Chen, Yu Long, Ross M Bickford, David A Knecht, Pablo A Iglesias, and Peter N Devreotes. tsunami, the dictyostelium homolog of the fused kinase, is required for polarization and chemotaxis. Genes \& development, 22(16):2278-2290, 2008. 



\section{Acknowledgments}

I thank Dr. Azam Gholami for guiding me during my $\mathrm{PhD}$ and teaching me how to conduct research in a self-reliant manner. The work presented in this thesis would not have been possible without her pointing me towards interesting avenues of research. Dr. Gholamis approach to supervision was centered on allowing independent research while also giving advice and helping whenever needed. During my time in her group I felt that she was trying her best to help me succeed in my PhD studies.

My thanks to Prof. Bodenschatz for allowing me to join his group, which has a unique mixture of theoretical and experimental approaches.

I thank the DFG for funding this project - project number GH 184/1-1.

In regards to my experimental work, I thank Maren Stellar Müller for supplying the cell culture I worked with and answering countless questions on lab work. Similarly, I want to thank Katharina Gunkel for having an open ear to any question I came up with and giving many pieces of advice. In general, I thank the lab team who helped me in many ways during my stay.

Considering scientific discussions and advice, I thank Dr. Marco Tarantola, with whom I had many discussions on D. discoideum cells, Dr. Vladimir Zykov, who helped me understand excitable media, Holger Nobach whom I asked a number of questions on signal processing and Albert Bae who was an invaluable help in both the experimental and the analysis part of this work.

My thanks also to the team from the mechanical and the electronic workshop for helping me realize my experimental setups.

I want to thank the team of the PBCS office for help and advice on all matters about the bureaucracy of this PhD.

Finally, I thank my family for motivating and supporting me. Thank you Ina - for help and advice all throughout my education and today. Thanks to Barbara for believing in me, cheering me up and being yourself. Thanks to Tanja for being the best sister I could ever hope for. 
